\title{
METALLICITY DISTRIBUTION FUNCTIONS, RADIAL VELOCITIES, AND ALPHA ELEMENT ABUNDANCES IN THREE OFF-AXIS BULGE FIELDS
}

\author{
Christian I. Johnson ${ }^{1,6,7,8}$, R. Michael Rich ${ }^{1}$, Chiaki Kobayashi ${ }^{2}$, Andrea \\ Kunder $^{3}$, Catherine A. Pilachowski ${ }^{4}$, Andreas Koch ${ }^{5}$, and Roberto de \\ Propris $^{3}$
}

\begin{abstract}
We present radial velocities and chemical abundance ratios of $[\mathrm{Fe} / \mathrm{H}],[\mathrm{O} / \mathrm{Fe}]$, $[\mathrm{Si} / \mathrm{Fe}]$, and $[\mathrm{Ca} / \mathrm{Fe}]$ for 264 red giant branch $(\mathrm{RGB})$ stars in three Galactic bulge off-axis fields located near $(1, b)=(-5.5,-7),(-4,-9)$, and $(+8.5,+9)$. The results are based on equivalent width and spectrum synthesis analyses of moderate resolution $(\mathrm{R} \approx 18,000)$, high signal-to-noise ratio $\left(\mathrm{S} / \mathrm{N} \sim 75-300 \mathrm{pixel}^{-1}\right)$ spectra obtained with the Hydra spectrographs on the Blanco $4 \mathrm{~m}$ and WIYN $3.5 \mathrm{~m}$ telescopes. The targets were selected from the blue side of the giant branch to avoid cool stars that would be strongly affected by $\mathrm{CN}$ and $\mathrm{TiO}$; however, a comparison of the color-metallicity distribution in literature samples suggests our selection of bluer targets should not present a significant bias against metalrich stars. We find a full range in metallicity that spans $[\mathrm{Fe} / \mathrm{H}] \approx-1.5$ to +0.5 , and that, in accordance with the previously observed minor-axis vertical metallicity gradient, the median $[\mathrm{Fe} / \mathrm{H}]$ also declines with increasing Galactic latitude in off-axis fields. The off-axis vertical $[\mathrm{Fe} / \mathrm{H}]$ gradient in the southern bulge is estimated to be $\sim 0.4$ dex kpc$^{-1}$; however, comparison with the minor-axis data suggests a strong radial gradient does not exist. The $(+8.5,+9)$ field exhibits a higher than expected metallicity, with a median $[\mathrm{Fe} / \mathrm{H}]=-0.23$, that might be related to a stronger presence of the $\mathrm{X}$-shaped bulge structure along that line- ofsight. This could also be the cause of an anomalous increase in the median radial velocity for intermediate metallicity stars in the $(+8.5,+9)$ field. However, the overall radial velocity and dispersion for each field are in good agreement with recent surveys and bulge models. All fields exhibit an identical, strong decrease in velocity dispersion with increasing metallicity that is consistent with observations in similar minor-axis outer bulge fields. Additionally, the [O/Fe], [Si/Fe], and $[\mathrm{Ca} / \mathrm{Fe}]$ versus $[\mathrm{Fe} / \mathrm{H}]$ trends are identical among our three fields, and are in good agreement with past bulge studies. We find that stars with $[\mathrm{Fe} / \mathrm{H}] \lesssim-$ 0.5 are $\alpha$-enhanced, and that the $[\alpha / \mathrm{Fe}]$ ratios decline at higher metallicity. At $[\mathrm{Fe} / \mathrm{H}] \lesssim 0$, the $\alpha$-element trends are indistinguishable from the halo and thick disk, and the variations in the behavior of individual $\alpha$-elements are consistent with production in massive stars and a rapid buloe formation timescale.
\end{abstract}




\section{INTRODUCTION}

Although the minor-axis of the Galactic bulge has been the subject of extensive high resolution spectroscopic study (e.g., Zoccali et al. 2008), there has been relatively little chemical characterization of stars in off-axis bulge fields. Iron and $\alpha^{-}$ element abundances are reported by Gonzalez et al. (2011) in a field near the globular cluster NGC $6553(\mathrm{l}, \mathrm{b})=(+5.25,--$ 3 ), but that is the only off-axis field for

\footnotetext{
${ }^{1}$ Department of Physics and Astronomy, UCLA, 430 Portola Plaza, Box 951547, Los Angeles, CA 90095-1547, USA; cijohnson@astro.ucla.edu; rmr@astro.ucla.edu

${ }^{2}$ Centre for Astrophysics Research, University of Hertfordshire, Hatfield AL10 9AB, UK; c.kobayashi@herts.ac.uk

${ }^{3}$ Cerro Tololo Inter-American Observatory, Casilla 603, La Serena, Chile; akunder@ctio.noao.edu

${ }^{4}$ Department of Astronomy, Indiana University, Swain West 319, 727 East Third Street, Bloomington, IN 47405-7105, USA; catyp@astro.indiana.edu

${ }^{5}$ Zentrum für Astronomie der Universität Heidelberg, Landessternwarte, Königstuhl 12, Heidelberg, Germany; akoch@lsw.uni-heidelberg.de

${ }^{6}$ Visiting astronomer, Cerro Tololo InterAmerican Observatory, National Optical Astronomy Observatory, which are operated by the Association of Universities for Research in Astronomy, under contract with the National Science Foundation.

${ }^{7}$ Visiting Astronomer, Kitt Peak National Observatory, National Optical Astronomy Observatories, which is operated by the Association of Universities for Research in Astronomy, Inc. (AURA) under cooperative agreement with the National Science Foundation. The WIYN Observatory is a joint facility of the University of WisconsinMadison, Indiana University, Yale University, and the National Optical Astronomy Observatory.

${ }^{8}$ National Science Foundation Astronomy and Astrophysics Postdoctoral Fellow
}

which significant $(\mathrm{N} \gtrsim 100$ stars $)$ chemical abundance data are presently available1. Overall, the radial variation in $[\mathrm{Fe} / \mathrm{H}]^{2}$ and $[\alpha / \mathrm{Fe}]$ along the bulge major-axis and offaxis is poorly constrained.

It is also clear that the bulge is spatially complex, and a deep understanding will require a full three-dimensional sampling of the structure. McWilliam \& Zoccali (2010) found red clump giants to be spatially distributed in an $\mathrm{X}$-shaped structure that spans the inner $\sim 8^{\circ}$, and was confirmed in a density analysis by Saito et al. (2011). The metal-rich subset of the bulge appears to be predominant in the $\mathrm{X}$ shaped structure (Ness et al. 2012; Uttenthaler et al. 2012; but see also de Propris et al. 2011).

Connected with these issues is the question of whether there is kinematic substructure in the bulge, and whether the population is comprised of multiple components. Some evidence suggests the bulge may host two dominant populations (Babusiaux et al. 2010; Bensby et al. 2011; Hill et al. 2011; Uttenthaler et al. 2012). At the most basic level, such a bimodality might be expressed in the bar and the "classical" bulge. Soto et al. (2007) argued that the metal-rich bulge stars exhibit a vertex deviation expected for stars orbiting in the bar, while the metal-poor $([\mathrm{Fe} / \mathrm{H}]<-0.5)$ stars could plausibly be on non-barred orbits. Hill et al. (2011) ar-

\footnotetext{
${ }^{1}$ Some results from the ARGOS study are presented in Ness et al. (2013), but the individual abundances are not yet publicly available.

${ }^{2}$ We make use of the standard spectroscopic notation where $[\mathrm{A} / \mathrm{B}] \equiv \log \left(\mathrm{N}_{\mathrm{A}} / \mathrm{N}_{\mathrm{B}}\right)_{\text {star }}-\log \left(\mathrm{N}_{\mathrm{A}} / \mathrm{N}_{\mathrm{B}}\right)_{\odot}$ and $\log \epsilon(\mathrm{A}) \equiv \log \left(\mathrm{N}_{\mathrm{A}} / \mathrm{N}_{\mathrm{H}}\right)+12.0$ for elements $\mathrm{A}$ and $\mathrm{B}$.
} 
gues strongly for bimodality, connecting two putative peaks in the $[\mathrm{Fe} / \mathrm{H}]$ distribution with differing $[\mathrm{Mg} / \mathrm{Fe}]$ ratios. Bensby et al. (2011) also find evidence for bimodality in their sample of 26 microlensed bulge dwarfs and subgiants, although they have recently increased their sample to 58 stars (Bensby et al. 2013) and now favor a multi-component model that may also incorporate the bimodal structures found in previous studies. Similarly, Ness et al. (2013), using $[\mathrm{Fe} / \mathrm{H}]$ values derived from moderate resolution spectra of a large sample $(\sim 28,000)$ of bulge giant and clump stars, deconvolve the metallicity distributions into 5 separate Gaussians that are asserted to vary systematically with Galactic latitude and longitude, and to reflect different populations. The BRAVA survey of $\sim 10,000$ bulge $\mathrm{M}$ giants (e.g., Rich et al. 2007a; Kunder et al. 2012) does not find any hint of kinematic substructure or a significant classical bulge. Shen et al. (2010) argue that very strong limits are placed on any classical bulge fraction $(\lesssim 8-15 \%$ of the disk mass) from the BRAVA survey. It has been shown theoretically possible for a rapidly rotating bar to spin up a classical bulge (e.g., Shen et al. 2010; Saha et al. 2012), but such an effect would likely be unveiled by the metal-poor stars exhibiting rapid rotation and bar-like kinematics, which is not observed.

The question of correlations between $[\mathrm{Fe} / \mathrm{H}]$, velocity, and/or velocity dispersion are among the most actively debated aspects of the substructure argument. Babusiaux et al. (2010) find a striking trend for the more metal-rich stars to have the highest velocity dispersion in Baade's Window $\left(b=-4^{\circ}\right)$; the trend reverses itself by $\mathrm{b}=-12^{\circ}$. Exploring intermediate minoraxis fields near $\mathrm{b}=-8^{\circ}$ and $-10^{\circ}$, Johnson et al. (2011) and Uttenthaler et al. (2012) find the common trend (e.g. Rich 1990) of velocity dispersion decreasing with increasing metallicity.

A further motivation to explore off-axis bulge fields addresses similarities and differences between such fields and the Galactic thick disk. In a bulge formation scenario in which the bar has thickened from a preexisting massive disk, it would not be surprising to find chemical similarities between bulge and thick disk stars; these might even be expected to predominate off-axis. Here, there is also some uncertainty. While early analyses (Zoccali et al. 2006; Fulbright et al. 2007; Lecureur et al. 2007) found that bulge stars were likely $\alpha$-enhanced, particularly in their $[\mathrm{O} / \mathrm{Fe}]$ and $[\mathrm{Mg} / \mathrm{Fe}]$ ratios, compared to the thick disk, more recent differential studies (e.g., Meléndez et al. 2008; Ryde et al. 2010; Alves-Brito et al. 2010; Gonzalez et al. 2011) have instead concluded that the thick disk and metal-poor bulge likely share identical $[\alpha / \mathrm{Fe}]$ distributions (but see also Bensby et al. 2013). However, it is not yet clear if these abundance similarities extend to super-solar metallicities and/or light odd-Z and heavy neutron-capture elements (e.g., Fulbright et al. 2007; Lecureur et al. 2007; AlvesBrito et al. 2010; McWilliam et al. 2010; Johnson et al. 2012).

Exploring the bulge in new fields, and near the bulge/halo boundary, offers significant opportunities to search for bulge substructure and to explore whether the bulge population is complex and multimodal over its whole volume. Although 
future samples will reach thousands of stars, our sample of 264 red giant branch (RGB) stars observed in multiobject mode at moderate $(\mathrm{R} \sim 18,000)$ resolution will yield new constraints on the structure and substructure of the bulge. These data will also provide new tests of whether the bulge resembles the thick disk, and the presence of kinematic or composition substructure.

\section{OBSERVATIONS AND DATA REDUCTION}

The observations for this project were obtained between 2011 August 19-20 with the WIYN 3.5m telescope at Kitt Peak National Observatory and 2011 September 8-12 with the Blanco $4 \mathrm{~m}$ telescope at Cerro Tololo Inter-American Observatory. WIYN was used to observe the northern bulge field centered near $(1, b)=(+8.5,+9)$ and the Blanco was used to observe the two southern bulge fields centered near $(1, b)=(-5.5,-7)$ and $(-4,-9)$. All spectra were acquired using the Hydra multifiber positioners and bench spectrographs. For WIYN-Hydra, we used the red fibers, 316 line $\mathrm{mm}^{-1}$ Echelle grating, red camera, and X18 filter to achieve a resolving power of $\mathrm{R}(\lambda / \Delta \lambda) \approx 18,000$. We also employed only one spectrograph setup with wavelength coverage ranging from about 6050$6350 \AA$. For Blanco-Hydra, we used the large $300 \mu \mathrm{m}\left(2^{\prime \prime}\right)$ fibers, $400 \mathrm{~mm}$ Bench Schmidt camera, 316 line $\mathrm{mm}^{-1}$ Echelle grating, and the E6257 and E6757 filters to achieve $R \approx 18,000$ in the first setup (6125$6345 \AA)$ and $R \approx 15,000$ in the second setup (6550-6840 $\AA$ ). A summary of the observation dates, instrument setups, and exposure times for each field is provided in Table 1.
The data reduction for both WIYNHydra and Blanco-Hydra data were carried out using standard IRAF tasks 3 Overscan removal and bias subtraction were accomplished using the ccdproc routine. The remaining data reduction tasks, which include fiber identification and tracing, scattered light removal, flat-field correction, ThAr wavelength calibration, cosmic-ray removal, sky subtraction, and object spectrum extraction, were carried out using the dohydra task. For the spectrograph setups that included the $\sim 6275$ $6330 \AA$ telluric absorption feature, we observed several rapidly rotating $B$ stars at various airmasses in order to remove telluric contamination in the object spectra. The final telluric correction was applied with the telluric IRAF task. All spectra were then combined via co-addition and continuum normalized by fitting a low order polynomial through the continuum points identified in Fulbright et al. (2006; their Table 5). The signal-to-noise ratio $(\mathrm{S} / \mathrm{N})$ of the combined spectra ranged from 75 to more than 300 .

\subsection{Target Selection}

Since many of the previous bulge spectroscopic analyses have focused primarily on minor-axis fields (see $\S 1$ ), we targeted fields that were located away from the minor-axis (i.e., $l \neq 0$ ). The observed fields were also selected to have relatively low interstellar extinction $(\mathrm{E}(\mathrm{B}-\mathrm{V}) \lesssim 0.35)$, in order to minimize the integration time

\footnotetext{
${ }^{3}$ IRAF is distributed by the National Optical Astronomy Observatory, which is operated by the Association of Universities for Research in Astronomy, Inc., under cooperative agreement with the National Science Foundation.
} 
needed to reach $\mathrm{S} / \mathrm{N} \gtrsim 75$. For the Blanco, 5 hours of integration with our Hydra setups yields $\mathrm{S} / \mathrm{N} \gtrsim 75$ for stars with $\mathrm{V} \lesssim 14.5$. Given the higher airmass and shorter time frame with which we were able to observe the bulge with WIYN, we selected brighter targets $(\mathrm{V} \lesssim 13.5-14)$. However, the significant throughput increase provided by the recent upgrade to the WIYN bench spectrograph (Bershady et al. 2008; Knezek et al. 2010) proved to make the selection of brighter stars an unnecessary constraint.

Unfortunately, constraining bulge membership for individual stars is often a difficult task using currently available data. Although high-quality optical photometry is not available for large regions of the bulge, the 2MASS database (Skrutskie et al. 2006) provides accurate coordinates and infrared photometry for the bright giants we were targeting. From the raw 2MASS catalog, we created an initial target list by only selecting stars which had: (1) $4000<\mathrm{T}_{\text {eff }}<4800 \mathrm{~K},(2) \mathrm{V}<14.5$, and (3) $\log (\mathrm{g})<2.5$. The temperatures were determined using the $\mathrm{J}-\mathrm{K}$ color-temperature relation provided by Alonso et al. (1999; 2001). All stars were individually dereddened using the $\mathrm{E}(\mathrm{B}-\mathrm{V})$ values derived from the Schlegel et al. (1998) dust maps. Optical V magnitudes were estimated by taking the $\mathrm{T}_{\text {eff }}$ values calculated from $\mathrm{J}-\mathrm{K}$ and then inverting the Alonso et al. (1999) $\mathrm{V}-\mathrm{K}$ color-temperature relation to solve for $\mathrm{V}$. The range of possible $\log (\mathrm{g})$ values for each star were determined by examining the temperature-gravity-color relations in Kučinskas et al. (2005; 2006) to ensure $\log (\mathrm{g})<2.5 .4$ The culled prelim-

\footnotetext{
${ }^{4}$ Since the temperature-gravity-color relations in
} Kučinskas et al. $(2005 ; 2006)$ are only calibrated inary target list of $\sim 1000-3000$ stars was then input into the Hydra fiber positioning software to produce an optimal configuration file for each field. In addition to 10-20 sky fibers for each configuration, we were able to place fibers on 91 stars for the $(-5.5,-7)$ field, 105 stars for the $(-4,-9)$ field, and 68 stars for the $(+8.5,+9)$ field.

In Figure 1 we show 2MASS colormagnitude diagrams (CMDs) for all three fields, and also identify the observed target stars. While $\sim 90 \%$ of the targets in the two southern bulge fields and all of the stars in the northern bulge field lie well on the bulge RGB, $\sim 10 \%$ of the stars in the two southern bulge fields lie in a region that is likely a mix of bulge RGB and foreground red clump stars (the approximate separation is illustrated by the dashed blue lines in Figure 1). It is possible that some of these targets are actually foreground red clump stars, but we find only a small fraction of the bluest stars to have large proper motions and estimate the contamination ratio to be $\lesssim 10 \%$ of the total sample (see $\S 4.1 .1$ ). For the majority of target stars that lie on the RGB, it is clear from Figure 1 that the observations are biased toward the bluer side of the RGB. This is a result of our choice to not select stars with $\mathrm{T}_{\text {eff }}<4000 \mathrm{~K}$, in order to avoid strong CN and $\mathrm{TiO}$ molecular features in the spectra that would prevent reliable abundance determinations via equivalent width measurements. However, we show in $§ 4.1 .1$ that we do not expect the selection of bluer stars to produce a strong metallicity bias.

up to $[\mathrm{Fe} / \mathrm{H}]=0$, we applied a linear extrapolation out to $[\mathrm{Fe} / \mathrm{H}]=+0.5$. This permits coverage from $[\mathrm{Fe} / \mathrm{H}]=-2$ to +0.5 , which spans the bulge's full metallicity range. 


\section{DATA ANALYSIS}

\subsection{Model Stellar Atmospheres}

As mentioned above, we used the dereddened $\left(\mathrm{J}-\mathrm{K}_{\mathrm{S}}\right)_{\mathrm{o}}$ color 15 for each star to derive $\mathrm{T}_{\text {eff }}$ via the Alonso et al. (1999) colortemperature relation. In order to account for interstellar extinction, we assumed the relation $\mathrm{E}\left(\mathrm{J}-\mathrm{K}_{\mathrm{S}}\right) / \mathrm{E}(\mathrm{B}-\mathrm{V})=0.505$ (Fiorucci \& Munari 2003) and used the recommended $\mathrm{E}(\mathrm{B}-\mathrm{V})$ value from Schlegel et al. (1998) 6 The average $\mathrm{E}(\mathrm{B}-\mathrm{V})$ values were $0.240(\sigma=0.036), 0.136 \quad(\sigma=0.008)$, and $0.385(\sigma=0.024)$ for the $(-5.5,-7),(-$ $4,-9)$, and $(+8.5,+9)$ fields, respectively. We did not attempt to refine $\mathrm{T}_{\text {eff }}$ using excitation equilibrium; however, a star-bystar examination of the $\log \epsilon(\mathrm{Fe} \mathrm{I})$ abundance versus excitation potential plot indicated that significant ( $\gtrsim 100 \mathrm{~K}) \mathrm{T}_{\text {eff }}$ adjustments were likely not necessary. This was true even for the most metal-rich stars $([\mathrm{Fe} / \mathrm{H}]>+0.2)$, which fall just outside the calibration range of the Alonso et al. (1999) J-K color-temperature relation. In a more quantitative sense, the average, median, and standard deviations of the slopes for the $\log \epsilon(\mathrm{Fe} \mathrm{I})$ versus excitation potential plots were: $-0.006,-0.011$, and 0.043 for the $(-5.5,-7)$ field, +0.002 , -0.004 , and 0.032 for the $(-4,-9)$ field, and $-0.019,-0.023$, and 0.037 for the $(+8.5,+9)$ field. For comparison, altering $\mathrm{T}_{\text {eff }} \pm 100 \mathrm{~K}$ in a "typical" bulge giant with $[\mathrm{Fe} / \mathrm{H}]=-$ 0.3 is expected to produce a change in the magnitude of the slope by $\sim 0.04$ at

\footnotetext{
${ }^{5}$ The 2MASS $\mathrm{J}$ and $\mathrm{K}_{\mathrm{S}}$ values were converted onto the TCS system, as required for using the Alonso et al. (1999) color-temperature relation.

${ }^{6}$ The $\mathrm{E}(\mathrm{B}-\mathrm{V})$ values can be accessed at: http://irsa.ipac.caltech.edu/applications/DUST/.
}

$\mathrm{T}_{\text {eff }}=4200 \mathrm{~K}$ and $\sim 0.02$ at $\mathrm{T}_{\text {eff }}=4800 \mathrm{~K}$.

Surface gravities were derived by interpolating within the $11 \mathrm{Gyr}$ isochrones provided by the Dartmouth Stellar Evolution Database (Dotter et al. 2007) 7 We initially assumed a level of $\alpha$-enhancement that followed the $[\alpha / \mathrm{Fe}]$ versus $[\mathrm{Fe} / \mathrm{H}]$ trends of Gonzalez et al. (2011) and Johnson et al. (2011). However, after measuring $[\alpha / \mathrm{Fe}]$ for each star we reinterpolated using a grid with the appropriate level of $\alpha$-enhancement. As can be seen in Figure 2, our assumed $\log (\mathrm{g})$ values are typically in good agreement with other $\log (\mathrm{g})$ estimates of bulge RGB stars in the literature. Note that the literature values shown in Figure 2 represent a variety of techniques for deriving $\log (\mathrm{g})$, including ionization equilibrium, isochrone fitting, and photometric estimates.

For each star the model atmosphere metallicity was set at $[\mathrm{M} / \mathrm{H}]=-0.3$ and then adjusted to equal the average derived $[\mathrm{Fe} / \mathrm{H}]$ ratio. Similarly, the microturbulence (vt) was set at $2 \mathrm{~km} \mathrm{~s}^{-1}$ and then adjusted so that the plot of Fe I abundance versus line strength produced a zero slope. For all stars the model atmosphere was calculated by interpolating within the $\alpha$-rich AODFNEW ATLAS9 grid (Castelli et al. 1997) 8 While not all of the stars in our sample are $\alpha$-rich, Fulbright et al. (2007) found that the impact of using the solar scaled versus $\alpha-$ rich ATLAS9 models on derived abundances was relatively small for bulge giants $(\lesssim 0.1$ dex in most cases). Specifi-

\footnotetext{
${ }^{7}$ The isochrones can be accessed at: http://stellar.dartmouth.edu/ models/grid.html

${ }^{8}$ The model atmosphere grid can be accessed at: http://wwwuser.oat.ts.astro.it/castelli/grids.html
} 
cally, the average effect on $[\mathrm{Fe} / \mathrm{H}],[\mathrm{O} / \mathrm{Fe}]$, $[\mathrm{Si} / \mathrm{Fe}]$, and $[\mathrm{Ca} / \mathrm{Fe}]$ was found to be (in the sense AODFNEW-ODFNEW): +0.06 $(\sigma=0.03),-0.05(\sigma=0.07),+0.02(\sigma=0.03)$, and $-0.02(\sigma=0.03)$, respectively. We performed the same test on our data set and found that the average AODFNEWODFNEW abundance change for $[\mathrm{Fe} / \mathrm{H}]$, $[\mathrm{O} / \mathrm{Fe}], \quad[\mathrm{Si} / \mathrm{Fe}]$, and $[\mathrm{Ca} / \mathrm{Fe}]$ was similar at: $+0.05(\sigma=0.07),+0.06(\sigma=0.06)$, $+0.10(\sigma=0.03)$, and $-0.10(\sigma=0.03)$, respectively. The final model atmosphere parameters, photometry, and $\mathrm{E}(\mathrm{B}-\mathrm{V})$ values are provided in Table 2 .

\subsection{Equivalent Width Abundance Determinations}

The Fe I abundances were determined by measuring equivalent widths (EWs), using software developed for Johnson et al. (2008). The final $[\mathrm{Fe} / \mathrm{H}]$ abundances, listed in Tables 2 and 3, were derived using the 2010 version of the LTE line analysis code MOOG (Sneden 1973). Single, isolated lines were fit with a Gaussian profile and moderately blended lines were deblended with up to five Gaussian profiles. We selected $61 \mathrm{Fe} I$ lines located in the wavelength regions specified in $\S 2$ that were relatively isolated in the spectra of both Arcturus (Hinkle et al. 2000) 9 and $\mu$ Leo (Moultaka et al. 2004) 10 On average, the $[\mathrm{Fe} / \mathrm{H}]$ abundances for the $(-5.5,-7)$, $(-4,-9)$, and $(+8.5,+9)$ fields are based on $21 \mathrm{Fe} I$ lines. The number of lines used

${ }^{9}$ The Arcturus atlas can be accessed at: http://www.noao.edu/archives.html.

${ }^{10}$ Based on spectral data retrieved from the ELODIE archive at Observatoire de HauteProvence (OHP). The ELODIE archive can be accessed at: http://atlas.obs-hp.fr/elodie/. for each star is less than the 61 potential lines due to variations in wavelength coverage (Blanco-Hydra versus WIYN-Hydra), temperature, metallicity, and S/N. The average line-to-line dispersion of $0.16 \mathrm{dex}$ $(\sigma=0.03)$ was found to not vary significantly as a function of temperature or metallicity, and is reasonable given the relatively high metallicity and cool temperatures of our target stars coupled with the moderate spectral resolution.

The Fe I abundances were determined on a line-by-line basis relative to Arcturus. The oscillator strength (log gf) values listed in Table 4 were derived by measuring the EW of each line in the high resolution, high $\mathrm{S} / \mathrm{N}$ Arcturus atlas and forcing the derived abundance for each line to be $[\mathrm{Fe} / \mathrm{H}]=-0.50$, assuming the Arcturus model atmosphere parameters given in Fulbright et al. (2006; $\mathrm{T}_{\text {eff }}=4290 \mathrm{~K}, \log (\mathrm{g})=1.60,[\mathrm{Fe} / \mathrm{H}]=-0.50$, and $\left.\mathrm{vt}=1.67 \mathrm{~km} \mathrm{~s}^{-1}\right)$. The wavelength and excitation potential values given in Table 4 are from the NIST Atomic Spectra Database (Ralchenko et al. 2011)11, Vienna Atomic Line Database (VALD; Kupka et al. 2000)12, or Thevenin (1990). Sample spectra of both the 6250 and 6700 $\AA$ setups for stars with similar $\mathrm{T}_{\text {eff }}$ but different $[\mathrm{Fe} / \mathrm{H}]$ are shown in Figure 3, This figure also illustrates data quality as well as the change in line strength, continuum availability, and molecular contamination as a function of $[\mathrm{Fe} / \mathrm{H}]$.

\footnotetext{
${ }^{11}$ The NIST database can be accessed at: http://www.nist.gov/pml/data/asd.cfm.

${ }^{12}$ VALD can be accessed at: http://vald.astro.univie.ac.at/ vald/php/vald.php
} 


\subsection{Spectrum Synthesis Abundance Determinations}

For the elements other than Fe, we determined abundances via spectrum synthesis rather than EW measurement. Given the large number of stars analyzed here, we employed the computationally parallel version of the MOOG synth driver developed for Johnson et al. (2012). Similar to the Fe abundance measurements, $[\mathrm{O} / \mathrm{Fe}],[\mathrm{Si} / \mathrm{Fe}]$, and $[\mathrm{Ca} / \mathrm{Fe}]$ ratios were determined relative to Arcturus. We adopted the Arcturus abundances derived in Fulbright et al. (2007): $[\mathrm{O} / \mathrm{Fe}]=+0.48,[\mathrm{Si} / \mathrm{Fe}]=+0.35$, and $[\mathrm{Ca} / \mathrm{Fe}]=+0.21$. These adopted values are within $\sim 0.1$ dex of those derived recently by Ramírez \& Allende Prieto (2011). The lines used here are listed in Table 3.

For the $6300 \AA$ [O I] line we synthesized the region spanning 6295-6305 A, including the $6300.34 \AA \mathrm{Ni}$ I line that is blended with the 6300.30 [O I] feature. We assumed $[\mathrm{Ni} / \mathrm{Fe}]=0$ for all syntheses. The initial line list was compiled from VALD for atomic lines and the Kurucz databas 13 for $\mathrm{CN}$ molecular lines. The final log gf values for all significant atomic and molecular lines in the $6295-6305 \AA$ region were adjusted to minimize the difference between our synthetic spectrum and the Arcturus atlas. In order to fit the $\mathrm{CN}$ features, we adopted the Arcturus carbon and nitrogen abundances from Peterson et al. (1993; $[\mathrm{C} / \mathrm{Fe}]=+0.0$ and $[\mathrm{N} / \mathrm{Fe}]=+0.3)$. In general this provided a satisfactory fit to the $\mathrm{CN}$ lines using the original Kurucz line list and only a few minor adjustments were required. For the Sc I line that is blended

${ }^{13}$ The Kurucz line list database can be accessed at: http://kurucz.harvard.edu/linelists.html. with the [O I] feature in our Hydra spectra but not the Arcturus atlas, we set the log gf value assuming $[\mathrm{Sc} / \mathrm{Fe}]=+0.2$ (Peterson et al. 1993). However, as can be seen in the sample syntheses provided in Figure 4, there is enough separation between the $[\mathrm{O}$ I] and Sc features in the Hydra spectra to reasonably account for the Sc contribution.

Since the [O I] line is both modestly affected by $\mathrm{CN}$ blending (see bottom panels of Figure (4) and also by the $\mathrm{C}+\mathrm{N}$ abundance (via the molecular equilibrium calculation), we identified a small window in the $6250 \AA$ spectra that could be used to roughly estimate the $\mathrm{CN}$ abundance. While there are other similar windows that may be used, especially in the $6700 \AA$ spectra, we selected the 6331-6339 $\AA$ window (shown in Figure 5) because it provided a several Angstrom wide region comprised of mostly $\mathrm{CN}$ lines and was observed with both the WIYN and Blanco bench spectrographs. For each star we initially set $[\mathrm{C} / \mathrm{Fe}]=-0.30$ and $[\mathrm{N} / \mathrm{Fe}]=+0.50$, values typical for bulge RGB stars (e.g., Meléndez et al. 2008; Ryde et al. 2010), and then adjusted $[\mathrm{N} / \mathrm{Fe}]$ until a satisfactory fit was found. The derived $[\mathrm{C} / \mathrm{Fe}]$ and $[\mathrm{N} / \mathrm{Fe}]$ abundances were then used in the [O I] synthesis. This processes was iterated at least once for each star to account for the correlated variations in line strength between $\mathrm{CN}$ and $\mathrm{O}$.

The Si and $\mathrm{Ca}$ abundances are based on synthesis fits to lines in the $6140-6170 \AA$ window. In order to properly account for $\mathrm{CN}$ contamination, $\mathrm{Si}$ and $\mathrm{Ca}$ were measured after obtaining CNO abundances. For the few stars where $[\mathrm{O} I]$ could not be measured, we have adopted the general $[\mathrm{O} / \mathrm{Fe}]$ versus $[\mathrm{Fe} / \mathrm{H}]$ trend found in 
our data (see §4.3). Sample Si and Ca synthesis fits for a cool, metal-rich giant are shown in Figure 6. While most of the lines are not strongly affected by $\mathrm{CN}$, the $6155 \AA \mathrm{Si}$ I line is sensitive to the $\mathrm{CN}$ abundance in our most metal-rich giants. As can be seen in Figure 6, changing the $\mathrm{CN}$ abundance by \pm 0.3 dex can result in a Si abundance uncertainty $\gtrsim 0.2$ dex. A comparison between the $6155 \AA$ Si abundance and the $6145 \AA$ Si abundance, which is only weakly affected by $\mathrm{CN}$, suggests that at least on average there is reasonable agreement with $\left\langle[\mathrm{Si} / \mathrm{Fe}]_{6155}-[\mathrm{Si} / \mathrm{Fe}]_{6145}\right\rangle=-$ $0.03(\sigma=0.17)$. The four Ca lines used here exhibit a similar degree of agreement with an average line-to-line dispersion of 0.08 $\operatorname{dex}(\sigma=0.05)$.

\subsection{Abundance Uncertainty Esti- mates}

Tables 5a-5d summarize the abundance uncertainty estimates for $\log \epsilon(\mathrm{Fe})$, $\log \epsilon(\mathrm{O}), \log \epsilon(\mathrm{Si})$, and $\log \epsilon(\mathrm{Ca})$ due to changes in $\mathrm{T}_{\text {eff }}+100 \mathrm{~K}, \log (\mathrm{g})+0.30$, $[\mathrm{M} / \mathrm{H}]+0.30$, and $\mathrm{vt}+0.30 \mathrm{~km} \mathrm{~s}^{-1}$. The $\sigma / \sqrt{ }(\mathrm{N})$ values for each element are also given as an estimate of the measurement uncertainty. For elements in Tables 5b-5d where only one line was available for measurement (e.g., [O I]), a value of $\sigma / \sqrt{ }(\mathrm{N})=0.05$ has been assigned. This represents the average value for cases where multiple lines could be measured, and is a reasonable uncertainty estimate for visually fitting synthetic spectra. The total error column in Tables 5a-5d reflects the $\mathrm{T}_{\text {eff }}, \log (\mathrm{g}),[\mathrm{M} / \mathrm{H}]$, vt, and measurement uncertainties added in quadrature, and are reflected in the error bars of all subsequent figures.
The abundance sensitivities to each model atmosphere parameter were calculated by varying each parameter independently. The procedure involved first using the best-fit model atmosphere parameters and abundances given in Tables 2-3 to calculate theoretical EWs for each line. These theoretical EWs were held fixed and then a new model atmosphere was created with the $\mathrm{T}_{\text {eff }}, \log (\mathrm{g}),[\mathrm{M} / \mathrm{H}]$, or $\mathrm{vt}$ value varied by the amount given in Tables $5 \mathrm{a}-5 \mathrm{~d}$. However, it should be noted that the sensitivity of each line to changes in model atmosphere parameters are not necessarily the same. Therefore, the values given in Tables $5 \mathrm{a}-5 \mathrm{~d}$ represent the average difference between the newly derived abundances from each line and the original abundance given in Table 2 .

The uncertainty ranges of $\Delta \mathrm{T}_{\text {eff }}=+100$ $\mathrm{K}, \Delta \log (\mathrm{g})=+0.30, \Delta[\mathrm{M} / \mathrm{H}]=+0.30$, and $\Delta \mathrm{vt}=+0.30 \mathrm{~km} \mathrm{~s}^{-1}$ are likely to be conservative upper limits for each parameter. The $\mathrm{T}_{\text {eff }}$ variations, based on the uncertainty in $\mathrm{E}(\mathrm{B}-\mathrm{V})$, are typically small $(<50 \mathrm{~K})$ for almost all stars in our sample. In particular, the average differences between the $\mathrm{T}_{\text {eff }}$ derived assuming the recommended $\mathrm{E}(\mathrm{B}-\mathrm{V})$ and the maximum/minimum $\mathrm{E}(\mathrm{B}-\mathrm{V})$ values are: +38 $\mathrm{K}(\sigma=27 \mathrm{~K}) /-24 \mathrm{~K}(\sigma=15 \mathrm{~K})$ for the $(-5.5,-7)$ field, $+14 \mathrm{~K}(\sigma=7 \mathrm{~K}) /-10 \mathrm{~K}$ $(\sigma=6 \mathrm{~K})$ for the $(-4,-9)$ field, and +21 $\mathrm{K}(\sigma=12 \mathrm{~K}) /-22 \mathrm{~K}(\sigma=10 \mathrm{~K})$ for the $(+8.5,+9)$ field. These values are significantly smaller than the $125 \mathrm{~K} 1 \sigma$ value of the Alonso et al. (1999) J-K $\mathrm{K}_{\mathrm{S}}$ colortemperature calibration. When we compare the $T_{\text {eff }}$ values derived using the Alonso et al. (1999) calibration to those derived using the González Hernández \& 
Bonifacio (2009) calibration, we find our adopted Alonso et al. (1999) temperatures to be cooler by $63 \mathrm{~K}(\sigma=8 \mathrm{~K})$ in the ($5.5,-7)$ field, $68 \mathrm{~K}(\sigma=7 \mathrm{~K})$ in the $(-4,-9)$ field, and $46 \mathrm{~K}(\sigma=13 \mathrm{~K})$ in the $(+8.5,+9)$ field. Therefore, we believe that an estimated uncertainty of $100 \mathrm{~K}$ is a reasonable assumption for our data.

Since we did not calculate $\log (\mathrm{g})$ directly from photometry or ionization equilibrium, it is difficult to assess the true $\log (\mathrm{g})$ uncertainty. However, examination of Figure 2 suggests that the difference between our derived $\log (\mathrm{g})$ values and those in the literature is typically within \pm 0.30 . For comparison, the Bescancon mode 14 (Robin et al. 2003) predicts that most bulge stars along our observed lines-ofsight should vary in distance by approximately $\pm 2 \mathrm{kpc}$, though the full range may extend several kpc on either side of the Galactic center. This would lead to a maximum photometric $\log (\mathrm{g})$ uncertainty of $\sim 0.20-0.25$. Note that oxygen, especially when normalized to Fe I, is the element most affected by $\log (\mathrm{g})$ uncertainties. Similarly, since surface gravity is proportional to $\log \left(\mathrm{M} / \mathrm{M}_{\odot}\right)$ for stars with the same $\mathrm{T}_{\text {eff }}$ but different mass, it is likely that our abundance ratios will not be significantly altered by the possible inclusion of lower mass AGB stars in our sample.

As stated previously, the average lineto-line dispersion for Fe I is 0.16 dex, with a small dispersion of 0.03 dex. Similarly, we found that the use of $\alpha$-enhanced versus solar-scaled models affected abundance ratios at $\lesssim 0.1$ dex level. Therefore, it seems likely that the use of a model

${ }^{14}$ The Bescancon model form can be accessed at: http://model.obs-besancon.fr/. atmosphere $[\mathrm{M} / \mathrm{H}]$ uncertainty of 0.3 dex should represent a conservative upper limit on the effects continuous opacity and electron number density have on derived abundances. From Tables 5a-5d, we find that $[\mathrm{M} / \mathrm{H}]$ uncertainties have the largest effect ( $\sim 0.1$ dex in magnitude) on $\mathrm{O}$ and Fe while $\mathrm{Si}$ and $\mathrm{Ca}$ abundances are less sensitive. The magnitude of the $[\mathrm{M} / \mathrm{H}]$ uncertainty effect also tends to increase with increasing metallicity.

Our adopted microturbulence uncertainty of $0.3 \mathrm{~km} \mathrm{~s}^{-1}$ may also be a conservative estimate. For an individual star, the typical vt uncertainty based on removing any trends in Fe I abundance versus line strength is $\sim 0.1-0.15 \mathrm{~km} \mathrm{~s}^{-1}$. When comparing stars of similar metallicity (in 0.5 dex bins), the $1 \sigma$ variation in derived vt values for stars of comparable temperature ranges from $0.17 \mathrm{~km} \mathrm{~s}^{-1}$ in the most metal-poor stars to $0.22 \mathrm{~km} \mathrm{~s}^{-1}$ in the most metal-rich stars. The effect of vt uncertainty on abundance is clear in Tables $5 \mathrm{a}-5 \mathrm{~d}$, which are sorted by metallicity in each field. Microturbulence becomes an increasingly important factor with increasing metallicity and thus line strength. Similarly, elements that were derived from stronger lines (e.g., Fe and $\mathrm{Ca}$ ) are most sensitive to vt uncertainty.

\subsection{Radial Velocity Determinations}

Radial velocities for all stars were measured using the fxcor task in IRAF. For templates, we calculated synthetic spectra over the full wavelength range covered for each star. The synthetic spectra were produced using the model atmosphere parameters given in Table 2, and were smoothed and binned to match the dispersion of the 
object spectra. The average measurement error returned by fxcor was similar for all fields: $0.71 \mathrm{~km} \mathrm{~s}^{-1}\left(\sigma=0.48 \mathrm{~km} \mathrm{~s}^{-1}\right), 0.88$ $\mathrm{km} \mathrm{s}^{-1}\left(\sigma=0.53 \mathrm{~km} \mathrm{~s}^{-1}\right)$, and $0.62 \mathrm{~km}$ $\mathrm{s}^{-1}\left(\sigma=0.38 \mathrm{~km} \mathrm{~s}^{-1}\right)$ for the $(-5.5,-7)$, ($4,-9)$, and $(+8.5,+9)$ fields, respectively. The IRAF task rvcorrect was used the determine the heliocentric correction for all stars. The derived heliocentric radial velocities are provided in Table 2.

\section{RESULTS AND DISCUSSION}

\subsection{Metallicity Distribution Func- tions}

In Figure 7 we show the derived metallicity distribution functions for all three off-axis fields. These metallicity distributions are in reasonable agreement with those derived for similar outer bulge $\left(|\mathrm{b}|>4^{\circ}\right)$ minor-axis fields (Zoccali et al. 2008; Johnson et al. 2011; Ness et al. 2013; Uttenthaler et al. 2012). For the $(-5.5,-7),(-4,-9)$, and $(+8.5,+9)$ fields, we find a full range in $[\mathrm{Fe} / \mathrm{H}]$ that spans -1.50 to $+0.66 \operatorname{dex}(\sigma=0.47$ dex $),-1.40$ to $+0.26 \operatorname{dex}(\sigma=0.42 \mathrm{dex})$, and -1.26 to +0.59 dex $(\sigma=0.44 \mathrm{dex})$, respectively. As is the case with previous high resolution spectroscopic studies, we do not find any stars with $[\mathrm{Fe} / \mathrm{H}] \lesssim-1.5$ that may be present in the bulge from the earliest epoch of star formation. This is in agreement with the ARGOS sample by Ness et al. (2013) that only found $0.71 \%(100 / 14147)$ of stars within $3.5 \mathrm{kpc}$ of the Galactic center to have $[\mathrm{Fe} / \mathrm{H}]<-1.5$.

The observed vertical metallicity gradient present along the bulge minor-axis, at least for $|\mathrm{b}|>4^{\circ}$ (Zoccali et al. 2008), appears to also be present in off-axis fields.
This result is illustrated in Figure 8, where we show the metallicity distribution functions for four minor-axis and four off-axis fields at similar Galactic latitudes. In a similar fashion to the minor-axis trend, the median $[\mathrm{Fe} / \mathrm{H}]$ ratios for the $(+5,-3)$ field given in Gonzalez et al. (2011) and our ($5.5,-7)$ and $(-4,-9)$ fields are $[\mathrm{Fe} / \mathrm{H}]=-0.08$, -0.29 , and -0.44 , respectively. The decline in the median $[\mathrm{Fe} / \mathrm{H}]$ ratio with increasing Galactic latitude appears to be driven by the same phenomenon in both the minoraxis and off-axis fields. In particular, both sets of observations show a decrease in the relative fraction of metal-rich $([\mathrm{Fe} / \mathrm{H}] \gtrsim 0)$ stars, an increase in the relative fraction of the metal-poor $([\mathrm{Fe} / \mathrm{H}] \lesssim-1)$ stars, and no significant change in the relative fraction of intermediate metallicity stars as a function of increasing Galactic latitude. This result is at least qualitatively in agreement with the scenario proposed by Babusiaux et al. (2010), in which the metallicity gradient is a reflection of the change in the bulge/bar population mixture at different Galactic latitudes (but see also Ness et al. 2013).

The data presented above indicate that the vertical metallicity gradient along $\mathrm{l} \sim \pm 5^{\circ}$ is approximately $0.4 \mathrm{dex} \mathrm{kpc}^{-1}$, which is similar to the minor-axis gradients found by Zoccali et al. (2008; 0.6 dex $\mathrm{kpc}^{-1}$ ) and Ness et al. (2013; 0.45 dex $\left.\mathrm{kpc}^{-1}\right)$. Given the uncertainty in

\footnotetext{
${ }^{15}$ Note that Ness et al. (2013) also argue that the metallicity gradient of the individual bulge components they have identified may be significantly more shallow, on the order of $0.07 \mathrm{dex} \mathrm{kpc}^{-1}$, than the $0.4-0.6$ dex $\mathrm{kpc}^{-1}$ found here and in previous studies. Unfortunately, our sample sizes are insufficient to attempt a similar multi-component deconvolution of the distribution functions shown
} 
bulge membership and possible biases of incomplete samples, it seems unlikely that the difference in magnitude $(\sim 0.2$ dex $)$ between the derived metallicity gradients found here and in Zoccali et al. (2008) is significant. Additionally, there does not seem to be a strong radial $[\mathrm{Fe} / \mathrm{H}]$ gradient in the bulge.

Interestingly, the northern bulge $(+8.5,+9)$ field is slightly more metal-rich than the two southern fields, with a median $[\mathrm{Fe} / \mathrm{H}]=-0.23$. However, as can be seen in Figure 7 , where we plot the cumulative $[\mathrm{Fe} / \mathrm{H}]$ distribution functions for all fields, it is clear that the the $(+8.5,+9)$ and $(-5.5,-7)$ fields share similar distributions. This is supported by the results of two-sided Kolmogorov-Smirnov (KS) tests (Press et al. 1992), which indicate that there is insufficient evidence to reject the null hypothesis that the $(+8.5,+9)$ and $(-5.5,-7)$ are drawn from the same parent population $(\mathrm{p}-$ value $=0.881)$ 16. On the other hand, the $(-5.5,-7) /(-4,-9)$ and $(-4,-9) /(+8.5,+9)$ field combinations are possibly drawn from different parent populations, with p-values of 0.031 and 0.045 , respectively. It is not clear if this signifies an asymmetry in the bulge metallicity distribution between the northern and southern regions or is simply a result of the smaller sample size and slightly redder colors of the observed stars in the $(+8.5,+9)$ field (see Figure 1). We also consider the possibility that the higher metallicity of the $(+8.5,+9)$ field could be due to a predominance of the $\mathrm{X}$-shaped bulge structure in that region. Ness et al. (2012)

in Figure 7

${ }^{16}$ We adopt the common interpretation that the null hypothesis can be rejected if $\mathrm{p}<0.05$. and Uttenthaler et al. (2012) suggest that the $\mathrm{X}$-shaped bulge, traced by the double red clump, may be dominated by metalrich $([\mathrm{Fe} / \mathrm{H}]>-0.5)$ stars. As can be seen in Saito et al. (2011; their Figure 3), one of the red clump components in the $\mathrm{X}-$ shaped structure appears prominently near our $(+8.5,+9)$ sight line. A distant, lower density component is also noted near the $(-5.5,-7)$ sight line, and the higher metallicity of both our $(-5.5,-7)$ and $(+8.5,+9)$ could partially reflect the predominance of the $\mathrm{X}$-shaped structure in these fields.

\subsubsection{Sample Selection Bias and Fore- ground Contamination}

As mentioned in $\S 2.1$, the targets in all three bulge fields were selected from the blue side of the RGB to avoid cool stars with spectra that may be dominated by molecular bands (especially $\mathrm{TiO}$ ). The naive interpretation is that this will produce a strong bias in our derived metallicity distribution functions and significantly undersample the most metal-rich stars. However, the bias may not be as strong as expected because several factors combine to disperse the observed $\mathrm{J}-\mathrm{K}_{\mathrm{S}}-[\mathrm{Fe} / \mathrm{H}]$ relation including: differential reddening, a complicated spatial geometry with a distance spread that could range from $\sim 4.5$ $11.5 \mathrm{kpc}$ in our field 17 , metallicity dependent $[\alpha / \mathrm{Fe}]$ variations, a possible age range of $\sim 2-13$ Gyr for stars with $[\mathrm{Fe} / \mathrm{H}] \gtrsim-0.4$ (e.g., see Figures 15-16 of Bensby et al. 2013; but see also Clarkson et al. 2008), and the mixing of RGB and AGB stars.

In general, it is not a trivial matter to estimate the observational bias of the

\footnotetext{
${ }^{17}$ This estimate is based on the Bescancon model.
} 
color-metallicity relation in the bulge, especially in the $\mathrm{J}-\mathrm{K}_{\mathrm{S}}$ and $\mathrm{K}_{\mathrm{S}}$ dimensions, because of large uncertainties in several of the factors listed above. This is evident both in the metallicity distribution functions produced here (Figure 7), which are not dominated by metal-poor stars as one might expect, and in the $\mathrm{J}-\mathrm{K}_{\mathrm{S}}-[\mathrm{Fe} / \mathrm{H}]$ relations of previous studies that span a broader color range. In Figure 9 we show $\mathrm{K}_{\mathrm{S}}$ versus $\mathrm{J}-\mathrm{K}_{\mathrm{S}}$ color-magnitude diagrams for the Zoccali et al. (2008) and Gonzalez et al. (2011) data sets and overplot the observed stars, with the most metal-poor $([\mathrm{Fe} / \mathrm{H}]<-0.7)$ and metal-rich $([\mathrm{Fe} / \mathrm{H}]>+0.2)$ subsets identified. While the differential reddening and distance distributions vary from field-to-field, a common feature among all fields is the significant mixing of metal-poor and metal-rich stars on the giant branch. The metal-rich population in particular appears to span the entire color range, although an unknown fraction of these stars may be disk and/or halo contaminators (see below).

What is the effect on the derived metallicity distribution functions by preferentially sampling the blue side of the bulge giant branch? We can derive some estimate with the Zoccali et al. (2008) and Gonzalez et al. (2011) fields by comparing the metallicity distribution functions of the bluest third, which roughly matches our sample selection, to the full samples. The results are shown in Figure 10] where we plot histograms, cumulative distribution functions, provide results for two-sided KS tests, compare the median $[\mathrm{Fe} / \mathrm{H}]$ values, and compare the interquartile range (IQR) for each population. While it is clear from the cumulative dis- tribution functions that (in most cases) the blue samples are moderately more metalpoor than the full samples, the effect is significantly smaller than anticipated. In fact, the two-sided KS tests for all fields indicate that we cannot strongly reject the null hypothesis that the blue and full samples were drawn from the same parent population. Furthermore, the general shapes of the full metallicity distribution functions are preserved in the blue samples, and the median $[\mathrm{Fe} / \mathrm{H}]$ and IQR values do not vary significantly between the two subsets, especially in the $b>-4^{\circ}$ fields that are most similar to our fields. In other words, the qualitative and quantitative results found especially by Zoccali et al. (2008), and particularly with regard to the $[\mathrm{Fe} / \mathrm{H}]$ gradient, would not have been significantly altered by the selection of stars on the blue side of the giant branch 18 .

Finally, we can compare our $(-5.5,-7)$ and $(-4,-9)$ fields with the data presented in Ness et al. (2013; their Figure 8). For the similar latitude minor-axis fields of $(0,-7.5 ; 690$ stars $)$ and $(0,-10 ; 650$ stars $)$ in Ness et al. (2013), they find median $[\mathrm{Fe} / \mathrm{H}]$ values of -0.34 and -0.46 , respectively. This is in good agreement with the median $[\mathrm{Fe} / \mathrm{H}]$ values of our $(-5.5,-7)$ and $(-4,-9)$ fields, which have $[\mathrm{Fe} / \mathrm{H}]=-$ 0.29 and -0.44 , respectively. The $<0.05$ dex difference between our derived median $[\mathrm{Fe} / \mathrm{H}]$ values and Ness et al. (2013) is in agreement with the estimated bias introduced by the selection of stars on the blue

\footnotetext{
${ }^{18}$ We tested for the effects of differential reddening and photometric errors in the 2MASS $\mathrm{J}$ and $\mathrm{K}_{\mathrm{S}}$ data but did not find significant changes to the distribution functions or median $[\mathrm{Fe} / \mathrm{H}]$ and IQR values.
} 
side of the giant branch (see the middle panels of Figure 10). We conclude that our selection of bluer stars likely does not introduce a strong metallicity bias, and that the derived metallicity distribution functions are representative of the underlying population in each field. However, the metal-rich stars in our sample will be more concentrated on the near side of the bulge/bar system than a selection of stars with a broader color distribution.

In addition to the properties mentioned above that can blur the color-metallicity relation, contamination by disk and halo stars along the line-of-sight can also affect the derived metallicity distribution functions. While most studies agree that the halo contamination is small $(\lesssim 2 \%$; e.g., Zoccali et al. 2008; Hill et al. 2011; Uttenthaler et al. 2012), a true estimate of the (primarily) foreground thin and thick disk contamination is difficult to ascertain. In an attempt to gauge the impact of foreground contamination, the coordinates of stars in our two southern bulge fields were cross-referenced with the Southern Proper Motion IV catalog (SPM4; Girard et al. 2011)19. We were able to match $77 / 91$ stars in the $(-5.5,-7)$ field and 98/105 stars in the $(-4,-9)$ field. Histograms of the proper motion results are shown in the top two panels of Figure 11. As can be seen in Figure 11, both fields contain a dominant population with a total proper motion $\lesssim 20$ mas year ${ }^{-1}$ and a tail that extends out to $\sim 35$ mas year $^{-1}$ for the $(-5.5,-7)$ field and $\sim 60$ mas year $^{-1}$ for the $(-4,-9)$ field. This is in good agreement with that found by

${ }^{19}$ The stars in our northern bulge field have a declination of $\sim-16^{\circ}$, which is outside the SPM4 range of $-20^{\circ}$ to $-90^{\circ}$.
Uttenthaler et al. (2012; their Figure 3) at $(0,-10)$.

As a rough estimate of contamination, we can assign targets lying in the tails of the proper motion distributions as possible foreground stars. Using a discriminator of 22 mas year $^{-1}$ for the $(-5.5,-7)$ field and 21 mas year ${ }^{-1}$ for the $(-4,-9)$ field suggests foreground contamination rates of $9 \%(7 / 77)$ and $11 \%$ (11/98), respectively. However, given that many of the "outliers" fall within a few mas year ${ }^{-1}$ of our cutoff and the proper motion errors are generally $\sim 3-8$ mas year $^{-1}$, it is possible that as many as $50 \%$ of the possible outliers are actually bulge members. Additionally, since Uttenthaler et al. (2012) find that the double red clump and dual peaked metallicity distribution function features are present in their sample of stars with proper motions $>20$ mas year ${ }^{-1}$, we follow their assessment and have not excluded any stars in our sample. We do note however that the middle and bottom panels of Figure 11. which plot $(\mathrm{J}-\mathrm{K})_{\mathrm{o}}$ and $[\mathrm{Fe} / \mathrm{H}]$ versus total proper motion, give some indications that the more extreme proper motion outliers may clump together at colors and metallicities consistent with the foreground thin disk and metal-poor thick disk (or metalrich halo).

\subsection{Radial Velocities}

In Figure 12 we plot histograms of the heliocentric radial velocity distributions for all fields analyzed here. The mean, median, and standard deviations are -26.6, -29.9 , and $73.7 \mathrm{~km} \mathrm{~s}^{-1}$ for the $(-5.5,-7)$ field, $-40.7,-49.3$, and $85.1 \mathrm{~km} \mathrm{~s}^{-1}$ for the $(-4,-9)$ field, and $-3.8,+15.2$, and 81.6 $\mathrm{km} \mathrm{s}^{-1}$ for the $(+8.5,+9)$ field. Similarly, 
the mean/median galactocentric velocities $\left(\mathrm{V}_{\mathrm{GC}}\right)$ for these fields are: $-40.8 /-44.8 \mathrm{~km}$ $\mathrm{s}^{-1},-48.0 /-57.8 \mathrm{~km} \mathrm{~s}^{-1}$, and $+40.5 /+60.2$ $\mathrm{km} \mathrm{s}^{-1}$. For the two southern bulge fields, the velocity distributions and dispersions are in good agreement with the BRAVA results (e.g., see Kunder et al. 2012; their Figure 11). This is confirmed in Figure 13 where we plot the velocity distributions from this work and overlapping or nearby BRAVA fields. A two-sided KS test finds that the data are insufficient to strongly reject the null hypothesis that the $(-5.5,-7) /(-6,-7)$ fields $(\mathrm{p}-$ value $=0.063)$ and $(-4,-9) /(-4,-8)$ fields $(\mathrm{p}-$ value $=0.094)$ are drawn from the same parent populations. Additionally, the median galactocentric radial velocity and dispersion for our two southern bulge fields are in reasonable agreement with the Shen et al. (2010) bulge model, which find $\mathrm{V}_{\mathrm{GC}}=-52.2$ $(\sigma=78.9) \mathrm{km} \mathrm{s}^{-1}$ and $\mathrm{V}_{\mathrm{GC}}=-54.1(\sigma=78.2)$ $\mathrm{km} \mathrm{s}^{-1}$ at $(1, \mathrm{~b})=(-5.5,-7)$ and $(-4,-9)$, respectively (Z. Li \& J. Shen 2012; priv. comm.).

While the BRAVA sample did not extend to our single northern bulge field, the mean galactocentric velocity and dispersion we derived for the $(+8.5,+9)$ field $\left(\mathrm{V}_{\mathrm{GC}}=+40.5 \mathrm{~km} \mathrm{~s}^{-1}, \sigma=81.6 \mathrm{~km} \mathrm{~s}^{-1}\right)$ is in moderately good agreement with that found by Minitti (1996) for the nearby $(+8,+7)$ field $\left(\mathrm{V}_{\mathrm{GC}}=+54.4 \mathrm{~km} \mathrm{~s}^{-1}, \sigma=84.4\right.$ $\left.\mathrm{km} \mathrm{s}{ }^{-1}\right)$. Similarly, our derived median galactocentric velocity for this field $\left(\mathrm{V}_{\mathrm{GC}}=+60.2 \mathrm{~km} \mathrm{~s}^{-1}\right)$ is in reasonably good agreement with the Shen et al. (2010) median galactocentric velocity model value of $\mathrm{V}_{\mathrm{GC}}=+71.6 \mathrm{~km} \mathrm{~s}^{-1} \quad\left(\sigma=65.7 \mathrm{~km} \mathrm{~s}^{-1}\right.$; Z. Li \& J. Shen 2012; priv. comm.). As was mentioned in $\S 4.1$, it is possible that the $(+8.5,+9)$ sight line may be partially contaminated by one of the structures associated with the $\mathrm{X}$-shaped bulge (e.g., see Saito et al. 2011; their Figure $3)$. This could result in additional substructure being present in our empirical velocity distribution function. However, visual inspection of Figure 12 does not indicate strong evidence of any additional features. This is supported by the results of Shapiro-Wilk and Anderson-Darling normality tests, which return $\mathrm{p}$-values of 0.288 and 0.114 , and indicate insufficient evidence to reject the null hypothesis that the $(+8.5,+9)$ velocity distribution is normal.

In Figure 14 we show the heliocentric radial velocity and dispersion distributions as a function of $[\mathrm{Fe} / \mathrm{H}]$ for all fields. As can be clearly seen in Figure 14, all three fields exhibit nearly identical trends of a decreasing velocity dispersion with increasing $[\mathrm{Fe} / \mathrm{H}]$. This observed trend is consistent with both the red clump and giant data analyzed in other outer bulge $\left(|\mathrm{b}| \gtrsim 6^{\circ}\right)$ fields (e.g., Minitti 1996; Babusiaux et al. 2010; de Propris et al. 2011; Johnson et al. 2011; Uttenthaler et al. 2012), and contrasts with the minor-axis results found by Babusiaux et al. (2010; their Figure 6) at $b=-4^{\circ}$ (opposite trend) and $b=-6^{\circ}$ (no trend). While de Propris et al. (2011) also find a correlation between the mean radial velocity and $[\mathrm{Fe} / \mathrm{H}]$ for red clump stars, Babusiaux et al. (2010) and Uttenthaler et al. (2012) do not find significant evidence of mean velocity variations as a function of metallicity. Visual inspection of Figure 14 indicates that we also do not find significant variations in mean/median velocity as a function of $[\mathrm{Fe} / \mathrm{H}]$ with two 
exceptions: (1) the metal-poor $([\mathrm{Fe} / \mathrm{H}]<-$ 1) stars in all fields and (2) the intermediate metallicity $(-0.5 \lesssim[\mathrm{Fe} / \mathrm{H}] \lesssim 0)$ stars in the $(+8.5,+9)$ field.

When the data are partitioned into 0.5 $\operatorname{dex}[\mathrm{Fe} / \mathrm{H}]$ bins, the median radial velocities within each bin of the $(-5.5,-7)$ and $(-4,-9)$ fields vary by $<20 \mathrm{~km} \mathrm{~s}^{-1}$ (ignoring the $[\mathrm{Fe} / \mathrm{H}]<-1$ stars $)$. The same is also true in the $(+8.5,+9)$ field where the stars with $-1<[\mathrm{Fe} / \mathrm{H}]<-0.5$ and $[\mathrm{Fe} / \mathrm{H}]>0$ have median radial velocities of -24.6 and -28.4 $\mathrm{km} \mathrm{s}^{-1}$, respectively. However, the stars in this field with $-0.5<[\mathrm{Fe} / \mathrm{H}]<0$ exhibit a noticeable velocity increase to $+28.8 \mathrm{~km}$ $\mathrm{s}^{-1}$. Although there is evidence that the $\mathrm{X}$-shaped bulge structure traced by the double red clump may preferentially contain stars with $[\mathrm{Fe} / \mathrm{H}]>-0.5$ (Ness et al. 2012; Uttenthaler et al. 2012), it is not clear if the increase in median velocity near $[\mathrm{Fe} / \mathrm{H}] \sim-0.5$ for our RGB sample is due to contamination from the nearby $\mathrm{X}$-shape structure or is simply a statistical fluctuation.

For the metal-poor $([\mathrm{Fe} / \mathrm{H}]<-1)$ stars in the $(-5.5,-7),(-4,-9)$, and $(+8.5,+9)$ fields the median heliocentric velocities are: $+49.6(\sigma=119.2) \mathrm{km} \mathrm{s}^{-1},-70.8(\sigma=117.4)$ $\mathrm{km} \mathrm{s}^{-1}$, and $-114(\sigma=109.3) \mathrm{km} \mathrm{s}^{-1}$, respectively. This contrasts with the more homogeneous set of stars with $[\mathrm{Fe} / \mathrm{H}]>-1$, which have median velocities and dispersions of: $-32.5(\sigma=66.1) \mathrm{km} \mathrm{s}^{-1},-42.1$ $(\sigma=79.4) \mathrm{km} \mathrm{s}^{-1}$, and $+17.4(\sigma=76.1) \mathrm{km}$ $\mathrm{s}^{-1}$. However, the relatively small number of stars in the most metal-poor bin of each field makes it difficult to assess the significance of the larger median velocities and dispersion of these stars. Therefore, we instead attempt to determine how many of the stars with $[\mathrm{Fe} / \mathrm{H}]<-1$ might be outliers by using the interquartile range (IQR) of the more metal-rich $([\mathrm{Fe} / \mathrm{H}]>-1)$ stars in each field. We define moderate outliers as stars with velocities of $1.5-3.0 \times \mathrm{IQR}$ above/below the median and extreme outliers as stars having velocities $>3.0 \times \mathrm{IQR}$ above/below the median. While we did not find extreme outliers in any of the fields, $\sim 20-40 \%$ of the metal-poor stars are moderate outliers and may belong to the halo. The small percentage of stars with both low $[\mathrm{Fe} / \mathrm{H}]$ and high velocities is consistent with the halo contamination estimate given in $\S 4.1 .1$.

\section{3. $\quad \alpha$-Element Abundances}

While iron may be produced by both short lived Type II supernovae (SNe) and longer lived Type Ia SNe, the $\alpha$ elements are primarily produced only by massive stars. As a consequence, the $[\alpha / \mathrm{Fe}]$ ratio can be a useful diagnostic for determining the chemical enrichment history of a stellar population (e.g. Tinsley 1979; Matteucci \& Brocato 1990). In particular, the magnitude of the $[\alpha / \mathrm{Fe}]$ ratios and the metallicity at which the $[\alpha / \mathrm{Fe}]$ ratio begins to decline (presumably due to the onset of Type Ia contributions) yield information about important quantities such as a population's initial mass function (IMF) and star formation rate (e.g., see review by McWilliam 1997 and references therein). Additionally, the star-to-star dispersion of the measured $[\alpha / \mathrm{Fe}]$ ratios in a given metallicity range can provide insight regarding the homogeneity of the gas from which the observed stars formed. In order to help address these issues in the bulge, we have measured the $[\mathrm{O} / \mathrm{Fe}],[\mathrm{Si} / \mathrm{Fe}]$, and 
$[\mathrm{Ca} / \mathrm{Fe}]$ ratios for stars in all three of our bulge fields.

In Figures 15 17 we plot [O/Fe], [Si/Fe], and $[\mathrm{Ca} / \mathrm{Fe}]$ as a function of $[\mathrm{Fe} / \mathrm{H}]$, respectively. To first order, all of the $[\mathrm{X} / \mathrm{Fe}]$ ratios follow the same general trend of being enhanced by $\gtrsim+0.3$ dex at $[\mathrm{Fe} / \mathrm{H}] \lesssim-$ 0.5 and then declining monotonically with increasing $[\mathrm{Fe} / \mathrm{H}]$. However, despite all of the $\alpha$-elements sharing the same $[\mathrm{Fe} / \mathrm{H}]$ value at which a steeper decline in their $[\mathrm{X} / \mathrm{Fe}]$ ratios occurs, it is clear from Figures 15, 17 that each element exhibits a slightly different trend. In particular, we note: (1) the $[\mathrm{O} / \mathrm{Fe}]$ ratios are $\sim 0.1-0.3$ dex larger than either $[\mathrm{Si} / \mathrm{Fe}]$ or $[\mathrm{Ca} / \mathrm{Fe}]$ for a given $[\mathrm{Fe} / \mathrm{H}]$, (2) the decline in $[\mathrm{Ca} / \mathrm{Fe}]$ with increasing $[\mathrm{Fe} / \mathrm{H}]$ is more shallow than for $[\mathrm{O} / \mathrm{Fe}]$ and $[\mathrm{Si} / \mathrm{Fe}],(3)$ the $[\mathrm{Si} / \mathrm{Fe}]$ data appear to continuously decline at $[\mathrm{Fe} / \mathrm{H}]>0$ while the $[\mathrm{O} / \mathrm{Fe}]$ and $[\mathrm{Ca} / \mathrm{Fe}]$ distributions appear to flatten out, and (4) the difference between the median $[\mathrm{X} / \mathrm{Fe}]$ ratios for stars with $[\mathrm{Fe} / \mathrm{H}]<-0.5$ and $[\mathrm{Fe} / \mathrm{H}]>-0.5$ is marginally larger for $[\mathrm{O} / \mathrm{Fe}]$ than either $[\mathrm{Si} / \mathrm{Fe}]$ or $[\mathrm{Ca} / \mathrm{Fe}]$ (see also Table 6). This last point has been noted previously (e.g., McWilliam \& Rich 2004; Zoccali et al. 2006; Fulbright et al. 2007; Alves-Brito et al. 2010; Ryde et al. 2010), and may be related to mass loss in Wolf-Rayet stars, rapidly rotating massive metal-poor stars, or metal-poor binary systems (e.g., McWilliam et al. 2008; Cescutti et al. 2009; but see also Ryde et al. 2010 and Alves-Brito et al. 2010). Unfortunately, we did not measure C, N, or $\mathrm{Mg}$ in our spectra and cannot comment further on the likelihood of our measured $[\mathrm{O} / \mathrm{Fe}]$ abundances being affected by mass and/or metallicity-dependent reductions in the oxygen yield from massive stars.

Interestingly, the individual abundance trends are nearly identical in all three of our fields, an observation that is highlighted in the bottom panels of Figures 1517. The similarities between the different fields is also evident by examining Table 6 , which summarizes the median $[\mathrm{X} / \mathrm{Fe}]$ ratios and star-to-star dispersions for the metal-poor $([\mathrm{Fe} / \mathrm{H}]<-0.5)$ and metal-rich $([\mathrm{Fe} / \mathrm{H}]>-0.5)$ groups in each field. The dispersion value, listed as $\sigma$ in Table 6 , represents the scatter around a best-fit line through each subpopulation, and is likely a fair representation of the measurement error. On average, the scatter for $[\mathrm{O} / \mathrm{Fe}],[\mathrm{Si} / \mathrm{Fe}]$, and $[\mathrm{Ca} / \mathrm{Fe}]$ only increases by $+0.06,+0.02$, and +0.02 dex between the metal-poor and metal-rich populations. These data support previous observations (e.g., Gonzalez et al. 2011; Johnson et al. 2011; Rich et al. 2012; Uttenthaler et al. 2012) that the bulge was well-mixed across a large volume and that large-scale IMF and star formation history differences were either not present or were diluted by mixing.

In order to examine further the small differences between the various $\alpha$-element trends, we compare our abundances with three bulge chemical enrichment models in Figure 18. The models in Figure18 include the primary bulge model from Kobayashi et al. (2011; solid red line), a model assuming a flat IMF ( $\mathrm{x}=0.3$; dashed green line), and a model assuming a flat IMF with both outflow (to reduce the Fe production) and a larger binary fraction than the solar neighborhood (dotted blue line). We find that the individual $\alpha$-element abundance trends are fit reasonably well 
by the nominal Kobayashi et al. (2011) model that assumes a Kroupa (2008) IMF $(\mathrm{x}=1.3)$ for massive stars, rapid star formation (shorter than the solar neighborhood), and a short star formation duration $(\sim 3$ Gyr). On the other hand, the one-zone flat IMF model clearly fails to reproduce the observed decrease in the $[\mathrm{X} / \mathrm{Fe}]$ ratios with increasing $[\mathrm{Fe} / \mathrm{H}]$, unless gas outflow is included to decrease the Fe yield. From these data we can conclude that: (1) a flat IMF model that does not include outflow (or some other way to decrease Fe production) fails to match the universal decline in $[\alpha / \mathrm{Fe}]$ at $[\mathrm{Fe} / \mathrm{H}] \gtrsim-0.5$ observed in our bulge fields, (2) the data are consistent with a model in which a significant portion of the bulge formed rapidly $(<3$ Gyr) and with a majority of its chemical enrichment driven by massive stars, and (3) the variations in the $[\mathrm{X} / \mathrm{Fe}]$ versus $[\mathrm{Fe} / \mathrm{H}]$ trends between the individual $\alpha$-elements are consistent with our current understanding of massive star nucleosynthesis. Although the Kobayashi et al. (2011) model is able to provide a reasonable fit to our $[\mathrm{O} / \mathrm{Fe}]$ and $[\mathrm{Ca} / \mathrm{Fe}]$ data, we note that the model values for $[\mathrm{Si} / \mathrm{Fe}]$ have been artificially reduced by -0.2 dex to provide a consistent fit. It is not clear if the Si offset is due to problems related to the nucleosynthesis codes (e.g., reaction rates; treatments of convection), the abundance codes (e.g., NLTE/3D effects; model atmosphere deficiencies), or both.

\subsubsection{Comparison with Other Bulge Fields}

Several past studies have examined the behavior of various $\alpha$-elements in fields along both the minor and major axis of the bulge (McWilliam \& Rich 1994; Rich
\& Origlia 2005; Cunha \& Smith 2006; Zoccali et al. 2006; Fulbright et al. 2007; Lecureur et al. 2007; Rich et al. 2007b; Meléndez et al. 2008; Alves-Brito et al. 2010; Bensby et al. 2010a; Ryde et al. 2010; Bensby et al. 2011; Gonzalez et al. 2011; Hill et al. 2011; Johnson et al. 2011; Bensby et al. 2013; Ness et al. 2013; Rich et al. 2012; Uttenthaler et al. 2012). All of these studies tend to find that metalpoor $([\mathrm{Fe} / \mathrm{H}] \lesssim-0.5)$ stars universally show enhancements of at least +0.3 dex in their $[\alpha / \mathrm{Fe}]$ ratios, with the more metal-rich stars exhibiting lower $[\alpha / \mathrm{Fe}]$ ratios. Additionally, despite differing data quality, instruments, and measurement techniques, a general consensus has emerged indicating that the $[\alpha / \mathrm{Fe}]$ versus $[\mathrm{Fe} / \mathrm{H}]$ trends are essentially identical among all bulge fields and that this may be especially true among bulge stars with $[\mathrm{Fe} / \mathrm{H}] \lesssim-0.5$.

In Figure 19 we overplot our derived $[\mathrm{O} / \mathrm{Fe}],[\mathrm{Si} / \mathrm{Fe}]$, and $[\mathrm{Ca} / \mathrm{Fe}]$ abundances as a function of $[\mathrm{Fe} / \mathrm{H}]$ with those available in the literature. Except for small offsets $(\lesssim 0.1$ dex $)$ due to systematic differences in abundance scales, our data are generally in good agreement with those available in other bulge fields. The lone exception appears to be the [Si/Fe] abundances at $[\mathrm{Fe} / \mathrm{H}] \gtrsim 0$. In particular, our $[\mathrm{Si} / \mathrm{Fe}]$ ratios appear to decline more rapidly with increasing $[\mathrm{Fe} / \mathrm{H}]$ than those found in other fields. Unfortunately, the reason for this discrepancy is not immediately clear, but it could result from effects such as the high excitation potential of the Si I lines used here or the sensitivity of especially the $6155 \AA$ Si I line to $\mathrm{CN}$ contamination in cool, metal-rich giants (see Figure 6). We note that there do not appear to be 
any strong trends between $[\mathrm{Si} / \mathrm{Fe}]$ and $\mathrm{T}_{\text {eff }}$ or between abundances derived from the 6145 and $6155 \AA$ Si I lines. Alternatively, the data may reflect a true [Si/Fe] abundance difference between metal-rich stars on the minor/major bulge axes and in offaxis fields. However, this seems less likely given the agreement between our data and the literature for $[\mathrm{O} / \mathrm{Fe}]$ and $[\mathrm{Ca} / \mathrm{Fe}]$ at $[\mathrm{Fe} / \mathrm{H}]>0$. In any case, the majority of our data, including the $[\mathrm{Si} / \mathrm{Fe}]$ ratios at $[\mathrm{Fe} / \mathrm{H}]<0$, extend the findings of previous work (e.g., Gonzalez et al. 2011) indicating that there is no significant $\alpha$-element gradient present in the bulge.

\subsubsection{Comparison with the Galactic Halo and Disk}

As mentioned in $\S 1$, early comparisons of the bulge's chemical composition to the other major Galactic stellar populations (i.e., disk and halo) seemed to indicate that bulge stars may be uniquely enhanced in their $[\alpha / \mathrm{Fe}]$ ratios (Zoccali et al. 2006; Fulbright et al. 2007; Lecureur et al. 2007), but recent studies have instead found that the bulge may be chemically similar to the thick disk and possibly metal-rich tail of the halo (Prochaska et al. 2000; Meléndez et al. 2008; Bensby et al. 2010a,b; Ryde et al. 2010; Alves-Brito et al. 2010; Bensby et al. 2011; Gonzalez et al. 2011; Hill et al. 2011; Johnson et al. 2011). However, it is not yet clear if the chemical similarity, particularly between the bulge and thick disk, extends to other light and heavy elements as well (e.g., Fulbright et al. 2007; Lecureur et al. 2007; Alves-Brito et al. 2010; Johnson et al. 2012).

In Figures 20 22 we compare our derived $[\mathrm{O} / \mathrm{Fe}],[\mathrm{Si} / \mathrm{Fe}]$, and $[\mathrm{Ca} / \mathrm{Fe}]$ abun- dances to those available in the literature for both giants and dwarfs in the halo, thick disk, and thin disk 20. First examining $[\mathrm{O} / \mathrm{Fe}]$ for stars with $[\mathrm{Fe} / \mathrm{H}]<0$, Figure 20 shows that the thick disk and halo exhibit nearly identical abundance patterns with respect to the bulge, albeit with a smaller star-to-star scatter. Further inspection of Figure 20 also reveals that our most metal-poor bulge stars may have slightly enhanced $(\sim 0.1-0.2 \mathrm{dex})[\mathrm{O} / \mathrm{Fe}]$ ratios relative to similar metallicity halo and thick disk giants. However, we do not believe this offset is significant because: (1) previous differential analyses have found good agreement for $[\mathrm{O} / \mathrm{Fe}]$ between these populations (e.g., Meléndez et al. 2008; Alves-Brito et al. 2010), (2) 0.1 dex of the offset is due to a zero point difference between this work and Alves-Brito et al. (2010; the green crosses in Figure 201), and (3) the observed difference is within the error bars of our $[\mathrm{O} / \mathrm{Fe}]$ measurements. While the thin disk stars with $[\mathrm{Fe} / \mathrm{H}] \lesssim 0$ exhibit lower $[\mathrm{O} / \mathrm{Fe}]$ ratios than the bulge, the situation becomes less clear at higher $[\mathrm{Fe} / \mathrm{H}]$. For super-solar metallicities the $[\mathrm{O} / \mathrm{Fe}]$ abundance trends of the thin disk, thick disk, and bulge appear to merge and may become indistinguishable.

For the $[\mathrm{Si} / \mathrm{Fe}]$ versus $[\mathrm{Fe} / \mathrm{H}]$ distributions shown in Figure 21, we again find that the halo and thick disk, especially at

\footnotetext{
${ }^{20}$ As has been noted in past work (e.g., Alves-Brito et al. 2010), a direct comparison between abundance trends derived from giant and dwarf spectra may be contaminated by zero-point offsets that can reach at least the 0.1-0.2 dex level. Therefore, in Figures 20 22 we include both giant and dwarf data for comparison, but caution that apparent systematic abundance differences $\lesssim 0.1$ dex in the $[\alpha / \mathrm{Fe}]$ ratios may not be significant.
} 
$[\mathrm{Fe} / \mathrm{H}]<0$, are nearly identical to that of the bulge. However, much like the $[\mathrm{O} / \mathrm{Fe}]$ distributions, any similarities between the bulge and thick disk become difficult to assess at $[\mathrm{Fe} / \mathrm{H}]>0$. The number of thick disk giants in this metallicity regime for which [Si/Fe] has been measured is too small to draw any firm conclusions. In contrast, the [Si $/ \mathrm{Fe}]$ ratios of the thin disk stars with $[\mathrm{Fe} / \mathrm{H}] \lesssim 0$ are underabundant relative to the bulge, and neither the dwarf nor giant trends exhibit the continued decline in $[\mathrm{Si} / \mathrm{Fe}]$ at $[\mathrm{Fe} / \mathrm{H}]>0$ seen in both our data and possibly those of other bulge studies (see Figure 191).

While the halo $[\mathrm{Ca} / \mathrm{Fe}]$ abundances derived from both dwarfs and giants are in excellent agreement with our bulge values, the thick disk $[\mathrm{Ca} / \mathrm{Fe}]$ ratios for stars with $[\mathrm{Fe} / \mathrm{H}] \lesssim-0.3$ are $\sim 0.1$ dex lower. Similarly, the thin disk $[\mathrm{Ca} / \mathrm{Fe}]$ abundances for stars with $[\mathrm{Fe} / \mathrm{H}]<0$ are considerably lower than those found in the bulge. However, the $[\mathrm{Ca} / \mathrm{Fe}]$ abundance differences between the thin disk, thick disk, and bulge again become difficult to untangle at $[\mathrm{Fe} / \mathrm{H}]>0$ (see Figure 22). For the more metal-poor thick disk giant sample, we can partially reconcile the $[\mathrm{Ca} / \mathrm{Fe}]$ abundance difference by applying the $\sim 0.05$ dex zero point offset between our abundance scale and that used by Alves-Brito et al. (2010). The zero point correction also affects the thin disk and halo giant samples, but does not alter our conclusions regarding the chemical similarities between these populations.

In summary, we find in agreement with recent studies that the metal-poor component of the bulge $([\mathrm{Fe} / \mathrm{H}] \lesssim 0)$ and thick disk share indistinguishable $[\alpha / \mathrm{Fe}]$ abundance distributions, and that these simi- larities are independent of the $\alpha$-element analyzed. Additionally, we find that the most metal-poor $([\mathrm{Fe} / \mathrm{H}]<-1)$ stars in the bulge also exhibit very similar $[\alpha / \mathrm{Fe}]$ distributions to the more metal-rich stars in the halo. However, we note that this may not be surprising given that some of our metal-poor bulge stars could be halo interlopers (see §4.1.1). In contrast, the thin disk stars are generally underabundant in their $[\alpha / \mathrm{Fe}]$ ratios compared to bulge stars, at least for $[\mathrm{Fe} / \mathrm{H}]<0$. For the most metalrich stars in the bulge, the $[\mathrm{O} / \mathrm{Fe}]$ and $[\mathrm{Ca} / \mathrm{Fe}]$ data suggest that there may be significant chemical overlap with the thin disk and/or metal-rich tail of the thick disk. While our data and those from past studies indicate that the bulge and especially thick disk may have shared similar chemical enrichment histories, more work is needed to determine how these two populations are linked.

\section{SUMMARY}

We have determined $[\mathrm{Fe} / \mathrm{H}],[\mathrm{O} / \mathrm{Fe}]$, $[\mathrm{Si} / \mathrm{Fe}]$, and $[\mathrm{Ca} / \mathrm{Fe}]$ abundance ratios, as well as radial velocities, for 264 RGB stars in three off-axis bulge fields centered near $(1, \mathrm{~b})=(-5.5,-7), \quad(-4,-9)$, and $(+8.5,+9)$. The abundances were derived from moderate resolution $(\mathrm{R} \approx 18,000)$, high $\mathrm{S} / \mathrm{N}$ $\left(\sim 75-300\right.$ pixel $\left.^{-1}\right)$ spectra obtained with the Hydra multiobject spectrographs on the Blanco 4m and WIYN 3.5m telescopes. While the $[\mathrm{Fe} / \mathrm{H}]$ abundances were measured using equivalent width analyses, the $[\mathrm{O} / \mathrm{Fe}],[\mathrm{Si} / \mathrm{Fe}]$, and $[\mathrm{Ca} / \mathrm{Fe}]$ abundances were measured using spectrum synthesis techniques. The stars were selected from the blue side of the giant branch, but an analysis of the color-metallicity relation in 
other bulge samples that span a broader color range suggests this may not introduce a strong metallicity bias.

The metallicity distribution functions for all three fields are in general agreement with those found in minor-axis, outer bulge fields. In particular, the full range in $[\mathrm{Fe} / \mathrm{H}]$ found in each field spans from roughly $[\mathrm{Fe} / \mathrm{H}]=-1.5$ to +0.5 dex, and the median metallicity for the two southern bulge fields decreases with increasing Galactic latitude. Combining our $(-5.5,-7)$ and $(-4,-9)$ metallicity distributions with the Gonzalez et al. (2011) data at (+5.25,-3 ) indicates that the vertical metallicity gradient observed by Zoccali et al. (2008) along the minor-axis is also present in offaxis fields. We find a similar $[\mathrm{Fe} / \mathrm{H}]$ gradient of $\sim 0.4$ dex kpc ${ }^{-1}$. However, there does not seem to be a similarly strong radial $[\mathrm{Fe} / \mathrm{H}]$ gradient. Interestingly, the northern bulge field at $(+8.5,+9)$ was found to have the highest median metallicity $([\mathrm{Fe} / \mathrm{H}]=-0.23)$ sample. However, it is not clear if this is a selection effect resulting from redder stars being observed in this field, an indication of asymmetry in the metallicity distribution of the bulge, or possible contamination in the field by the $\mathrm{X}$-shaped (and possibly metal-rich) bulge structure.

The radial velocities and dispersion derived here are in good agreement with the BRAVA survey, and by extension are in reasonable agreement with the Shen et al. (2010) model. All three of our fields are also found to exhibit nearly identical radial velocity dispersion versus $[\mathrm{Fe} / \mathrm{H}]$ relations that show a strong decrease in dispersion with increasing metallicity. This trend matches previous observations of outer bulge fields, but contrasts with the dispersion-metallicity relation found at $|\mathrm{b}|<6^{\circ}$. In the two southern bulge fields, we do not find any significant variations with respect to the median velocity as a function of $[\mathrm{Fe} / \mathrm{H}]$. The same is also true for the more metal-poor and metal-rich stars in the northern field, but there is an unexpected increase in median velocity for stars with $-0.5<[\mathrm{Fe} / \mathrm{H}]<0$ in this field. It is possible that this effect could be tied to the $\mathrm{X}$-shaped bulge structure that resides near the $(+8.5,+9)$ sight line.

We find in agreement with past studies that the $[\alpha / \mathrm{Fe}]$ ratios are enhanced in all bulge fields at $[\mathrm{Fe} / \mathrm{H}] \lesssim-0.5$, but the $\alpha$-element abundances monotonically decline at higher metallicities. Additionally, the $[\mathrm{O} / \mathrm{Fe}],[\mathrm{Si} / \mathrm{Fe}]$, and $[\mathrm{Ca} / \mathrm{Fe}]$ abundance trends exhibit remarkable homogeneity across all fields analyzed in this work and previous studies. A significant $[\alpha / \mathrm{Fe}]$ abundance gradient does not appear to exist in the bulge. Small variations in the slope and magnitude of the individual $\alpha$-element trends are consistent with our current understanding of massive star nucleosynthesis, and support the idea that the bulge was well-mixed and formed more rapidly than the local thin disk. However, we find in agreement with several recent studies that for $[\mathrm{Fe} / \mathrm{H}] \lesssim 0$ the bulge $[\alpha / \mathrm{Fe}]$ ratios are indistinguishable from the halo and thick disk. Unfortunately, it is not yet clear if the similar $[\alpha / \mathrm{Fe}]$ trends between especially the bulge and thick disk extend to super-solar metallicities.

This research has made use of NASA's Astrophysics Data System Bibliographic Services. This material is based upon work 
supported by the National Science Foundation under award No. AST-1003201 to CIJ. CAP gratefully acknowledges support from the Daniel Kirkwood Research Fund at Indiana University. RMR acknowledges support from NSF grant AST-0709479 and AST-121120995. AK thanks the Deutsche Forschungsgemeinschaft for funding from Emmy-Noether grant Ko 4161/1.

\section{REFERENCES}

Alonso, A., Arribas, S., \& Martínez-Roger, C. 1999, A\&AS, 140, 261

Alonso, A., Arribas, S., \& Martínez-Roger, C. 2001, A\&A, 376, 1039

Alves-Brito, A., Meléndez, J., Asplund, M., Ramírez, I., \& Yong, D. 2010, A\&A, 513, A35

Babusiaux, C., Gómez, A., Hill, V., et al. 2010, A\&A, 519, A77

Bensby, T., Feltzing, S., \& Lundström, I. 2003, A\&A, 410, 527

Bensby, T., Feltzing, S., Lundström, I., \& Ilyin, I. 2005, A\&A, 433, 185

Bensby, T., Feltzing, S., Johnson, J. A., et al. 2010a, A\&A, 512, A41

Bensby, T., Alves-Brito, A., Oey, M. S., Yong, D., \& Meléndez, J. 2010b, A\&A, 516, L13

Bensby, T., Adén, D., Meléndez, J., et al. 2011, A\&A, 533, A134

Bensby, T., Yee, J. C., Feltzing, S., et al. 2013, A\&A, 549, A147

Bershady, M., Barden, S., Blanche, P.-A., et al. 2008, Proc. SPIE, 7014, 15
Brewer, M.-M., \& Carney, B. W. 2006, AJ, 131,431

Castelli, F., Gratton, R. G., \& Kurucz, R. L. 1997, A\&A, 318, 841

Cescutti, G., Matteucci, F., McWilliam, A., \& Chiappini, C. 2009, A\&A, 505, 605

Clarkson, W., Sahu, K., Anderson, J., et al. 2008, ApJ, 684, 1110

Cunha, K., \& Smith, V. V. 2006, ApJ, 651, 491

De Propris, R., Rich, R. M., Kunder, A., et al. 2011, ApJ, 732, L36

Dotter, A., Chaboyer, B., Jevremović, D., et al. 2007, AJ, 134, 376

Edvardsson, B., Andersen, J., Gustafsson, B., et al. 1993, A\&A, 275, 101

Fiorucci, M., \& Munari, U. 2003, A\&A, 401,781

Fulbright, J. P. 2000, AJ, 120, 1841

Fulbright, J. P., McWilliam, A., \& Rich, R. M. 2006, ApJ, 636, 821

Fulbright, J. P., McWilliam, A., \& Rich, R. M. 2007, ApJ, 661, 1152

Girard, T. M., van Altena, W. F., Zacharias, N., et al. 2011, AJ, 142, 15

González Hernández, J. I., \& Bonifacio, P. 2009, A\&A, 497, 497

Gonzalez, O. A., Rejkuba, M., Zoccali, M., et al. 2011, A\&A, 530, A54 
Hinkle, K., Wallace, L., Valenti, J., \& Harmer, D. 2000, Visible and Near Infrared Atlas of the Arcturus Spectrum 3727-9300 A ed. Kenneth Hinkle, Lloyd Wallace, Jeff Valenti, and Dianne Harmer. (San Francisco: ASP) ISBN: 158381-037-4, 2000

Howard, C. D., Rich, R. M., Reitzel, D. B., et al. 2008, ApJ, 688, 1060

Johnson, J. A. 2002, ApJS, 139, 219

Johnson, C. I., Pilachowski, C. A., Simmerer, J., \& Schwenk, D. 2008, ApJ, 681,1505

Johnson, C. I., Rich, R. M., Fulbright, J. P., Valenti, E., \& McWilliam, A. 2011, ApJ, 732, 108

Johnson, C. I., Rich, R. M., Kobayashi, C., \& Fulbright, J. P. 2012, ApJ, 749, 175

Knezek, P. M., Bershady, M. A., Willmarth, D., et al. 2010, Proc. SPIE, 7735, 240

Kobayashi, C., Karakas, A. I., \& Umeda, H. 2011, MNRAS, 414, 3231

Kroupa, P. 2008, Pathways Through an Eclectic Universe, 390, 3

Kunder, A., Koch, A., Rich, R. M., et al. 2012, AJ, 143, 57

Kučinskas, A., Hauschildt, P. H., Ludwig, H.-G., et al. 2005, A\&A, 442, 281

Kučinskas, A., Hauschildt, P. H., Brott, I., et al. 2006, A\&A, 452, 1021

Kupka, F. G., Ryabchikova, T. A., Piskunov, N. E., Stempels, H. C., \& Weiss, W. W. 2000, Baltic Astronomy, 9,590
Lecureur, A., Hill, V., Zoccali, M., et al. 2007, A\&A, 465, 799

Matteucci, F., \& Brocato, E. 1990, ApJ, 365,539

McWilliam, A. 1997, ARA\&A, 35, 503

McWilliam, A., \& Rich, R. M. 1994, ApJS, 91, 749

McWilliam, A., \& Rich, R. M. 2004, Origin and Evolution of the Elements, ed. A. McWilliam \& M. Rausch (Pasadena, CA: Carnegie Obs.)

McWilliam, A., \& Zoccali, M. 2010, ApJ, 724,1491

McWilliam, A., Matteucci, F., Ballero, S., et al. 2008, AJ, 136, 367

McWilliam, A., Fulbright, J., \& Rich, R. M. 2010, IAU Symposium, 265, 279

Meléndez, J., Asplund, M., Alves-Brito, A., et al. 2008, A\&A, 484, L21

Minniti, D. 1996, ApJ, 459, 579

Moultaka, J., Ilovaisky, S. A., Prugniel, P., \& Soubiran, C. 2004, PASP, 116, 69

Ness, M., Freeman, K., Athanassoula, E., et al. 2012, ApJ, 756, 22

Ness, M., Freeman, K., Athanassoula, E., et al. 2013, MNRAS, 635

Nissen, P. E., \& Schuster, W. J. 1997, A\&A, 326, 75

Peterson, R. C., Dalle Ore, C. M., \& Kurucz, R. L. 1993, ApJ, 404, 333 
Press, W. H., Teukolsky, S. A., Vetterling, W. T., \& Flannery, B. P. 1992, Cambridge: University Press, —c1992, 2nd ed.

Prochaska, J. X., Naumov, S. O., Carney, B. W., McWilliam, A., \& Wolfe, A. M. 2000, AJ, 120, 2513

Ralchenko, Yu., Kramida, A.E., Reader, J., and NIST ASD Team (2011).NIST Atomic Spectra Database (version 4.1). National Institute of Standards and Technology, Gaithersburg, MD.

Ramírez, I., \& Allende Prieto, C. 2011, ApJ, 743, 135

Reddy, B. E., Tomkin, J., Lambert, D. L., \& Allende Prieto, C. 2003, MNRAS, 340, 304

Reddy, B. E., Lambert, D. L., \& Allende Prieto, C. 2006, MNRAS, 367, 1329

Rich, R. M. 1990, ApJ, 362, 604

Rich, R. M., \& Origlia, L. 2005, ApJ, 634, 1293

Rich, R. M., Reitzel, D. B., Howard, C. D., \& Zhao, H. 2007a, ApJ, 658, L29

Rich, R. M., Origlia, L., \& Valenti, E. 2007b, ApJ, 665, L119

Rich, R. M., Origlia, L., \& Valenti, E. 2012, ApJ, 746, 59

Robin, A. C., Reylé, C., Derrière, S., \& Picaud, S. 2003, A\&A, 409, 523

Ryde, N., Gustafsson, B., Edvardsson, B., et al. 2010, A\&A, 509, A20
Saha, K., Martinez-Valpuesta, I., \& Gerhard, O. 2012, MNRAS, 421, 333

Saito, R. K., Zoccali, M., McWilliam, A., et al. 2011, AJ, 142, 76

Schlegel, D. J., Finkbeiner, D. P., \& Davis, M. 1998, ApJ, 500, 525

Shen, J., Rich, R. M., Kormendy, J., et al. 2010, ApJ, 720, L72

Skrutskie, M. F., Cutri, R. M., Stiening, R., et al. 2006, AJ, 131, 1163

Sneden, C. 1973, ApJ, 184, 839

Soto, M., Rich, R. M., \& Kuijken, K. 2007, ApJ, 665, L31

Stephens, A., \& Boesgaard, A. M. 2002, AJ, 123, 1647

Thevenin, F. 1990, A\&AS, 82, 179

Tinsley, B. M. 1979, ApJ, 229, 1046

Tomkin, J., Lemke, M., Lambert, D. L., \& Sneden, C. 1992, AJ, 104, 1568

Uttenthaler, S., Schultheis, M., Nataf, D. M., et al. 2012, A\&A, 546, A57

Zoccali, M., Lecureur, A., Barbuy, B., et al. 2006, A\&A, 457, L1

Zoccali, M., Hill, V., Lecureur, A., et al. 2008, A\&A, 486, 177

This 2-column preprint was prepared with the AAS LATEX macros v5.2. 


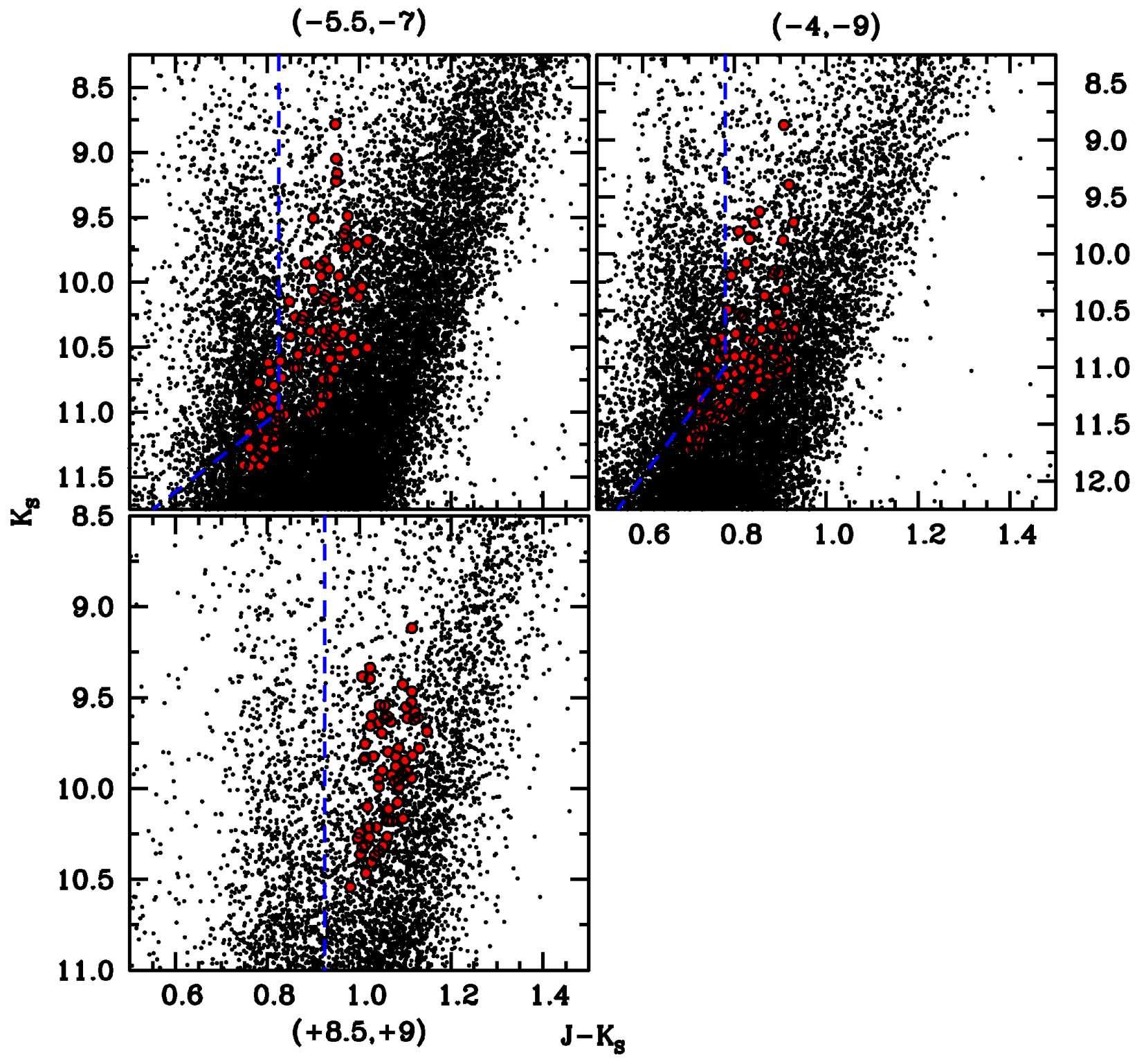

Fig. 1. - $\mathrm{K}_{\mathrm{S}}$ versus $\mathrm{J}-\mathrm{K}_{\mathrm{S}}$ color-magnitude diagrams for the three observed bulge fields. The small black circles are point sources from the 2MASS catalog, and the filled red circles are the stars observed in the $(1, b)=\left(-5.5^{\circ},-7^{\circ}\right),\left(-4^{\circ},-9^{\circ}\right)$, and $\left(+8.5^{\circ},+9^{\circ}\right)$ fields. The dashed blue lines designate the approximate separation between the bulge RGB and the foreground red clump population. 


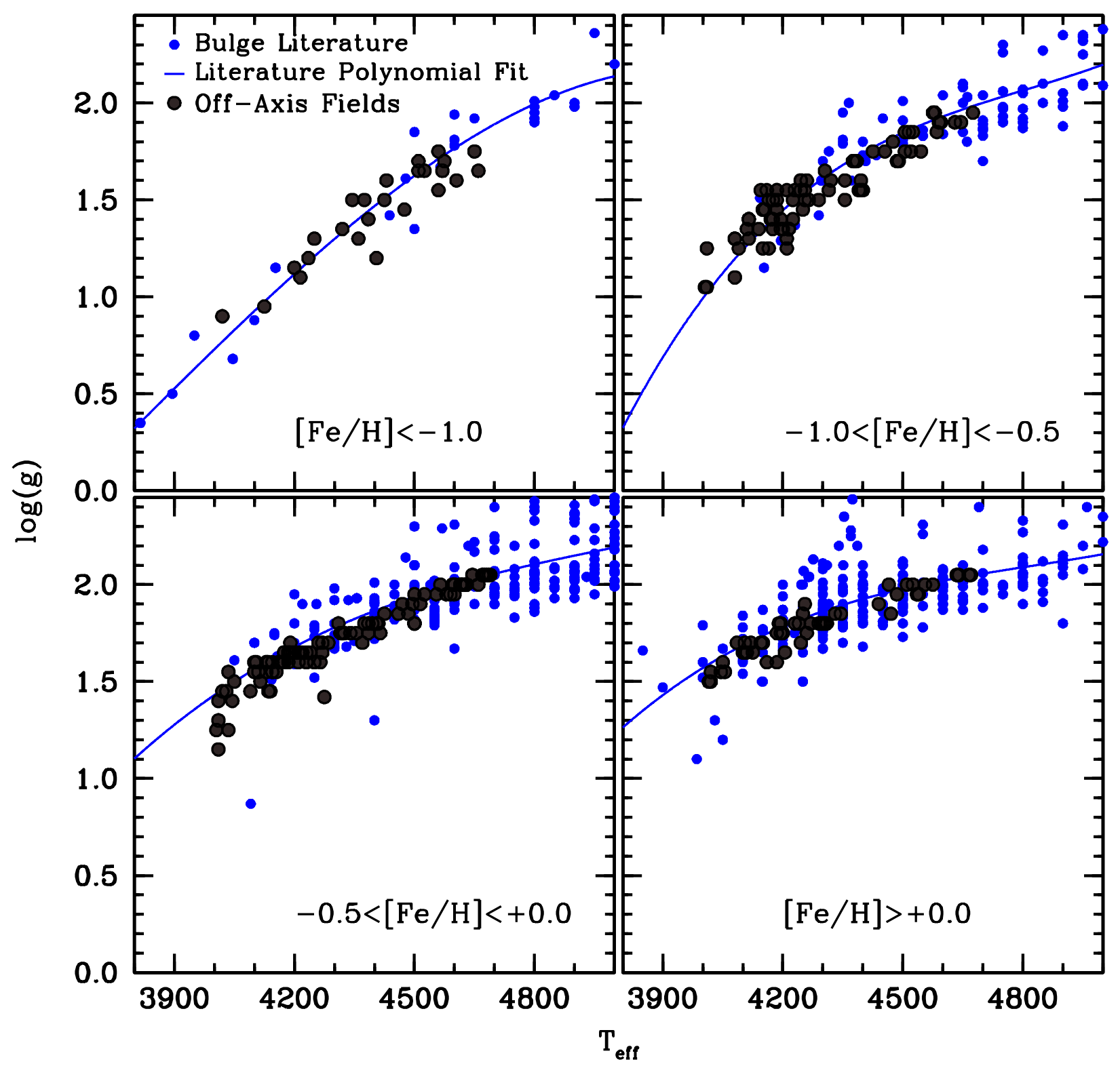

Fig. 2.- The four panels show the relationship between $\log (\mathrm{g})$ and $\mathrm{T}_{\text {eff }}$ for bulge RGB stars in our combined sample (filled grey circles) and those available in the literature (filled blue circles). Each panel contains only stars within the listed metallicity range. A $3^{\text {rd }}$ order polynomial is fit to the literature data and is illustrated by the solid blue lines. The literature data are from: McWilliam \& Rich (1994), Rich \& Origlia (2005), Fulbright et al. (2006), Lecureur et al. (2007), Rich et al. (2007b), Meléndez et al. (2008), Zoccali et al. (2008), Alves-Brito et al. (2010), Ryde et al. (2010), Johnson et al. (2011), and Rich et al. (2012). 


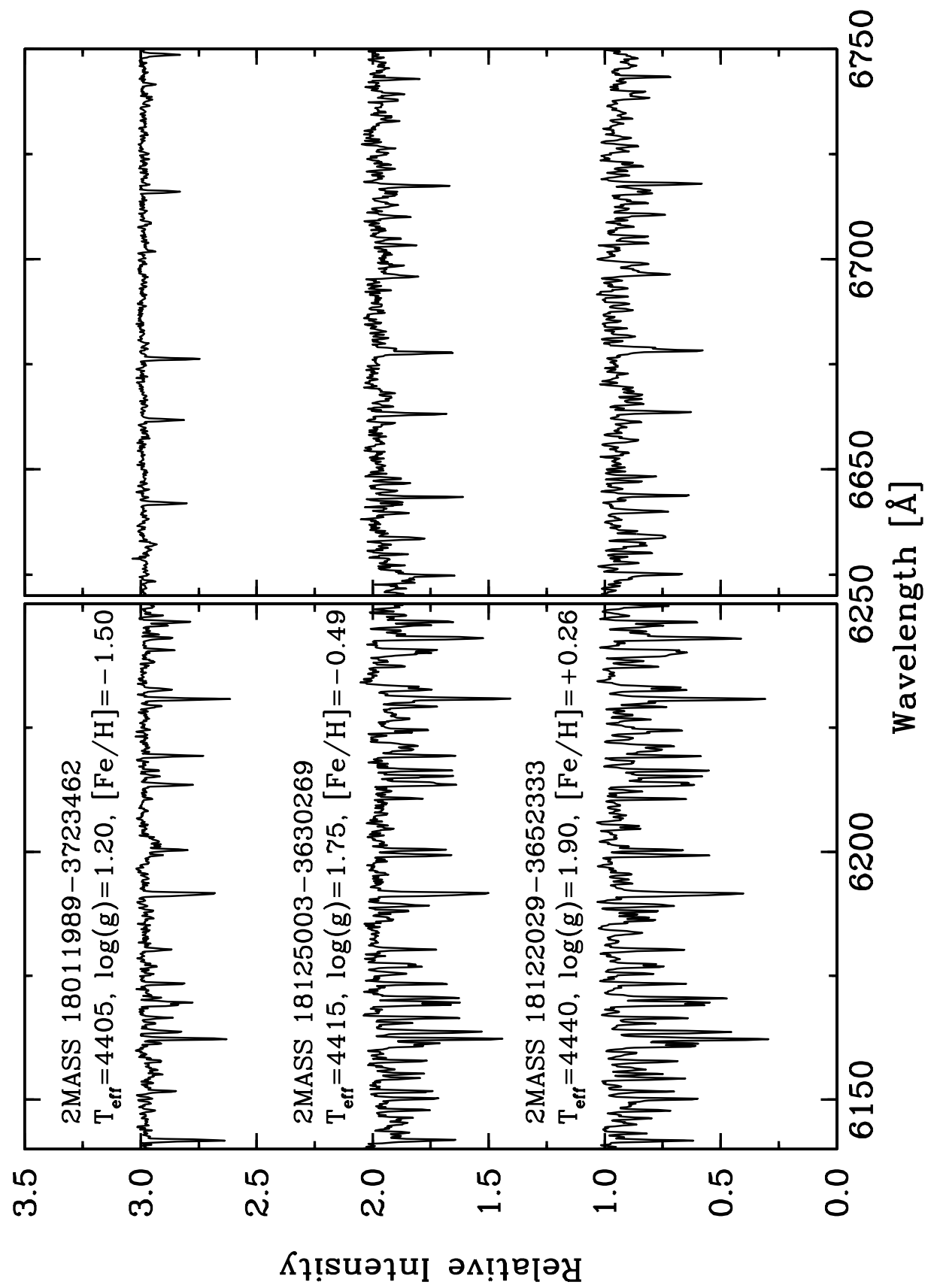

Fig. 3.- Typical sample spectra for stars of comparable $\mathrm{T}_{\text {eff }}$ are shown to illustrate how the continuum windows, line strengths, and molecular features change as a function of metallicity. The left panel shows a portion of the $6300 \AA$ region spectra, and the right panel shows a portion of the $6700 \AA$ region spectra for the same three stars. The spectra have been offset for display purposes. 


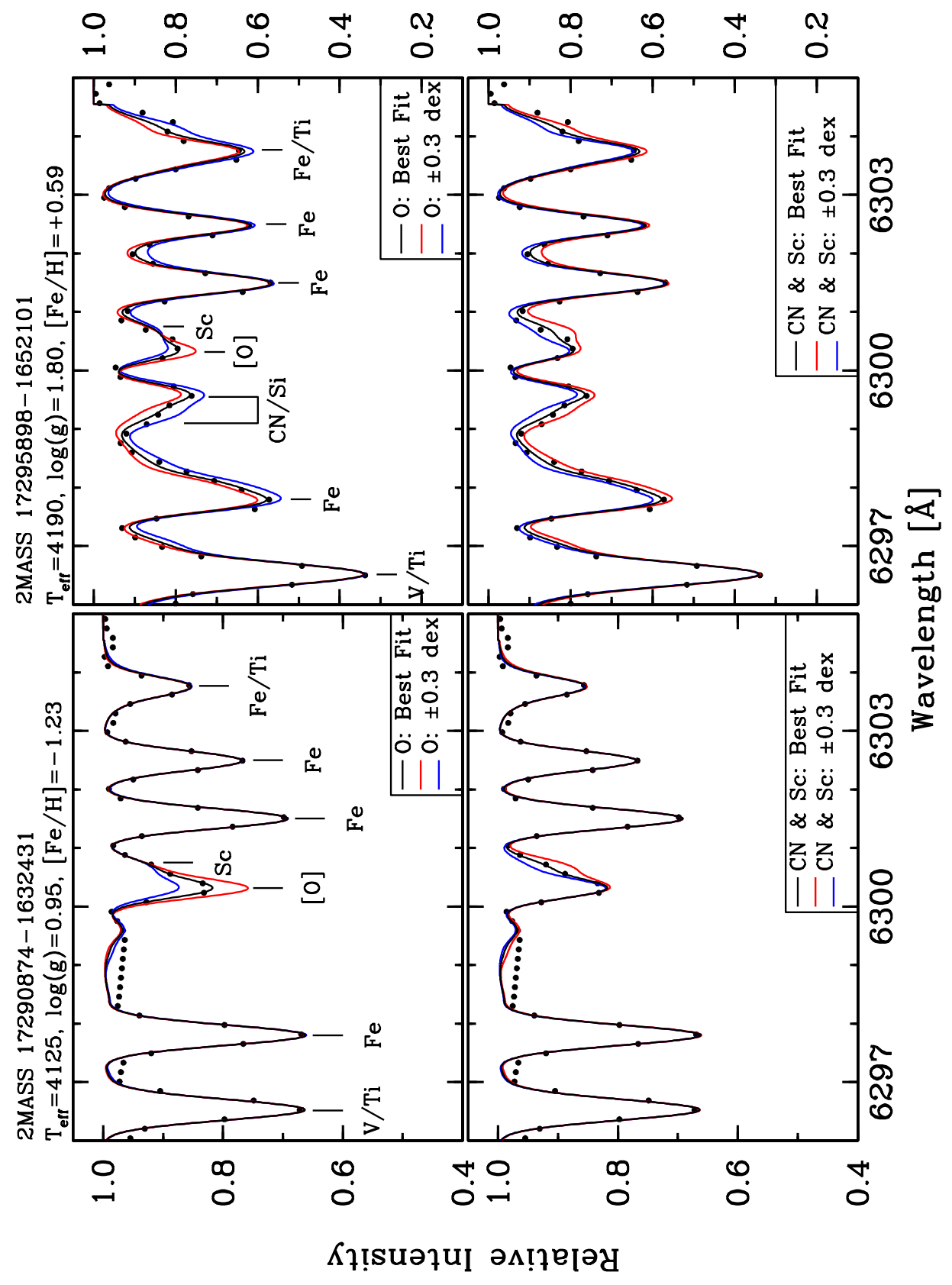

Fig. 4.- Sample spectrum synthesis fits to the region around the $6300 \AA$ [O I] line for a cool, metal-poor star (left panels) and a cool metal-rich star (right panels). In the top left and top right panels, the solid black line shows the best fit oxygen abundance. The colored solid lines illustrate how the syntheses change when the oxygen abundance is altered by +0.3 dex (red lines) and -0.3 dex (blue lines) from the best-fit value. Similarly, the bottom left and bottom right panels show how the syntheses change when oxygen is held fixed at the best-fit value while the $\mathrm{CN}$ and $\mathrm{Sc}$ abundances are altered by \pm 0.3 dex. 


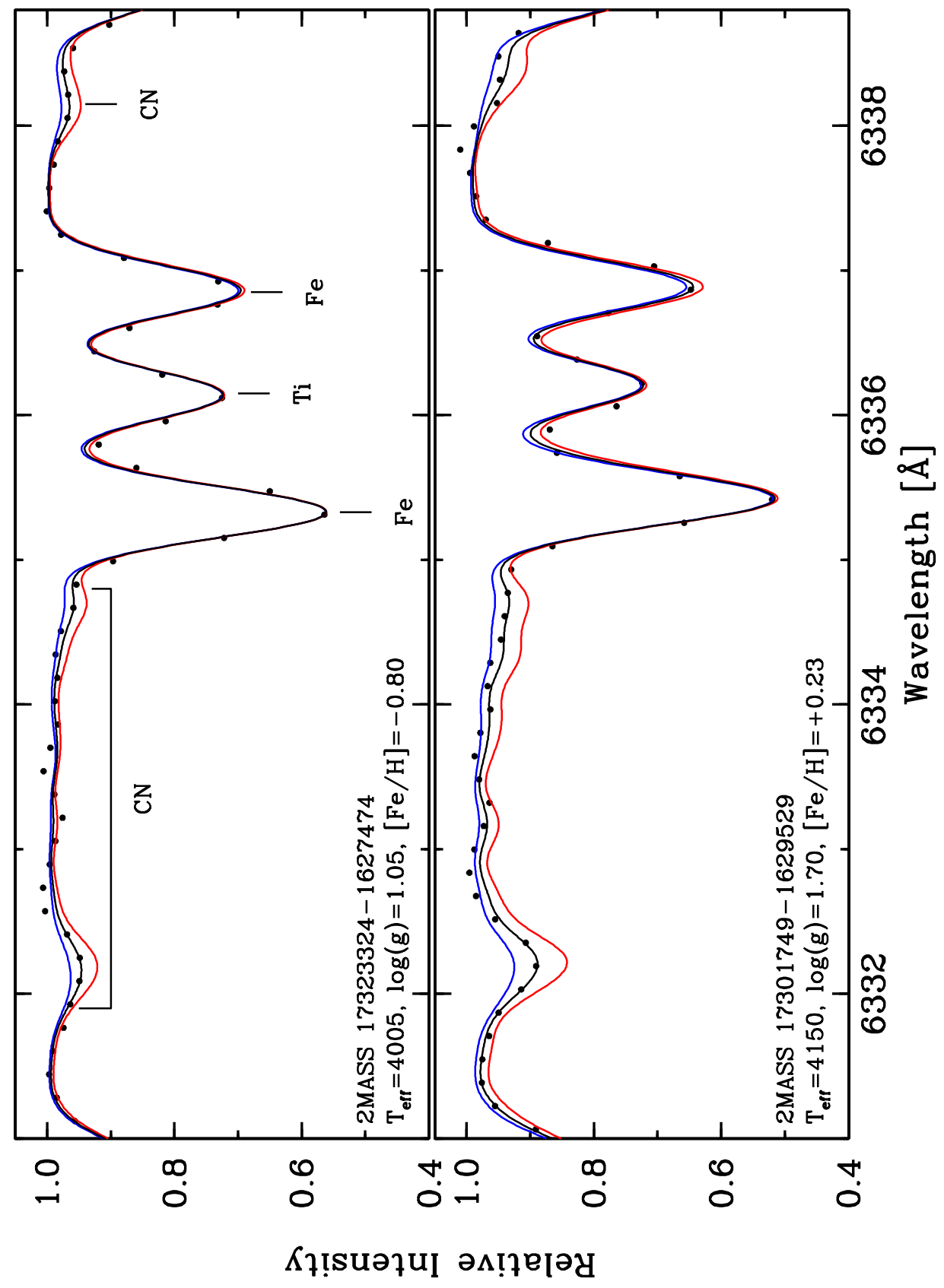

Fig. 5.- Similar to Figure 4, the synthetic spectrum fits shown here illustrate how the CN abundance was determined for each star. The top panel shows a cool, metal-poor example and the bottom panel shows a cool, metal-rich example. The black line indicates the best fit $\mathrm{CN}$ abundance. The red and blue syntheses indicate changes in the CN abundance by \pm 0.3 dex, respectively. Note that except for a weak Si I and Fe II blend near $6332 \AA$, the two regions spanning 6331.5-6335 $\AA$ and $6337.5-6339.8 \AA$ contain almost exclusively $\mathrm{CN}$ features. 


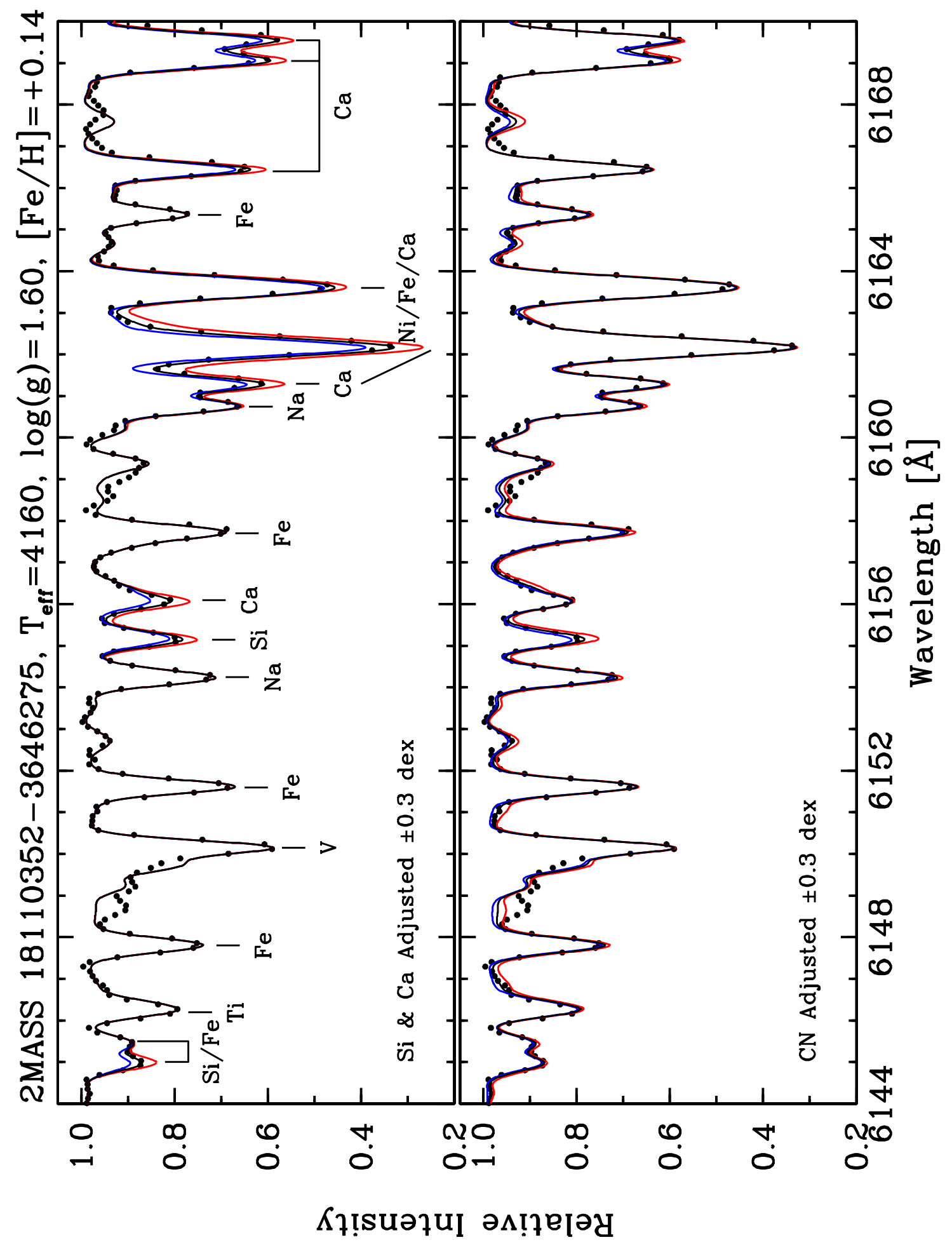

Fig. 6.- Similar to Figure 4, the synthetic spectrum fits shown here illustrate how the $\mathrm{Si}$ and $\mathrm{Ca}$ line profiles change when the $\mathrm{Si}$ and $\mathrm{Ca}$ abundances are altered \pm 0.3 dex from the best-fit value (top panel) and when the $\mathrm{CN}$ abundance is altered by \pm 0.3 dex from the best-fit value (bottom panel). Note the particular sensitivity of the $6155 \mathrm{Si}$ I line to changes in the $\mathrm{CN}$ abundance. 


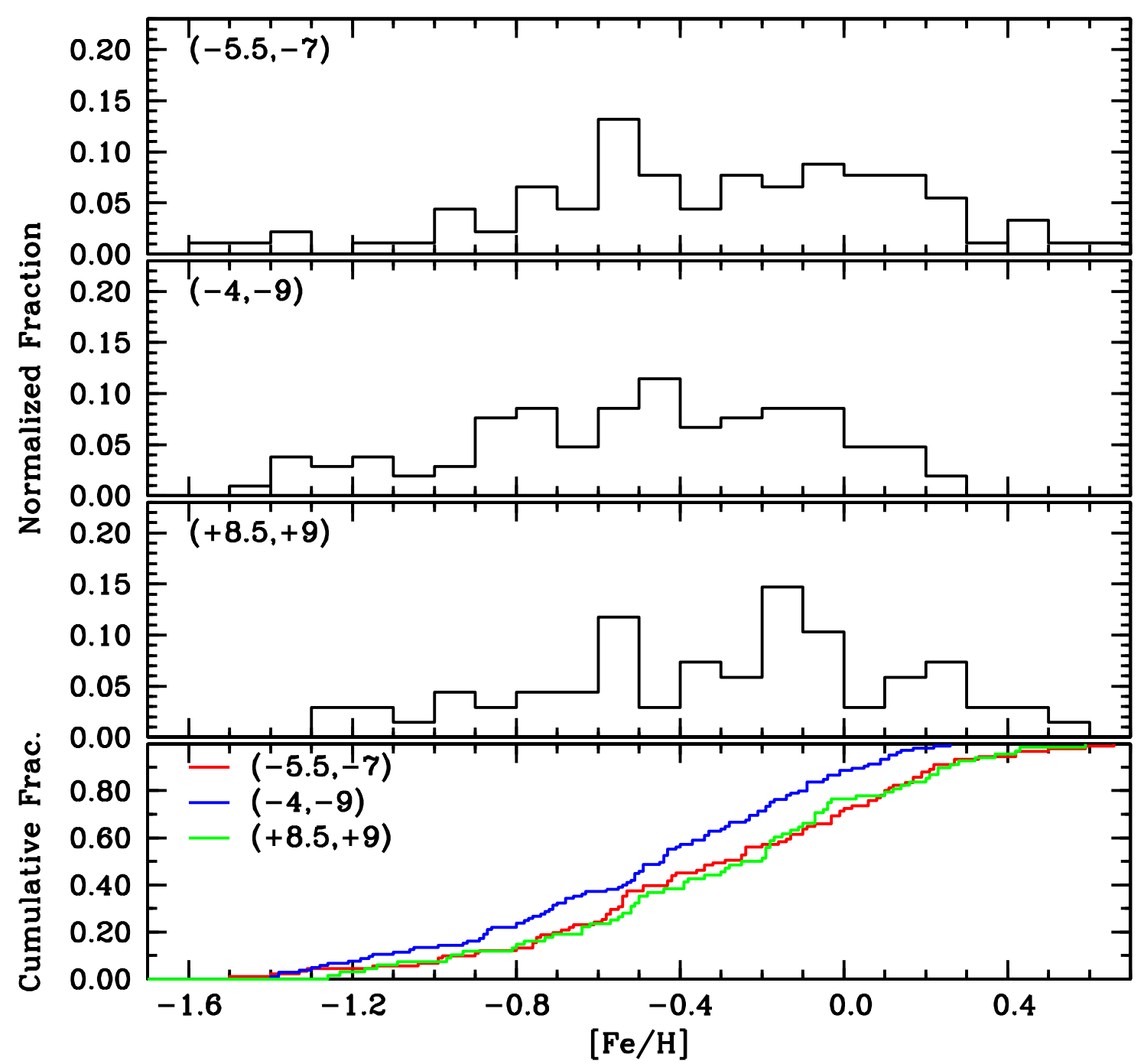

Fig. 7.- The top three panels show the metallicity distribution functions for all three bulge fields binned in 0.1 dex increments. The bottom panel compares the cumulative distribution functions for all three fields. 


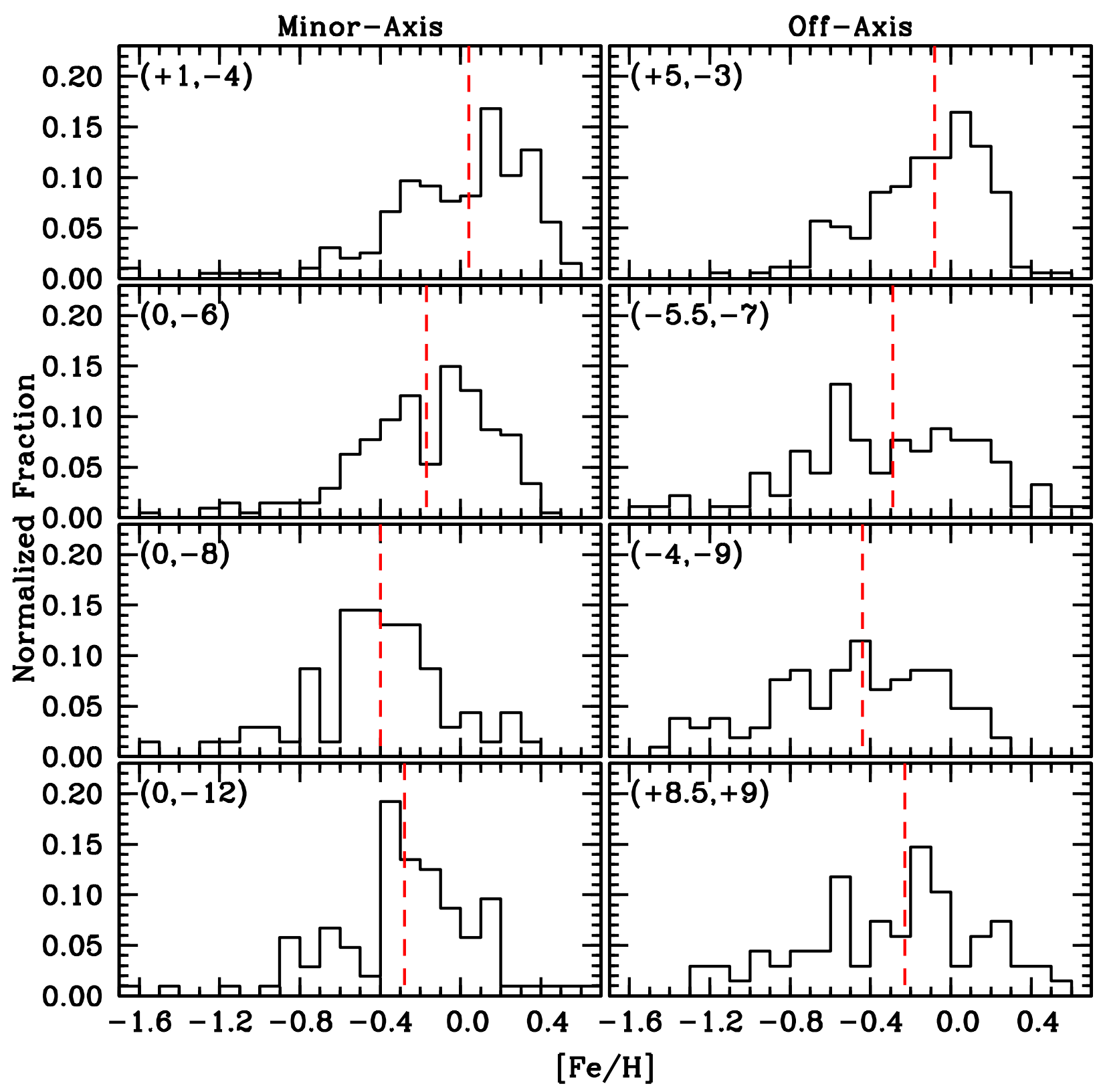

Fig. 8.- Metallicity distribution functions are shown for bulge RGB stars in four minoraxis (left panels) and four off-axis (right panels) fields. The dashed red line in each panel indicates the median $[\mathrm{Fe} / \mathrm{H}]$ value. The $(\mathrm{l}, \mathrm{b})=(+1,-4),(0,-6)$, and $(0,-12)$ fields are from Zoccali et al. (2008), the $(0,-8)$ field is from Johnson et al. (2011), the $(+5,-3)$ field is from Gonzalez et al. (2011), and the $(-5.5,-7),(-4,-9)$, and $(+8.5,+9)$ fields are from the present work. Note that the decreasing metallicity gradient with increasing Galactic latitude appears to be present along both the minor axis and off-axis. 


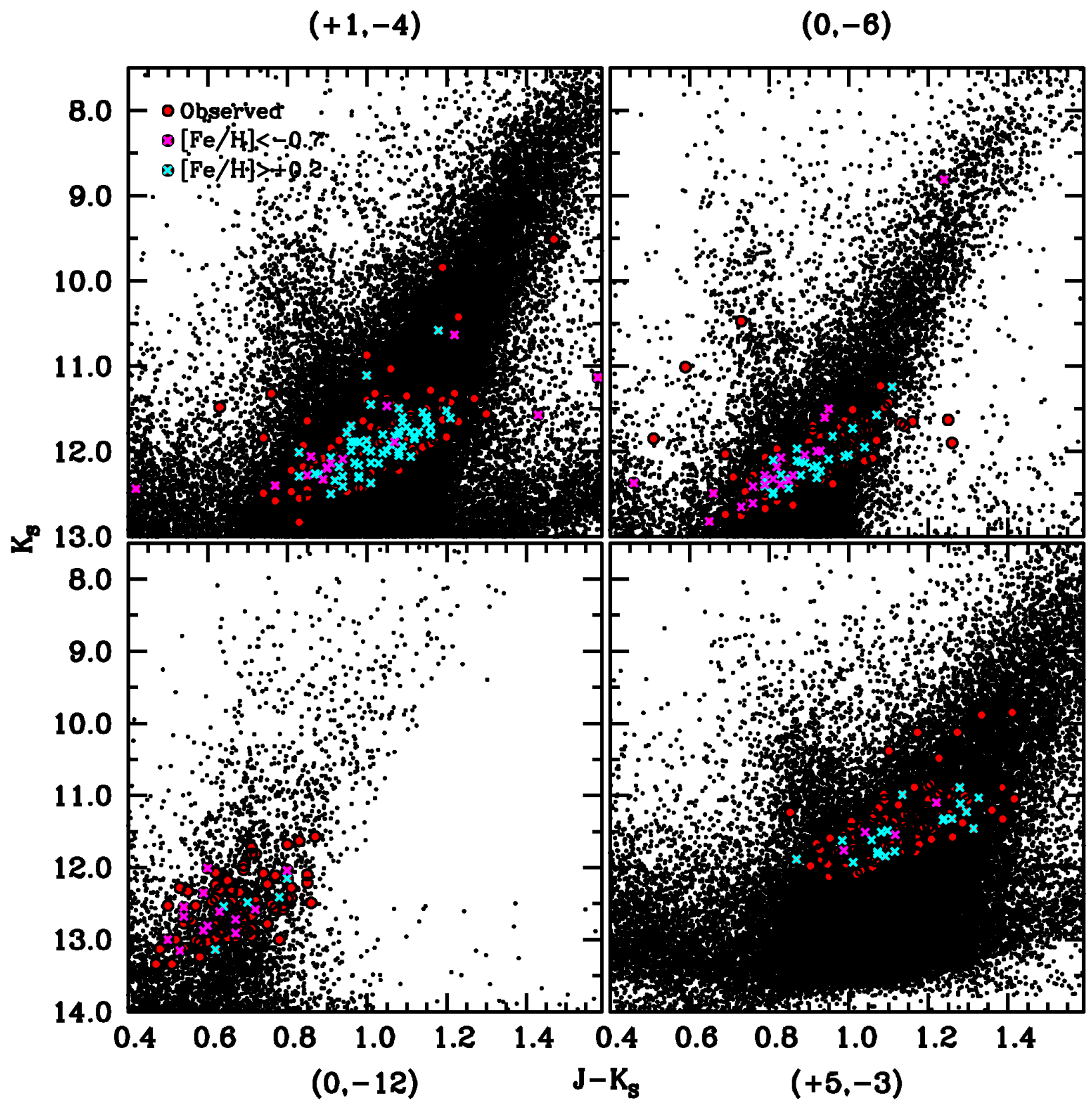

Fig. 9.- Color-magnitude diagrams using 2MASS data (small black points) are shown for the bulge fields presented in Zoccali et al. (2008) and Gonzalez et al. (2011). The filled red circles are the observed stars in each field, those with magenta crosses are the most metal-poor stars $([\mathrm{Fe} / \mathrm{H}]<-0.7)$, and those with cyan crosses are the most metal-rich stars $([\mathrm{Fe} / \mathrm{H}]>+0.2)$. Note that both metal-poor and metal-rich stars are found over a broad range in $\mathrm{J}-\mathrm{K}_{\mathrm{S}}$ color. 


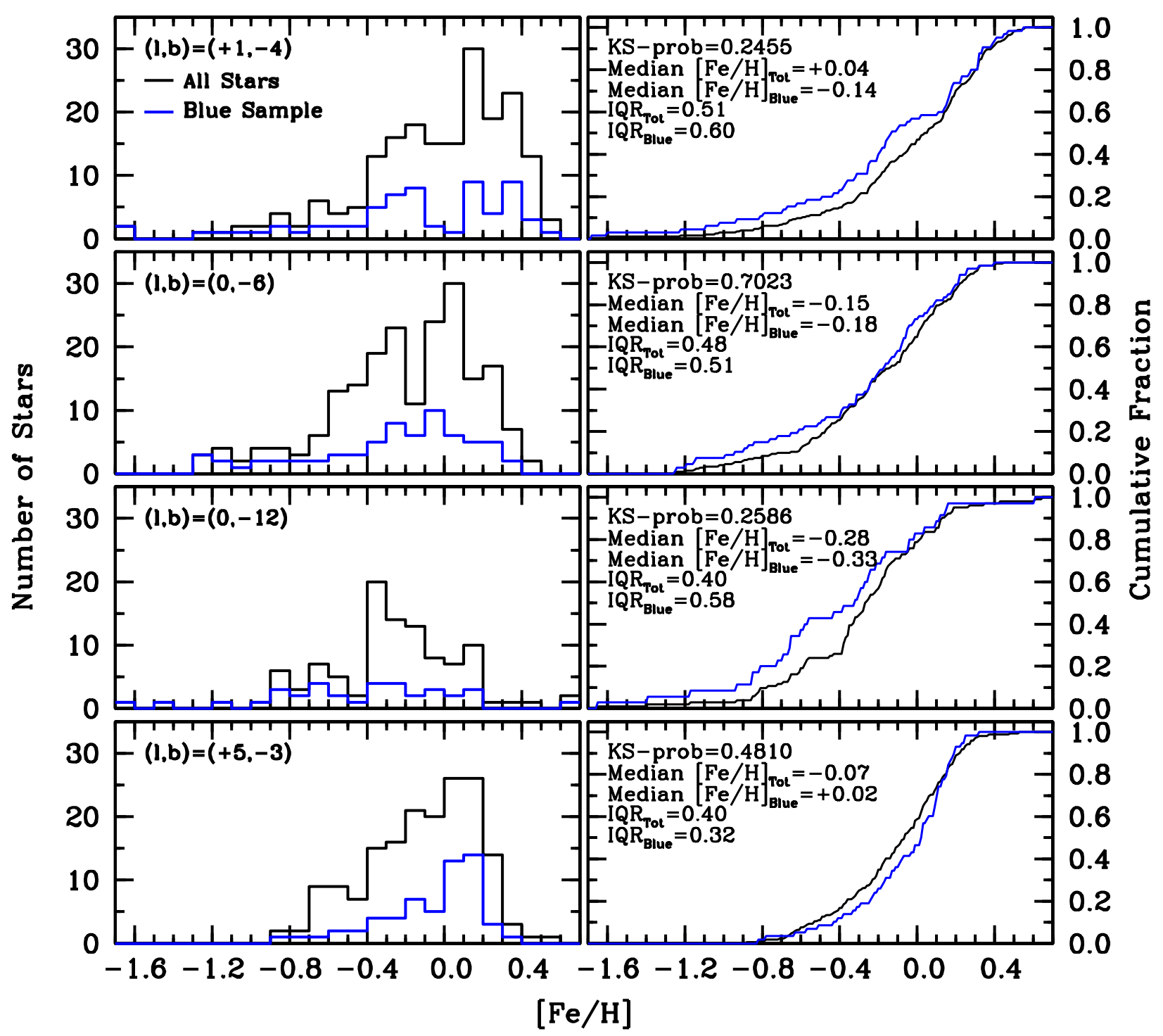

Fig. 10.- Metallicity distribution histograms and cumulative distribution functions are shown for the full samples (solid black lines) presented in Zoccali et al. (2008) and Gonzalez et al. (2011). Similar data are plotted using just the bluest third of each sample (solid blue lines), which roughly matches our sample selection. In the right panels we include the results of two-sided KS tests (comparing the blue and total samples), median $[\mathrm{Fe} / \mathrm{H}]$ values, and interquartile ranges (IQR) for each population. 


$$
(-5.5,-7)
$$

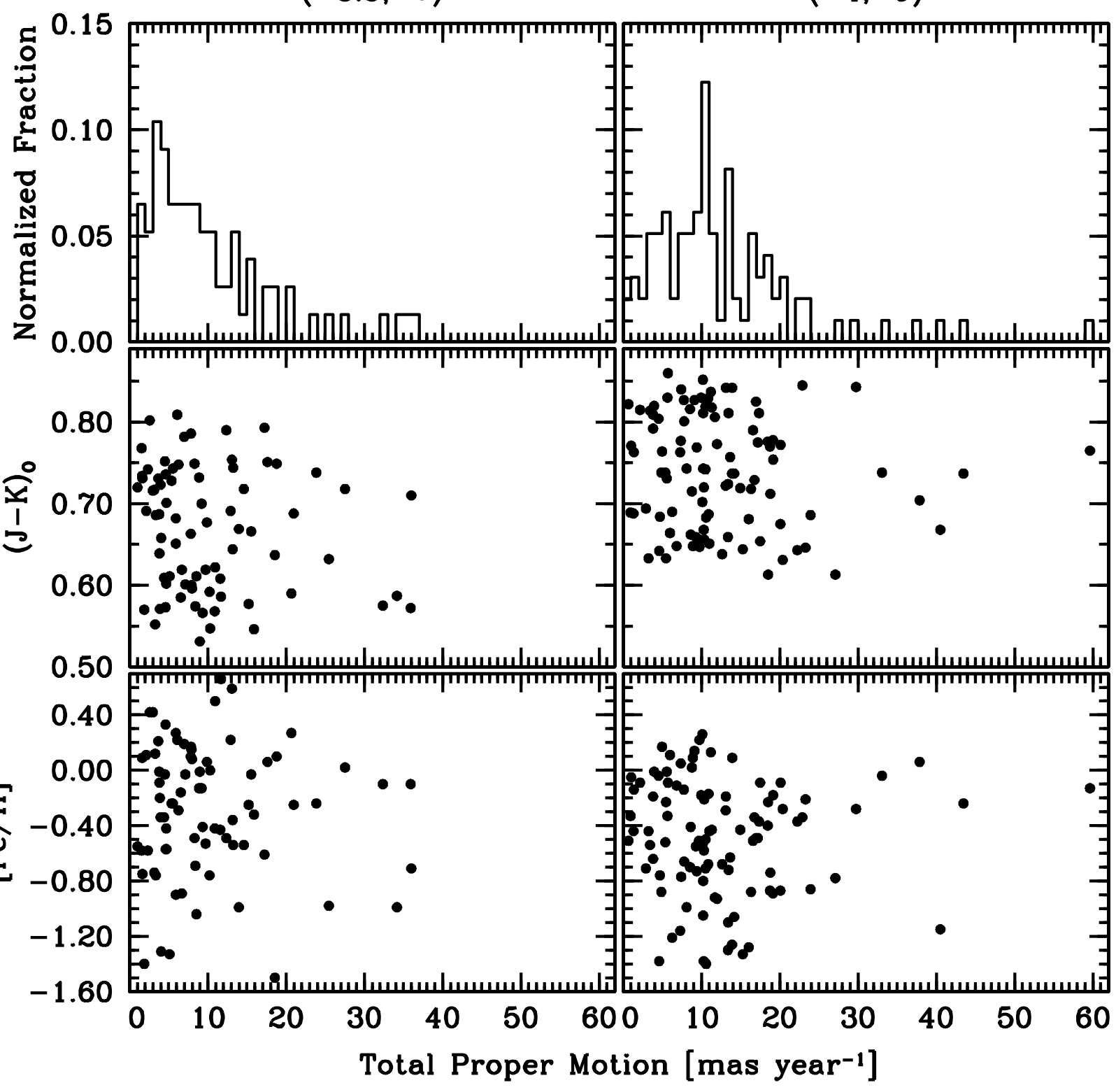

Fig. 11. - The left panels show (top) a histogram of the total proper motion for stars in the $(-5.5,-7)$ field, (middle) a plot of $(\mathrm{J}-\mathrm{K})_{\mathrm{o}}$ versus total proper motion, and (bottom) a plot of $[\mathrm{Fe} / \mathrm{H}]$ versus total proper motion. The right panels include the same information for the $(-4,-9)$ field. The proper motion data are from the SPM4 catalog. 


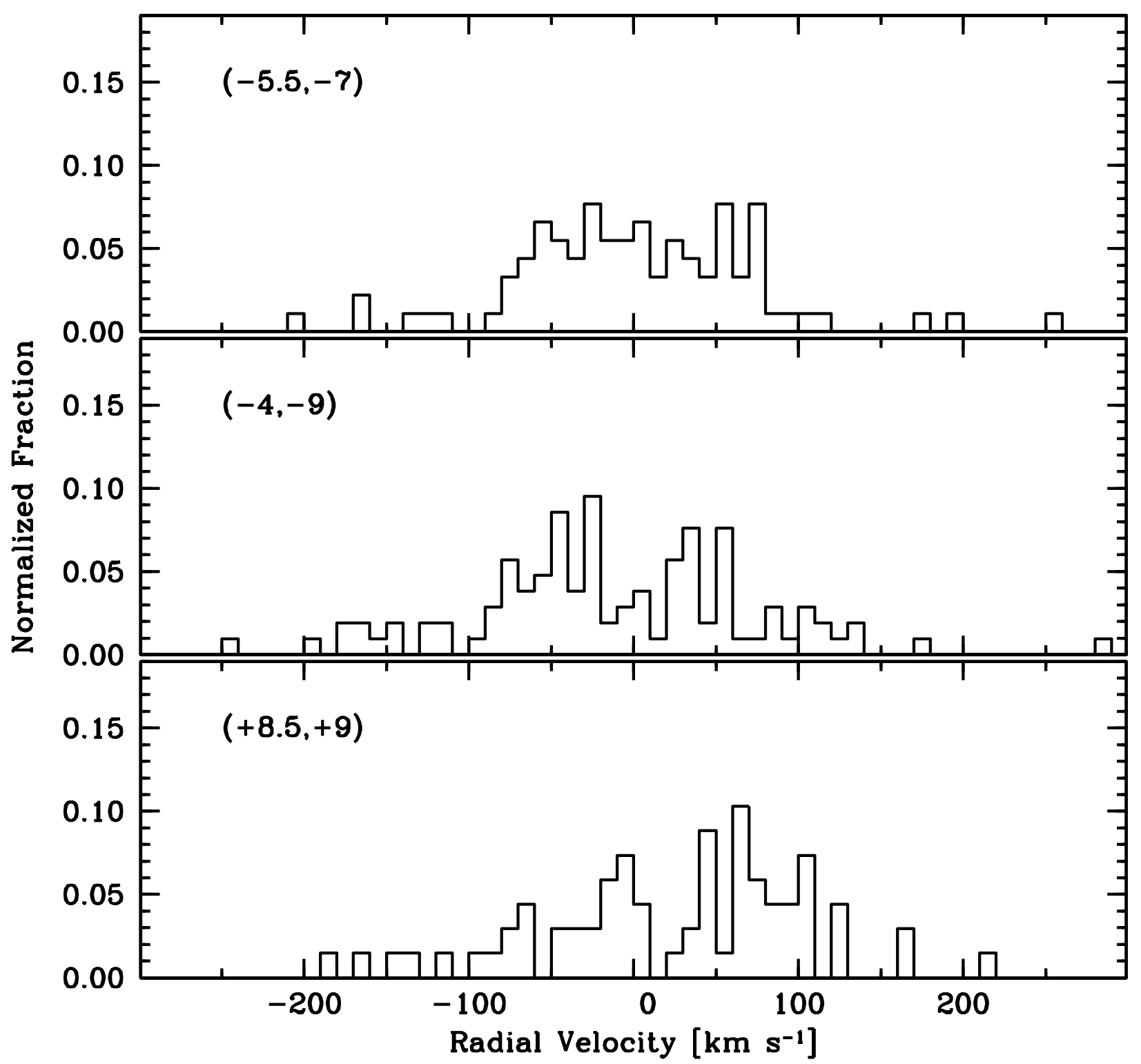

Fig. 12.- The three panels show the heliocentric radial velocity distributions for each field in $10 \mathrm{~km} \mathrm{~s}^{-1}$ bins. 


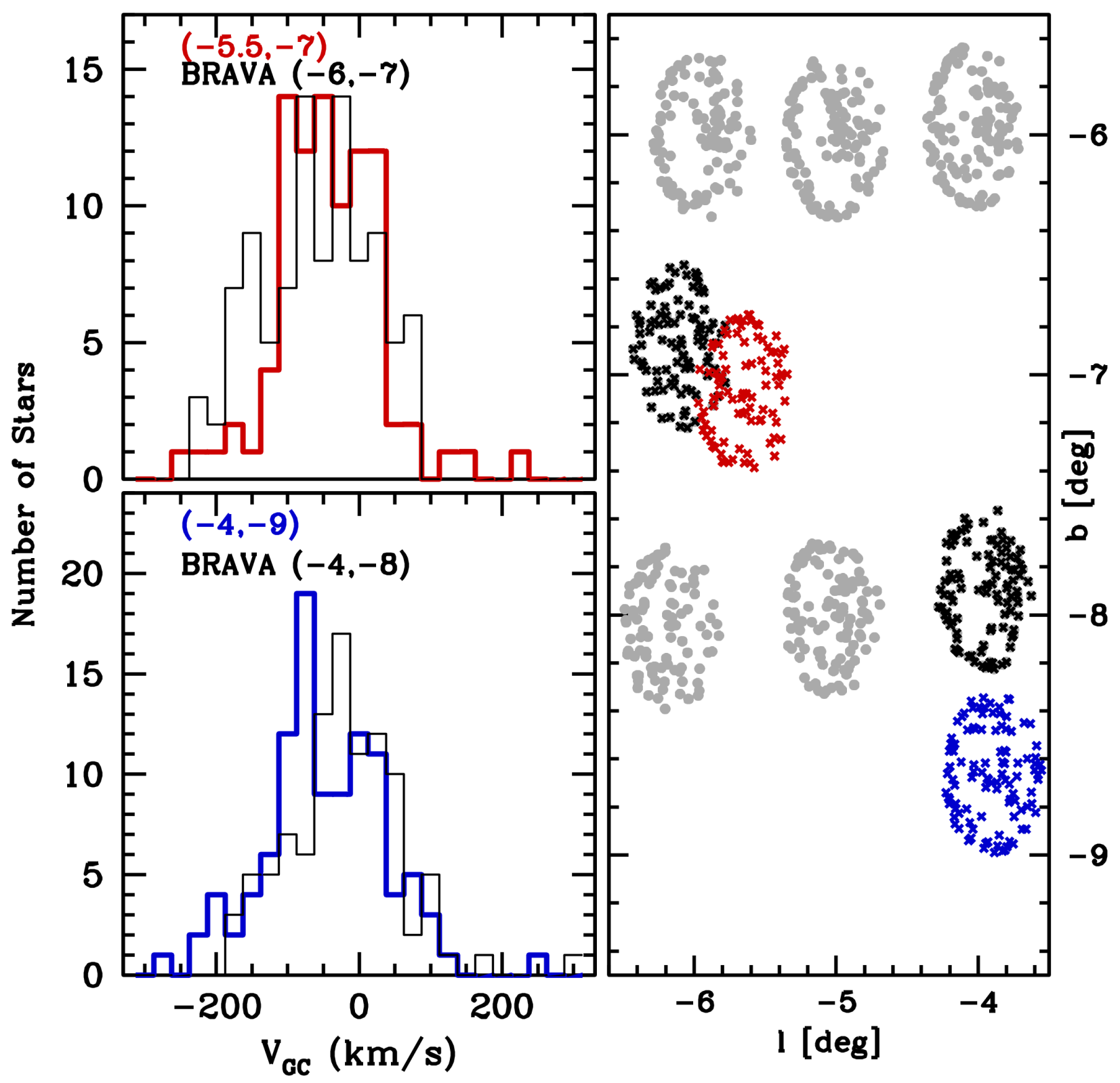

Fig. 13. - The top left and bottom left panels compare the (-5.5,-7; red lines and symbols) and $(-4,-9$; blue lines and symbols) to nearby and/or overlapping fields observed for the BRAVA project (Howard et al. 2008; Kunder et al. 2012). The data are binned in $25 \mathrm{~km}$ $\mathrm{s}^{-1}$ increments. The right panel shows the Galactic longitude and latitude for individual stars in the new fields observed here, the comparison BRAVA fields used in the histograms (black crosses), and other nearby BRAVA fields (grey filled circles). 


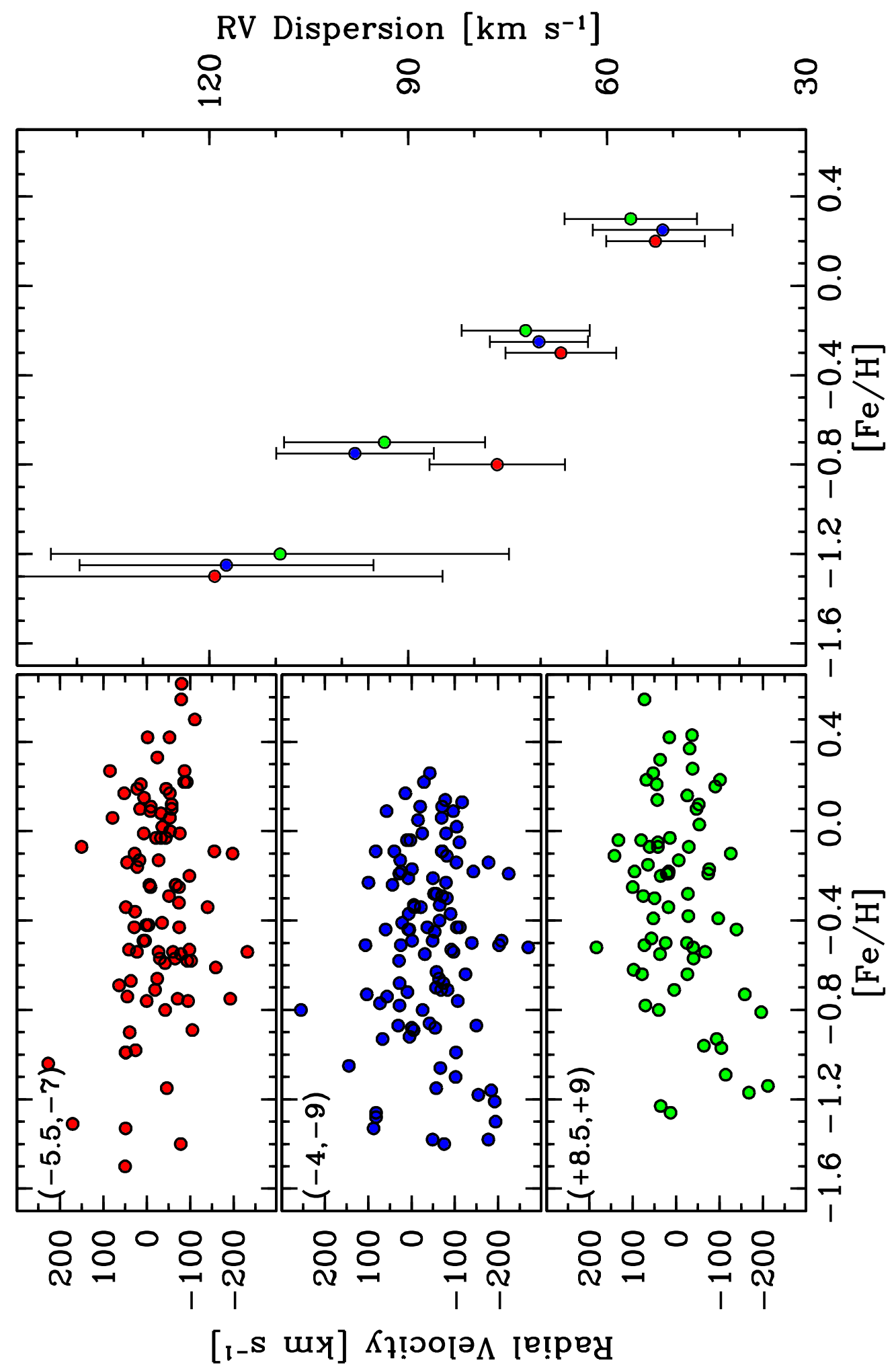

Fig. 14.- The left panels show the heliocentric radial velocity distribution as a function of $[\mathrm{Fe} / \mathrm{H}]$ for the $(-5.5,-7)$ (filled red circles), $(-4,-9)$ (filled blue circles), and $(+8.5,+9)$ (filled green circles) fields. In the right panel, the 38 locity dispersion is shown for all three fields with the data merged into $0.5 \mathrm{dex}[\mathrm{Fe} / \mathrm{H}]$ bins. The larger error bars on the most metal-poor bin are due to the relatively small sample size. 


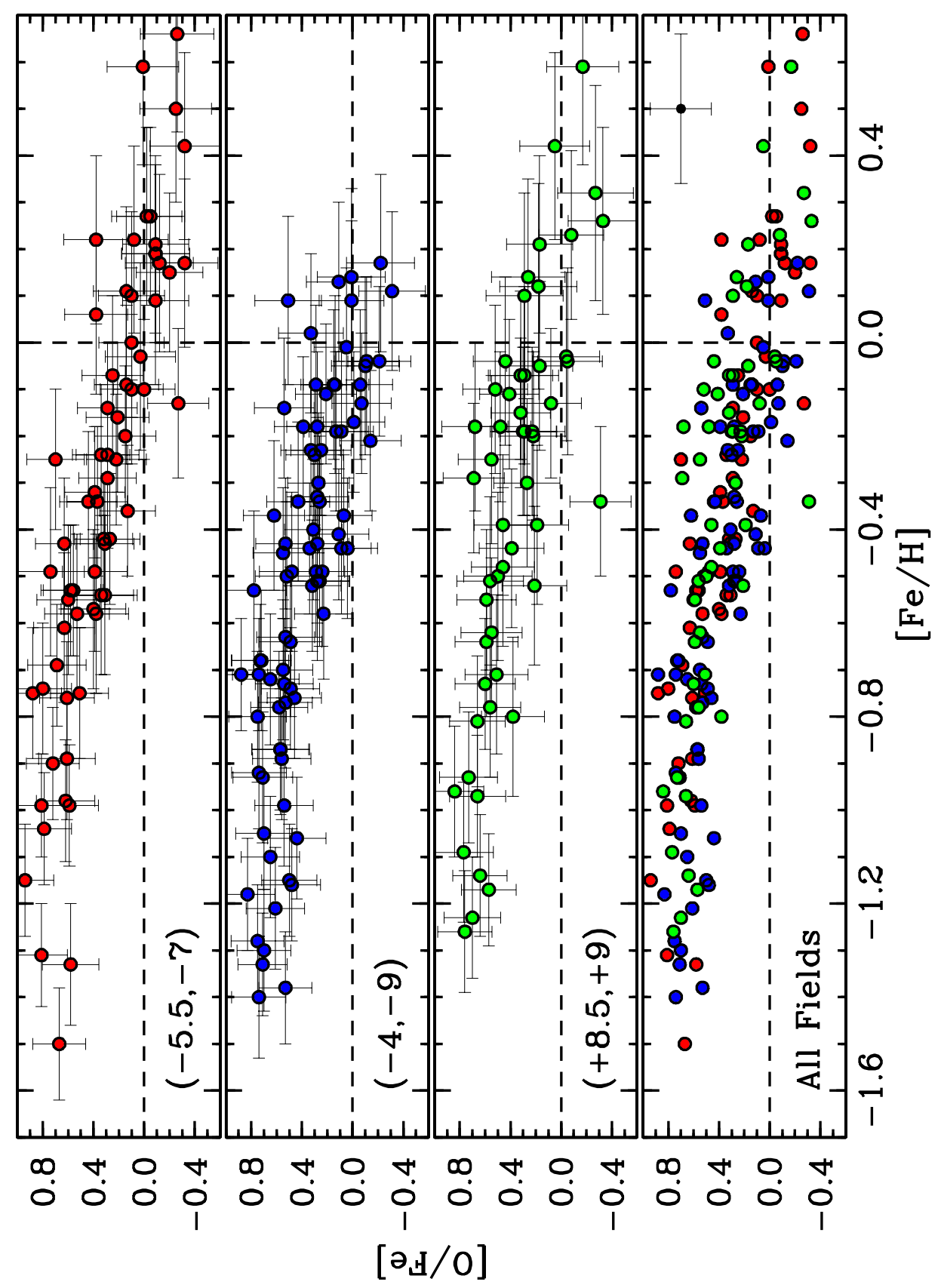

Fig. 15. $-[\mathrm{O} / \mathrm{Fe}]$ is plotted as a function of $[\mathrm{Fe} / \mathrm{H}]$ for all three fields. For display purposes, the individual error bars are suppressed in the bottom panel and a typical error bar is shown in the top right corner. 


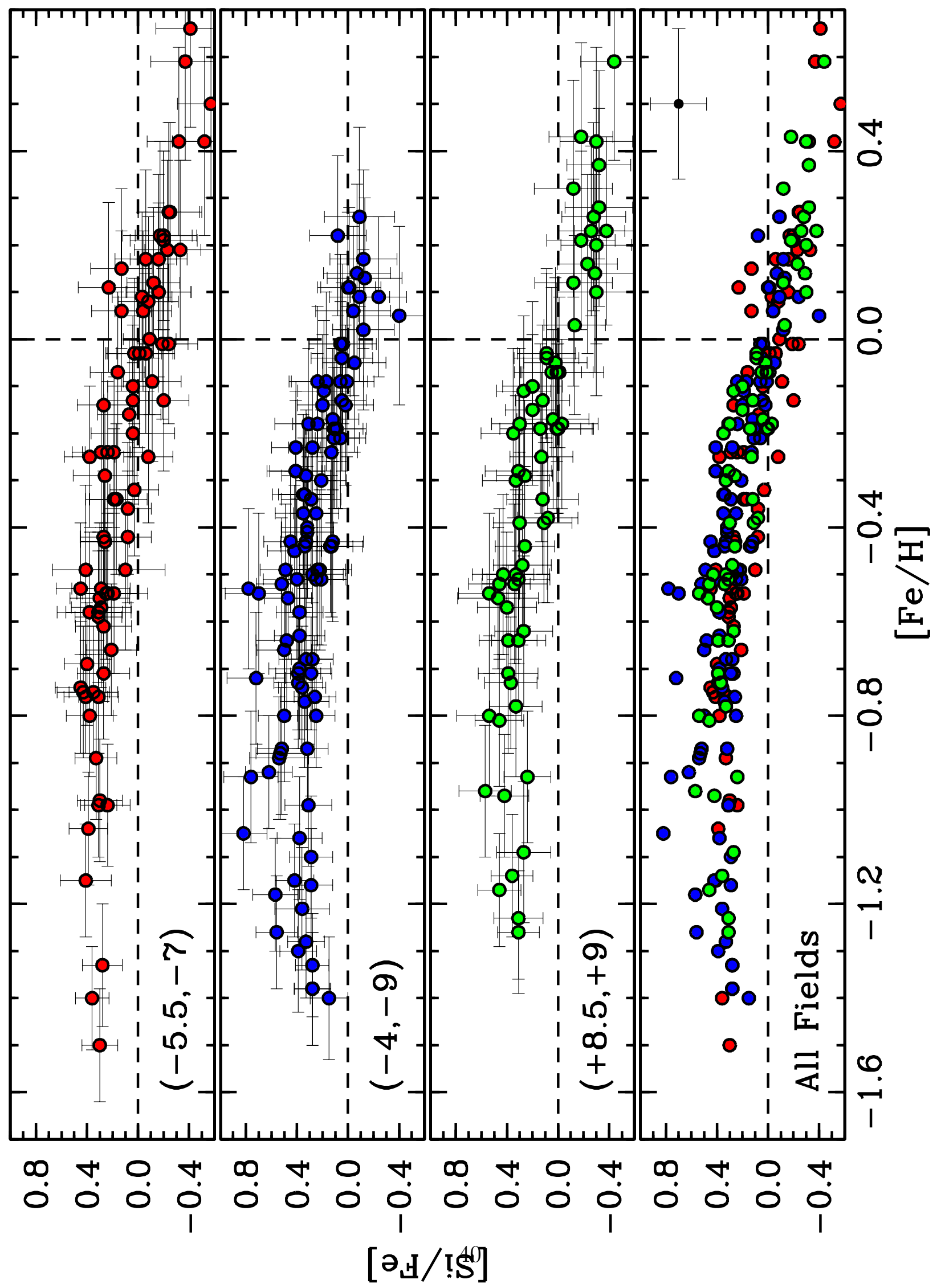

Fig. 16.- Similar to Figure 15 with $[\mathrm{Si} / \mathrm{Fe}]$ plotted as a function of $[\mathrm{Fe} / \mathrm{H}]$. 


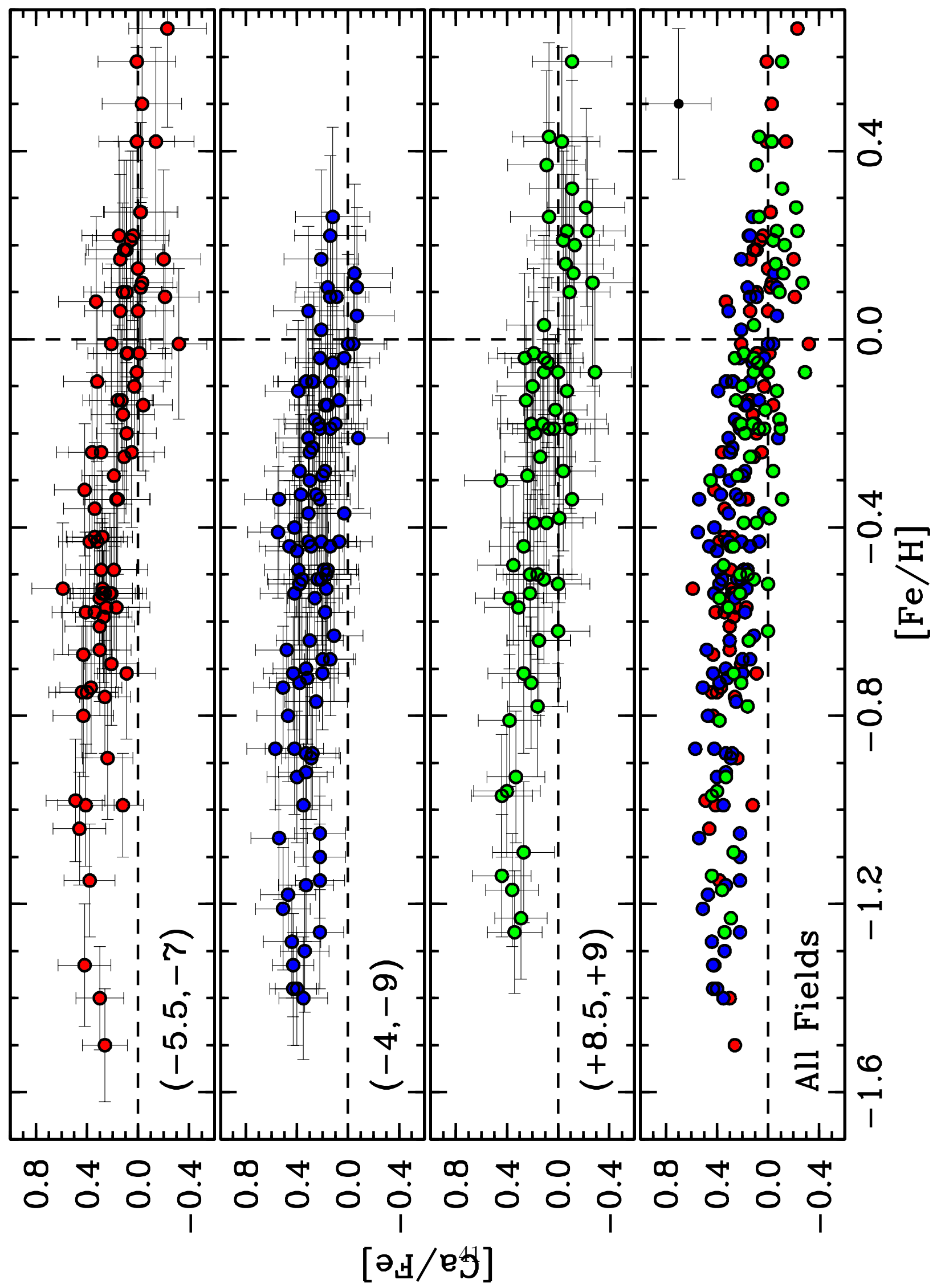

Fig. 17.- Similar to Figure 15 with $[\mathrm{Ca} / \mathrm{Fe}]$ plotted as a function of $[\mathrm{Fe} / \mathrm{H}]$. 


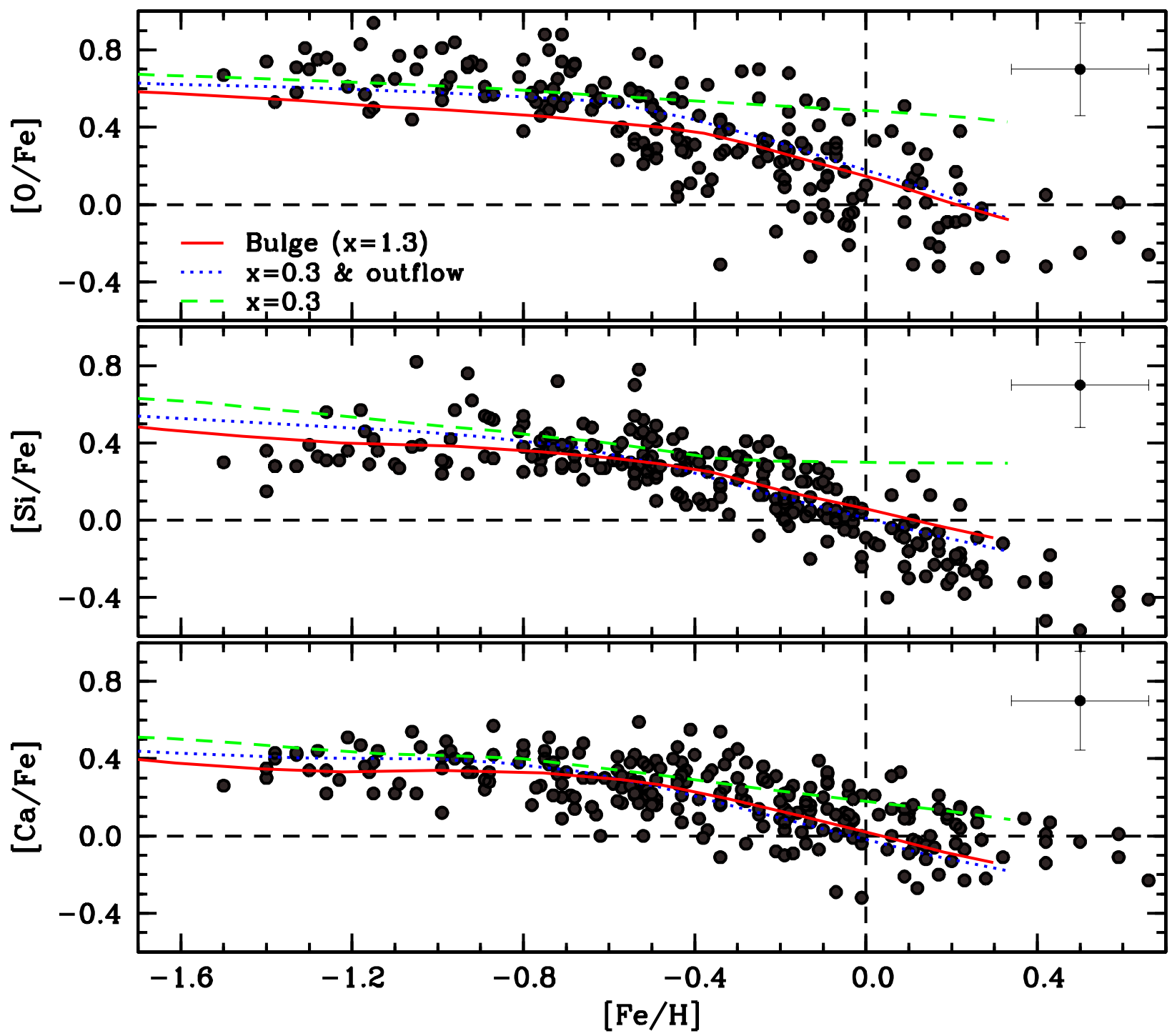

Fig. 18. - The $[\mathrm{O} / \mathrm{Fe}],[\mathrm{Si} / \mathrm{Fe}]$, and $[\mathrm{Ca} / \mathrm{Fe}]$ abundances for all three fields (combined) are plotted as a function of $[\mathrm{Fe} / \mathrm{H}]$. The solid red line shows the predicted change in each $\alpha$ element as a function of $[\mathrm{Fe} / \mathrm{H}]$, based on the bulge model of Kobayashi et al. (2011). The dashed green line is the model prediction assuming a flatter IMF $(x=0.3)$ and the dotted blue line is the model prediction assuming $\mathrm{x}=0.3$ with outflow. Note that the $[\mathrm{Si} / \mathrm{Fe}]$ model values have been systematically decreased by 0.2 dex (see text for details). 


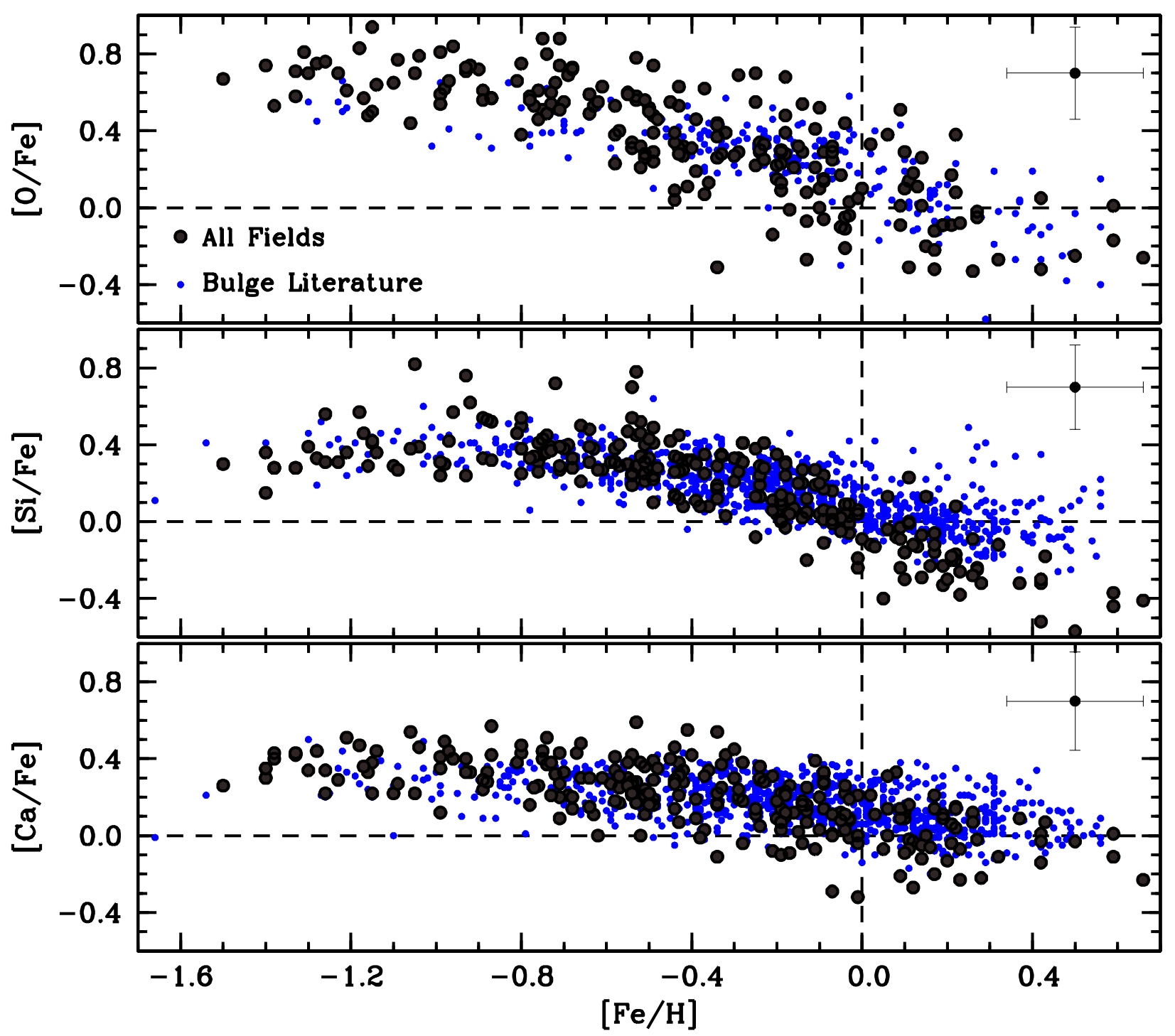

Fig. 19. - $[\mathrm{O} / \mathrm{Fe}],[\mathrm{Si} / \mathrm{Fe}]$, and $[\mathrm{Ca} / \mathrm{Fe}]$ ratios are plotted as a function of $[\mathrm{Fe} / \mathrm{H}]$ for all bulge stars measured here (filled grey circles) and compared with previous bulge measurements (filled blue circles). The individual error bars have been suppressed for display purposes and a typical error bar is shown in the top right corner of each panel. The literature data are from: McWilliam \& Rich (1994), Rich \& Origlia (2005), Fulbright et al. (2007), Lecureur et al. (2007), Rich et al. (2007b), Meléndez et al. (2008), Alves-Brito et al. (2010), Bensby et al. (2010a), Ryde et al. (2010), Bensby et al. (2011), Gonzalez et al. (2011), Johnson et al. (2011), and Rich et al. (2012). 


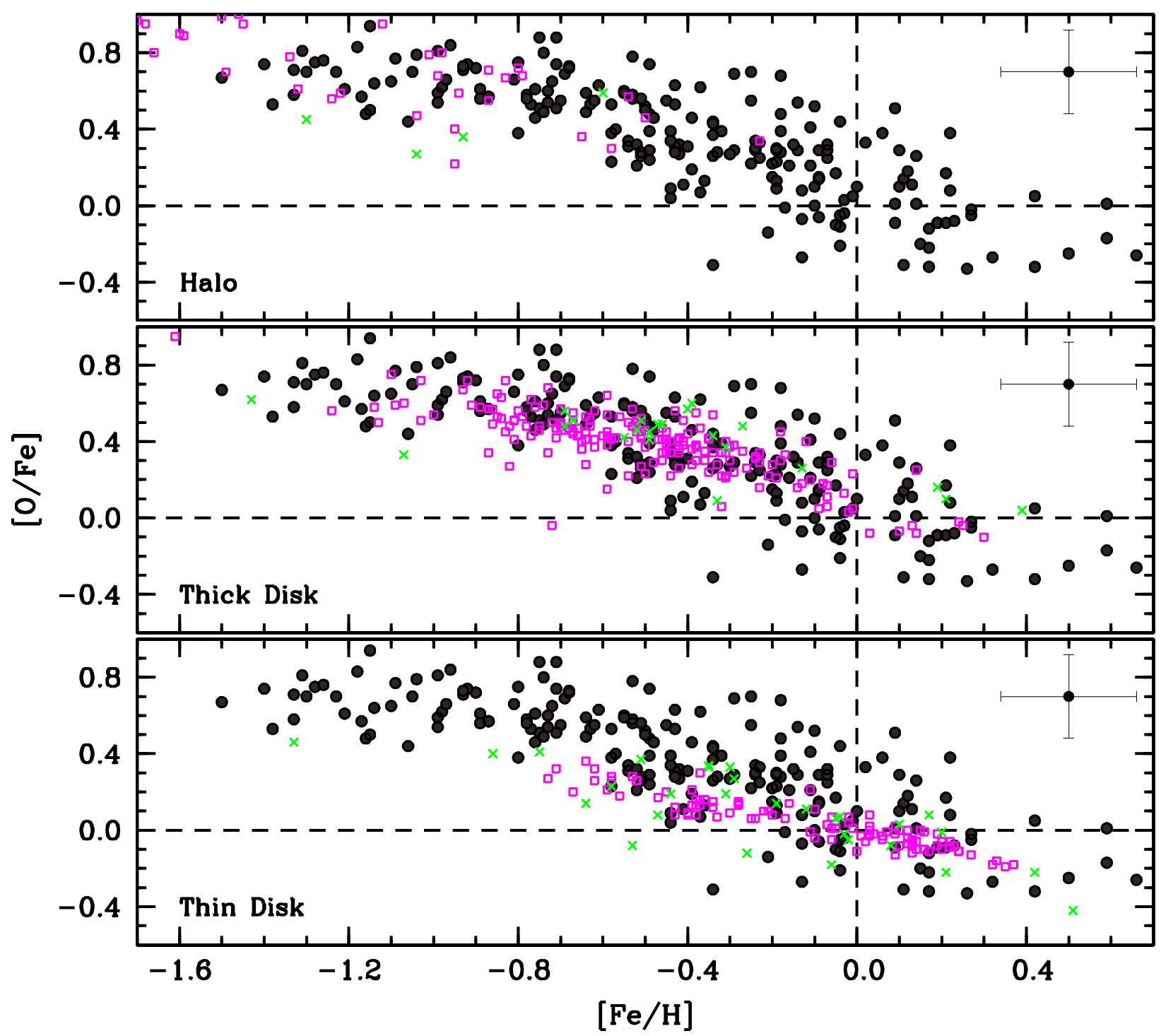

Fig. 20. $-[\mathrm{O} / \mathrm{Fe}]$ ratios are plotted as a function of $[\mathrm{Fe} / \mathrm{H}]$ for bulge stars in all three fields (filled grey circles), the Galactic halo (top panels), thick disk (middle panels), and thin disk (bottom panels). For the halo and disk populations, abundances derived from dwarfs and/or subgiants are designated by magenta open boxes and abundances derived from giants are designated by green crosses. The individual error bars for our bulge stars have been suppressed for display purposes and a typical error bar is shown in the top right corner of each panel. The halo and disk data are from: Tomkin et al. (1992), Edvardsson et al. (1993), Prochaska et al. (2000), Reddy et al. (2003), Bensby et al. (2005), Brewer \& Carney et al. (2006), Reddy et al. (2006), and Alves-Brito et al. (2010). For the Alves-Brito et al. (2010) giant data we show only the abundances derived using Kurucz atmospheres. 


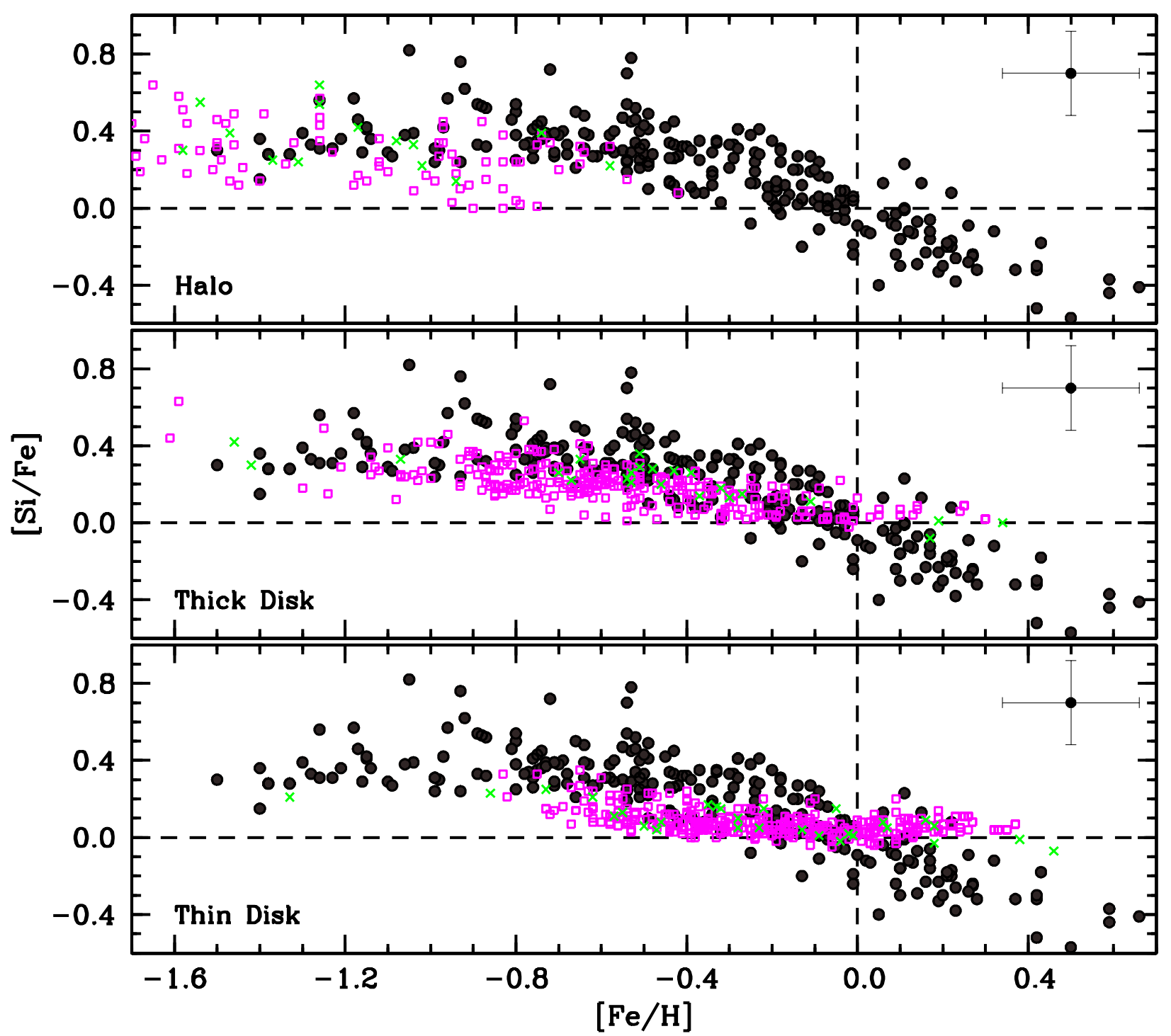

Fig. 21. - Similar to Figure 20 with $[\mathrm{Si} / \mathrm{Fe}]$ plotted as a function of $[\mathrm{Fe} / \mathrm{H}]$. The halo and disk data are from: Edvardsson et al. (1993), Nissen \& Schuster (1997), Fulbright (2000), Prochaska et al. (2000), Stephens \& Boesgaard (2002), Johnson (2002), Bensby et al. (2003), Reddy et al. (2003), Bensby et al. (2005), Brewer \& Carney et al. (2006), Reddy et al. (2006), and Alves-Brito et al. (2010). 


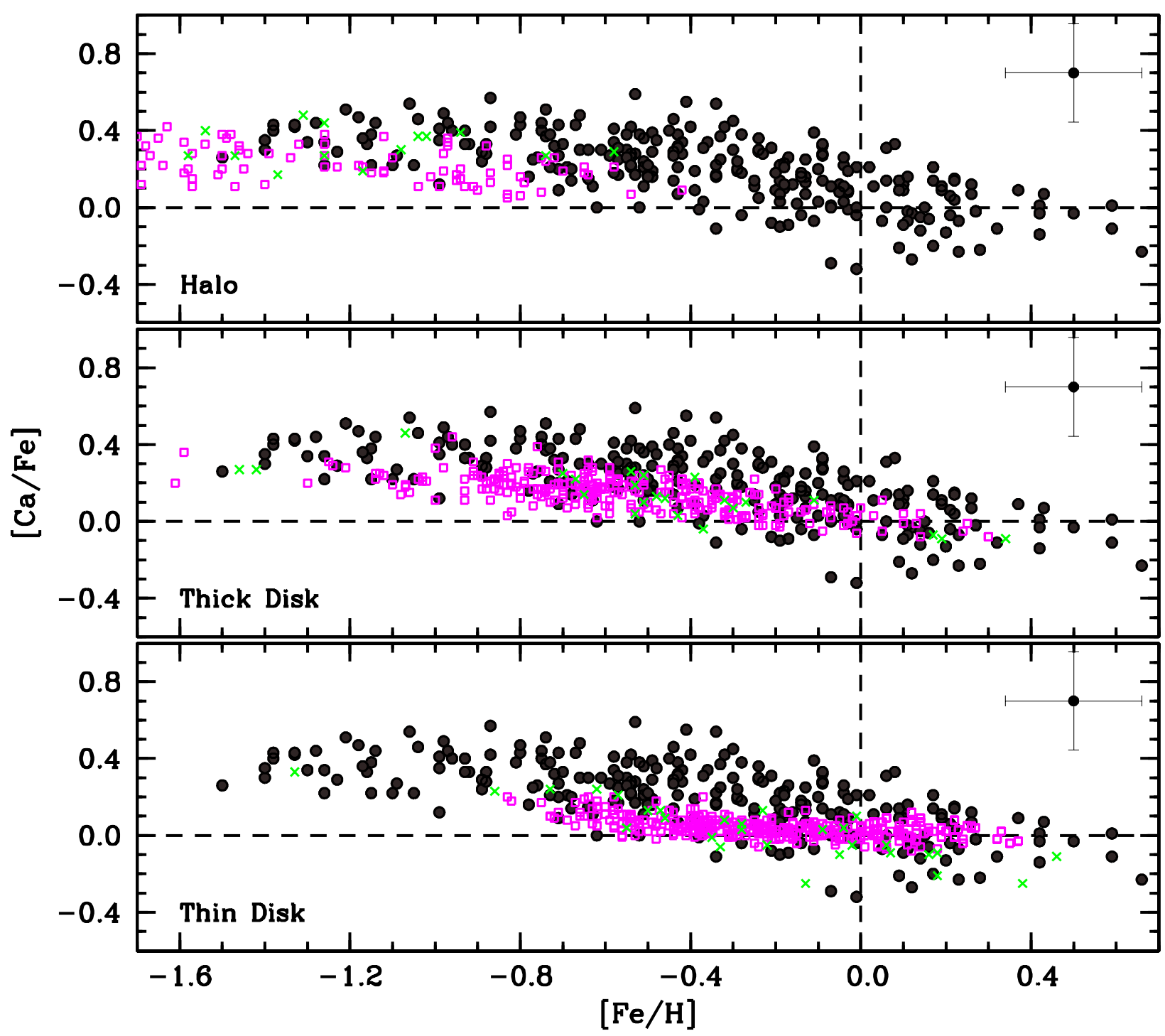

Fig. 22.- Similar to Figure 20 with $[\mathrm{Ca} / \mathrm{Fe}]$ plotted as a function of $[\mathrm{Fe} / \mathrm{H}]$. The literature data are from the same sources as in Figure 21. 
TABLE 1

Summary OF Hydra OBSERVATIONS

\begin{tabular}{ccccc}
\hline \hline UT Date & Telescope & Setup & Exposure & Field $(1, b)$ \\
\hline 2011 Aug 19 & WIYN & 6250 & $3 \times 3600 \mathrm{~s}$ & $(+8.5,+9)$ \\
2011 Aug 20 & WIYN & 6250 & $3 \times 3600 \mathrm{~s}$ & $(+8.5,+9)$ \\
2011 Sep 08 & Blanco & 6700 & $3 \times 3600 \mathrm{~s}$ & $(-5.5,-7)$ \\
2011 Sep 09 & Blanco & 6250 & $1 \times 2700 \mathrm{~s}$ & $(-5.5,-7)$ \\
2011 Sep 10 & Blanco & 6250 & $4 \times 3600 \mathrm{~s}$ & $(-5.5,-7)$ \\
2011 Sep 11 & Blanco & 6700 & $3 \times 3600 \mathrm{~s}$ & $(-4,-9)$ \\
2011 Sep 11 & Blanco & 6250 & $1 \times 3600 \mathrm{~s}$ & $(-4,-9)$ \\
2011 Sep 12 & Blanco & 6250 & $4 \times 3600 \mathrm{~s}$ & $(-4,-9)$ \\
\hline
\end{tabular}


TABle 2

Star Identifiers, Photometry, Model Atmosphere Parameters, and Radial Velocities

\begin{tabular}{|c|c|c|c|c|c|c|c|c|c|}
\hline $\begin{array}{l}\text { Star ID } \\
2 \mathrm{MASS}\end{array}$ & $\mathrm{E}(\mathrm{B}-\mathrm{V})$ & $\mathrm{J}$ & $\mathrm{H}$ & $\mathrm{K}_{\mathrm{S}}$ & $\begin{array}{l}\mathrm{T}_{\text {eff }} \\
(\mathrm{K})\end{array}$ & $\begin{array}{c}\log (g) \\
(\operatorname{cgs})\end{array}$ & {$[\mathrm{Fe} / \mathrm{H}]$} & $\begin{array}{c}\mathrm{V}_{\mathrm{t}} \\
\left(\mathrm{km} \mathrm{s}^{-1}\right)\end{array}$ & $\begin{array}{c}\text { RV Helio. } \\
\left(\mathrm{km} \mathrm{s}^{-1}\right)\end{array}$ \\
\hline \multicolumn{10}{|c|}{$(1, b)=(-5.5,-7)$} \\
\hline $18011989-3723462$ & 0.212 & 10.996 & 10.313 & 10.148 & 4405 & 1.20 & -1.50 & 1.55 & +50.3 \\
\hline $18003452-3729217$ & 0.232 & 11.809 & 11.142 & 11.022 & 4605 & 1.60 & -1.40 & 2.15 & -78.2 \\
\hline $18011692-3722508$ & 0.222 & 11.436 & 10.753 & 10.608 & 4475 & 1.45 & -1.33 & 1.75 & +49.0 \\
\hline $18005584-3739103$ & 0.212 & 11.525 & 10.805 & 10.659 & 4360 & 1.30 & -1.31 & 1.80 & +171.3 \\
\hline $18015010-3735279$ & 0.196 & 11.568 & 10.880 & 10.736 & 4425 & 1.50 & -1.15 & 1.80 & -45.9 \\
\hline $18005916-3727585$ & 0.220 & 11.610 & 10.935 & 10.796 & 4510 & 1.70 & -1.04 & 1.55 & +227.8 \\
\hline $18005027-3707505$ & 0.268 & 11.908 & 11.261 & 11.110 & 4630 & 1.90 & -0.99 & 1.85 & +48.4 \\
\hline $18005234-3715478$ & 0.250 & 11.168 & 10.407 & 10.294 & 4395 & 1.55 & -0.99 & 1.40 & +49.2 \\
\hline $18020106-3737561$ & 0.203 & 11.266 & 10.568 & 10.416 & 4390 & 1.55 & -0.98 & 1.65 & +26.0 \\
\hline $17595541-3713447$ & 0.324 & 11.425 & 10.688 & 10.558 & 4520 & 1.75 & -0.90 & 1.45 & +39.7 \\
\hline $17595664-3725304$ & 0.268 & 12.034 & 11.318 & 11.220 & 4585 & 1.85 & -0.89 & 1.20 & -104.7 \\
\hline $18021345-3730371$ & 0.191 & 11.669 & 10.910 & 10.748 & 4200 & 1.35 & -0.80 & 1.90 & -42.4 \\
\hline $17594241-3718247$ & 0.311 & 11.436 & 10.669 & 10.529 & 4395 & 1.60 & -0.76 & 2.15 & -94.9 \\
\hline $18013342-3726339$ & 0.202 & 11.784 & 11.095 & 10.979 & 4505 & 1.75 & -0.76 & 1.90 & +0.5 \\
\hline $18003204-3726008$ & 0.243 & 10.558 & 9.755 & 9.587 & 4150 & 1.25 & -0.75 & 1.55 & -70.9 \\
\hline $18013580-3740055$ & 0.211 & 11.383 & 10.589 & 10.450 & 4195 & 1.35 & -0.75 & 1.80 & -192.4 \\
\hline $18013799-3725131$ & 0.201 & 11.349 & 10.591 & 10.412 & 4175 & 1.35 & -0.74 & 1.60 & +45.0 \\
\hline $18022149-3713494$ & 0.223 & 11.033 & 10.276 & 10.106 & 4225 & 1.40 & -0.71 & 1.75 & -18.9 \\
\hline $18011408-3711299$ & 0.237 & 12.001 & 11.310 & 11.203 & 4580 & 1.95 & -0.69 & 1.40 & +63.8 \\
\hline $18021275-3737543$ & 0.206 & 11.938 & 11.261 & 11.137 & 4525 & 1.85 & -0.67 & 1.45 & +36.9 \\
\hline $17595027-3727293$ & 0.265 & 11.679 & 10.864 & 10.744 & 4260 & 1.60 & -0.66 & 1.65 & -23.9 \\
\hline $18002115-3715519$ & 0.276 & 11.041 & 10.212 & 10.035 & 4115 & 1.30 & -0.61 & 1.55 & -158.9 \\
\hline $17595366-3728186$ & 0.266 & 11.857 & 11.216 & 11.019 & 4515 & 1.85 & -0.59 & 1.85 & -41.9 \\
\hline
\end{tabular}


TABLE 2-Continued

\begin{tabular}{|c|c|c|c|c|c|c|c|c|c|}
\hline $\begin{array}{l}\text { Star ID } \\
2 \mathrm{MASS}\end{array}$ & $\mathrm{E}(\mathrm{B}-\mathrm{V})$ & $\mathrm{J}$ & $\mathrm{H}$ & $\mathrm{K}_{\mathrm{S}}$ & $\begin{array}{l}\mathrm{T}_{\text {eff }} \\
(\mathrm{K})\end{array}$ & $\begin{array}{c}\log (g) \\
(\operatorname{cgs})\end{array}$ & {$[\mathrm{Fe} / \mathrm{H}]$} & $\begin{array}{c}\mathrm{V}_{\mathrm{t}} \\
\left(\mathrm{km} \mathrm{s}^{-1}\right)\end{array}$ & $\begin{array}{r}\text { RV Helio. } \\
\left(\mathrm{km} \mathrm{s}^{-1}\right)\end{array}$ \\
\hline $18001354-3709122$ & 0.292 & 11.414 & 10.624 & 10.428 & 4175 & 1.50 & -0.58 & 1.65 & -101.9 \\
\hline $18013960-3723398$ & 0.211 & 11.616 & 10.847 & 10.669 & 4165 & 1.50 & -0.58 & 1.10 & -93.3 \\
\hline $18021862-3734204$ & 0.196 & 11.856 & 11.058 & 10.938 & 4210 & 1.55 & -0.57 & 1.55 & -30.0 \\
\hline $18000535-3735315$ & 0.226 & 10.115 & 9.348 & 9.163 & 4175 & 1.40 & -0.57 & 1.55 & -64.9 \\
\hline $18013848-3721442$ & 0.216 & 11.798 & 11.086 & 10.878 & 4230 & 1.55 & -0.55 & 1.70 & -78.5 \\
\hline $17594792-3721422$ & 0.316 & 10.871 & 10.128 & 9.954 & 4375 & 1.70 & -0.54 & 1.40 & -26.7 \\
\hline $18011941-3721250$ & 0.222 & 11.476 & 10.736 & 10.518 & 4155 & 1.45 & -0.54 & 2.00 & -231.8 \\
\hline $18004824-3718074$ & 0.251 & 11.531 & 10.714 & 10.539 & 4115 & 1.40 & -0.54 & 1.50 & -60.3 \\
\hline $18014343-3708232$ & 0.233 & 11.140 & 10.348 & 10.190 & 4185 & 1.50 & -0.54 & 1.35 & +22.8 \\
\hline $18005852-3711524$ & 0.252 & 11.873 & 11.260 & 11.065 & 4575 & 1.95 & -0.53 & 1.35 & -97.7 \\
\hline $18020816-3714015$ & 0.223 & 12.097 & 11.433 & 11.280 & 4505 & 1.85 & -0.53 & 1.60 & +41.4 \\
\hline $18010932-3702079$ & 0.262 & 11.050 & 10.291 & 10.065 & 4140 & 1.45 & -0.49 & 1.80 & +7.8 \\
\hline $18010065-3740456$ & 0.219 & 11.362 & 10.570 & 10.397 & 4135 & 1.45 & -0.49 & 1.55 & +4.2 \\
\hline $17595640-3710383$ & 0.326 & 10.761 & 9.998 & 9.836 & 4370 & 1.70 & -0.43 & 1.30 & +29.0 \\
\hline $18012462-3738382$ & 0.205 & 11.950 & 11.271 & 11.136 & 4485 & 1.85 & -0.43 & 1.90 & -74.6 \\
\hline $18002880-3710171$ & 0.274 & 11.899 & 11.253 & 11.098 & 4630 & 2.00 & -0.42 & 1.80 & +1.7 \\
\hline $18024484-3714159$ & 0.233 & 12.197 & 11.538 & 11.412 & 4615 & 2.00 & -0.42 & 1.35 & -3.8 \\
\hline $18013206-3708133$ & 0.235 & 12.106 & 11.451 & 11.322 & 4620 & 2.00 & -0.41 & 1.40 & -35.0 \\
\hline $18004074-3740058$ & 0.219 & 11.129 & 10.399 & 10.269 & 4385 & 1.75 & -0.36 & 1.80 & +27.5 \\
\hline $18000013-3715053$ & 0.297 & 11.412 & 10.663 & 10.486 & 4325 & 1.75 & -0.34 & 1.60 & +48.6 \\
\hline $18015431-3717070$ & 0.216 & 11.792 & 11.143 & 10.965 & 4470 & 1.90 & -0.34 & 1.30 & -139.9 \\
\hline $18021006-3738193$ & 0.206 & 11.920 & 11.261 & 11.161 & 4645 & 2.05 & -0.32 & 1.65 & -73.8 \\
\hline $17595442-3721118$ & 0.291 & 11.300 & 10.532 & 10.352 & 4265 & 1.60 & -0.29 & 1.60 & -51.2 \\
\hline $18021721-3736096$ & 0.202 & 11.735 & 11.078 & 10.962 & 4600 & 2.00 & -0.25 & 1.90 & -8.4 \\
\hline
\end{tabular}


TABLE 2-Continued

\begin{tabular}{cccccccccc}
\hline \hline Star ID & $\mathrm{E}(\mathrm{B}-\mathrm{V})$ & $\mathrm{J}$ & $\mathrm{H}$ & $\mathrm{K}_{\mathrm{S}}$ & $\begin{array}{c}\mathrm{T}_{\text {eff }} \\
(\mathrm{K})\end{array}$ & $\begin{array}{c}\log (\mathrm{g}) \\
(\mathrm{cgs})\end{array}$ & {$[\mathrm{Fe} / \mathrm{H}]$} & $\begin{array}{c}\mathrm{V}_{\mathrm{t}} \\
\left(\mathrm{km} \mathrm{s}^{-1}\right)\end{array}$ & $\begin{array}{c}\text { RV Helio. } \\
\left(\mathrm{km} \mathrm{s}^{-1}\right)\end{array}$ \\
\hline $18005096-3733499$ & 0.215 & 11.899 & 11.122 & 10.995 & 4270 & 1.70 & -0.25 & 1.25 & -74.1 \\
$18005923-3707111$ & 0.262 & 11.534 & 10.744 & 10.575 & 4200 & 1.65 & -0.24 & 1.30 & -66.3 \\
$18011851-3706092$ & 0.248 & 11.093 & 10.324 & 10.146 & 4210 & 1.65 & -0.24 & 1.65 & -67.1 \\
$18013395-3705551$ & 0.236 & 10.910 & 10.147 & 9.954 & 4175 & 1.60 & -0.24 & 1.35 & -5.7 \\
$18022648-3728188$ & 0.204 & 11.921 & 11.249 & 11.132 & 4555 & 1.95 & -0.20 & 1.95 & -98.1 \\
$18024919-3727234$ & 0.215 & 11.553 & 10.958 & 10.772 & 4595 & 2.00 & -0.16 & 1.85 & +22.4 \\
$18023660-3716289$ & 0.231 & 12.155 & 11.471 & 11.360 & 4580 & 1.95 & -0.14 & 1.90 & +45.6 \\
$17595509-3711099$ & 0.326 & 10.786 & 10.066 & 9.871 & 4395 & 1.80 & -0.13 & 1.80 & -27.1 \\
$18005395-3731231$ & 0.215 & 9.731 & 9.003 & 8.783 & 4165 & 1.60 & -0.13 & 1.90 & +17.3 \\
$18010322-3705447$ & 0.264 & 12.067 & 11.444 & 11.276 & 4645 & 2.05 & -0.10 & 1.80 & +28.4 \\
$18014769-3739220$ & 0.210 & 12.128 & 11.416 & 11.356 & 4615 & 2.00 & -0.10 & 1.20 & -197.7 \\
$17594807-3715283$ & 0.335 & 11.520 & 10.793 & 10.662 & 4565 & 2.00 & -0.09 & 1.35 & -155.7 \\
$18013618-3706311$ & 0.234 & 11.292 & 10.565 & 10.373 & 4260 & 1.70 & -0.07 & 1.50 & +150.7 \\
$18004314-3730156$ & 0.222 & 11.892 & 11.223 & 11.074 & 4500 & 1.95 & -0.03 & 1.45 & -21.1 \\
$18014303-3740054$ & 0.212 & 10.738 & 10.030 & 9.854 & 4315 & 1.75 & -0.03 & 1.75 & -32.6 \\
$18003751-3728200$ & 0.232 & 10.465 & 9.657 & 9.490 & 4130 & 1.55 & -0.03 & 1.30 & -43.5 \\
$18011170-3727118$ & 0.213 & 12.157 & 11.555 & 11.409 & 4690 & 2.05 & -0.01 & 1.85 & +7.1 \\
$18023846-3718345$ & 0.228 & 11.903 & 11.175 & 11.008 & 4310 & 1.80 & -0.01 & 1.90 & -76.0 \\
$18011671-3722213$ & 0.222 & 12.034 & 11.424 & 11.273 & 4665 & 2.05 & +0.00 & 1.60 & -53.6 \\
$18005985-3734507$ & 0.208 & 11.526 & 10.762 & 10.590 & 4185 & 1.75 & +0.02 & 1.40 & -35.6 \\
$18000430-3711051$ & 0.311 & 10.596 & 9.803 & 9.629 & 4245 & 1.70 & +0.06 & 1.60 & +79.2 \\
$18003552-3729476$ & 0.226 & 11.270 & 10.506 & 10.376 & 4310 & 1.80 & +0.06 & 1.75 & -53.2 \\
$18001817-3732243$ & 0.238 & 11.710 & 11.056 & 10.896 & 4535 & 1.95 & +0.08 & 1.35 & -32.6 \\
$18013039-3713086$ & 0.234 & 11.301 & 10.574 & 10.374 & 4240 & 1.80 & +0.09 & 2.00 & -8.3
\end{tabular}


TABLE 2-Continued

\begin{tabular}{|c|c|c|c|c|c|c|c|c|c|}
\hline $\begin{array}{l}\text { Star ID } \\
2 \mathrm{MASS}\end{array}$ & $\mathrm{E}(\mathrm{B}-\mathrm{V})$ & $\mathrm{J}$ & $\mathrm{H}$ & $\mathrm{K}_{\mathrm{S}}$ & $\begin{array}{l}\mathrm{T}_{\text {eff }} \\
(\mathrm{K})\end{array}$ & $\begin{array}{c}\log (g) \\
(\operatorname{cgs})\end{array}$ & {$[\mathrm{Fe} / \mathrm{H}]$} & $\begin{array}{c}\mathrm{V}_{\mathrm{t}} \\
\left(\mathrm{km} \mathrm{s}^{-1}\right)\end{array}$ & $\begin{array}{c}\text { RV Helio. } \\
\left(\mathrm{km} \mathrm{s}^{-1}\right)\end{array}$ \\
\hline $18012564-3741052$ & 0.215 & 11.135 & 10.427 & 10.256 & 4330 & 1.85 & +0.10 & 1.90 & -57.1 \\
\hline $18025713-3718318$ & 0.233 & 9.999 & 9.250 & 9.049 & 4185 & 1.60 & +0.10 & 1.70 & +15.7 \\
\hline $18011599-3741385$ & 0.219 & 11.408 & 10.679 & 10.514 & 4300 & 1.80 & +0.11 & 1.50 & -9.3 \\
\hline $18012944-3719488$ & 0.225 & 12.181 & 11.576 & 11.419 & 4670 & 2.05 & +0.12 & 1.95 & -57.2 \\
\hline $18020361-3719086$ & 0.210 & 12.007 & 11.363 & 11.190 & 4485 & 1.95 & +0.15 & 1.90 & +6.5 \\
\hline $18024024-3728187$ & 0.208 & 11.803 & 11.088 & 10.870 & 4195 & 1.75 & +0.17 & 1.80 & +52.4 \\
\hline $18000412-3717266$ & 0.288 & 11.110 & 10.320 & 10.111 & 4145 & 1.70 & +0.17 & 1.70 & -52.9 \\
\hline $18011177-3703344$ & 0.270 & 10.829 & 10.123 & 9.895 & 4270 & 1.80 & +0.19 & 1.60 & -44.5 \\
\hline $18001410-3725181$ & 0.256 & 10.701 & 9.920 & 9.705 & 4110 & 1.65 & +0.19 & 1.40 & +22.5 \\
\hline $18020300-3719418$ & 0.205 & 10.172 & 9.420 & 9.222 & 4150 & 1.70 & +0.21 & 1.20 & +14.0 \\
\hline $18004683-3703252$ & 0.286 & 11.522 & 10.766 & 10.504 & 4100 & 1.65 & +0.22 & 1.50 & -92.0 \\
\hline $18015404-3722564$ & 0.201 & 10.959 & 10.260 & 10.059 & 4260 & 1.75 & +0.22 & 1.55 & -86.0 \\
\hline $18013974-3704068$ & 0.233 & 10.406 & 9.687 & 9.506 & 4305 & 1.80 & +0.27 & 1.60 & +85.0 \\
\hline $18020704-3708064$ & 0.226 & 11.494 & 10.860 & 10.688 & 4540 & 1.95 & +0.27 & 1.60 & -86.8 \\
\hline $18023393-3730273$ & 0.204 & 11.730 & 11.125 & 10.948 & 4575 & 2.00 & +0.33 & 1.90 & -24.3 \\
\hline $18001720-3708374$ & 0.292 & 10.695 & 9.928 & 9.676 & 4105 & 1.70 & +0.42 & 1.50 & -52.4 \\
\hline $18003663-3708547$ & 0.268 & 11.057 & 10.348 & 10.134 & 4295 & 1.80 & +0.42 & 1.40 & -1.5 \\
\hline $18002569-3729578$ & 0.240 & 11.855 & 11.251 & 11.030 & 4510 & 2.00 & +0.50 & 1.50 & -110.6 \\
\hline $17594881-3716203$ & 0.322 & 10.708 & 9.952 & 9.736 & 4250 & 1.85 & +0.59 & 1.45 & -79.3 \\
\hline $18011201-3740090$ & 0.209 & 11.423 & 10.789 & 10.621 & 4525 & 2.00 & +0.66 & 1.65 & -80.4 \\
\hline \multicolumn{10}{|c|}{$(\mathrm{l}, \mathrm{b})=(-4,-9)$} \\
\hline $18122552-3653049$ & 0.139 & 11.527 & 10.906 & 10.775 & 4560 & 1.55 & -1.40 & 1.40 & -75.2 \\
\hline $18120653-3654264$ & 0.143 & 12.095 & 11.497 & 11.376 & 4660 & 1.65 & -1.38 & 1.00 & -48.6 \\
\hline $18110179-3630394$ & 0.138 & 12.165 & 11.540 & 11.418 & 4570 & 1.65 & -1.38 & 1.55 & -177.3 \\
\hline
\end{tabular}


TABLE 2-Continued

\begin{tabular}{cccccccccc}
\hline \hline Star ID & $\mathrm{E}(\mathrm{B}-\mathrm{V})$ & $\mathrm{J}$ & $\mathrm{H}$ & $\mathrm{K}_{\mathrm{S}}$ & $\begin{array}{c}\mathrm{T}_{\text {eff }} \\
(\mathrm{K})\end{array}$ & $\begin{array}{c}\log (\mathrm{g}) \\
(\mathrm{cgs})\end{array}$ & {$[\mathrm{Fe} / \mathrm{H}]$} & $\begin{array}{c}\mathrm{V}_{\mathrm{t}} \\
\left(\mathrm{km} \mathrm{s}^{-1}\right)\end{array}$ & $\begin{array}{c}\text { RV Helio. } \\
\left(\mathrm{km} \mathrm{s}^{-1}\right)\end{array}$ \\
\hline $18124892-3626403$ & 0.140 & 11.787 & 11.170 & 11.066 & 4650 & 1.75 & -1.33 & 1.50 & +87.4 \\
$18135164-3643463$ & 0.119 & 12.266 & 11.615 & 11.532 & 4575 & 1.70 & -1.30 & 2.00 & -194.0 \\
$18124339-3635339$ & 0.131 & 12.226 & 11.600 & 11.466 & 4525 & 1.65 & -1.28 & 1.55 & +82.0 \\
$18113921-3652069$ & 0.134 & 11.927 & 11.269 & 11.114 & 4385 & 1.40 & -1.26 & 1.90 & +81.9 \\
$18111325-3646007$ & 0.134 & 12.093 & 11.427 & 11.327 & 4510 & 1.65 & -1.21 & 1.40 & -191.8 \\
$18113833-3626322$ & 0.146 & 11.951 & 11.219 & 11.077 & 4250 & 1.30 & -1.18 & 2.00 & -154.2 \\
$18111018-3639350$ & 0.140 & 11.618 & 10.897 & 10.775 & 4320 & 1.35 & -1.16 & 1.40 & -184.0 \\
$18135243-3630481$ & 0.123 & 11.896 & 11.285 & 11.154 & 4560 & 1.75 & -1.15 & 1.60 & -57.0 \\
$18121150-3627559$ & 0.143 & 12.015 & 11.351 & 11.214 & 4430 & 1.60 & -1.10 & 1.50 & -101.7 \\
$18111963-3647245$ & 0.134 & 11.955 & 11.279 & 11.138 & 4375 & 1.50 & -1.06 & 1.45 & -66.4 \\
$18140023-3635461$ & 0.125 & 11.715 & 11.033 & 10.892 & 4345 & 1.50 & -1.05 & 1.80 & +144.4 \\
$18135881-3639345$ & 0.128 & 11.376 & 10.692 & 10.555 & 4355 & 1.50 & -0.99 & 1.70 & -102.6 \\
$18121436-3654551$ & 0.143 & 11.964 & 11.281 & 11.109 & 4290 & 1.50 & -0.93 & 1.75 & +67.0 \\
$18124228-3655079$ & 0.138 & 11.062 & 10.336 & 10.178 & 4215 & 1.35 & -0.92 & 1.95 & +4.7 \\
$18122530-3637366$ & 0.131 & 11.520 & 10.795 & 10.661 & 4265 & 1.50 & -0.89 & 1.60 & -4.8 \\
$18131370-3651584$ & 0.129 & 11.994 & 11.321 & 11.189 & 4400 & 1.55 & -0.88 & 1.20 & -54.7 \\
$18134668-3623541$ & 0.118 & 12.194 & 11.482 & 11.378 & 4355 & 1.60 & -0.88 & 1.80 & -0.5 \\
$18105081-3642137$ & 0.135 & 11.853 & 11.227 & 11.098 & 4545 & 1.75 & -0.87 & 1.10 & -149.6 \\
$18124219-3631172$ & 0.136 & 11.829 & 11.144 & 10.988 & 4315 & 1.55 & -0.87 & 1.60 & +30.8 \\
$18133688-3647054$ & 0.125 & 11.510 & 10.860 & 10.739 & 4485 & 1.70 & -0.86 & 1.70 & -41.8 \\
$18123142-3639294$ & 0.144 & 11.933 & 11.189 & 11.032 & 4185 & 1.45 & -0.80 & 1.75 & +255.1 \\
$18133038-3632229$ & 0.131 & 12.339 & 11.734 & 11.621 & 4645 & 1.90 & -0.80 & 1.80 & -25.6 \\
$18133292-3624179$ & 0.121 & 12.425 & 11.789 & 11.723 & 4675 & 1.95 & -0.78 & 2.00 & +27.7 \\
$18111681-3636318$ & 0.139 & 9.774 & 9.033 & 8.866 & 4165 & 1.25 & -0.77 & 1.40 & +73.0 \\
& & & & & & & & & +1.9 \\
\end{tabular}


TABLE 2-Continued

\begin{tabular}{|c|c|c|c|c|c|c|c|c|c|}
\hline $\begin{array}{l}\text { Star ID } \\
\text { 2MASS }\end{array}$ & $\mathrm{E}(\mathrm{B}-\mathrm{V})$ & $\mathrm{J}$ & $\mathrm{H}$ & $\mathrm{K}_{\mathrm{S}}$ & $\begin{array}{l}\mathrm{T}_{\text {eff }} \\
(\mathrm{K})\end{array}$ & $\begin{array}{l}\log (g) \\
(\mathrm{cgs})\end{array}$ & {$[\mathrm{Fe} / \mathrm{H}]$} & $\begin{array}{c}\mathrm{V}_{\mathrm{t}} \\
\left(\mathrm{km} \mathrm{s}^{-1}\right)\end{array}$ & $\begin{array}{c}\text { RV Helio. } \\
\left(\mathrm{km} \mathrm{s}^{-1}\right)\end{array}$ \\
\hline $18105004-3640063$ & 0.139 & 12.215 & 11.510 & 11.438 & 4490 & 1.70 & -0.76 & 1.80 & -106.6 \\
\hline $18112334-3634194$ & 0.139 & 11.853 & 11.216 & 11.072 & 4475 & 1.80 & -0.74 & 1.50 & +56.6 \\
\hline $18131027-3632343$ & 0.133 & 11.756 & 11.061 & 10.917 & 4320 & 1.60 & -0.73 & 1.60 & +103.3 \\
\hline $18110293-3628041$ & 0.139 & 11.708 & 11.001 & 10.823 & 4215 & 1.35 & -0.72 & 1.95 & +9.3 \\
\hline $18132040-3623075$ & 0.128 & 10.612 & 9.934 & 9.802 & 4385 & 1.70 & -0.71 & 1.65 & -68.2 \\
\hline $18135588-3629188$ & 0.120 & 11.675 & 11.027 & 10.896 & 4455 & 1.75 & -0.71 & 1.95 & -83.0 \\
\hline $18105075-3635061$ & 0.139 & 11.420 & 10.656 & 10.526 & 4195 & 1.40 & -0.70 & 1.40 & -56.2 \\
\hline $18104188-3634541$ & 0.141 & 11.795 & 11.139 & 11.067 & 4630 & 1.90 & -0.68 & 1.80 & -73.4 \\
\hline $18132998-3628578$ & 0.125 & 11.067 & 10.325 & 10.169 & 4170 & 1.40 & -0.68 & 1.85 & +27.6 \\
\hline $18130544-3616563$ & 0.136 & 12.015 & 11.309 & 11.145 & 4245 & 1.55 & -0.66 & 1.70 & -63.3 \\
\hline $18115908-3654510$ & 0.142 & 11.512 & 10.787 & 10.629 & 4225 & 1.50 & -0.64 & 1.35 & -124.5 \\
\hline $18121077-3639094$ & 0.135 & 12.089 & 11.423 & 11.245 & 4305 & 1.65 & -0.63 & 1.70 & -57.7 \\
\hline $18120614-3629571$ & 0.142 & 11.505 & 10.825 & 10.702 & 4425 & 1.75 & -0.58 & 1.35 & +29.0 \\
\hline $18120213-3641599$ & 0.141 & 11.866 & 11.297 & 11.126 & 4595 & 1.90 & -0.55 & 1.55 & -30.7 \\
\hline $18123350-3654398$ & 0.141 & 11.891 & 11.155 & 10.993 & 4185 & 1.55 & -0.54 & 1.70 & -96.9 \\
\hline $18120973-3654184$ & 0.143 & 11.594 & 10.887 & 10.663 & 4115 & 1.40 & -0.53 & 2.00 & -91.9 \\
\hline $18105641-3632359$ & 0.139 & 11.834 & 11.178 & 11.017 & 4380 & 1.70 & -0.52 & 1.55 & -269.5 \\
\hline $18125106-3654091$ & 0.134 & 11.873 & 11.257 & 11.135 & 4590 & 1.90 & -0.51 & 1.20 & +24.7 \\
\hline $18111581-3648469$ & 0.132 & 11.879 & 11.123 & 10.973 & 4160 & 1.55 & -0.51 & 1.40 & +106.2 \\
\hline $18131387-3630130$ & 0.132 & 12.065 & 11.373 & 11.201 & 4255 & 1.55 & -0.51 & 1.70 & -202.0 \\
\hline $18123592-3634476$ & 0.131 & 11.227 & 10.485 & 10.315 & 4145 & 1.55 & -0.50 & 1.85 & -139.1 \\
\hline $18135736-3638266$ & 0.129 & 12.042 & 11.379 & 11.178 & 4250 & 1.60 & -0.49 & 1.40 & -1.1 \\
\hline $18133233-3626133$ & 0.121 & 11.860 & 11.167 & 10.955 & 4145 & 1.55 & -0.49 & 1.60 & -207.5 \\
\hline $18125003-3630269$ & 0.137 & 12.146 & 11.496 & 11.343 & 4415 & 1.75 & -0.49 & 1.95 & -48.7 \\
\hline
\end{tabular}


TABLE 2-Continued

\begin{tabular}{cccccccccc}
\hline \hline $\begin{array}{c}\text { Star ID } \\
\text { 2MASS }\end{array}$ & $\mathrm{E}(\mathrm{B}-\mathrm{V})$ & $\mathrm{J}$ & $\mathrm{H}$ & $\mathrm{K}_{\mathrm{S}}$ & $\begin{array}{c}\mathrm{T}_{\text {eff }} \\
(\mathrm{K})\end{array}$ & $\begin{array}{c}\log (\mathrm{g}) \\
(\mathrm{cgs})\end{array}$ & {$[\mathrm{Fe} / \mathrm{H}]$} & $\begin{array}{c}\mathrm{V}_{\mathrm{t}} \\
\left(\mathrm{km} \mathrm{s}^{-1}\right)\end{array}$ & $\begin{array}{c}\text { RV Helio. } \\
\left(\mathrm{km} \mathrm{s}^{-1}\right)\end{array}$ \\
\hline $18130723-3618385$ & 0.131 & 12.187 & 11.577 & 11.418 & 4500 & 1.80 & -0.45 & 1.60 & -53.2 \\
$18125800-3616427$ & 0.138 & 12.427 & 11.791 & 11.716 & 4675 & 2.05 & -0.44 & 1.60 & +59.9 \\
$18111323-3637030$ & 0.139 & 12.128 & 11.466 & 11.356 & 4500 & 1.80 & -0.44 & 1.30 & +7.6 \\
$18130427-3630012$ & 0.135 & 12.363 & 11.842 & 11.637 & 4630 & 2.00 & -0.44 & 1.40 & +5.1 \\
$18124614-3617326$ & 0.143 & 11.966 & 11.263 & 11.093 & 4250 & 1.60 & -0.43 & 1.70 & -36.3 \\
$18120399-3615387$ & 0.156 & 11.779 & 11.018 & 10.891 & 4230 & 1.60 & -0.43 & 1.20 & -111.6 \\
$18123475-3624478$ & 0.143 & 12.094 & 11.408 & 11.305 & 4460 & 1.85 & -0.43 & 1.80 & -104.4 \\
$18115661-3617533$ & 0.156 & 12.210 & 11.564 & 11.459 & 4590 & 1.95 & -0.41 & 1.75 & +21.3 \\
$18113299-3623341$ & 0.147 & 11.235 & 10.514 & 10.369 & 4270 & 1.65 & -0.40 & 1.35 & -64.8 \\
$18111553-3628156$ & 0.139 & 11.035 & 10.342 & 10.147 & 4210 & 1.60 & -0.37 & 1.60 & -90.1 \\
$18125907-3644275$ & 0.153 & 12.126 & 11.565 & 11.405 & 4670 & 2.05 & -0.37 & 1.90 & +6.9 \\
$18111726-3620302$ & 0.143 & 12.174 & 11.509 & 11.364 & 4405 & 1.80 & -0.34 & 1.95 & -21.1 \\
$18111579-3625114$ & 0.141 & 11.649 & 10.881 & 10.733 & 4145 & 1.55 & -0.34 & 1.25 & -6.3 \\
$18131305-3650449$ & 0.128 & 11.938 & 11.356 & 11.169 & 4495 & 1.90 & -0.33 & 1.50 & -65.1 \\
$18123152-3636498$ & 0.130 & 11.523 & 10.830 & 10.618 & 4160 & 1.60 & -0.33 & 1.40 & -5.4 \\
$18125914-3619208$ & 0.140 & 11.864 & 11.262 & 11.097 & 4515 & 1.90 & -0.30 & 1.55 & -81.5 \\
$18115073-3650493$ & 0.135 & 11.856 & 11.258 & 11.053 & 4410 & 1.80 & -0.29 & 2.10 & -70.8 \\
$18114648-3625169$ & 0.150 & 12.413 & 11.796 & 11.698 & 4685 & 2.05 & -0.28 & 1.50 & -57.3 \\
$18113751-3629139$ & 0.141 & 11.649 & 10.895 & 10.724 & 4130 & 1.60 & -0.28 & 1.30 & -52.0 \\
$18122243-3637539$ & 0.133 & 11.362 & 10.715 & 10.546 & 4375 & 1.80 & -0.24 & 1.60 & +44.1 \\
$18133366-3644575$ & 0.127 & 12.331 & 11.752 & 11.627 & 4680 & 2.05 & -0.23 & 1.70 & -79.5 \\
$18134702-3641477$ & 0.126 & 12.083 & 11.512 & 11.376 & 4670 & 2.05 & -0.23 & 1.60 & +99.3 \\
$18125228-3618294$ & 0.140 & 11.766 & 11.150 & 11.028 & 4600 & 1.95 & -0.21 & 1.65 & +7.6 \\
$18110942-3647198$ & 0.133 & 12.288 & 11.702 & 11.573 & 4660 & 2.00 & -0.21 & 1.70 & -49.3
\end{tabular}


TABLE 2-Continued

\begin{tabular}{cccccccccc}
\hline \hline Star ID & $\mathrm{E}(\mathrm{B}-\mathrm{V})$ & $\mathrm{J}$ & $\mathrm{H}$ & $\mathrm{K}_{\mathrm{S}}$ & $\begin{array}{c}\mathrm{T}_{\text {eff }} \\
(\mathrm{K})\end{array}$ & $\begin{array}{c}\log (\mathrm{g}) \\
(\mathrm{cgs})\end{array}$ & {$[\mathrm{Fe} / \mathrm{H}]$} & $\begin{array}{c}\mathrm{V}_{\mathrm{t}} \\
\left(\mathrm{km} \mathrm{s}^{-1}\right)\end{array}$ & $\begin{array}{c}\text { RV Helio. } \\
\left(\mathrm{km} \mathrm{s}^{-1}\right)\end{array}$ \\
\hline $18104824-3638507$ & 0.138 & 11.957 & 11.208 & 11.060 & 4185 & 1.60 & -0.19 & 1.20 & -224.2 \\
$18122301-3629462$ & 0.137 & 10.311 & 9.598 & 9.392 & 4135 & 1.60 & -0.19 & 1.65 & +28.2 \\
$18133332-3645578$ & 0.125 & 11.930 & 11.194 & 11.015 & 4130 & 1.60 & -0.18 & 1.70 & +23.5 \\
$18123434-3652241$ & 0.137 & 10.907 & 10.251 & 10.081 & 4355 & 1.75 & -0.18 & 1.65 & -142.6 \\
$18112311-3625456$ & 0.143 & 11.690 & 11.078 & 10.924 & 4525 & 1.95 & -0.17 & 2.00 & -0.9 \\
$18125463-3649277$ & 0.137 & 11.588 & 10.938 & 10.756 & 4340 & 1.75 & -0.14 & 1.65 & -103.3 \\
$18123925-3620465$ & 0.143 & 11.736 & 11.001 & 10.840 & 4195 & 1.65 & -0.14 & 1.30 & -177.4 \\
$18110629-3632146$ & 0.137 & 10.483 & 9.850 & 9.628 & 4285 & 1.70 & -0.13 & 1.70 & +26.1 \\
$18133687-3639590$ & 0.135 & 12.001 & 11.399 & 11.274 & 4625 & 2.00 & -0.11 & 1.90 & -81.2 \\
$18111505-3620576$ & 0.142 & 11.836 & 11.238 & 11.097 & 4600 & 2.00 & -0.09 & 1.40 & -69.9 \\
$18120832-3628243$ & 0.143 & 10.574 & 9.910 & 9.730 & 4320 & 1.75 & -0.09 & 1.55 & +39.9 \\
$18130815-3631460$ & 0.133 & 10.652 & 9.901 & 9.723 & 4110 & 1.55 & -0.09 & 1.25 & -70.8 \\
$18120407-3636481$ & 0.134 & 11.401 & 10.686 & 10.508 & 4190 & 1.65 & -0.09 & 1.40 & +82.4 \\
$18135786-3640300$ & 0.128 & 11.801 & 11.147 & 10.948 & 4275 & 1.42 & -0.05 & 1.40 & -110.4 \\
$18131228-3619138$ & 0.131 & 11.336 & 10.716 & 10.525 & 4385 & 1.80 & -0.04 & 1.65 & +2.7 \\
$18113372-3650200$ & 0.134 & 11.714 & 11.085 & 10.835 & 4220 & 1.65 & -0.04 & 1.65 & +10.5 \\
$18115904-3650428$ & 0.137 & 11.481 & 10.791 & 10.584 & 4185 & 1.65 & -0.01 & 1.40 & -24.8 \\
$18115448-3641161$ & 0.139 & 11.705 & 11.047 & 10.904 & 4425 & 1.85 & -0.01 & 1.75 & -79.7 \\
$18123678-3649019$ & 0.143 & 11.278 & 10.691 & 10.493 & 4470 & 1.85 & +0.02 & 1.30 & -104.1 \\
$18113965-3645408$ & 0.135 & 11.884 & 11.193 & 11.032 & 4290 & 1.80 & +0.05 & 1.30 & -15.0 \\
$18135362-3636538$ & 0.129 & 11.615 & 11.002 & 10.836 & 4465 & 2.00 & +0.06 & 1.85 & -69.2 \\
$18123168-3631555$ & 0.133 & 12.427 & 11.801 & 11.705 & 4635 & 2.05 & +0.09 & 1.80 & +57.4 \\
$18115398-3623290$ & 0.154 & 11.588 & 10.842 & 10.653 & 4120 & 1.70 & +0.09 & 1.60 & -96.1 \\
$18123393-3615508$ & 0.149 & 11.863 & 11.301 & 11.133 & 4640 & 2.05 & +0.11 & 1.75 & -19.9
\end{tabular}


TABLE 2-Continued

\begin{tabular}{|c|c|c|c|c|c|c|c|c|c|}
\hline $\begin{array}{l}\text { Star ID } \\
\text { 2MASS }\end{array}$ & $\mathrm{E}(\mathrm{B}-\mathrm{V})$ & $\mathrm{J}$ & $\mathrm{H}$ & $\mathrm{K}_{\mathrm{S}}$ & $\begin{array}{l}\mathrm{T}_{\text {eff }} \\
(\mathrm{K})\end{array}$ & $\begin{array}{c}\log (g) \\
(\operatorname{cgs})\end{array}$ & {$[\mathrm{Fe} / \mathrm{H}]$} & $\begin{array}{c}\mathrm{V}_{\mathrm{t}} \\
\left(\mathrm{km} \mathrm{s}^{-1}\right)\end{array}$ & $\begin{array}{l}\text { RV Helio. } \\
\left(\mathrm{km} \mathrm{s}^{-1}\right)\end{array}$ \\
\hline $18120689-3643231$ & 0.143 & 11.523 & 10.920 & 10.767 & 4555 & 2.00 & +0.11 & 1.80 & -70.7 \\
\hline $18130482-3649019$ & 0.135 & 11.849 & 11.118 & 10.935 & 4145 & 1.70 & +0.13 & 1.80 & -116.7 \\
\hline $18110352-3646275$ & 0.134 & 10.786 & 10.068 & 9.880 & 4160 & 1.60 & +0.14 & 1.50 & -77.8 \\
\hline $18105013-3629557$ & 0.143 & 10.701 & 10.026 & 9.868 & 4345 & 1.85 & +0.17 & 1.30 & +14.2 \\
\hline $18121897-3630243$ & 0.135 & 12.261 & 11.645 & 11.537 & 4635 & 2.05 & +0.22 & 1.50 & -28.7 \\
\hline $18122029-3652333$ & 0.139 & 10.984 & 10.385 & 10.190 & 4440 & 1.90 & +0.26 & 1.60 & -42.1 \\
\hline \multicolumn{10}{|c|}{$(\mathrm{l}, \mathrm{b})=(+8.5,+9)$} \\
\hline $17311256-1702018$ & 0.359 & 10.392 & 9.555 & 9.385 & 4215 & 1.10 & -1.26 & 1.70 & +12.9 \\
\hline $17290874-1632431$ & 0.374 & 10.600 & 9.753 & 9.544 & 4125 & 0.95 & -1.23 & 1.90 & +35.7 \\
\hline $17302885-1639588$ & 0.390 & 10.855 & 10.048 & 9.824 & 4200 & 1.15 & -1.17 & 1.90 & -167.8 \\
\hline 17304789-1623192 & 0.402 & 11.235 & 10.462 & 10.213 & 4235 & 1.20 & -1.14 & 1.80 & -211.7 \\
\hline 17321080-1641479 & 0.393 & 10.232 & 9.304 & 9.117 & 4020 & 0.90 & -1.09 & 1.75 & -114.2 \\
\hline 17303589-1638299 & 0.392 & 10.631 & 9.804 & 9.603 & 4210 & 1.25 & -0.97 & 1.55 & -104.1 \\
\hline $17290513-1630383$ & 0.380 & 10.960 & 10.090 & 9.880 & 4080 & 1.10 & -0.96 & 2.15 & -63.9 \\
\hline 17314699-1654460 & 0.342 & 11.270 & 10.457 & 10.273 & 4215 & 1.35 & -0.93 & 1.60 & -93.5 \\
\hline 17305076-1615216 & 0.378 & 11.238 & 10.420 & 10.218 & 4210 & 1.30 & -0.81 & 1.70 & -196.1 \\
\hline $17323324-1627474$ & 0.438 & 10.835 & 9.934 & 9.687 & 4005 & 1.05 & -0.80 & 1.75 & +40.6 \\
\hline $17312795-1631423$ & 0.412 & 11.292 & 10.466 & 10.270 & 4250 & 1.45 & -0.78 & 1.75 & +70.9 \\
\hline $17290285-1638344$ & 0.383 & 11.365 & 10.605 & 10.362 & 4255 & 1.50 & -0.73 & 2.00 & -158.2 \\
\hline $17303378-1703086$ & 0.364 & 11.011 & 10.118 & 9.906 & 4010 & 1.05 & -0.71 & 1.70 & +4.6 \\
\hline 17304223-1609274 & 0.378 & 11.242 & 10.382 & 10.177 & 4110 & 1.35 & -0.64 & 1.65 & +78.8 \\
\hline 17304908-1633101 & 0.400 & 10.667 & 9.831 & 9.605 & 4140 & 1.35 & -0.64 & 1.95 & -25.7 \\
\hline 17291459-1650085 & 0.394 & 10.864 & 9.974 & 9.778 & 4080 & 1.30 & -0.62 & 1.80 & +98.1 \\
\hline $17300674-1638541$ & 0.382 & 10.903 & 10.035 & 9.823 & 4080 & 1.30 & -0.57 & 1.65 & -40.1 \\
\hline
\end{tabular}


TABLE 2-Continued

\begin{tabular}{|c|c|c|c|c|c|c|c|c|c|}
\hline $\begin{array}{l}\text { Star ID } \\
2 \mathrm{MASS}\end{array}$ & $\mathrm{E}(\mathrm{B}-\mathrm{V})$ & $\mathrm{J}$ & $\mathrm{H}$ & $\mathrm{K}_{\mathrm{S}}$ & $\begin{array}{l}\mathrm{T}_{\text {eff }} \\
(\mathrm{K})\end{array}$ & $\begin{array}{c}\log (g) \\
(\operatorname{cgs})\end{array}$ & {$[\mathrm{Fe} / \mathrm{H}]$} & $\begin{array}{c}\mathrm{V}_{\mathrm{t}} \\
\left(\mathrm{km} \mathrm{s}^{-1}\right)\end{array}$ & $\begin{array}{l}\text { RV Helio. } \\
\left(\mathrm{km} \mathrm{s}^{-1}\right)\end{array}$ \\
\hline $17315010-1653413$ & 0.336 & 11.523 & 10.706 & 10.542 & 4245 & 1.60 & -0.55 & 1.75 & +37.4 \\
\hline $17295493-1613273$ & 0.375 & 11.251 & 10.391 & 10.179 & 4090 & 1.25 & -0.54 & 1.50 & -66.8 \\
\hline $17311523-1618542$ & 0.396 & 11.326 & 10.540 & 10.312 & 4245 & 1.60 & -0.52 & 1.50 & +183.9 \\
\hline $17314811-1621558$ & 0.425 & 10.998 & 10.134 & 9.927 & 4150 & 1.45 & -0.52 & 1.85 & -39.0 \\
\hline $17304861-1646459$ & 0.350 & 11.023 & 10.154 & 9.927 & 4010 & 1.25 & -0.51 & 1.60 & +73.5 \\
\hline $17323959-1625298$ & 0.439 & 11.178 & 10.363 & 10.114 & 4185 & 1.55 & -0.50 & 1.65 & -24.6 \\
\hline $17285711-1633566$ & 0.385 & 10.951 & 10.171 & 9.901 & 4150 & 1.45 & -0.50 & 1.55 & +24.5 \\
\hline $17314673-1641228$ & 0.382 & 10.655 & 9.813 & 9.553 & 4035 & 1.25 & -0.48 & 1.90 & +57.1 \\
\hline $17294476-1626118$ & 0.357 & 10.946 & 10.064 & 9.846 & 4010 & 1.30 & -0.44 & 2.10 & -139.1 \\
\hline $17320650-1643389$ & 0.394 & 10.740 & 9.880 & 9.620 & 4010 & 1.15 & -0.39 & 1.65 & +52.8 \\
\hline $17314427-1640265$ & 0.381 & 11.119 & 10.307 & 10.101 & 4215 & 1.65 & -0.39 & 1.75 & -96.9 \\
\hline $17300064-1701075$ & 0.360 & 11.003 & 10.168 & 9.900 & 4005 & 1.25 & -0.38 & 1.50 & -28.0 \\
\hline $17302935-1652277$ & 0.361 & 11.246 & 10.494 & 10.245 & 4230 & 1.60 & -0.34 & 1.70 & +17.7 \\
\hline $17320652-1619563$ & 0.421 & 11.036 & 10.162 & 9.950 & 4115 & 1.50 & -0.30 & 1.35 & +49.9 \\
\hline $17304464-1631471$ & 0.400 & 11.327 & 10.510 & 10.266 & 4145 & 1.60 & -0.29 & 1.70 & +76.1 \\
\hline $17321715-1632467$ & 0.399 & 10.671 & 9.835 & 9.616 & 4155 & 1.55 & -0.28 & 1.70 & -27.2 \\
\hline $17321969-1653156$ & 0.369 & 10.701 & 9.848 & 9.632 & 4090 & 1.45 & -0.25 & 1.40 & +100.2 \\
\hline $17315690-1630315$ & 0.412 & 11.076 & 10.212 & 9.989 & 4100 & 1.55 & -0.20 & 1.60 & +35.8 \\
\hline $17312870-1619572$ & 0.412 & 10.910 & 10.075 & 9.779 & 4010 & 1.40 & -0.19 & 1.55 & +17.7 \\
\hline $17294541-1649018$ & 0.422 & 10.704 & 9.821 & 9.582 & 4045 & 1.40 & -0.19 & 1.50 & -73.8 \\
\hline $17305067-1644006$ & 0.367 & 11.261 & 10.377 & 10.165 & 4030 & 1.45 & -0.19 & 1.65 & +21.8 \\
\hline $17312457-1652524$ & 0.327 & 11.331 & 10.548 & 10.324 & 4175 & 1.65 & -0.18 & 1.55 & +17.4 \\
\hline $17292760-1636235$ & 0.368 & 11.239 & 10.401 & 10.178 & 4105 & 1.60 & -0.18 & 1.40 & +95.9 \\
\hline $17295978-1633590$ & 0.361 & 11.161 & 10.316 & 10.077 & 4050 & 1.50 & -0.17 & 1.55 & -76.3 \\
\hline
\end{tabular}


TABLE 2-Continued

\begin{tabular}{|c|c|c|c|c|c|c|c|c|c|}
\hline $\begin{array}{l}\text { Star ID } \\
\text { 2MASS }\end{array}$ & $\mathrm{E}(\mathrm{B}-\mathrm{V})$ & $\mathrm{J}$ & $\mathrm{H}$ & $\mathrm{K}_{\mathrm{S}}$ & $\begin{array}{l}\mathrm{T}_{\text {eff }} \\
(\mathrm{K})\end{array}$ & $\begin{array}{l}\log (g) \\
(\mathrm{cgs})\end{array}$ & {$[\mathrm{Fe} / \mathrm{H}]$} & $\begin{array}{c}\mathrm{V}_{\mathrm{t}} \\
\left(\mathrm{km} \mathrm{s}^{-1}\right)\end{array}$ & $\begin{array}{c}\text { RV Helio. } \\
\left(\mathrm{km} \mathrm{s}^{-1}\right)\end{array}$ \\
\hline $17322323-1649335$ & 0.401 & 11.385 & 10.553 & 10.343 & 4185 & 1.65 & -0.15 & 1.75 & +65.1 \\
\hline $17321117-1654590$ & 0.356 & 10.419 & 9.638 & 9.396 & 4175 & 1.65 & -0.13 & 1.65 & -5.7 \\
\hline $17313606-1607539$ & 0.384 & 11.481 & 10.727 & 10.466 & 4230 & 1.65 & -0.11 & 1.75 & +142.4 \\
\hline $17295331-1653069$ & 0.405 & 10.744 & 9.925 & 9.695 & 4175 & 1.65 & -0.10 & 1.35 & -125.7 \\
\hline $17302818-1636032$ & 0.388 & 10.680 & 9.901 & 9.638 & 4170 & 1.60 & -0.07 & 1.40 & +42.3 \\
\hline $17293118-1638081$ & 0.371 & 10.679 & 9.901 & 9.654 & 4190 & 1.70 & -0.07 & 2.00 & -29.2 \\
\hline $17291994-1618302$ & 0.391 & 10.640 & 9.807 & 9.526 & 4020 & 1.45 & -0.07 & 1.95 & +61.4 \\
\hline $17320457-1646391$ & 0.392 & 11.037 & 10.217 & 9.960 & 4100 & 1.60 & -0.05 & 1.70 & +42.3 \\
\hline $17310747-1620049$ & 0.401 & 11.032 & 10.233 & 9.989 & 4185 & 1.65 & -0.04 & 1.50 & +80.7 \\
\hline $17323392-1636330$ & 0.403 & 11.056 & 10.172 & 9.942 & 4035 & 1.55 & -0.04 & 1.30 & +133.3 \\
\hline $17312572-1609092$ & 0.388 & 10.768 & 10.001 & 9.755 & 4240 & 1.65 & -0.03 & 1.65 & +14.6 \\
\hline $17300015-1609464$ & 0.386 & 11.432 & 10.622 & 10.405 & 4205 & 1.65 & +0.03 & 1.60 & -53.4 \\
\hline $17305894-1637056$ & 0.395 & 10.582 & 9.716 & 9.467 & 4020 & 1.50 & +0.10 & 1.30 & -46.7 \\
\hline $17291621-1647189$ & 0.412 & 10.739 & 9.855 & 9.611 & 4015 & 1.50 & +0.12 & 1.25 & -52.3 \\
\hline $17302194-1643531$ & 0.388 & 10.522 & 9.638 & 9.427 & 4055 & 1.55 & +0.14 & 1.30 & +43.6 \\
\hline $17311959-1643577$ & 0.349 & 10.989 & 10.203 & 9.947 & 4125 & 1.65 & +0.16 & 1.60 & -25.5 \\
\hline $17311229-1659047$ & 0.350 & 11.011 & 10.168 & 9.919 & 4020 & 1.55 & +0.20 & 1.20 & -90.2 \\
\hline $17313313-1659216$ & 0.355 & 10.843 & 10.098 & 9.830 & 4195 & 1.80 & +0.21 & 1.50 & +45.0 \\
\hline $17301749-1629529$ & 0.376 & 10.588 & 9.819 & 9.543 & 4150 & 1.70 & +0.23 & 1.50 & +68.8 \\
\hline $17294261-1622440$ & 0.353 & 11.368 & 10.533 & 10.316 & 4105 & 1.65 & +0.23 & 1.25 & -101.0 \\
\hline $17323625-1637551$ & 0.402 & 10.360 & 9.557 & 9.336 & 4230 & 1.80 & +0.26 & 1.45 & +53.2 \\
\hline $17314292-1620036$ & 0.419 & 10.933 & 10.095 & 9.817 & 4050 & 1.60 & +0.28 & 1.60 & -37.6 \\
\hline $17310955-1635152$ & 0.397 & 10.717 & 9.870 & 9.612 & 4045 & 1.55 & +0.32 & 1.40 & +37.0 \\
\hline $17304312-1630318$ & 0.396 & 11.403 & 10.624 & 10.369 & 4200 & 1.75 & +0.37 & 1.55 & -31.3 \\
\hline
\end{tabular}


TABLE 2-Continued

\begin{tabular}{ccccccccccc}
\hline \hline $\begin{array}{l}\text { Star ID } \\
\text { 2MASS }\end{array}$ & $\mathrm{E}(\mathrm{B}-\mathrm{V})$ & $\mathrm{J}$ & $\mathrm{H}$ & $\mathrm{K}_{\mathrm{S}}$ & $\begin{array}{c}\mathrm{T}_{\text {eff }} \\
(\mathrm{K})\end{array}$ & $\begin{array}{c}\log (\mathrm{g}) \\
(\mathrm{cgs})\end{array}$ & {$[\mathrm{Fe} / \mathrm{H}]$} & $\begin{array}{c}\mathrm{V}_{\mathrm{t}} \\
\left(\mathrm{km} \mathrm{s}^{-1}\right)\end{array}$ & $\begin{array}{c}\text { RV Helio. } \\
\left(\mathrm{km} \mathrm{s}^{-1}\right)\end{array}$ \\
\hline $17285469-1627232$ & 0.394 & 10.854 & 10.085 & 9.844 & 4255 & 1.90 & +0.42 & 1.35 & +15.8 \\
$17310360-1700146$ & 0.355 & 10.861 & 10.086 & 9.798 & 4085 & 1.70 & +0.43 & 1.50 & -36.5 \\
$17295898-1652101$ & 0.396 & 11.252 & 10.477 & 10.214 & 4190 & 1.80 & +0.59 & 1.50 & +73.1 \\
\hline
\end{tabular}


TABLE 3

Chemical Abundance Ratios

\begin{tabular}{ccccc}
\hline \hline Star ID & {$[\mathrm{Fe} / \mathrm{H}]$} & {$[\mathrm{O} / \mathrm{Fe}]$} & {$[\mathrm{Si} / \mathrm{Fe}]$} & {$[\mathrm{Ca} / \mathrm{Fe}]$} \\
2MASS & & & & \\
\hline & $(\mathrm{l}, \mathrm{b})=(-5.5,-7)$ & & \\
\hline $18011989-3723462$ & -1.50 & +0.67 & +0.30 & +0.26 \\
$18003452-3729217$ & -1.40 & $\ldots$ & +0.36 & +0.30 \\
$18011692-3722508$ & -1.33 & +0.58 & +0.28 & +0.42 \\
$18005584-3739103$ & -1.31 & +0.81 & $\ldots$ & $\ldots$ \\
$18015010-3735279$ & -1.15 & +0.94 & +0.41 & +0.38 \\
$18005916-3727585$ & -1.04 & +0.79 & +0.39 & +0.46 \\
$18005234-3715478$ & -0.99 & +0.59 & +0.24 & +0.41 \\
$18005027-3707505$ & -0.99 & +0.81 & +0.31 & +0.12 \\
$18020106-3737561$ & -0.98 & +0.62 & +0.30 & +0.49 \\
$17595541-3713447$ & -0.90 & +0.72 & $\ldots$ & $\ldots$ \\
$17595664-3725304$ & -0.89 & +0.61 & +0.33 & +0.24 \\
$18021345-3730371$ & -0.80 & $\ldots$ & +0.38 & +0.43 \\
$17594241-3718247$ & -0.76 & +0.61 & +0.41 & $\ldots$ \\
$18013342-3726339$ & -0.76 & $\ldots$ & +0.31 & +0.26 \\
$18013580-3740055$ & -0.75 & +0.51 & +0.43 & +0.40 \\
$18003204-3726008$ & -0.75 & +0.88 & +0.35 & +0.44 \\
$18013799-3725131$ & -0.74 & +0.80 & +0.45 & +0.37 \\
$18022149-3713494$ & -0.71 & $\ldots$ & +0.27 & +0.09 \\
$18011408-3711299$ & -0.69 & +0.69 & +0.40 & +0.21 \\
$18021275-3737543$ & -0.67 & $\ldots$ & $\ldots$ & +0.43 \\
$17595027-3727293$ & -0.66 & $\ldots$ & +0.21 & +0.30 \\
$18002115-3715519$ & -0.61 & +0.63 & +0.27 & +0.30 \\
$17595366-3728186$ & -0.59 & $\ldots$ & +0.31 & +0.27 \\
$18013960-3723398$ & -0.58 & +0.38 & +0.31 & +0.34 \\
$18001354-3709122$ & -0.58 & +0.53 & +0.38 & +0.40 \\
$18000535-3735315$ & -0.57 & +0.40 & +0.29 & +0.25 \\
$18021862-3734204$ & -0.57 & $\ldots$ & $\ldots$ & +0.17 \\
$18013848-3721442$ & -0.55 & +0.60 & +0.30 & +0.30 \\
$18014343-3708232$ & -0.54 & +0.31 & +0.19 & +0.22 \\
$18004824-3718074$ & -0.54 & +0.34 & +0.24 & +0.26 \\
$17594792-3721422$ & -0.54 & $\ldots$ & +0.25 & +0.21
\end{tabular}


TABle 3-Continued

\begin{tabular}{ccccc}
\hline \hline Star ID & {$[\mathrm{Fe} / \mathrm{H}]$} & {$[\mathrm{O} / \mathrm{Fe}]$} & {$[\mathrm{Si} / \mathrm{Fe}]$} & {$[\mathrm{Ca} / \mathrm{Fe}]$} \\
2MASS & & & & \\
\hline $18011941-3721250$ & -0.54 & $\ldots$ & $\ldots$ & +0.27 \\
$18005852-3711524$ & -0.53 & +0.56 & +0.45 & +0.59 \\
$18020816-3714015$ & -0.53 & +0.58 & +0.29 & +0.28 \\
$18010932-3702079$ & -0.49 & +0.39 & +0.10 & +0.19 \\
$18010065-3740456$ & -0.49 & +0.74 & +0.41 & +0.29 \\
$17595640-3710383$ & -0.43 & +0.31 & +0.26 & +0.38 \\
$18012462-3738382$ & -0.43 & +0.63 & +0.26 & +0.32 \\
$18024484-3714159$ & -0.42 & +0.27 & +0.08 & +0.28 \\
$18002880-3710171$ & -0.42 & +0.32 & +0.27 & +0.34 \\
$18013206-3708133$ & -0.41 & $\ldots$ & $\ldots$ & $\ldots$ \\
$18004074-3740058$ & -0.36 & +0.13 & +0.08 & +0.34 \\
$18015431-3717070$ & -0.34 & +0.37 & +0.17 & +0.16 \\
$18000013-3715053$ & -0.34 & +0.44 & +0.19 & +0.17 \\
$18021006-3738193$ & -0.32 & +0.39 & +0.03 & +0.42 \\
$17595442-3721118$ & -0.29 & +0.29 & +0.25 & +0.19 \\
$18021721-3736096$ & -0.25 & +0.22 & -0.08 & +0.11 \\
$18005096-3733499$ & -0.25 & +0.70 & +0.38 & $\ldots$ \\
$18013395-3705551$ & -0.24 & +0.29 & +0.29 & +0.36 \\
$18011851-3706092$ & -0.24 & +0.34 & +0.24 & +0.29 \\
$18005923-3707111$ & -0.24 & $\ldots$ & +0.19 & +0.05 \\
$18022648-3728188$ & -0.20 & +0.15 & +0.04 & +0.09 \\
$18024919-3727234$ & -0.16 & +0.21 & +0.07 & +0.12 \\
$18023660-3716289$ & -0.14 & +0.29 & +0.27 & -0.04 \\
$18005395-3731231$ & -0.13 & -0.27 & +0.04 & +0.16 \\
$17595509-3711099$ & -0.13 & $\ldots$ & -0.20 & +0.13 \\
$18010322-3705447$ & -0.10 & +0.00 & +0.04 & +0.03 \\
$18014769-3739220$ & -0.10 & +0.10 & $\ldots$ & $\ldots$ \\
$17594807-3715283$ & -0.09 & +0.14 & -0.11 & +0.32 \\
$18013618-3706311$ & -0.07 & +0.25 & +0.16 & +0.01 \\
$18003751-3728200$ & -0.03 & +0.03 & -0.01 & +0.09 \\
$18004314-3730156$ & -0.03 & $\ldots$ & -0.06 & -0.01 \\
$18014303-3740054$ & -0.03 & $\ldots$ & +0.03 & +0.08
\end{tabular}


TABLE 3-Continued

\begin{tabular}{ccccc}
\hline \hline Star ID & {$[\mathrm{Fe} / \mathrm{H}]$} & {$[\mathrm{O} / \mathrm{Fe}]$} & {$[\mathrm{Si} / \mathrm{Fe}]$} & {$[\mathrm{Ca} / \mathrm{Fe}]$} \\
2MASS & & & & \\
\hline $18011170-3727118$ & -0.01 & $\ldots$ & -0.19 & -0.32 \\
$18023846-3718345$ & -0.01 & $\ldots$ & -0.24 & +0.21 \\
$18011671-3722213$ & +0.00 & +0.10 & -0.09 & $\ldots$ \\
$18005985-3734507$ & +0.02 & $\ldots$ & $\ldots$ & $\ldots$ \\
$18000430-3711051$ & +0.06 & +0.38 & +0.13 & +0.14 \\
$18003552-3729476$ & +0.06 & $\ldots$ & -0.04 & +0.00 \\
$18001817-3732243$ & +0.08 & $\ldots$ & -0.08 & +0.33 \\
$18013039-3713086$ & +0.09 & -0.09 & -0.03 & -0.21 \\
$18012564-3741052$ & +0.10 & +0.10 & -0.16 & +0.09 \\
$18025713-3718318$ & +0.10 & $\ldots$ & -0.16 & +0.12 \\
$18011599-3741385$ & +0.11 & +0.14 & +0.23 & -0.02 \\
$18012944-3719488$ & +0.12 & $\ldots$ & -0.12 & -0.03 \\
$18020361-3719086$ & +0.15 & -0.20 & +0.13 & +0.00 \\
$18000412-3717266$ & +0.17 & -0.32 & -0.16 & +0.14 \\
$18024024-3728187$ & +0.17 & -0.12 & -0.06 & -0.20 \\
$18011177-3703344$ & +0.19 & -0.09 & -0.33 & +0.09 \\
$18001410-3725181$ & +0.19 & -0.09 & -0.23 & +0.11 \\
$18020300-3719418$ & +0.21 & -0.09 & -0.20 & +0.06 \\
$18004683-3703252$ & +0.22 & +0.08 & -0.17 & +0.15 \\
$18015404-3722564$ & +0.22 & +0.38 & -0.20 & +0.04 \\
$18013974-3704068$ & +0.27 & -0.05 & -0.25 & -0.02 \\
$18020704-3708064$ & +0.27 & -0.02 & -0.24 & -0.02 \\
$18023393-3730273$ & +0.33 & $\ldots$ & $\ldots$ & $\ldots$ \\
$18001720-3708374$ & +0.42 & -0.32 & -0.52 & -0.14 \\
$18003663-3708547$ & +0.42 & $\ldots$ & -0.32 & +0.01 \\
$18002569-3729578$ & +0.50 & -0.25 & -0.57 & -0.03 \\
$17594881-3716203$ & +0.59 & +0.01 & -0.37 & +0.01 \\
$18011201-3740090$ & +0.66 & -0.26 & -0.41 & -0.23 \\
\hline & $(1, b)=(-4,-9)$ & & \\
\hline $18122552-3653049$ & -1.40 & +0.74 & +0.15 & +0.35 \\
$18120653-3654264$ & -1.38 & $\ldots$ & +0.28 & +0.40 \\
$18110179-3630394$ & -1.38 & +0.53 & +0.28 & +0.43
\end{tabular}


TABle 3-Continued

\begin{tabular}{ccccc}
\hline \hline Star ID & {$[\mathrm{Fe} / \mathrm{H}]$} & {$[\mathrm{O} / \mathrm{Fe}]$} & {$[\mathrm{Si} / \mathrm{Fe}]$} & {$[\mathrm{Ca} / \mathrm{Fe}]$} \\
2MASS & & & & \\
\hline $18124892-3626403$ & -1.33 & +0.71 & +0.28 & +0.43 \\
$18135164-3643463$ & -1.30 & +0.70 & +0.38 & +0.34 \\
$18124339-3635339$ & -1.28 & +0.75 & +0.33 & +0.44 \\
$18113921-3652069$ & -1.26 & $\ldots$ & +0.56 & +0.22 \\
$18111325-3646007$ & -1.21 & +0.61 & +0.36 & +0.51 \\
$18113833-3626322$ & -1.18 & +0.83 & +0.57 & +0.47 \\
$18111018-3639350$ & -1.16 & +0.48 & +0.29 & +0.33 \\
$18135243-3630481$ & -1.15 & +0.50 & +0.42 & +0.22 \\
$18121150-3627559$ & -1.10 & +0.65 & +0.29 & +0.22 \\
$18111963-3647245$ & -1.06 & +0.44 & +0.38 & +0.54 \\
$18140023-3635461$ & -1.05 & +0.70 & +0.82 & +0.22 \\
$18135881-3639345$ & -0.99 & +0.54 & +0.31 & +0.35 \\
$18121436-3654551$ & -0.93 & +0.71 & +0.76 & +0.40 \\
$18124228-3655079$ & -0.92 & +0.74 & +0.62 & +0.33 \\
$18122530-3637366$ & -0.89 & +0.56 & +0.54 & +0.29 \\
$18131370-3651584$ & -0.88 & $\ldots$ & +0.53 & +0.32 \\
$18134668-3623541$ & -0.88 & $\ldots$ & $\ldots$ & +0.28 \\
$18105081-3642137$ & -0.87 & +0.57 & +0.32 & +0.57 \\
$18124219-3631172$ & -0.87 & +0.57 & +0.52 & +0.41 \\
$18133688-3647054$ & -0.86 & $\ldots$ & $\ldots$ & $\ldots$ \\
$18123142-3639294$ & -0.80 & +0.75 & +0.50 & +0.47 \\
$18133038-3632229$ & -0.80 & $\ldots$ & +0.25 & $\ldots$ \\
$18133292-3624179$ & -0.78 & +0.58 & $\ldots$ & $\ldots$ \\
$18111681-3636318$ & -0.77 & +0.53 & +0.34 & +0.25 \\
$18105004-3640063$ & -0.76 & +0.46 & +0.26 & $\ldots$ \\
$18112334-3634194$ & -0.74 & +0.49 & +0.36 & +0.51 \\
$18131027-3632343$ & -0.73 & +0.54 & +0.39 & +0.38 \\
$18110293-3628041$ & -0.72 & +0.65 & +0.71 & +0.32 \\
$18132040-3623075$ & -0.71 & +0.74 & +0.38 & +0.43 \\
$18135588-3629188$ & -0.71 & +0.88 & +0.29 & +0.20 \\
$18105075-3635061$ & -0.70 & +0.55 & +0.38 & +0.33 \\
$18104188-3634541$ & -0.68 & +0.73 & +0.28 & +0.14 \\
& & & &
\end{tabular}


TABle 3-Continued

\begin{tabular}{ccccc}
\hline \hline Star ID & {$[\mathrm{Fe} / \mathrm{H}]$} & {$[\mathrm{O} / \mathrm{Fe}]$} & {$[\mathrm{Si} / \mathrm{Fe}]$} & {$[\mathrm{Ca} / \mathrm{Fe}]$} \\
2MASS & & & & \\
\hline $18132998-3628578$ & -0.68 & +0.72 & +0.33 & +0.19 \\
$18130544-3616563$ & -0.66 & $\ldots$ & +0.50 & +0.48 \\
$18115908-3654510$ & -0.64 & +0.49 & +0.48 & +0.30 \\
$18121077-3639094$ & -0.63 & +0.53 & +0.38 & +0.11 \\
$18120614-3629571$ & -0.58 & +0.23 & +0.38 & +0.18 \\
$18120213-3641599$ & -0.55 & $\cdots$ & +0.47 & +0.26 \\
$18123350-3654398$ & -0.54 & $\ldots$ & +0.70 & +0.42 \\
$18120973-3654184$ & -0.53 & +0.78 & +0.78 & +0.17 \\
$18105641-3632359$ & -0.52 & +0.32 & +0.52 & +0.38 \\
$18125106-3654091$ & -0.51 & +0.26 & +0.21 & +0.21 \\
$18111581-3648469$ & -0.51 & +0.28 & +0.40 & +0.36 \\
$18131387-3630130$ & -0.51 & $\cdots$ & +0.25 & +0.24 \\
$18123592-3634476$ & -0.50 & +0.52 & +0.28 & +0.17 \\
$18135736-3638266$ & -0.49 & +0.24 & +0.24 & +0.39 \\
$18133233-3626133$ & -0.49 & +0.48 & +0.21 & +0.19 \\
$18125003-3630269$ & -0.49 & +0.29 & +0.49 & +0.16 \\
$18130723-3618385$ & -0.45 & +0.55 & +0.42 & +0.40 \\
$18125800-3616427$ & -0.44 & +0.34 & +0.13 & +0.45 \\
$18111323-3637030$ & -0.44 & +0.04 & +0.13 & +0.29 \\
$18130427-3630012$ & -0.44 & +0.09 & +0.34 & +0.14 \\
$18124614-3617326$ & -0.43 & $\cdots$ & +0.45 & +0.07 \\
$18120399-3615387$ & -0.43 & +0.28 & +0.12 & +0.21 \\
$18123475-3624478$ & -0.43 & +0.53 & +0.33 & +0.31 \\
$18115661-3617533$ & -0.41 & +0.11 & +0.32 & +0.55 \\
$18113299-3623341$ & -0.40 & +0.31 & +0.32 & +0.41 \\
$18111553-3628156$ & -0.37 & +0.62 & +0.35 & +0.31 \\
$18125907-3644275$ & -0.37 & +0.07 & +0.25 & +0.03 \\
$18111726-3620302$ & -0.34 & +0.26 & $\ldots$ & +0.22 \\
$18111579-3625114$ & -0.34 & +0.43 & +0.29 & +0.54 \\
$18131305-3650449$ & -0.33 & $\cdots$ & +0.34 & +0.25 \\
$18123152-3636498$ & -0.33 & +0.28 & +0.35 & +0.37 \\
$18125914-3619208$ & -0.30 & +0.27 & +0.21 & +0.30
\end{tabular}


TABle 3-Continued

\begin{tabular}{ccccc}
\hline \hline Star ID & {$[\mathrm{Fe} / \mathrm{H}]$} & {$[\mathrm{O} / \mathrm{Fe}]$} & {$[\mathrm{Si} / \mathrm{Fe}]$} & {$[\mathrm{Ca} / \mathrm{Fe}]$} \\
2MASS & & & & \\
\hline $18115073-3650493$ & -0.29 & $\ldots$ & +0.33 & +0.20 \\
$18114648-3625169$ & -0.28 & $\ldots$ & +0.41 & +0.18 \\
$18113751-3629139$ & -0.28 & $\ldots$ & +0.41 & +0.38 \\
$18122243-3637539$ & -0.24 & +0.30 & +0.13 & +0.30 \\
$18133366-3644575$ & -0.23 & +0.25 & +0.28 & $\ldots$ \\
$18134702-3641477$ & -0.23 & +0.33 & +0.41 & +0.28 \\
$18125228-3618294$ & -0.21 & -0.14 & +0.11 & +0.31 \\
$18110942-3647198$ & -0.21 & $\ldots$ & +0.06 & -0.08 \\
$18104824-3638507$ & -0.19 & +0.09 & +0.09 & +0.22 \\
$18122301-3629462$ & -0.19 & +0.13 & +0.11 & +0.14 \\
$18133332-3645578$ & -0.18 & +0.28 & +0.30 & +0.23 \\
$18123434-3652241$ & -0.18 & +0.39 & +0.24 & +0.10 \\
$18112311-3625456$ & -0.17 & -0.01 & +0.12 & +0.26 \\
$18125463-3649277$ & -0.14 & +0.54 & +0.20 & +0.16 \\
$18123925-3620465$ & -0.14 & $\ldots$ & +0.02 & +0.18 \\
$18110629-3632146$ & -0.13 & -0.07 & +0.05 & +0.07 \\
$18133687-3639590$ & -0.11 & +0.21 & +0.19 & +0.39 \\
$18111505-3620576$ & -0.09 & -0.06 & +0.01 & +0.33 \\
$18120832-3628243$ & -0.09 & +0.15 & +0.24 & +0.28 \\
$18130815-3631460$ & -0.09 & +0.14 & +0.17 & +0.27 \\
$18120407-3636481$ & -0.09 & +0.29 & +0.06 & +0.14 \\
$18135786-3640300$ & -0.05 & -0.10 & -0.05 & +0.12 \\
$18131228-3619138$ & -0.04 & -0.21 & +0.05 & +0.22 \\
$18113372-3650200$ & -0.04 & -0.11 & $\ldots$ & +0.03 \\
$18115904-3650428$ & -0.01 & $\ldots$ & +0.06 & +0.00 \\
$18115448-3641161$ & -0.01 & +0.05 & +0.04 & -0.04 \\
$18123678-3649019$ & +0.02 & +0.33 & -0.12 & +0.20 \\
$18113965-3645408$ & +0.05 & $\ldots$ & -0.40 & -0.07 \\
$18135362-3636538$ & +0.06 & $\ldots$ & -0.04 & +0.31 \\
$18123168-3631555$ & +0.09 & +0.01 & -0.24 & +0.09 \\
$18115398-3623290$ & +0.09 & +0.51 & -0.09 & +0.14 \\
$18123393-3615508$ & +0.11 & $\ldots$ & +0.00 & +0.16 \\
& & & &
\end{tabular}


TABLE 3-Continued

\begin{tabular}{ccccc}
\hline \hline Star ID & {$[\mathrm{Fe} / \mathrm{H}]$} & {$[\mathrm{O} / \mathrm{Fe}]$} & {$[\mathrm{Si} / \mathrm{Fe}]$} & {$[\mathrm{Ca} / \mathrm{Fe}]$} \\
2MASS & & & & \\
\hline $18120689-3643231$ & +0.11 & -0.31 & -0.01 & -0.07 \\
$18130482-3649019$ & +0.13 & +0.11 & -0.13 & $\ldots$ \\
$18110352-3646275$ & +0.14 & +0.01 & -0.07 & -0.05 \\
$18105013-3629557$ & +0.17 & -0.22 & -0.12 & +0.21 \\
$18121897-3630243$ & +0.22 & $\ldots$ & +0.08 & +0.14 \\
$18122029-3652333$ & +0.26 & $\ldots$ & -0.09 & +0.12 \\
\hline & $(1, \mathrm{~b})=(+8.5,+9)$ & & \\
\hline $17311256-1702018$ & -1.26 & +0.76 & +0.31 & +0.34 \\
$17290874-1632431$ & -1.23 & +0.70 & +0.31 & +0.30 \\
$17302885-1639588$ & -1.17 & +0.57 & +0.46 & +0.36 \\
$17304789-1623192$ & -1.14 & +0.64 & +0.36 & +0.44 \\
$17321080-1641479$ & -1.09 & +0.77 & +0.27 & +0.27 \\
$17303589-1638299$ & -0.97 & +0.66 & +0.42 & +0.44 \\
$17290513-1630383$ & -0.96 & +0.84 & +0.57 & +0.40 \\
$17314699-1654460$ & -0.93 & +0.73 & +0.24 & +0.33 \\
$17305076-1615216$ & -0.81 & +0.66 & +0.46 & +0.37 \\
$17323324-1627474$ & -0.80 & +0.38 & +0.54 & $\ldots$ \\
$17312795-1631423$ & -0.78 & +0.56 & +0.33 & +0.15 \\
$17290285-1638344$ & -0.73 & +0.60 & +0.37 & +0.21 \\
$17303378-1703086$ & -0.71 & +0.51 & +0.38 & +0.27 \\
$17304223-1609274$ & -0.64 & +0.59 & +0.39 & +0.15 \\
$17304908-1633101$ & -0.64 & $\ldots$ & +0.31 & +0.14 \\
$17291459-1650085$ & -0.62 & +0.55 & +0.27 & +0.00 \\
$17300674-1638541$ & -0.57 & $\ldots$ & +0.40 & +0.31 \\
$17315010-1653413$ & -0.55 & +0.59 & +0.46 & +0.37 \\
$17295493-1613273$ & -0.54 & $\ldots$ & +0.54 & +0.22 \\
$17311523-1618542$ & -0.52 & $\ldots$ & +0.46 & $\ldots$ \\
$17314811-1621558$ & -0.52 & +0.21 & +0.33 & +0.00 \\
$17304861-1646459$ & -0.51 & +0.56 & +0.31 & +0.11 \\
$17323959-1625298$ & -0.50 & $\ldots$ & +0.43 & +0.22 \\
$17285711-1633566$ & -0.50 & +0.50 & +0.33 & +0.16 \\
$17314673-1641228$ & -0.48 & +0.46 & +0.28 & +0.35 \\
& & & &
\end{tabular}


TABle 3-Continued

\begin{tabular}{ccccc}
\hline \hline Star ID & {$[\mathrm{Fe} / \mathrm{H}]$} & {$[\mathrm{O} / \mathrm{Fe}]$} & {$[\mathrm{Si} / \mathrm{Fe}]$} & {$[\mathrm{Ca} / \mathrm{Fe}]$} \\
2MASS & & & & \\
\hline $17294476-1626118$ & -0.44 & +0.39 & +0.25 & +0.27 \\
$17320650-1643389$ & -0.39 & +0.19 & +0.11 & +0.09 \\
$17314427-1640265$ & -0.39 & +0.46 & +0.30 & +0.19 \\
$17300064-1701075$ & -0.38 & $\ldots$ & +0.08 & -0.01 \\
$17302935-1652277$ & -0.34 & -0.31 & +0.12 & -0.11 \\
$17320652-1619563$ & -0.30 & +0.27 & +0.33 & +0.45 \\
$17304464-1631471$ & -0.29 & +0.69 & +0.25 & +0.24 \\
$17321715-1632467$ & -0.28 & $\ldots$ & +0.30 & -0.04 \\
$17321969-1653156$ & -0.25 & +0.55 & +0.13 & +0.14 \\
$17315690-1630315$ & -0.20 & +0.22 & +0.35 & +0.18 \\
$17312870-1619572$ & -0.19 & +0.30 & +0.14 & +0.03 \\
$17294541-1649018$ & -0.19 & +0.29 & +0.01 & +0.07 \\
$17305067-1644006$ & -0.19 & +0.23 & +0.00 & -0.10 \\
$17312457-1652524$ & -0.18 & +0.48 & +0.29 & +0.20 \\
$17292760-1636235$ & -0.18 & +0.68 & -0.03 & +0.12 \\
$17295978-1633590$ & -0.17 & $\cdots$ & +0.04 & -0.09 \\
$17322323-1649335$ & -0.15 & +0.32 & +0.20 & +0.02 \\
$17321117-1654590$ & -0.13 & +0.08 & +0.12 & +0.25 \\
$17313606-1607539$ & -0.11 & +0.41 & +0.27 & -0.07 \\
$17295331-1653069$ & -0.10 & +0.52 & +0.20 & +0.20 \\
$17302818-1636032$ & -0.07 & +0.32 & +0.05 & +0.10 \\
$17293118-1638081$ & -0.07 & $\cdots$ & -0.01 & +0.00 \\
$17291994-1618302$ & -0.07 & +0.29 & +0.01 & -0.29 \\
$17320457-1646391$ & -0.05 & +0.17 & +0.02 & +0.08 \\
$17310747-1620049$ & -0.04 & +0.44 & +0.09 & +0.26 \\
$17323392-1636330$ & -0.04 & -0.05 & $\ldots$ & +0.11 \\
$17312572-1609092$ & -0.03 & -0.04 & +0.09 & +0.19 \\
$17300015-1609464$ & +0.03 & $\ldots$ & -0.13 & +0.11 \\
$17305894-1637056$ & +0.10 & +0.29 & -0.30 & -0.09 \\
$17291621-1647189$ & +0.12 & +0.18 & -0.12 & -0.27 \\
$17302194-1643531$ & +0.14 & +0.26 & -0.29 & -0.12 \\
$17311959-1643577$ & +0.16 & $\ldots$ & -0.23 & -0.06 \\
& & & &
\end{tabular}


TABle 3-Continued

\begin{tabular}{ccccc}
\hline \hline $\begin{array}{c}\text { Star ID } \\
\text { 2MASS }\end{array}$ & {$[\mathrm{Fe} / \mathrm{H}]$} & {$[\mathrm{O} / \mathrm{Fe}]$} & {$[\mathrm{Si} / \mathrm{Fe}]$} & {$[\mathrm{Ca} / \mathrm{Fe}]$} \\
\hline $17311229-1659047$ & +0.20 & $\ldots$ & -0.30 & -0.13 \\
$17313313-1659216$ & +0.21 & +0.17 & -0.18 & -0.04 \\
$17301749-1629529$ & +0.23 & -0.08 & -0.26 & -0.07 \\
$17294261-1622440$ & +0.23 & $\ldots$ & -0.38 & -0.23 \\
$17323625-1637551$ & +0.26 & -0.33 & -0.28 & +0.07 \\
$17314292-1620036$ & +0.28 & $\ldots$ & -0.32 & -0.22 \\
$17310955-1635152$ & +0.32 & -0.27 & -0.12 & -0.11 \\
$17304312-1630318$ & +0.37 & $\ldots$ & -0.32 & +0.09 \\
$17285469-1627232$ & +0.42 & +0.05 & -0.30 & -0.03 \\
$17310360-1700146$ & +0.43 & $\ldots$ & -0.18 & +0.07 \\
$17295898-1652101$ & +0.59 & -0.17 & -0.44 & -0.11 \\
\hline
\end{tabular}


TABLE 4

LINE LIST

\begin{tabular}{|c|c|c|c|c|}
\hline Element & $\begin{array}{c}\text { Wavelength } \\
(\AA)\end{array}$ & $\begin{array}{l}\text { E.P. } \\
(\mathrm{eV})\end{array}$ & $\log g f$ & Arcturus $[\mathrm{Fe} / \mathrm{H}]$ or $[\mathrm{X} / \mathrm{Fe}]$ \\
\hline Fe I & 6056.00 & 4.73 & -0.40 & -0.50 \\
\hline $\mathrm{Fe} \mathrm{I}$ & 6062.85 & 2.18 & -4.00 & -0.50 \\
\hline Fe I & 6065.48 & 2.61 & -1.56 & -0.50 \\
\hline Fe I & 6078.49 & 4.80 & -0.46 & -0.50 \\
\hline $\mathrm{Fe} \mathrm{I}$ & 6079.01 & 4.65 & -1.13 & -0.50 \\
\hline Fe I & 6093.64 & 4.61 & -1.47 & -0.50 \\
\hline Fe I & 6094.37 & 4.65 & -1.70 & -0.50 \\
\hline Fe I & 6096.66 & 3.98 & -1.96 & -0.50 \\
\hline Fe I & 6098.24 & 4.56 & -1.80 & -0.50 \\
\hline $\mathrm{Fe} \mathrm{I}$ & 6102.17 & 4.83 & -0.30 & -0.50 \\
\hline $\mathrm{Fe} \mathrm{I}$ & 6105.13 & 4.55 & -1.97 & -0.50 \\
\hline Fe I & 6120.25 & 0.92 & -5.94 & -0.50 \\
\hline Fe I & 6127.91 & 4.14 & -1.49 & -0.50 \\
\hline Fe I & 6151.62 & 2.18 & -3.27 & -0.50 \\
\hline Fe I & 6156.81 & 4.96 & -2.24 & -0.50 \\
\hline Fe I & 6165.36 & 4.14 & -1.64 & -0.50 \\
\hline Fe I & 6173.34 & 2.22 & -2.87 & -0.50 \\
\hline Fe I & 6187.40 & 2.83 & -4.14 & -0.50 \\
\hline Fe I & 6187.99 & 3.94 & -1.62 & -0.50 \\
\hline Fe I & 6200.31 & 2.61 & -2.33 & -0.50 \\
\hline Fe I & 6207.23 & 4.99 & -1.80 & -0.50 \\
\hline Fe I & 6226.74 & 3.88 & -2.10 & -0.50 \\
\hline Fe I & 6232.64 & 3.65 & -1.11 & -0.50 \\
\hline Fe I & 6240.65 & 2.22 & -3.34 & -0.50 \\
\hline Fe I & 6246.32 & 3.60 & -0.77 & -0.50 \\
\hline Fe I & 6252.56 & 2.40 & -1.63 & -0.50 \\
\hline Fe I & 6270.23 & 2.86 & -2.57 & -0.50 \\
\hline Fe I & 6290.54 & 2.59 & -4.39 & -0.50 \\
\hline Fe I & 6297.79 & 2.22 & -2.67 & -0.50 \\
\hline Fe I & 6301.50 & 3.65 & -0.67 & -0.50 \\
\hline Fe I & 6302.49 & 3.69 & -1.12 & -0.50 \\
\hline Fe I & 6307.85 & 3.64 & -3.27 & -0.50 \\
\hline
\end{tabular}


TABle 4-Continued

\begin{tabular}{|c|c|c|c|c|}
\hline Element & $\begin{array}{l}\text { Wavelength } \\
\qquad(\AA)\end{array}$ & $\begin{array}{l}\text { E.P. } \\
(\mathrm{eV})\end{array}$ & $\log g f$ & Arcturus $[\mathrm{Fe} / \mathrm{H}]$ or $[\mathrm{X} / \mathrm{Fe}]$ \\
\hline $\mathrm{Fe} I$ & 6315.81 & 4.08 & -1.57 & -0.50 \\
\hline $\mathrm{Fe} I$ & 6322.69 & 2.59 & -2.26 & -0.50 \\
\hline Fe I & 6330.85 & 4.73 & -1.16 & -0.50 \\
\hline Fe I & 6574.25 & 0.99 & -4.76 & -0.50 \\
\hline Fe I & 6591.33 & 4.59 & -1.96 & -0.50 \\
\hline $\mathrm{Fe} I$ & 6593.88 & 2.43 & -2.15 & -0.50 \\
\hline Fe I & 6597.56 & 4.80 & -1.03 & -0.50 \\
\hline $\mathrm{Fe} I$ & 6627.56 & 4.55 & -1.40 & -0.50 \\
\hline $\mathrm{Fe} I$ & 6633.75 & 4.56 & -0.89 & -0.50 \\
\hline Fe I & 6646.97 & 2.61 & -3.80 & -0.50 \\
\hline Fe I & 6648.12 & 1.01 & -5.67 & -0.50 \\
\hline $\mathrm{Fe} I$ & 6653.91 & 4.15 & -2.25 & -0.50 \\
\hline Fe I & 6667.42 & 2.45 & -4.43 & -0.50 \\
\hline Fe I & 6678.00 & 2.69 & -1.17 & -0.50 \\
\hline Fe I & 6692.27 & 4.08 & -3.15 & -0.50 \\
\hline Fe I & 6703.58 & 2.76 & -2.88 & -0.50 \\
\hline Fe I & 6704.48 & 4.22 & -2.60 & -0.50 \\
\hline Fe I & 6710.32 & 1.48 & -4.80 & -0.50 \\
\hline $\mathrm{Fe} I$ & 6713.74 & 4.80 & -1.53 & -0.50 \\
\hline Fe I & 6725.36 & 4.10 & -2.20 & -0.50 \\
\hline $\mathrm{Fe} I$ & 6726.67 & 4.61 & -1.07 & -0.50 \\
\hline $\mathrm{Fe} I$ & 6730.29 & 4.91 & -2.24 & -0.50 \\
\hline Fe I & 6733.15 & 4.64 & -1.58 & -0.50 \\
\hline Fe I & 6739.52 & 1.56 & -5.03 & -0.50 \\
\hline Fe I & 6745.96 & 4.08 & -2.85 & -0.50 \\
\hline Fe I & 6746.95 & 2.61 & -4.43 & -0.50 \\
\hline Fe I & 6750.16 & 2.42 & -2.57 & -0.50 \\
\hline Fe I & 6793.27 & 4.07 & -2.33 & -0.50 \\
\hline Fe I & 6810.26 & 4.61 & -1.10 & -0.50 \\
\hline O I & 6300.31 & 0.00 & -10.00 & +0.48 \\
\hline Si I & 6145.01 & 5.62 & -1.50 & +0.35 \\
\hline Si I & 6155.13 & 5.62 & -0.97 & +0.35 \\
\hline
\end{tabular}


TABLE 4-Continued

\begin{tabular}{ccccc}
\hline \hline Element & $\begin{array}{c}\text { Wavelength } \\
(\AA)\end{array}$ & $\begin{array}{c}\text { E.P. } \\
(\mathrm{eV})\end{array}$ & log gf & Arcturus $[\mathrm{Fe} / \mathrm{H}]$ or $[\mathrm{X} / \mathrm{Fe}]$ \\
\hline Ca I & 6156.01 & 2.52 & -2.61 & +0.21 \\
Ca I & 6166.44 & 2.52 & -1.24 & +0.21 \\
Ca I & 6169.04 & 2.52 & -0.90 & +0.21 \\
Ca I & 6169.56 & 2.52 & -0.67 & +0.21 \\
\hline
\end{tabular}


TABLE 5A

Abundance UnCertainty: Iron

\begin{tabular}{ccccccc}
\hline \hline Star ID & $\Delta \mathrm{T}_{\text {eff }}$ & $\Delta \log (\mathrm{g})$ & $\Delta[\mathrm{M} / \mathrm{H}]$ & $\Delta \mathrm{V}_{\mathrm{t}}$ & $\sigma / \sqrt{ }(\mathrm{N})$ & $\Delta$ Total \\
2MASS & $+100 \mathrm{~K}$ & $+0.3 \mathrm{cgs}$ & $+0.3 \mathrm{dex}$ & $+0.3 \mathrm{~km} \mathrm{~s}$ & & \\
\hline \multicolumn{7}{c}{$(\mathrm{l}, \mathrm{b})=(-5.5,-7)$} \\
\hline $18011989-3723462$ & +0.10 & +0.02 & +0.01 & -0.06 & 0.03 & 0.12 \\
$18003452-3729217$ & +0.10 & +0.01 & +0.00 & -0.04 & 0.03 & 0.11 \\
$18011692-3722508$ & +0.10 & +0.03 & +0.02 & -0.06 & 0.04 & 0.13 \\
$18005584-3739103$ & +0.08 & +0.03 & +0.03 & -0.06 & 0.03 & 0.11 \\
$18015010-3735279$ & +0.08 & +0.04 & +0.03 & -0.07 & 0.03 & 0.12 \\
$18005916-3727585$ & +0.08 & +0.03 & +0.03 & -0.07 & 0.03 & 0.12 \\
$18005027-3707505$ & +0.08 & +0.03 & +0.02 & -0.06 & 0.04 & 0.11 \\
$18005234-3715478$ & +0.07 & +0.05 & +0.04 & -0.09 & 0.03 & 0.13 \\
$18020106-3737561$ & +0.07 & +0.05 & +0.04 & -0.08 & 0.04 & 0.13 \\
$17595541-3713447$ & +0.07 & +0.03 & +0.03 & -0.08 & 0.04 & 0.12 \\
$17595664-3725304$ & +0.07 & +0.03 & +0.03 & -0.09 & 0.03 & 0.13 \\
$18021345-3730371$ & +0.03 & +0.07 & +0.05 & -0.09 & 0.04 & 0.13 \\
$17594241-3718247$ & +0.05 & +0.05 & +0.04 & -0.08 & 0.04 & 0.12 \\
$18013342-3726339$ & +0.06 & +0.04 & +0.04 & -0.08 & 0.03 & 0.12 \\
$18003204-3726008$ & +0.02 & +0.06 & +0.06 & -0.11 & 0.03 & 0.14 \\
$18013580-3740055$ & +0.02 & +0.07 & +0.06 & -0.10 & 0.04 & 0.14 \\
$18013799-3725131$ & +0.02 & +0.07 & +0.06 & -0.10 & 0.03 & 0.14 \\
$18022149-3713494$ & +0.03 & +0.07 & +0.06 & -0.10 & 0.03 & 0.14 \\
$18011408-3711299$ & +0.06 & +0.04 & +0.04 & -0.09 & 0.03 & 0.13 \\
$18021275-3737543$ & +0.06 & +0.05 & +0.04 & -0.10 & 0.04 & 0.14 \\
$17595027-3727293$ & +0.02 & +0.06 & +0.06 & -0.10 & 0.05 & 0.14 \\
$18002115-3715519$ & +0.01 & +0.07 & +0.08 & -0.11 & 0.03 & 0.16 \\
$17595366-3728186$ & +0.06 & +0.05 & +0.05 & -0.09 & 0.04 & 0.14
\end{tabular}


TABLE 5A-Continued

\begin{tabular}{ccccccc}
\hline \hline Star ID & $\Delta \mathrm{T}_{\text {eff }}$ & $\Delta \log (\mathrm{g})$ & $\Delta[\mathrm{M} / \mathrm{H}]$ & $\Delta \mathrm{V}_{\mathrm{t}}$ & $\sigma / \sqrt{ }(\mathrm{N})$ & $\Delta$ Total \\
2MASS & $+100 \mathrm{~K}$ & $+0.3 \mathrm{cgs}$ & $+0.3 \mathrm{dex}$ & $+0.3 \mathrm{~km} \mathrm{~s}$ & & \\
\hline $18001354-3709122$ & +0.01 & +0.09 & +0.08 & -0.11 & 0.03 & 0.17 \\
$18013960-3723398$ & +0.01 & +0.09 & +0.08 & -0.12 & 0.03 & 0.17 \\
$18021862-3734204$ & +0.01 & +0.08 & +0.08 & -0.11 & 0.05 & 0.17 \\
$18000535-3735315$ & +0.01 & +0.08 & +0.08 & -0.12 & 0.03 & 0.17 \\
$18013848-3721442$ & +0.02 & +0.08 & +0.08 & -0.11 & 0.04 & 0.16 \\
$17594792-3721422$ & +0.04 & +0.04 & +0.06 & -0.11 & 0.03 & 0.14 \\
$18011941-3721250$ & +0.01 & +0.09 & +0.08 & -0.10 & 0.04 & 0.16 \\
$18004824-3718074$ & +0.00 & +0.08 & +0.09 & -0.12 & 0.04 & 0.17 \\
$18014343-3708232$ & +0.01 & +0.09 & +0.08 & -0.12 & 0.03 & 0.17 \\
$18005852-3711524$ & +0.06 & +0.05 & +0.04 & -0.10 & 0.03 & 0.14 \\
$18020816-3714015$ & +0.05 & +0.05 & +0.05 & -0.10 & 0.04 & 0.14 \\
$18010932-3702079$ & +0.00 & +0.09 & +0.09 & -0.11 & 0.04 & 0.17 \\
$18010065-3740456$ & +0.00 & +0.09 & +0.09 & -0.12 & 0.04 & 0.18 \\
$17595640-3710383$ & +0.03 & +0.04 & +0.06 & -0.12 & 0.02 & 0.14 \\
$18012462-3738382$ & +0.05 & +0.06 & +0.05 & -0.10 & 0.04 & 0.14 \\
$18002880-3710171$ & +0.06 & +0.05 & +0.04 & -0.09 & 0.03 & 0.13 \\
$18024484-3714159$ & +0.06 & +0.05 & +0.04 & -0.11 & 0.03 & 0.14 \\
$18013206-3708133$ & +0.06 & +0.05 & +0.04 & -0.11 & 0.05 & 0.15 \\
$18004074-3740058$ & +0.03 & +0.05 & +0.05 & -0.11 & 0.03 & 0.14 \\
$18000013-3715053$ & +0.02 & +0.06 & +0.06 & -0.12 & 0.03 & 0.15 \\
$18015431-3717070$ & +0.04 & +0.06 & +0.05 & -0.12 & 0.05 & 0.16 \\
$18021006-3738193$ & +0.06 & +0.05 & +0.04 & -0.10 & 0.03 & 0.14 \\
$17595442-3721118$ & +0.01 & +0.07 & +0.05 & -0.12 & 0.03 & 0.15 \\
$18021721-3736096$ & +0.05 & +0.06 & +0.04 & -0.10 & 0.05 & 0.14
\end{tabular}


TABLE 5A-Continued

\begin{tabular}{ccccccc}
\hline \hline Star ID & $\Delta \mathrm{T}_{\text {eff }}$ & $\Delta \log (\mathrm{g})$ & $\Delta[\mathrm{M} / \mathrm{H}]$ & $\Delta \mathrm{V}_{\mathrm{t}}$ & $\sigma / \sqrt{ }(\mathrm{N})$ & $\Delta$ Total \\
2MASS & $+100 \mathrm{~K}$ & $+0.3 \mathrm{cgs}$ & $+0.3 \mathrm{dex}$ & $+0.3 \mathrm{~km} \mathrm{~s}$ & & \\
\hline $18005096-3733499$ & +0.01 & +0.05 & +0.06 & -0.13 & 0.03 & 0.15 \\
$18005923-3707111$ & -0.01 & +0.06 & +0.06 & -0.13 & 0.04 & 0.16 \\
$18011851-3706092$ & +0.00 & +0.07 & +0.06 & -0.12 & 0.03 & 0.15 \\
$18013395-3705551$ & -0.01 & +0.08 & +0.06 & -0.13 & 0.03 & 0.17 \\
$18022648-3728188$ & +0.04 & +0.07 & +0.05 & -0.11 & 0.02 & 0.15 \\
$18024919-3727234$ & +0.05 & +0.07 & +0.05 & -0.11 & 0.05 & 0.16 \\
$18023660-3716289$ & +0.04 & +0.06 & +0.05 & -0.11 & 0.03 & 0.14 \\
$17595509-3711099$ & +0.02 & +0.06 & +0.06 & -0.12 & 0.03 & 0.15 \\
$18005395-3731231$ & -0.01 & +0.08 & +0.06 & -0.12 & 0.04 & 0.16 \\
$18010322-3705447$ & +0.05 & +0.06 & +0.05 & -0.11 & 0.03 & 0.15 \\
$18014769-3739220$ & +0.05 & +0.06 & +0.05 & -0.13 & 0.04 & 0.16 \\
$17594807-3715283$ & +0.04 & +0.07 & +0.06 & -0.13 & 0.04 & 0.17 \\
$18013618-3706311$ & +0.00 & +0.05 & +0.07 & -0.14 & 0.04 & 0.17 \\
$18004314-3730156$ & +0.03 & +0.07 & +0.07 & -0.13 & 0.05 & 0.17 \\
$18014303-3740054$ & +0.01 & +0.06 & +0.07 & -0.13 & 0.03 & 0.16 \\
$18003751-3728200$ & -0.02 & +0.09 & +0.08 & -0.15 & 0.03 & 0.20 \\
$18011170-3727118$ & +0.06 & +0.05 & +0.05 & -0.12 & 0.04 & 0.16 \\
$18023846-3718345$ & +0.01 & +0.07 & +0.08 & -0.12 & 0.04 & 0.17 \\
$18011671-3722213$ & +0.05 & +0.05 & +0.06 & -0.13 & 0.04 & 0.16 \\
$18005985-3734507$ & -0.01 & +0.06 & +0.09 & -0.14 & 0.03 & 0.18 \\
$18000430-3711051$ & +0.00 & +0.06 & +0.08 & -0.14 & 0.02 & 0.17 \\
$18003552-3729476$ & +0.01 & +0.07 & +0.08 & -0.13 & 0.04 & 0.17 \\
$18001817-3732243$ & +0.03 & +0.07 & +0.07 & -0.14 & 0.03 & 0.18 \\
$18013039-3713086$ & -0.01 & +0.08 & +0.08 & -0.13 & 0.04 & 0.18
\end{tabular}


TABLE 5A-Continued

\begin{tabular}{ccccccc}
\hline \hline Star ID & $\begin{array}{c}\Delta \mathrm{T}_{\text {eff }} \\
\text { 2MASS }\end{array}$ & $\begin{array}{c}\Delta \log (\mathrm{g}) \\
+0.3 \mathrm{cgs}\end{array}$ & $\begin{array}{c}\Delta[\mathrm{M} / \mathrm{H}] \\
+0.3 \mathrm{dex}\end{array}$ & $\begin{array}{c}\Delta \mathrm{V}_{\mathrm{t}} \\
+0.3 \mathrm{~km} \mathrm{~s}\end{array}$ & $\sigma / \sqrt{ }(\mathrm{N})$ & $\Delta$ Total \\
\hline $18012564-3741052$ & +0.01 & +0.08 & +0.08 & -0.13 & 0.05 & 0.18 \\
$18025713-3718318$ & -0.01 & +0.08 & +0.08 & -0.14 & 0.05 & 0.19 \\
$18011599-3741385$ & +0.00 & +0.07 & +0.08 & -0.14 & 0.04 & 0.18 \\
$18012944-3719488$ & +0.05 & +0.06 & +0.06 & -0.12 & 0.03 & 0.16 \\
$18020361-3719086$ & +0.02 & +0.08 & +0.07 & -0.13 & 0.04 & 0.17 \\
$18024024-3728187$ & -0.02 & +0.07 & +0.08 & -0.14 & 0.06 & 0.19 \\
$18000412-3717266$ & -0.02 & +0.06 & +0.08 & -0.14 & 0.04 & 0.18 \\
$18011177-3703344$ & +0.00 & +0.07 & +0.08 & -0.15 & 0.03 & 0.19 \\
$18001410-3725181$ & -0.03 & +0.07 & +0.08 & -0.15 & 0.03 & 0.19 \\
$18020300-3719418$ & -0.02 & +0.06 & +0.07 & -0.16 & 0.04 & 0.19 \\
$18004683-3703252$ & -0.03 & +0.08 & +0.07 & -0.15 & 0.06 & 0.20 \\
$18015404-3722564$ & +0.00 & +0.07 & +0.06 & -0.15 & 0.04 & 0.18 \\
$18013974-3704068$ & +0.00 & +0.07 & +0.08 & -0.15 & 0.03 & 0.19 \\
$18020704-3708064$ & +0.03 & +0.08 & +0.06 & -0.15 & 0.04 & 0.19 \\
$18023393-3730273$ & +0.03 & +0.08 & +0.06 & -0.14 & 0.06 & 0.18 \\
$18001720-3708374$ & -0.03 & +0.06 & +0.09 & -0.16 & 0.03 & 0.20 \\
$18003663-3708547$ & -0.01 & +0.08 & +0.08 & -0.16 & 0.04 & 0.20 \\
$18002569-3729578$ & +0.02 & +0.09 & +0.07 & -0.16 & 0.03 & 0.20 \\
$17594881-3716203$ & -0.01 & +0.09 & +0.08 & -0.17 & 0.04 & 0.21 \\
$18011201-3740090$ & +0.02 & +0.08 & +0.07 & -0.17 & 0.05 & 0.21 \\
\hline & & & $(1, \mathrm{~b})=(-4,-9)$ & & & \\
\hline $18122552-3653049$ & +0.10 & +0.01 & +0.00 & -0.06 & 0.05 & 0.13 \\
$18120653-3654264$ & +0.10 & +0.01 & +0.00 & -0.06 & 0.02 & 0.12 \\
$18110179-3630394$ & +0.10 & +0.01 & +0.01 & -0.05 & 0.04 & 0.12
\end{tabular}


TABLE 5A-Continued

\begin{tabular}{ccccccc}
\hline \hline Star ID & $\Delta \mathrm{T}_{\text {eff }}$ & $\Delta \log (\mathrm{g})$ & $\Delta[\mathrm{M} / \mathrm{H}]$ & $\Delta \mathrm{V}_{\mathrm{t}}$ & $\sigma / \sqrt{ }(\mathrm{N})$ & $\Delta$ Total \\
2MASS & $+100 \mathrm{~K}$ & $+0.3 \mathrm{cgs}$ & $+0.3 \mathrm{dex}$ & $+0.3 \mathrm{~km} \mathrm{~s}$ & & \\
\hline $18124892-3626403$ & +0.09 & +0.01 & +0.01 & -0.05 & 0.04 & 0.11 \\
$18135164-3643463$ & +0.10 & +0.02 & +0.02 & -0.05 & 0.05 & 0.13 \\
$18124339-3635339$ & +0.10 & +0.02 & +0.02 & -0.06 & 0.03 & 0.12 \\
$18113921-3652069$ & +0.08 & +0.04 & +0.03 & -0.06 & 0.04 & 0.12 \\
$18111325-3646007$ & +0.09 & +0.02 & +0.03 & -0.07 & 0.04 & 0.13 \\
$18113833-3626322$ & +0.06 & +0.05 & +0.05 & -0.07 & 0.03 & 0.12 \\
$18111018-3639350$ & +0.07 & +0.04 & +0.04 & -0.08 & 0.03 & 0.12 \\
$18135243-3630481$ & +0.09 & +0.02 & +0.02 & -0.06 & 0.03 & 0.12 \\
$18121150-3627559$ & +0.08 & +0.04 & +0.04 & -0.08 & 0.03 & 0.13 \\
$18111963-3647245$ & +0.07 & +0.05 & +0.04 & -0.08 & 0.04 & 0.13 \\
$18140023-3635461$ & +0.06 & +0.05 & +0.04 & -0.08 & 0.03 & 0.12 \\
$18135881-3639345$ & +0.06 & +0.05 & +0.04 & -0.08 & 0.04 & 0.13 \\
$18121436-3654551$ & +0.05 & +0.07 & +0.05 & -0.09 & 0.03 & 0.14 \\
$18124228-3655079$ & +0.04 & +0.06 & +0.06 & -0.08 & 0.02 & 0.12 \\
$18122530-3637366$ & +0.04 & +0.07 & +0.05 & -0.09 & 0.03 & 0.13 \\
$18131370-3651584$ & +0.06 & +0.05 & +0.04 & -0.10 & 0.04 & 0.14 \\
$18134668-3623541$ & +0.05 & +0.05 & +0.05 & -0.08 & 0.03 & 0.12 \\
$18105081-3642137$ & +0.07 & +0.03 & +0.03 & -0.09 & 0.03 & 0.13 \\
$18124219-3631172$ & +0.05 & +0.06 & +0.05 & -0.09 & 0.04 & 0.14 \\
$18133688-3647054$ & +0.07 & +0.03 & +0.03 & -0.08 & 0.03 & 0.12 \\
$18123142-3639294$ & +0.02 & +0.08 & +0.05 & -0.09 & 0.04 & 0.14 \\
$18133038-3632229$ & +0.07 & +0.03 & +0.02 & -0.07 & 0.03 & 0.11 \\
$18133292-3624179$ & +0.08 & +0.03 & +0.02 & -0.07 & 0.04 & 0.12 \\
$18111681-3636318$ & +0.02 & +0.06 & +0.06 & -0.11 & 0.02 & 0.14
\end{tabular}


TABLE 5A-Continued

\begin{tabular}{ccccccc}
\hline \hline Star ID & $\Delta \mathrm{T}_{\text {eff }}$ & $\Delta \log (\mathrm{g})$ & $\Delta[\mathrm{M} / \mathrm{H}]$ & $\Delta \mathrm{V}_{\mathrm{t}}$ & $\sigma / \sqrt{ }(\mathrm{N})$ & $\Delta$ Total \\
2MASS & $+100 \mathrm{~K}$ & $+0.3 \mathrm{cgs}$ & $+0.3 \mathrm{dex}$ & $+0.3 \mathrm{~km} \mathrm{~s}$ & & \\
\hline $18105004-3640063$ & +0.07 & +0.04 & +0.04 & -0.08 & 0.04 & 0.13 \\
$18112334-3634194$ & +0.06 & +0.04 & +0.04 & -0.09 & 0.03 & 0.13 \\
$18131027-3632343$ & +0.04 & +0.06 & +0.05 & -0.10 & 0.03 & 0.14 \\
$18110293-3628041$ & +0.03 & +0.07 & +0.06 & -0.09 & 0.03 & 0.14 \\
$18132040-3623075$ & +0.05 & +0.04 & +0.05 & -0.09 & 0.03 & 0.12 \\
$18135588-3629188$ & +0.06 & +0.05 & +0.05 & -0.08 & 0.02 & 0.12 \\
$18105075-3635061$ & +0.02 & +0.07 & +0.06 & -0.11 & 0.04 & 0.15 \\
$18104188-3634541$ & +0.07 & +0.04 & +0.03 & -0.08 & 0.04 & 0.12 \\
$18132998-3628578$ & +0.02 & +0.08 & +0.07 & -0.10 & 0.03 & 0.15 \\
$18130544-3616563$ & +0.02 & +0.07 & +0.06 & -0.10 & 0.03 & 0.14 \\
$18115908-3654510$ & +0.02 & +0.08 & +0.07 & -0.12 & 0.03 & 0.16 \\
$18121077-3639094$ & +0.03 & +0.06 & +0.06 & -0.10 & 0.03 & 0.14 \\
$18120614-3629571$ & +0.05 & +0.04 & +0.06 & -0.11 & 0.03 & 0.14 \\
$18120213-3641599$ & +0.06 & +0.04 & +0.04 & -0.10 & 0.03 & 0.13 \\
$18123350-3654398$ & +0.01 & +0.08 & +0.08 & -0.11 & 0.05 & 0.17 \\
$18120973-3654184$ & +0.00 & +0.09 & +0.09 & -0.10 & 0.04 & 0.17 \\
$18105641-3632359$ & +0.04 & +0.04 & +0.06 & -0.11 & 0.03 & 0.14 \\
$18125106-3654091$ & +0.06 & +0.04 & +0.04 & -0.11 & 0.04 & 0.14 \\
$18111581-3648469$ & +0.00 & +0.08 & +0.09 & -0.12 & 0.03 & 0.17 \\
$18131387-3630130$ & +0.02 & +0.08 & +0.08 & -0.11 & 0.05 & 0.17 \\
$18123592-3634476$ & +0.00 & +0.09 & +0.09 & -0.10 & 0.03 & 0.16 \\
$18135736-3638266$ & +0.02 & +0.07 & +0.08 & -0.12 & 0.03 & 0.16 \\
$18133233-3626133$ & +0.00 & +0.09 & +0.09 & -0.11 & 0.04 & 0.17 \\
$18125003-3630269$ & +0.04 & +0.05 & +0.06 & -0.10 & 0.03 & 0.14
\end{tabular}


TABLE 5A-Continued

\begin{tabular}{ccccccc}
\hline \hline Star ID & $\Delta \mathrm{T}_{\text {eff }}$ & $\Delta \log (\mathrm{g})$ & $\Delta[\mathrm{M} / \mathrm{H}]$ & $\Delta \mathrm{V}_{\mathrm{t}}$ & $\sigma / \sqrt{ }(\mathrm{N})$ & $\Delta$ Total \\
2MASS & $+100 \mathrm{~K}$ & $+0.3 \mathrm{cgs}$ & $+0.3 \mathrm{dex}$ & $+0.3 \mathrm{~km} \mathrm{~s}$ & & \\
\hline $18130723-3618385$ & +0.05 & +0.05 & +0.05 & -0.11 & 0.03 & 0.14 \\
$18125800-3616427$ & +0.07 & +0.04 & +0.03 & -0.10 & 0.03 & 0.14 \\
$18111323-3637030$ & +0.05 & +0.05 & +0.05 & -0.12 & 0.03 & 0.15 \\
$18130427-3630012$ & +0.06 & +0.05 & +0.04 & -0.11 & 0.03 & 0.14 \\
$18124614-3617326$ & +0.02 & +0.07 & +0.07 & -0.11 & 0.03 & 0.15 \\
$18120399-3615387$ & +0.01 & +0.07 & +0.07 & -0.13 & 0.03 & 0.17 \\
$18123475-3624478$ & +0.04 & +0.06 & +0.05 & -0.10 & 0.05 & 0.14 \\
$18115661-3617533$ & +0.06 & +0.05 & +0.04 & -0.10 & 0.04 & 0.14 \\
$18113299-3623341$ & +0.01 & +0.06 & +0.07 & -0.12 & 0.02 & 0.15 \\
$18111553-3628156$ & +0.01 & +0.08 & +0.07 & -0.12 & 0.03 & 0.16 \\
$18125907-3644275$ & +0.06 & +0.05 & +0.04 & -0.09 & 0.04 & 0.13 \\
$18111726-3620302$ & +0.03 & +0.06 & +0.05 & -0.10 & 0.04 & 0.14 \\
$18111579-3625114$ & -0.01 & +0.09 & +0.06 & -0.13 & 0.03 & 0.17 \\
$18131305-3650449$ & +0.04 & +0.06 & +0.05 & -0.12 & 0.03 & 0.15 \\
$18123152-3636498$ & -0.01 & +0.08 & +0.06 & -0.13 & 0.03 & 0.17 \\
$18125914-3619208$ & +0.04 & +0.06 & +0.04 & -0.12 & 0.02 & 0.15 \\
$18115073-3650493$ & +0.03 & +0.06 & +0.05 & -0.10 & 0.04 & 0.14 \\
$18114648-3625169$ & +0.06 & +0.05 & +0.04 & -0.11 & 0.04 & 0.15 \\
$18113751-3629139$ & -0.01 & +0.08 & +0.06 & -0.13 & 0.03 & 0.17 \\
$18122243-3637539$ & +0.02 & +0.06 & +0.05 & -0.12 & 0.03 & 0.15 \\
$18133366-3644575$ & +0.06 & +0.05 & +0.04 & -0.11 & 0.03 & 0.14 \\
$18134702-3641477$ & +0.06 & +0.05 & +0.04 & -0.11 & 0.03 & 0.14 \\
$18125228-3618294$ & +0.05 & +0.06 & +0.04 & -0.12 & 0.04 & 0.15 \\
$18110942-3647198$ & +0.06 & +0.06 & +0.04 & -0.11 & 0.04 & 0.15
\end{tabular}


TABLE 5A-Continued

\begin{tabular}{ccccccc}
\hline \hline Star ID & $\Delta \mathrm{T}_{\text {eff }}$ & $\Delta \log (\mathrm{g})$ & $\Delta[\mathrm{M} / \mathrm{H}]$ & $\Delta \mathrm{V}_{\mathrm{t}}$ & $\sigma / \sqrt{ }(\mathrm{N})$ & $\Delta$ Total \\
2MASS & $+100 \mathrm{~K}$ & $+0.3 \mathrm{cgs}$ & $+0.3 \mathrm{dex}$ & $+0.3 \mathrm{~km} \mathrm{~s}$ & & \\
\hline $18104824-3638507$ & -0.01 & +0.08 & +0.06 & -0.14 & 0.03 & 0.17 \\
$18122301-3629462$ & -0.02 & +0.08 & +0.06 & -0.13 & 0.03 & 0.17 \\
$18133332-3645578$ & -0.02 & +0.08 & +0.06 & -0.12 & 0.04 & 0.16 \\
$18123434-3652241$ & +0.02 & +0.06 & +0.06 & -0.12 & 0.03 & 0.15 \\
$18112311-3625456$ & +0.04 & +0.07 & +0.05 & -0.11 & 0.03 & 0.15 \\
$18125463-3649277$ & +0.02 & +0.06 & +0.06 & -0.12 & 0.03 & 0.15 \\
$18123925-3620465$ & -0.01 & +0.07 & +0.06 & -0.14 & 0.03 & 0.17 \\
$18110629-3632146$ & +0.01 & +0.05 & +0.06 & -0.13 & 0.03 & 0.15 \\
$18133687-3639590$ & +0.05 & +0.06 & +0.05 & -0.11 & 0.04 & 0.15 \\
$18111505-3620576$ & +0.05 & +0.06 & +0.05 & -0.13 & 0.03 & 0.16 \\
$18120832-3628243$ & +0.01 & +0.06 & +0.06 & -0.13 & 0.03 & 0.16 \\
$18130815-3631460$ & -0.02 & +0.09 & +0.07 & -0.15 & 0.04 & 0.19 \\
$18120407-3636481$ & -0.01 & +0.07 & +0.07 & -0.14 & 0.03 & 0.17 \\
$18135786-3640300$ & +0.01 & +0.08 & +0.07 & -0.15 & 0.04 & 0.19 \\
$18131228-3619138$ & +0.02 & +0.06 & +0.07 & -0.13 & 0.03 & 0.16 \\
$18113372-3650200$ & +0.00 & +0.07 & +0.08 & -0.14 & 0.03 & 0.18 \\
$18115904-3650428$ & -0.01 & +0.07 & +0.08 & -0.15 & 0.03 & 0.19 \\
$18115448-3641161$ & +0.02 & +0.07 & +0.07 & -0.13 & 0.04 & 0.17 \\
$18123678-3649019$ & +0.03 & +0.06 & +0.07 & -0.14 & 0.03 & 0.17 \\
$18113965-3645408$ & +0.00 & +0.07 & +0.08 & -0.15 & 0.04 & 0.19 \\
$18135362-3636538$ & +0.03 & +0.09 & +0.07 & -0.13 & 0.03 & 0.18 \\
$18123168-3631555$ & +0.05 & +0.06 & +0.06 & -0.13 & 0.04 & 0.17 \\
$18115398-3623290$ & -0.02 & +0.07 & +0.09 & -0.13 & 0.03 & 0.18 \\
$18123393-3615508$ & +0.04 & +0.06 & +0.06 & -0.13 & 0.03 & 0.16
\end{tabular}


TABLE 5A-Continued

\begin{tabular}{ccccccc}
\hline \hline Star ID & $\Delta \mathrm{T}_{\text {eff }}$ & $\begin{array}{c}\Delta \log (\mathrm{g}) \\
\text { 2MASS }\end{array}$ & $\begin{array}{c}\Delta[\mathrm{M} / \mathrm{H}] \\
+100 \mathrm{~K}\end{array}$ & $\begin{array}{c}\Delta \mathrm{V}_{\mathrm{t}} \\
+3 \mathrm{cgs}\end{array}$ & $\sigma / \sqrt{ }(\mathrm{N})$ & $\Delta$ Total \\
$+0.3 \mathrm{~km} \mathrm{~s}$ & & \\
\hline $18120689-3643231$ & +0.03 & +0.08 & +0.07 & -0.13 & 0.03 & 0.17 \\
$18130482-3649019$ & -0.02 & +0.06 & +0.08 & -0.13 & 0.05 & 0.17 \\
$18110352-3646275$ & -0.02 & +0.08 & +0.08 & -0.15 & 0.03 & 0.19 \\
$18105013-3629557$ & +0.01 & +0.07 & +0.08 & -0.15 & 0.02 & 0.19 \\
$18121897-3630243$ & +0.04 & +0.06 & +0.05 & -0.14 & 0.03 & 0.17 \\
$18122029-3652333$ & +0.02 & +0.08 & +0.06 & -0.15 & 0.04 & 0.19 \\
\hline & & $(1, \mathrm{~b})=(+8.5,+9)$ & & & \\
\hline $17311256-1702018$ & +0.07 & +0.05 & +0.04 & -0.08 & 0.04 & 0.13 \\
$17290874-1632431$ & +0.05 & +0.06 & +0.05 & -0.08 & 0.03 & 0.13 \\
$17302885-1639588$ & +0.05 & +0.05 & +0.05 & -0.08 & 0.04 & 0.12 \\
$17304789-1623192$ & +0.06 & +0.04 & +0.05 & -0.08 & 0.04 & 0.13 \\
$17321080-1641479$ & +0.02 & +0.07 & +0.08 & -0.09 & 0.04 & 0.15 \\
$17303589-1638299$ & +0.04 & +0.05 & +0.06 & -0.10 & 0.02 & 0.13 \\
$17290513-1630383$ & +0.02 & +0.07 & +0.07 & -0.08 & 0.05 & 0.14 \\
$17314699-1654460$ & +0.04 & +0.06 & +0.06 & -0.09 & 0.04 & 0.14 \\
$17305076-1615216$ & +0.03 & +0.06 & +0.05 & -0.10 & 0.04 & 0.14 \\
$17323324-1627474$ & +0.00 & +0.09 & +0.06 & -0.11 & 0.07 & 0.17 \\
$17312795-1631423$ & +0.03 & +0.08 & +0.05 & -0.10 & 0.04 & 0.15 \\
$17290285-1638344$ & +0.03 & +0.08 & +0.05 & -0.09 & 0.05 & 0.14 \\
$17303378-1703086$ & +0.00 & +0.09 & +0.07 & -0.11 & 0.05 & 0.17 \\
$17304223-1609274$ & +0.00 & +0.08 & +0.08 & -0.11 & 0.04 & 0.16 \\
$17304908-1633101$ & +0.01 & +0.07 & +0.07 & -0.10 & 0.04 & 0.15 \\
$17291459-1650085$ & +0.00 & +0.07 & +0.08 & -0.11 & 0.04 & 0.16 \\
$17300674-1638541$ & +0.00 & +0.07 & +0.08 & -0.11 & 0.04 & 0.16
\end{tabular}


TABLE 5A-Continued

\begin{tabular}{ccccccc}
\hline \hline Star ID & $\Delta \mathrm{T}_{\text {eff }}$ & $\Delta \log (\mathrm{g})$ & $\Delta[\mathrm{M} / \mathrm{H}]$ & $\Delta \mathrm{V}_{\mathrm{t}}$ & $\sigma / \sqrt{ }(\mathrm{N})$ & $\Delta$ Total \\
2MASS & $+100 \mathrm{~K}$ & $+0.3 \mathrm{cgs}$ & $+0.3 \mathrm{dex}$ & $+0.3 \mathrm{~km} \mathrm{~s}$ & & \\
\hline $17315010-1653413$ & +0.02 & +0.07 & +0.08 & -0.10 & 0.04 & 0.15 \\
$17295493-1613273$ & +0.00 & +0.06 & +0.09 & -0.12 & 0.04 & 0.17 \\
$17311523-1618542$ & +0.02 & +0.07 & +0.08 & -0.11 & 0.05 & 0.16 \\
$17314811-1621558$ & +0.01 & +0.09 & +0.09 & -0.11 & 0.04 & 0.17 \\
$17304861-1646459$ & -0.01 & +0.07 & +0.10 & -0.12 & 0.04 & 0.18 \\
$17323959-1625298$ & +0.01 & +0.08 & +0.09 & -0.11 & 0.04 & 0.17 \\
$17285711-1633566$ & +0.01 & +0.09 & +0.09 & -0.12 & 0.03 & 0.18 \\
$17314673-1641228$ & -0.01 & +0.07 & +0.09 & -0.11 & 0.06 & 0.17 \\
$17294476-1626118$ & -0.01 & +0.08 & +0.09 & -0.10 & 0.07 & 0.17 \\
$17320650-1643389$ & -0.02 & +0.07 & +0.07 & -0.13 & 0.05 & 0.17 \\
$17314427-1640265$ & +0.01 & +0.07 & +0.07 & -0.11 & 0.04 & 0.15 \\
$17300064-1701075$ & -0.02 & +0.07 & +0.07 & -0.13 & 0.06 & 0.18 \\
$17302935-1652277$ & +0.01 & +0.07 & +0.06 & -0.12 & 0.05 & 0.16 \\
$17320652-1619563$ & -0.01 & +0.10 & +0.06 & -0.13 & 0.05 & 0.18 \\
$17304464-1631471$ & -0.01 & +0.09 & +0.06 & -0.11 & 0.04 & 0.16 \\
$17321715-1632467$ & -0.01 & +0.09 & +0.06 & -0.12 & 0.04 & 0.17 \\
$17321969-1653156$ & -0.01 & +0.10 & +0.06 & -0.13 & 0.05 & 0.18 \\
$17315690-1630315$ & -0.02 & +0.10 & +0.06 & -0.13 & 0.03 & 0.18 \\
$17312870-1619572$ & -0.03 & +0.10 & +0.06 & -0.13 & 0.03 & 0.18 \\
$17294541-1649018$ & -0.02 & +0.09 & +0.06 & -0.14 & 0.05 & 0.18 \\
$17305067-1644006$ & -0.02 & +0.10 & +0.06 & -0.13 & 0.04 & 0.18 \\
$17312457-1652524$ & -0.01 & +0.07 & +0.06 & -0.13 & 0.04 & 0.16 \\
$17292760-1636235$ & -0.01 & +0.09 & +0.07 & -0.13 & 0.05 & 0.18 \\
$17295978-1633590$ & -0.02 & +0.11 & +0.06 & -0.13 & 0.04 & 0.19
\end{tabular}


TABLE 5A-Continued

\begin{tabular}{ccccccc}
\hline \hline Star ID & $\Delta \mathrm{T}_{\text {eff }}$ & $\Delta \log (\mathrm{g})$ & $\Delta[\mathrm{M} / \mathrm{H}]$ & $\Delta \mathrm{V}_{\mathrm{t}}$ & $\sigma / \sqrt{ }(\mathrm{N})$ & $\Delta$ Total \\
2MASS & $+100 \mathrm{~K}$ & $+0.3 \mathrm{cgs}$ & $+0.3 \mathrm{dex}$ & $+0.3 \mathrm{~km} \mathrm{~s}$ & & \\
\hline $17322323-1649335$ & -0.01 & +0.07 & +0.06 & -0.12 & 0.04 & 0.16 \\
$17321117-1654590$ & -0.01 & +0.07 & +0.06 & -0.13 & 0.03 & 0.16 \\
$17313606-1607539$ & +0.00 & +0.07 & +0.06 & -0.13 & 0.04 & 0.16 \\
$17295331-1653069$ & -0.01 & +0.07 & +0.07 & -0.14 & 0.04 & 0.18 \\
$17302818-1636032$ & -0.01 & +0.08 & +0.07 & -0.14 & 0.03 & 0.18 \\
$17293118-1638081$ & -0.01 & +0.06 & +0.07 & -0.12 & 0.04 & 0.16 \\
$17291994-1618302$ & -0.02 & +0.11 & +0.08 & -0.12 & 0.06 & 0.19 \\
$17320457-1646391$ & -0.02 & +0.09 & +0.08 & -0.13 & 0.04 & 0.18 \\
$17310747-1620049$ & -0.01 & +0.07 & +0.08 & -0.14 & 0.03 & 0.18 \\
$17323392-1636330$ & -0.03 & +0.10 & +0.08 & -0.15 & 0.04 & 0.20 \\
$17312572-1609092$ & +0.00 & +0.07 & +0.08 & -0.14 & 0.04 & 0.18 \\
$17300015-1609464$ & -0.01 & +0.07 & +0.08 & -0.14 & 0.07 & 0.19 \\
$17305894-1637056$ & -0.03 & +0.12 & +0.09 & -0.15 & 0.05 & 0.22 \\
$17291621-1647189$ & -0.03 & +0.12 & +0.09 & -0.16 & 0.03 & 0.22 \\
$17302194-1643531$ & -0.03 & +0.10 & +0.09 & -0.15 & 0.06 & 0.21 \\
$17311959-1643577$ & -0.02 & +0.08 & +0.08 & -0.15 & 0.04 & 0.19 \\
$17311229-1659047$ & -0.03 & +0.10 & +0.07 & -0.16 & 0.05 & 0.21 \\
$17313313-1659216$ & -0.02 & +0.08 & +0.07 & -0.15 & 0.04 & 0.19 \\
$17301749-1629529$ & -0.02 & +0.06 & +0.07 & -0.15 & 0.04 & 0.18 \\
$17294261-1622440$ & -0.03 & +0.07 & +0.07 & -0.16 & 0.05 & 0.20 \\
$17323625-1637551$ & -0.02 & +0.07 & +0.08 & -0.16 & 0.03 & 0.20 \\
$17314292-1620036$ & -0.03 & +0.09 & +0.09 & -0.15 & 0.06 & 0.21 \\
$17310955-1635152$ & -0.04 & +0.10 & +0.09 & -0.17 & 0.05 & 0.23 \\
$17304312-1630318$ & -0.02 & +0.07 & +0.08 & -0.16 & 0.03 & 0.20
\end{tabular}


TABle 5A-Continued

\begin{tabular}{ccccccc}
\hline \hline $\begin{array}{c}\text { Star ID } \\
\text { 2MASS }\end{array}$ & $\begin{array}{c}\Delta \mathrm{T}_{\text {eff }} \\
+100 \mathrm{~K}\end{array}$ & $\begin{array}{c}\Delta \log (\mathrm{g}) \\
+0.3 \mathrm{cgs}\end{array}$ & $\begin{array}{c}\Delta[\mathrm{M} / \mathrm{H}] \\
+0.3 \mathrm{dex}\end{array}$ & $\begin{array}{c}\Delta \mathrm{V}_{\mathrm{t}} \\
+0.3 \mathrm{~km} \mathrm{~s}^{-1}\end{array}$ & $\sigma / \sqrt{ }(\mathrm{N})$ & $\Delta$ Total \\
\hline $17285469-1627232$ & -0.01 & +0.09 & +0.08 & -0.16 & 0.03 & 0.20 \\
$17310360-1700146$ & -0.03 & +0.07 & +0.09 & -0.16 & 0.04 & 0.20 \\
$17295898-1652101$ & -0.02 & +0.08 & +0.09 & -0.17 & 0.02 & 0.21 \\
\hline
\end{tabular}


TABLE 5B

Abundance Uncertainty: Oxygen

\begin{tabular}{ccccccc}
\hline \hline Star ID & $\Delta \mathrm{T}_{\text {eff }}$ & $\begin{array}{c}\Delta \log (\mathrm{g}) \\
\text { 2MASS }\end{array}$ & $\begin{array}{c}\Delta[\mathrm{M} / \mathrm{H}] \\
+100 \mathrm{~K}\end{array}$ & $\begin{array}{c}\Delta \mathrm{V}_{\mathrm{t}} \\
+0.3 \mathrm{cgs}\end{array}$ & $\begin{array}{c}\text { dex } \\
+0.3 \mathrm{~km} \mathrm{~s}^{-1}\end{array}$ & $\Delta$ Total \\
\hline \multicolumn{7}{c}{$(\mathrm{l}, \mathrm{b})=(-5.5,-7)$} \\
\hline $18011989-3723462$ & +0.01 & +0.12 & +0.11 & -0.01 & 0.05 & 0.17 \\
$18003452-3729217$ & $\ldots$ & $\ldots$ & $\ldots$ & $\ldots$ & $\ldots$ & $\ldots$ \\
$18011692-3722508$ & +0.02 & +0.13 & +0.11 & -0.01 & 0.05 & 0.18 \\
$18005584-3739103$ & +0.01 & +0.12 & +0.11 & -0.02 & 0.05 & 0.17 \\
$18015010-3735279$ & +0.01 & +0.14 & +0.11 & -0.03 & 0.05 & 0.19 \\
$18005916-3727585$ & +0.02 & +0.12 & +0.12 & -0.02 & 0.05 & 0.18 \\
$18005027-3707505$ & +0.01 & +0.13 & +0.11 & -0.02 & 0.05 & 0.18 \\
$18005234-3715478$ & +0.02 & +0.13 & +0.12 & -0.02 & 0.05 & 0.19 \\
$18020106-3737561$ & +0.02 & +0.13 & +0.12 & -0.02 & 0.05 & 0.19 \\
$17595541-3713447$ & +0.02 & +0.12 & +0.11 & -0.02 & 0.05 & 0.17 \\
$17595664-3725304$ & +0.01 & +0.13 & +0.11 & -0.01 & 0.05 & 0.18 \\
$18021345-3730371$ & $\ldots$ & $\ldots$ & $\ldots$ & $\ldots$ & $\ldots$ & $\ldots$ \\
$17594241-3718247$ & +0.01 & +0.13 & +0.11 & -0.02 & 0.05 & 0.18 \\
$18013342-3726339$ & $\ldots$ & $\ldots$ & $\ldots$ & $\ldots$ & $\ldots$ & $\ldots$ \\
$18003204-3726008$ & +0.02 & +0.12 & +0.10 & -0.06 & 0.05 & 0.18 \\
$18013580-3740055$ & +0.02 & +0.13 & +0.11 & -0.02 & 0.05 & 0.18 \\
$18013799-3725131$ & +0.02 & +0.13 & +0.10 & -0.05 & 0.05 & 0.18 \\
$18022149-3713494$ & $\ldots$ & $\ldots$ & $\ldots$ & $\ldots$ & $\ldots$ & $\ldots$ \\
$18011408-3711299$ & +0.01 & +0.14 & +0.11 & -0.02 & 0.05 & 0.19 \\
$18021275-3737543$ & $\ldots$ & $\ldots$ & $\ldots$ & $\ldots$ & $\ldots$ & $\ldots$ \\
$17595027-3727293$ & $\ldots$ & $\ldots$ & $\ldots$ & $\ldots$ & $\ldots$ & $\ldots$ \\
$18002115-3715519$ & +0.02 & +0.12 & +0.12 & -0.04 & 0.05 & 0.18 \\
$17595366-3728186$ & $\ldots$ & $\ldots$ & $\ldots$ & $\ldots$ & $\ldots$ & $\ldots$ \\
& & & & & & $\ldots$
\end{tabular}


TABLE 5B-Continued

\begin{tabular}{|c|c|c|c|c|c|c|}
\hline $\begin{array}{l}\text { Star ID } \\
\text { 2MASS }\end{array}$ & $\begin{array}{c}\Delta \mathrm{T}_{\mathrm{eff}} \\
+100 \mathrm{~K}\end{array}$ & $\begin{array}{l}\Delta \log (\mathrm{g}) \\
+0.3 \mathrm{cgs}\end{array}$ & $\begin{array}{c}\Delta[\mathrm{M} / \mathrm{H}] \\
+0.3 \mathrm{dex}\end{array}$ & $\begin{array}{c}\Delta \mathrm{V}_{\mathrm{t}} \\
+0.3 \mathrm{~km} \mathrm{~s}^{-1}\end{array}$ & $\sigma / \sqrt{ }(\mathrm{N})$ & $\Delta$ Total \\
\hline $18001354-3709122$ & +0.02 & +0.14 & +0.12 & -0.03 & 0.05 & 0.19 \\
\hline $18013960-3723398$ & +0.02 & +0.14 & +0.12 & -0.02 & 0.05 & 0.19 \\
\hline $18021862-3734204$ & $\cdots$ & $\cdots$ & $\cdots$ & $\cdots$ & $\cdots$ & $\cdots$ \\
\hline $18000535-3735315$ & +0.02 & +0.13 & +0.12 & -0.03 & 0.05 & 0.19 \\
\hline $18013848-3721442$ & +0.02 & +0.13 & +0.12 & -0.04 & 0.05 & 0.19 \\
\hline $17594792-3721422$ & $\cdots$ & $\cdots$ & $\cdots$ & $\cdots$ & $\cdots$ & $\cdots$ \\
\hline $18011941-3721250$ & $\cdots$ & $\cdots$ & $\cdots$ & $\cdots$ & $\cdots$ & $\cdots$ \\
\hline $18004824-3718074$ & +0.02 & +0.13 & +0.12 & -0.02 & 0.05 & 0.19 \\
\hline $18014343-3708232$ & +0.02 & +0.14 & +0.12 & -0.02 & 0.05 & 0.19 \\
\hline $18005852-3711524$ & +0.01 & +0.14 & +0.12 & -0.02 & 0.05 & 0.19 \\
\hline $18020816-3714015$ & +0.02 & +0.13 & +0.12 & -0.02 & 0.05 & 0.19 \\
\hline $18010932-3702079$ & +0.02 & +0.13 & +0.13 & -0.02 & 0.05 & 0.19 \\
\hline $18010065-3740456$ & +0.02 & +0.14 & +0.13 & -0.05 & 0.05 & 0.20 \\
\hline $17595640-3710383$ & +0.02 & +0.12 & +0.12 & -0.02 & 0.05 & 0.18 \\
\hline $18012462-3738382$ & +0.02 & +0.13 & +0.11 & -0.03 & 0.05 & 0.18 \\
\hline $18002880-3710171$ & +0.01 & +0.14 & +0.12 & -0.01 & 0.05 & 0.19 \\
\hline $18024484-3714159$ & +0.01 & +0.14 & +0.12 & -0.01 & 0.05 & 0.19 \\
\hline $18013206-3708133$ & $\ldots$ & $\ldots$ & $\ldots$ & $\ldots$ & $\ldots$ & $\ldots$ \\
\hline $18004074-3740058$ & +0.02 & +0.12 & +0.11 & -0.01 & 0.05 & 0.17 \\
\hline $18000013-3715053$ & +0.02 & +0.12 & +0.11 & -0.02 & 0.05 & 0.17 \\
\hline $18015431-3717070$ & +0.02 & +0.13 & +0.11 & -0.02 & 0.05 & 0.18 \\
\hline $18021006-3738193$ & +0.01 & +0.14 & +0.12 & -0.01 & 0.05 & 0.19 \\
\hline $17595442-3721118$ & +0.02 & +0.12 & +0.11 & -0.02 & 0.05 & 0.17 \\
\hline $18021721-3736096$ & +0.01 & +0.14 & +0.12 & -0.01 & 0.05 & 0.19 \\
\hline
\end{tabular}


TABLE 5B-Continued

\begin{tabular}{ccccccc}
\hline \hline Star ID & $\Delta \mathrm{T}_{\text {eff }}$ & $\Delta \log (\mathrm{g})$ & $\Delta[\mathrm{M} / \mathrm{H}]$ & $\Delta \mathrm{V}_{\mathrm{t}}$ & $\sigma / \sqrt{ }(\mathrm{N})$ & $\Delta$ Total \\
2MASS & $+100 \mathrm{~K}$ & $+0.3 \mathrm{cgs}$ & $+0.3 \mathrm{dex}$ & $+0.3 \mathrm{~km} \mathrm{~s}$ & & \\
\hline $18005096-3733499$ & +0.02 & +0.11 & +0.10 & -0.05 & 0.05 & 0.17 \\
$18005923-3707111$ & $\ldots$ & $\ldots$ & $\ldots$ & $\ldots$ & $\ldots$ & $\ldots$ \\
$18011851-3706092$ & +0.02 & +0.12 & +0.11 & -0.03 & 0.05 & 0.17 \\
$18013395-3705551$ & +0.02 & +0.13 & +0.11 & -0.02 & 0.05 & 0.18 \\
$18022648-3728188$ & +0.01 & +0.14 & +0.12 & -0.01 & 0.05 & 0.19 \\
$18024919-3727234$ & +0.01 & +0.14 & +0.12 & -0.01 & 0.05 & 0.19 \\
$18023660-3716289$ & +0.01 & +0.14 & +0.12 & -0.02 & 0.05 & 0.19 \\
$17595509-3711099$ & $\ldots$ & $\ldots$ & $\ldots$ & $\ldots$ & $\ldots$ & $\ldots$ \\
$18005395-3731231$ & +0.02 & +0.12 & +0.12 & -0.01 & 0.05 & 0.18 \\
$18010322-3705447$ & +0.01 & +0.13 & +0.13 & -0.01 & 0.05 & 0.19 \\
$18014769-3739220$ & +0.02 & +0.14 & +0.12 & -0.01 & 0.05 & 0.19 \\
$17594807-3715283$ & +0.01 & +0.14 & +0.12 & -0.01 & 0.05 & 0.19 \\
$18013618-3706311$ & +0.02 & +0.11 & +0.12 & -0.02 & 0.05 & 0.17 \\
$18004314-3730156$ & $\ldots$ & $\ldots$ & $\ldots$ & $\ldots$ & $\ldots$ & $\ldots$ \\
$18014303-3740054$ & $\ldots$ & $\ldots$ & $\ldots$ & $\ldots$ & $\ldots$ & $\ldots$ \\
$18003751-3728200$ & +0.02 & +0.13 & +0.13 & -0.02 & 0.05 & 0.19 \\
$18011170-3727118$ & $\ldots$ & $\ldots$ & $\ldots$ & $\ldots$ & $\ldots$ & $\ldots$ \\
$18023846-3718345$ & $\ldots$ & $\ldots$ & $\ldots$ & $\ldots$ & $\ldots$ & $\ldots$ \\
$18011671-3722213$ & +0.01 & +0.14 & +0.13 & -0.01 & 0.05 & 0.20 \\
$18005985-3734507$ & $\ldots$ & $\ldots$ & $\ldots$ & $\ldots$ & $\ldots$ & $\ldots$ \\
$18000430-3711051$ & +0.02 & +0.11 & +0.12 & -0.04 & 0.05 & 0.18 \\
$18003552-3729476$ & $\ldots$ & $\ldots$ & $\ldots$ & $\ldots$ & $\ldots$ & $\ldots$ \\
$18001817-3732243$ & $\ldots$ & $\ldots$ & $\ldots$ & $\ldots$ & $\ldots$ & $\ldots$ \\
$18013039-3713086$ & +0.02 & +0.12 & +0.13 & -0.01 & 0.05 & 0.19
\end{tabular}


TABLE 5B-Continued

\begin{tabular}{ccccccc}
\hline \hline Star ID & $\begin{array}{c}\Delta \mathrm{T}_{\text {eff }} \\
\text { 2MASS }\end{array}$ & $\begin{array}{c}\Delta \log (\mathrm{g}) \\
+100 \mathrm{~K}\end{array}$ & $\begin{array}{c}\Delta[\mathrm{M} / \mathrm{H}] \\
+0.3 \mathrm{cgs}\end{array}$ & $\begin{array}{c}\Delta \mathrm{V}_{\mathrm{t}} \\
+0.3 \mathrm{~km} \mathrm{~s}\end{array}$ & $\sigma / \sqrt{ }(\mathrm{N})$ & $\Delta$ Total \\
\hline $18012564-3741052$ & +0.02 & +0.13 & +0.13 & -0.02 & 0.05 & 0.19 \\
$18025713-3718318$ & $\ldots$ & $\ldots$ & $\ldots$ & $\ldots$ & $\ldots$ & $\ldots$ \\
$18011599-3741385$ & +0.02 & +0.12 & +0.13 & -0.02 & 0.05 & 0.19 \\
$18012944-3719488$ & $\ldots$ & $\ldots$ & $\ldots$ & $\ldots$ & $\ldots$ & $\ldots$ \\
$18020361-3719086$ & +0.02 & +0.14 & +0.13 & -0.01 & 0.05 & 0.20 \\
$18024024-3728187$ & +0.02 & +0.12 & +0.13 & -0.01 & 0.05 & 0.19 \\
$18000412-3717266$ & +0.02 & +0.12 & +0.13 & -0.01 & 0.05 & 0.19 \\
$18011177-3703344$ & +0.02 & +0.12 & +0.13 & -0.01 & 0.05 & 0.19 \\
$18001410-3725181$ & +0.02 & +0.12 & +0.13 & -0.02 & 0.05 & 0.19 \\
$18020300-3719418$ & +0.02 & +0.12 & +0.12 & -0.02 & 0.05 & 0.18 \\
$18004683-3703252$ & +0.02 & +0.12 & +0.12 & -0.03 & 0.05 & 0.18 \\
$18015404-3722564$ & +0.02 & +0.12 & +0.12 & -0.04 & 0.05 & 0.18 \\
$18013974-3704068$ & +0.02 & +0.13 & +0.12 & -0.02 & 0.05 & 0.19 \\
$18020704-3708064$ & +0.02 & +0.14 & +0.13 & -0.01 & 0.05 & 0.20 \\
$18023393-3730273$ & $\ldots$ & $\ldots$ & $\ldots$ & $\ldots$ & $\ldots$ & $\ldots$ \\
$18001720-3708374$ & +0.02 & +0.12 & +0.13 & -0.01 & 0.05 & 0.19 \\
$18003663-3708547$ & $\ldots$ & $\ldots$ & $\ldots$ & $\ldots$ & $\ldots$ & $\ldots$ \\
$18002569-3729578$ & +0.02 & +0.14 & +0.13 & -0.01 & 0.05 & 0.20 \\
$17594881-3716203$ & +0.01 & +0.13 & +0.13 & -0.04 & 0.05 & 0.19 \\
$18011201-3740090$ & +0.02 & +0.14 & +0.13 & -0.01 & 0.05 & 0.20 \\
\hline & & & $(1, \mathrm{~b})=(-4,-9)$ & & & \\
\hline $18122552-3653049$ & +0.02 & +0.13 & +0.10 & -0.01 & 0.05 & 0.17 \\
$18120653-3654264$ & $\ldots$ & $\ldots$ & $\ldots$ & $\ldots$ & $\ldots$ & $\ldots$ \\
$18110179-3630394$ & +0.02 & +0.12 & +0.11 & -0.01 & 0.05 & 0.17
\end{tabular}


TABLE 5B-Continued

\begin{tabular}{ccccccc}
\hline \hline Star ID & $\Delta \mathrm{T}_{\text {eff }}$ & $\Delta \log (\mathrm{g})$ & $\Delta[\mathrm{M} / \mathrm{H}]$ & $\Delta \mathrm{V}_{\mathrm{t}}$ & $\sigma / \sqrt{ }(\mathrm{N})$ & $\Delta$ Total \\
2MASS & $+100 \mathrm{~K}$ & $+0.3 \mathrm{cgs}$ & $+0.3 \mathrm{dex}$ & $+0.3 \mathrm{~km} \mathrm{~s}{ }^{-1}$ & & \\
\hline $18124892-3626403$ & +0.01 & +0.12 & +0.10 & -0.01 & 0.05 & 0.16 \\
$18135164-3643463$ & +0.02 & +0.12 & +0.11 & -0.01 & 0.05 & 0.17 \\
$18124339-3635339$ & +0.02 & +0.12 & +0.11 & -0.01 & 0.05 & 0.17 \\
$18113921-3652069$ & $\ldots$ & $\ldots$ & $\ldots$ & $\ldots$ & $\ldots$ & $\ldots$ \\
$18111325-3646007$ & +0.02 & +0.13 & +0.12 & -0.01 & 0.05 & 0.19 \\
$18113833-3626322$ & +0.03 & +0.12 & +0.12 & -0.03 & 0.05 & 0.18 \\
$18111018-3639350$ & +0.02 & +0.13 & +0.12 & -0.01 & 0.05 & 0.19 \\
$18135243-3630481$ & +0.01 & +0.13 & +0.12 & -0.01 & 0.05 & 0.18 \\
$18121150-3627559$ & +0.02 & +0.13 & +0.12 & -0.02 & 0.05 & 0.19 \\
$18111963-3647245$ & +0.02 & +0.14 & +0.12 & -0.01 & 0.05 & 0.19 \\
$18140023-3635461$ & +0.02 & +0.14 & +0.12 & -0.02 & 0.05 & 0.19 \\
$18135881-3639345$ & +0.02 & +0.14 & +0.12 & -0.02 & 0.05 & 0.19 \\
$18121436-3654551$ & +0.02 & +0.14 & +0.11 & -0.03 & 0.05 & 0.19 \\
$18124228-3655079$ & +0.02 & +0.13 & +0.11 & -0.04 & 0.05 & 0.18 \\
$18122530-3637366$ & +0.02 & +0.14 & +0.11 & -0.02 & 0.05 & 0.19 \\
$18131370-3651584$ & $\ldots$ & $\ldots$ & $\ldots$ & $\ldots$ & $\ldots$ & $\ldots$ \\
$18134668-3623541$ & $\ldots$ & $\ldots$ & $\ldots$ & $\ldots$ & $\ldots$ & $\ldots$ \\
$18105081-3642137$ & +0.02 & +0.13 & +0.11 & -0.01 & 0.05 & 0.18 \\
$18124219-3631172$ & +0.02 & +0.13 & +0.11 & -0.02 & 0.05 & 0.18 \\
$18133688-3647054$ & $\ldots$ & $\ldots$ & $\ldots$ & $\ldots$ & $\ldots$ & $\ldots$ \\
$18123142-3639294$ & +0.02 & +0.14 & +0.10 & -0.03 & 0.05 & 0.18 \\
$18133038-3632229$ & $\ldots$ & $\ldots$ & $\ldots$ & $\ldots$ & $\ldots$ & $\ldots$ \\
$18133292-3624179$ & +0.01 & +0.14 & +0.11 & -0.01 & 0.05 & 0.19 \\
$18111681-3636318$ & +0.02 & +0.11 & +0.10 & -0.03 & 0.05 & 0.16
\end{tabular}


TABLE 5B-Continued

\begin{tabular}{ccccccc}
\hline \hline Star ID & $\Delta \mathrm{T}_{\text {eff }}$ & $\Delta \log (\mathrm{g})$ & $\Delta[\mathrm{M} / \mathrm{H}]$ & $\Delta \mathrm{V}_{\mathrm{t}}$ & $\sigma / \sqrt{ }(\mathrm{N})$ & $\Delta$ Total \\
2MASS & $+100 \mathrm{~K}$ & $+0.3 \mathrm{cgs}$ & $+0.3 \mathrm{dex}$ & $+0.3 \mathrm{~km} \mathrm{~s}$ & & \\
\hline $18105004-3640063$ & +0.02 & +0.12 & +0.11 & -0.01 & 0.05 & 0.17 \\
$18112334-3634194$ & +0.02 & +0.13 & +0.12 & -0.02 & 0.05 & 0.19 \\
$18131027-3632343$ & +0.02 & +0.13 & +0.11 & -0.02 & 0.05 & 0.18 \\
$18110293-3628041$ & +0.02 & +0.13 & +0.11 & -0.04 & 0.05 & 0.18 \\
$18132040-3623075$ & +0.01 & +0.11 & +0.11 & -0.03 & 0.05 & 0.17 \\
$18135588-3629188$ & +0.02 & +0.12 & +0.11 & -0.03 & 0.05 & 0.17 \\
$18105075-3635061$ & +0.02 & +0.13 & +0.11 & -0.03 & 0.05 & 0.18 \\
$18104188-3634541$ & +0.01 & +0.14 & +0.11 & -0.02 & 0.05 & 0.19 \\
$18132998-3628578$ & +0.02 & +0.13 & +0.11 & -0.04 & 0.05 & 0.18 \\
$18130544-3616563$ & $\ldots$ & $\ldots$ & $\ldots$ & $\ldots$ & $\ldots$ & $\ldots$ \\
$18115908-3654510$ & +0.02 & +0.14 & +0.11 & -0.02 & 0.05 & 0.19 \\
$18121077-3639094$ & +0.02 & +0.12 & +0.12 & -0.02 & 0.05 & 0.18 \\
$18120614-3629571$ & +0.01 & +0.12 & +0.12 & -0.01 & 0.05 & 0.18 \\
$18120213-3641599$ & $\ldots$ & $\ldots$ & $\ldots$ & $\ldots$ & $\ldots$ & $\ldots$ \\
$18123350-3654398$ & $\ldots$ & $\ldots$ & $\ldots$ & $\ldots$ & $\ldots$ & $\ldots$ \\
$18120973-3654184$ & +0.02 & +0.13 & +0.12 & -0.05 & 0.05 & 0.19 \\
$18105641-3632359$ & +0.02 & +0.12 & +0.12 & -0.01 & 0.05 & 0.18 \\
$18125106-3654091$ & +0.01 & +0.13 & +0.12 & -0.01 & 0.05 & 0.18 \\
$18111581-3648469$ & +0.02 & +0.13 & +0.12 & -0.02 & 0.05 & 0.19 \\
$18131387-3630130$ & $\ldots$ & $\ldots$ & $\ldots$ & $\ldots$ & $\ldots$ & $\ldots$ \\
$18123592-3634476$ & +0.03 & +0.13 & +0.13 & -0.02 & 0.05 & 0.19 \\
$18135736-3638266$ & +0.02 & +0.12 & +0.12 & -0.02 & 0.05 & 0.18 \\
$18133233-3626133$ & +0.02 & +0.13 & +0.13 & -0.03 & 0.05 & 0.19 \\
$18125003-3630269$ & +0.02 & +0.12 & +0.12 & -0.01 & 0.05 & 0.18
\end{tabular}


TABle 5B-Continued

\begin{tabular}{ccccccc}
\hline \hline Star ID & $\Delta \mathrm{T}_{\text {eff }}$ & $\Delta \log (\mathrm{g})$ & $\Delta[\mathrm{M} / \mathrm{H}]$ & $\Delta \mathrm{V}_{\mathrm{t}}$ & $\sigma / \sqrt{ }(\mathrm{N})$ & $\Delta$ Total \\
2MASS & $+100 \mathrm{~K}$ & $+0.3 \mathrm{cgs}$ & $+0.3 \mathrm{dex}$ & $+0.3 \mathrm{~km} \mathrm{~s}$ & & \\
\hline $18130723-3618385$ & +0.02 & +0.13 & +0.12 & -0.02 & 0.05 & 0.19 \\
$18125800-3616427$ & +0.02 & +0.14 & +0.12 & -0.01 & 0.05 & 0.19 \\
$18111323-3637030$ & +0.01 & +0.13 & +0.12 & -0.01 & 0.05 & 0.18 \\
$18130427-3630012$ & +0.01 & +0.14 & +0.12 & -0.01 & 0.05 & 0.19 \\
$18124614-3617326$ & $\ldots$ & $\ldots$ & $\ldots$ & $\ldots$ & $\ldots$ & $\ldots$ \\
$18120399-3615387$ & +0.02 & +0.12 & +0.12 & -0.02 & 0.05 & 0.18 \\
$18123475-3624478$ & +0.02 & +0.13 & +0.12 & -0.02 & 0.05 & 0.19 \\
$18115661-3617533$ & +0.01 & +0.14 & +0.12 & -0.01 & 0.05 & 0.19 \\
$18113299-3623341$ & +0.02 & +0.12 & +0.11 & -0.02 & 0.05 & 0.17 \\
$18111553-3628156$ & +0.02 & +0.13 & +0.11 & -0.04 & 0.05 & 0.18 \\
$18125907-3644275$ & +0.01 & +0.13 & +0.12 & -0.01 & 0.05 & 0.18 \\
$18111726-3620302$ & +0.02 & +0.13 & +0.11 & -0.01 & 0.05 & 0.18 \\
$18111579-3625114$ & +0.02 & +0.13 & +0.11 & -0.04 & 0.05 & 0.18 \\
$18131305-3650449$ & $\ldots$ & $\ldots$ & $\ldots$ & $\ldots$ & $\ldots$ & $\ldots$ \\
$18123152-3636498$ & +0.02 & +0.12 & +0.11 & -0.02 & 0.05 & 0.17 \\
$18125914-3619208$ & +0.01 & +0.14 & +0.11 & -0.01 & 0.05 & 0.19 \\
$18115073-3650493$ & $\ldots$ & $\ldots$ & $\ldots$ & $\ldots$ & $\ldots$ & $\ldots$ \\
$18114648-3625169$ & $\ldots$ & $\ldots$ & $\ldots$ & $\ldots$ & $\ldots$ & $\ldots$ \\
$18113751-3629139$ & $\ldots$ & $\ldots$ & $\ldots$ & $\ldots$ & $\ldots$ & $\ldots$ \\
$18122243-3637539$ & +0.02 & +0.12 & +0.11 & -0.02 & 0.05 & 0.17 \\
$18133366-3644575$ & +0.01 & +0.14 & +0.12 & -0.01 & 0.05 & 0.19 \\
$18134702-3641477$ & +0.01 & +0.14 & +0.12 & -0.02 & 0.05 & 0.19 \\
$18125228-3618294$ & +0.01 & +0.14 & +0.12 & +0.00 & 0.05 & 0.19 \\
$18110942-3647198$ & $\ldots$ & $\ldots$ & $\ldots$ & $\ldots$ & $\ldots$ & $\ldots$
\end{tabular}


TABLE 5B-Continued

\begin{tabular}{ccccccc}
\hline \hline Star ID & $\Delta \mathrm{T}_{\text {eff }}$ & $\Delta \log (\mathrm{g})$ & $\Delta[\mathrm{M} / \mathrm{H}]$ & $\Delta \mathrm{V}_{\mathrm{t}}$ & $\sigma / \sqrt{ }(\mathrm{N})$ & $\Delta$ Total \\
2MASS & $+100 \mathrm{~K}$ & $+0.3 \mathrm{cgs}$ & $+0.3 \mathrm{dex}$ & $+0.3 \mathrm{~km} \mathrm{~s}$ & & \\
\hline $18104824-3638507$ & +0.02 & +0.13 & +0.12 & -0.02 & 0.05 & 0.19 \\
$18122301-3629462$ & +0.02 & +0.13 & +0.12 & -0.02 & 0.05 & 0.19 \\
$18133332-3645578$ & +0.02 & +0.13 & +0.11 & -0.03 & 0.05 & 0.18 \\
$18123434-3652241$ & +0.02 & +0.12 & +0.11 & -0.03 & 0.05 & 0.17 \\
$18112311-3625456$ & +0.01 & +0.14 & +0.12 & -0.01 & 0.05 & 0.19 \\
$18125463-3649277$ & +0.02 & +0.12 & +0.11 & -0.04 & 0.05 & 0.18 \\
$18123925-3620465$ & $\ldots$ & $\ldots$ & $\ldots$ & $\ldots$ & $\ldots$ & $\ldots$ \\
$18110629-3632146$ & +0.02 & +0.12 & +0.12 & -0.01 & 0.05 & 0.18 \\
$18133687-3639590$ & +0.01 & +0.14 & +0.12 & -0.01 & 0.05 & 0.19 \\
$18111505-3620576$ & +0.01 & +0.14 & +0.13 & -0.01 & 0.05 & 0.20 \\
$18120832-3628243$ & +0.02 & +0.12 & +0.12 & -0.02 & 0.05 & 0.18 \\
$18130815-3631460$ & +0.02 & +0.13 & +0.12 & -0.02 & 0.05 & 0.19 \\
$18120407-3636481$ & +0.01 & +0.12 & +0.11 & -0.03 & 0.05 & 0.17 \\
$18135786-3640300$ & +0.02 & +0.14 & +0.12 & -0.01 & 0.05 & 0.19 \\
$18131228-3619138$ & +0.02 & +0.13 & +0.13 & -0.01 & 0.05 & 0.19 \\
$18113372-3650200$ & +0.02 & +0.12 & +0.12 & -0.02 & 0.05 & 0.18 \\
$18115904-3650428$ & $\ldots$ & $\ldots$ & $\ldots$ & $\ldots$ & $\ldots$ & $\ldots$ \\
$18115448-3641161$ & +0.02 & +0.13 & +0.13 & -0.01 & 0.05 & 0.19 \\
$18123678-3649019$ & +0.02 & +0.13 & +0.12 & -0.03 & 0.05 & 0.19 \\
$18113965-3645408$ & $\ldots$ & $\ldots$ & $\ldots$ & $\ldots$ & $\ldots$ & $\ldots$ \\
$18135362-3636538$ & $\ldots$ & $\ldots$ & $\ldots$ & $\ldots$ & $\ldots$ & $\ldots$ \\
$18123168-3631555$ & +0.01 & +0.13 & +0.13 & -0.01 & 0.05 & 0.19 \\
$18115398-3623290$ & +0.01 & +0.12 & +0.13 & -0.05 & 0.05 & 0.19 \\
$18123393-3615508$ & $\ldots$ & $\ldots$ & $\ldots$ & $\ldots$ & $\ldots$ & $\ldots$
\end{tabular}


TABLE 5B-Continued

\begin{tabular}{ccccccc}
\hline \hline Star ID & $\begin{array}{c}\Delta \mathrm{T}_{\text {eff }} \\
\text { 2MASS }\end{array}$ & $\begin{array}{c}\Delta \log (\mathrm{g}) \\
+0.3 \mathrm{cgs}\end{array}$ & $\begin{array}{c}\Delta[\mathrm{M} / \mathrm{H}] \\
+0.3 \mathrm{dex}\end{array}$ & $\begin{array}{c}\Delta \mathrm{V}_{\mathrm{t}} \\
+0.3 \mathrm{~km} \mathrm{~s}\end{array}$ & $\sigma / \sqrt{ }(\mathrm{N})$ & $\Delta$ Total \\
\hline $18120689-3643231$ & +0.02 & +0.14 & +0.13 & -0.01 & 0.05 & 0.20 \\
$18130482-3649019$ & +0.02 & +0.12 & +0.13 & -0.02 & 0.05 & 0.19 \\
$18110352-3646275$ & +0.02 & +0.13 & +0.13 & -0.02 & 0.05 & 0.19 \\
$18105013-3629557$ & +0.02 & +0.13 & +0.13 & -0.01 & 0.05 & 0.19 \\
$18121897-3630243$ & $\ldots$ & $\ldots$ & $\ldots$ & $\ldots$ & $\ldots$ & $\ldots$ \\
$18122029-3652333$ & $\ldots$ & $\ldots$ & $\ldots$ & $\ldots$ & $\ldots$ & $\ldots$ \\
\hline & & $(1, \mathrm{~b})=(+8.5,+9)$ & & & \\
\hline $17311256-1702018$ & +0.02 & +0.12 & +0.11 & -0.03 & 0.05 & 0.17 \\
$17290874-1632431$ & +0.01 & +0.13 & +0.11 & -0.03 & 0.05 & 0.18 \\
$17302885-1639588$ & +0.02 & +0.12 & +0.12 & -0.02 & 0.05 & 0.18 \\
$17304789-1623192$ & +0.02 & +0.11 & +0.12 & -0.02 & 0.05 & 0.17 \\
$17321080-1641479$ & +0.02 & +0.12 & +0.12 & -0.04 & 0.05 & 0.18 \\
$17303589-1638299$ & +0.03 & +0.12 & +0.12 & -0.03 & 0.05 & 0.18 \\
$17290513-1630383$ & +0.03 & +0.12 & +0.12 & -0.04 & 0.05 & 0.18 \\
$17314699-1654460$ & +0.02 & +0.13 & +0.11 & -0.04 & 0.05 & 0.18 \\
$17305076-1615216$ & +0.02 & +0.12 & +0.10 & -0.04 & 0.05 & 0.17 \\
$17323324-1627474$ & +0.02 & +0.13 & +0.11 & -0.03 & 0.05 & 0.18 \\
$17312795-1631423$ & +0.02 & +0.14 & +0.11 & -0.02 & 0.05 & 0.19 \\
$17290285-1638344$ & +0.02 & +0.14 & +0.11 & -0.02 & 0.05 & 0.19 \\
$17303378-1703086$ & +0.03 & +0.13 & +0.11 & -0.04 & 0.05 & 0.18 \\
$17304223-1609274$ & +0.02 & +0.13 & +0.12 & -0.04 & 0.05 & 0.19 \\
$17304908-1633101$ & $\ldots$ & $\ldots$ & $\ldots$ & $\ldots$ & $\ldots$ & $\ldots$ \\
$17291459-1650085$ & +0.03 & +0.12 & +0.12 & -0.03 & 0.05 & 0.18 \\
$17300674-1638541$ & $\ldots$ & $\ldots$ & $\ldots$ & $\ldots$ & $\ldots$ & $\ldots$ \\
& & & & & & $\ldots$
\end{tabular}


TABle 5B-Continued

\begin{tabular}{ccccccc}
\hline \hline Star ID & $\Delta \mathrm{T}_{\text {eff }}$ & $\Delta \log (\mathrm{g})$ & $\Delta[\mathrm{M} / \mathrm{H}]$ & $\Delta \mathrm{V}_{\mathrm{t}}$ & $\sigma / \sqrt{ }(\mathrm{N})$ & $\Delta$ Total \\
2MASS & $+100 \mathrm{~K}$ & $+0.3 \mathrm{cgs}$ & $+0.3 \mathrm{dex}$ & $+0.3 \mathrm{~km} \mathrm{~s}$ & & \\
\hline $17315010-1653413$ & +0.02 & +0.12 & +0.12 & -0.03 & 0.05 & 0.18 \\
$17295493-1613273$ & $\ldots$ & $\ldots$ & $\ldots$ & $\ldots$ & $\ldots$ & $\ldots$ \\
$17311523-1618542$ & $\ldots$ & $\ldots$ & $\ldots$ & $\ldots$ & $\ldots$ & $\ldots$ \\
$17314811-1621558$ & +0.02 & +0.13 & +0.12 & -0.02 & 0.05 & 0.19 \\
$17304861-1646459$ & +0.03 & +0.11 & +0.14 & -0.04 & 0.05 & 0.19 \\
$17323959-1625298$ & $\ldots$ & $\ldots$ & $\ldots$ & $\ldots$ & $\ldots$ & $\ldots$ \\
$17285711-1633566$ & +0.03 & +0.13 & +0.13 & -0.03 & 0.05 & 0.20 \\
$17314673-1641228$ & +0.03 & +0.11 & +0.13 & -0.03 & 0.05 & 0.18 \\
$17294476-1626118$ & +0.02 & +0.12 & +0.13 & -0.03 & 0.05 & 0.19 \\
$17320650-1643389$ & +0.02 & +0.12 & +0.12 & -0.03 & 0.05 & 0.18 \\
$17314427-1640265$ & +0.02 & +0.12 & +0.11 & -0.03 & 0.05 & 0.17 \\
$17300064-1701075$ & $\ldots$ & $\ldots$ & $\ldots$ & $\ldots$ & $\ldots$ & $\ldots$ \\
$17302935-1652277$ & +0.02 & +0.12 & +0.12 & -0.01 & 0.05 & 0.18 \\
$17320652-1619563$ & +0.02 & +0.14 & +0.11 & -0.02 & 0.05 & 0.19 \\
$17304464-1631471$ & +0.02 & +0.13 & +0.10 & -0.05 & 0.05 & 0.18 \\
$17321715-1632467$ & $\ldots$ & $\ldots$ & $\ldots$ & $\ldots$ & $\ldots$ & $\ldots$ \\
$17321969-1653156$ & +0.02 & +0.14 & +0.10 & -0.05 & 0.05 & 0.19 \\
$17315690-1630315$ & +0.02 & +0.13 & +0.12 & -0.02 & 0.05 & 0.19 \\
$17312870-1619572$ & +0.02 & +0.13 & +0.12 & -0.03 & 0.05 & 0.19 \\
$17294541-1649018$ & +0.02 & +0.13 & +0.11 & -0.04 & 0.05 & 0.18 \\
$17305067-1644006$ & +0.02 & +0.14 & +0.12 & -0.03 & 0.05 & 0.19 \\
$17312457-1652524$ & +0.02 & +0.12 & +0.11 & -0.04 & 0.05 & 0.18 \\
$17292760-1636235$ & +0.02 & +0.13 & +0.11 & -0.06 & 0.05 & 0.19 \\
$17295978-1633590$ & $\ldots$ & $\ldots$ & $\ldots$ & $\ldots$ & $\ldots$ & $\ldots$
\end{tabular}


TABle 5B-Continued

\begin{tabular}{ccccccc}
\hline \hline Star ID & $\Delta \mathrm{T}_{\text {eff }}$ & $\Delta \log (\mathrm{g})$ & $\Delta[\mathrm{M} / \mathrm{H}]$ & $\Delta \mathrm{V}_{\mathrm{t}}$ & $\sigma / \sqrt{ }(\mathrm{N})$ & $\Delta$ Total \\
2MASS & $+100 \mathrm{~K}$ & $+0.3 \mathrm{cgs}$ & $+0.3 \mathrm{dex}$ & $+0.3 \mathrm{~km} \mathrm{~s}$ & & \\
\hline $17322323-1649335$ & +0.02 & +0.12 & +0.11 & -0.03 & 0.05 & 0.17 \\
$17321117-1654590$ & +0.02 & +0.12 & +0.12 & -0.02 & 0.05 & 0.18 \\
$17313606-1607539$ & +0.02 & +0.12 & +0.11 & -0.03 & 0.05 & 0.17 \\
$17295331-1653069$ & +0.02 & +0.12 & +0.11 & -0.04 & 0.05 & 0.18 \\
$17302818-1636032$ & +0.02 & +0.13 & +0.11 & -0.03 & 0.05 & 0.18 \\
$17293118-1638081$ & $\ldots$ & $\ldots$ & $\ldots$ & $\ldots$ & $\ldots$ & $\ldots$ \\
$17291994-1618302$ & +0.02 & +0.14 & +0.12 & -0.03 & 0.05 & 0.19 \\
$17320457-1646391$ & +0.02 & +0.13 & +0.12 & -0.02 & 0.05 & 0.19 \\
$17310747-1620049$ & +0.02 & +0.12 & +0.12 & -0.04 & 0.05 & 0.18 \\
$17323392-1636330$ & +0.02 & +0.13 & +0.13 & -0.02 & 0.05 & 0.19 \\
$17312572-1609092$ & +0.02 & +0.12 & +0.13 & -0.01 & 0.05 & 0.19 \\
$17300015-1609464$ & $\ldots$ & $\ldots$ & $\ldots$ & $\ldots$ & $\ldots$ & $\ldots$ \\
$17305894-1637056$ & +0.01 & +0.15 & +0.13 & -0.05 & 0.05 & 0.21 \\
$17291621-1647189$ & +0.02 & +0.15 & +0.13 & -0.04 & 0.05 & 0.21 \\
$17302194-1643531$ & +0.01 & +0.14 & +0.12 & -0.04 & 0.05 & 0.20 \\
$17311959-1643577$ & $\ldots$ & $\ldots$ & $\ldots$ & $\ldots$ & $\ldots$ & $\ldots$ \\
$17311229-1659047$ & $\ldots$ & $\ldots$ & $\ldots$ & $\ldots$ & $\ldots$ & $\ldots$ \\
$17313313-1659216$ & +0.02 & +0.12 & +0.12 & -0.03 & 0.05 & 0.18 \\
$17301749-1629529$ & +0.02 & +0.12 & +0.12 & -0.02 & 0.05 & 0.18 \\
$17294261-1622440$ & $\ldots$ & $\ldots$ & $\ldots$ & $\ldots$ & $\ldots$ & $\ldots$ \\
$17323625-1637551$ & +0.02 & +0.12 & +0.13 & -0.01 & 0.05 & 0.19 \\
$17314292-1620036$ & $\ldots$ & $\ldots$ & $\ldots$ & $\ldots$ & $\ldots$ & $\ldots$ \\
$17310955-1635152$ & +0.02 & +0.13 & +0.13 & -0.02 & 0.05 & 0.19 \\
$17304312-1630318$ & $\ldots$ & $\ldots$ & $\ldots$ & $\ldots$ & $\ldots$ & $\ldots$
\end{tabular}


TABle 5B-Continued

\begin{tabular}{ccccccc}
\hline \hline $\begin{array}{c}\text { Star ID } \\
\text { 2MASS }\end{array}$ & $\begin{array}{c}\Delta \mathrm{T}_{\text {eff }} \\
+100 \mathrm{~K}\end{array}$ & $\begin{array}{c}\Delta \log (\mathrm{g}) \\
+0.3 \mathrm{cgs}\end{array}$ & $\begin{array}{c}\Delta[\mathrm{M} / \mathrm{H}] \\
+0.3 \mathrm{dex}\end{array}$ & $\begin{array}{c}\Delta \mathrm{V}_{\mathrm{t}} \\
+0.3 \mathrm{~km} \mathrm{~s}^{-1}\end{array}$ & $\sigma / \sqrt{ }(\mathrm{N})$ & $\Delta$ Total \\
\hline $17285469-1627232$ & +0.02 & +0.13 & +0.13 & -0.02 & 0.05 & 0.19 \\
$17310360-1700146$ & $\ldots$ & $\ldots$ & $\ldots$ & $\ldots$ & $\ldots$ & $\ldots$ \\
$17295898-1652101$ & +0.01 & +0.13 & +0.13 & -0.02 & 0.05 & 0.19 \\
\hline
\end{tabular}


TABLE 5C

Abundance Uncertainty: Silicon

\begin{tabular}{ccccccc}
\hline \hline Star ID & $\begin{array}{c}\Delta \mathrm{T}_{\text {eff }} \\
\text { 2MASS }\end{array}$ & $\begin{array}{c}\Delta \log (\mathrm{g}) \\
+100 \mathrm{~K}\end{array}$ & $\begin{array}{c}\Delta[\mathrm{M} / \mathrm{H}] \\
+0.3 \mathrm{dex}\end{array}$ & $\begin{array}{c}\Delta \mathrm{V}_{\mathrm{t}} \\
+0.3 \mathrm{~km} \mathrm{~s}\end{array}$ & $\sigma / \sqrt{ }(\mathrm{N})$ & $\Delta$ Total \\
\hline \multicolumn{7}{c}{$(\mathrm{l}, \mathrm{b})=(-5.5,-7)$} \\
\hline $18011989-3723462$ & -0.02 & +0.03 & +0.04 & -0.01 & 0.05 & 0.07 \\
$18003452-3729217$ & +0.00 & +0.04 & +0.03 & -0.01 & 0.05 & 0.07 \\
$18011692-3722508$ & +0.00 & +0.06 & +0.04 & -0.01 & 0.05 & 0.09 \\
$18005584-3739103$ & $\ldots$ & $\ldots$ & $\ldots$ & $\ldots$ & $\ldots$ & $\ldots$ \\
$18015010-3735279$ & -0.03 & +0.08 & +0.05 & -0.02 & 0.12 & 0.16 \\
$18005916-3727585$ & -0.02 & +0.04 & +0.06 & -0.02 & 0.05 & 0.09 \\
$18005027-3707505$ & -0.02 & +0.06 & +0.05 & -0.02 & 0.03 & 0.09 \\
$18005234-3715478$ & -0.05 & +0.07 & +0.06 & -0.02 & 0.05 & 0.12 \\
$18020106-3737561$ & -0.05 & +0.08 & +0.06 & -0.02 & 0.05 & 0.12 \\
$17595541-3713447$ & $\ldots$ & $\ldots$ & $\ldots$ & $\ldots$ & $\ldots$ & $\ldots$ \\
$17595664-3725304$ & -0.03 & +0.06 & +0.05 & -0.03 & 0.05 & 0.10 \\
$18021345-3730371$ & -0.08 & +0.09 & +0.04 & -0.03 & 0.03 & 0.13 \\
$17594241-3718247$ & -0.06 & +0.07 & +0.05 & -0.03 & 0.05 & 0.12 \\
$18013342-3726339$ & -0.04 & +0.05 & +0.05 & -0.03 & 0.05 & 0.10 \\
$18003204-3726008$ & -0.09 & +0.07 & +0.05 & -0.03 & 0.00 & 0.13 \\
$18013580-3740055$ & -0.09 & +0.09 & +0.05 & -0.03 & 0.06 & 0.15 \\
$18013799-3725131$ & -0.09 & +0.09 & +0.05 & -0.04 & 0.02 & 0.14 \\
$18022149-3713494$ & -0.08 & +0.10 & +0.05 & -0.03 & 0.05 & 0.15 \\
$18011408-3711299$ & -0.04 & +0.08 & +0.06 & -0.04 & 0.01 & 0.12 \\
$18021275-3737543$ & $\ldots$ & $\ldots$ & $\ldots$ & $\ldots$ & $\ldots$ & $\ldots$ \\
$17595027-3727293$ & -0.07 & +0.08 & +0.06 & -0.03 & 0.05 & 0.14 \\
$18002115-3715519$ & -0.10 & +0.08 & +0.07 & -0.03 & 0.04 & 0.15 \\
$17595366-3728186$ & -0.04 & +0.07 & +0.07 & -0.04 & 0.05 & 0.12
\end{tabular}


TABLE 5C-Continued

\begin{tabular}{ccccccc}
\hline \hline Star ID & $\Delta \mathrm{T}_{\text {eff }}$ & $\Delta \log (\mathrm{g})$ & $\Delta[\mathrm{M} / \mathrm{H}]$ & $\Delta \mathrm{V}_{\mathrm{t}}$ & $\sigma / \sqrt{ }(\mathrm{N})$ & $\Delta$ Total \\
2MASS & $+100 \mathrm{~K}$ & $+0.3 \mathrm{cgs}$ & $+0.3 \mathrm{dex}$ & $+0.3 \mathrm{~km} \mathrm{~s}$ & & \\
\hline $18001354-3709122$ & -0.09 & +0.13 & +0.08 & -0.04 & 0.05 & 0.19 \\
$18013960-3723398$ & -0.09 & +0.12 & +0.07 & -0.04 & 0.08 & 0.19 \\
$18021862-3734204$ & $\ldots$ & $\ldots$ & $\ldots$ & $\ldots$ & $\ldots$ & $\ldots$ \\
$18000535-3735315$ & -0.09 & +0.10 & +0.07 & -0.04 & 0.02 & 0.16 \\
$18013848-3721442$ & -0.08 & +0.11 & +0.08 & -0.03 & 0.05 & 0.17 \\
$17594792-3721422$ & -0.07 & +0.05 & +0.07 & -0.04 & 0.01 & 0.12 \\
$18011941-3721250$ & $\ldots$ & $\ldots$ & $\ldots$ & $\ldots$ & $\ldots$ & $\ldots$ \\
$18004824-3718074$ & -0.10 & +0.10 & +0.08 & -0.03 & 0.05 & 0.17 \\
$18014343-3708232$ & -0.09 & +0.12 & +0.08 & -0.03 & 0.10 & 0.20 \\
$18005852-3711524$ & -0.04 & +0.08 & +0.07 & -0.05 & 0.02 & 0.13 \\
$18020816-3714015$ & -0.04 & +0.07 & +0.07 & -0.04 & 0.14 & 0.18 \\
$18010932-3702079$ & -0.09 & +0.12 & +0.08 & -0.03 & 0.19 & 0.26 \\
$18010065-3740456$ & -0.09 & +0.12 & +0.09 & -0.04 & 0.05 & 0.19 \\
$17595640-3710383$ & -0.08 & +0.05 & +0.06 & -0.04 & 0.05 & 0.13 \\
$18012462-3738382$ & -0.05 & +0.08 & +0.07 & -0.04 & 0.05 & 0.13 \\
$18002880-3710171$ & -0.04 & +0.09 & +0.05 & -0.04 & 0.05 & 0.13 \\
$18024484-3714159$ & -0.04 & +0.09 & +0.05 & -0.03 & 0.05 & 0.12 \\
$18013206-3708133$ & $\ldots$ & $\ldots$ & $\ldots$ & $\ldots$ & $\ldots$ & $\ldots$ \\
$18004074-3740058$ & -0.07 & +0.06 & +0.05 & -0.03 & 0.05 & 0.12 \\
$18000013-3715053$ & -0.08 & +0.07 & +0.04 & -0.04 & 0.02 & 0.12 \\
$18015431-3717070$ & -0.06 & +0.09 & +0.05 & -0.04 & 0.13 & 0.18 \\
$18021006-3738193$ & -0.04 & +0.08 & +0.05 & -0.03 & 0.08 & 0.13 \\
$17595442-3721118$ & -0.09 & +0.09 & +0.03 & -0.05 & 0.14 & 0.20 \\
$18021721-3736096$ & -0.04 & +0.10 & +0.05 & -0.03 & 0.05 & 0.13
\end{tabular}


TABLE 5C-Continued

\begin{tabular}{ccccccc}
\hline \hline Star ID & $\Delta \mathrm{T}_{\text {eff }}$ & $\Delta \log (\mathrm{g})$ & $\Delta[\mathrm{M} / \mathrm{H}]$ & $\Delta \mathrm{V}_{\mathrm{t}}$ & $\sigma / \sqrt{ }(\mathrm{N})$ & $\Delta$ Total \\
2MASS & $+100 \mathrm{~K}$ & $+0.3 \mathrm{cgs}$ & $+0.3 \mathrm{dex}$ & $+0.3 \mathrm{~km} \mathrm{~s}$ & & \\
\hline $18005096-3733499$ & -0.09 & +0.05 & +0.04 & -0.05 & 0.05 & 0.13 \\
$18005923-3707111$ & -0.10 & +0.07 & +0.03 & -0.04 & 0.09 & 0.16 \\
$18011851-3706092$ & -0.10 & +0.07 & +0.03 & -0.04 & 0.00 & 0.13 \\
$18013395-3705551$ & -0.10 & +0.09 & +0.03 & -0.05 & 0.02 & 0.15 \\
$18022648-3728188$ & -0.05 & +0.10 & +0.05 & -0.04 & 0.26 & 0.29 \\
$18024919-3727234$ & -0.05 & +0.10 & +0.05 & -0.04 & 0.14 & 0.19 \\
$18023660-3716289$ & -0.05 & +0.10 & +0.05 & -0.06 & 0.05 & 0.15 \\
$17595509-3711099$ & -0.07 & +0.08 & +0.05 & -0.03 & 0.05 & 0.13 \\
$18005395-3731231$ & -0.10 & +0.10 & +0.03 & -0.04 & 0.06 & 0.16 \\
$18010322-3705447$ & -0.05 & +0.09 & +0.05 & -0.04 & 0.05 & 0.13 \\
$18014769-3739220$ & $\ldots$ & $\ldots$ & $\ldots$ & $\ldots$ & $\ldots$ & $\ldots$ \\
$17594807-3715283$ & -0.05 & +0.11 & +0.05 & -0.04 & 0.05 & 0.15 \\
$18013618-3706311$ & -0.09 & +0.05 & +0.05 & -0.05 & 0.04 & 0.13 \\
$18004314-3730156$ & -0.05 & +0.11 & +0.07 & -0.04 & 0.02 & 0.15 \\
$18014303-3740054$ & -0.08 & +0.07 & +0.06 & -0.04 & 0.05 & 0.14 \\
$18003751-3728200$ & -0.10 & +0.12 & +0.06 & -0.04 & 0.02 & 0.17 \\
$18011170-3727118$ & -0.03 & +0.08 & +0.06 & -0.03 & 0.05 & 0.12 \\
$18023846-3718345$ & -0.08 & +0.09 & +0.07 & -0.03 & 0.05 & 0.15 \\
$18011671-3722213$ & -0.04 & +0.08 & +0.07 & -0.04 & 0.05 & 0.13 \\
$18005985-3734507$ & $\ldots$ & $\ldots$ & $\ldots$ & $\ldots$ & $\ldots$ & $\ldots$ \\
$18000430-3711051$ & -0.09 & +0.06 & +0.07 & -0.05 & 0.08 & 0.16 \\
$18003552-3729476$ & -0.08 & +0.08 & +0.07 & -0.04 & 0.00 & 0.14 \\
$18001817-3732243$ & -0.06 & +0.10 & +0.07 & -0.05 & 0.05 & 0.15 \\
$18013039-3713086$ & -0.09 & +0.09 & +0.07 & -0.04 & 0.01 & 0.15 \\
& & & & & &
\end{tabular}


TABLE 5C-Continued

\begin{tabular}{ccccccc}
\hline \hline Star ID & $\begin{array}{c}\Delta \mathrm{T}_{\text {eff }} \\
\text { 2MASS }\end{array}$ & $\begin{array}{c}\Delta \log (\mathrm{g}) \\
+0.3 \mathrm{cgs}\end{array}$ & $\begin{array}{c}\Delta[\mathrm{M} / \mathrm{H}] \\
+0.3 \mathrm{dex}\end{array}$ & $\begin{array}{c}\Delta \mathrm{V}_{\mathrm{t}} \\
+0.3 \mathrm{~km} \mathrm{~s}\end{array}$ & $\sigma / \sqrt{ }(\mathrm{N})$ & $\Delta$ Total \\
\hline $18012564-3741052$ & -0.08 & +0.10 & +0.07 & -0.04 & 0.09 & 0.18 \\
$18025713-3718318$ & -0.09 & +0.10 & +0.06 & -0.04 & 0.06 & 0.16 \\
$18011599-3741385$ & -0.09 & +0.08 & +0.07 & -0.06 & 0.06 & 0.16 \\
$18012944-3719488$ & -0.04 & +0.09 & +0.06 & -0.04 & 0.05 & 0.13 \\
$18020361-3719086$ & -0.07 & +0.11 & +0.07 & -0.06 & 0.05 & 0.17 \\
$18024024-3728187$ & -0.10 & +0.07 & +0.06 & -0.04 & 0.04 & 0.15 \\
$18000412-3717266$ & -0.10 & +0.06 & +0.05 & -0.04 & 0.02 & 0.13 \\
$18011177-3703344$ & -0.08 & +0.09 & +0.06 & -0.03 & 0.14 & 0.20 \\
$18001410-3725181$ & -0.10 & +0.08 & +0.05 & -0.04 & 0.11 & 0.18 \\
$18020300-3719418$ & -0.10 & +0.06 & +0.04 & -0.04 & 0.12 & 0.18 \\
$18004683-3703252$ & -0.10 & +0.09 & +0.04 & -0.04 & 0.15 & 0.21 \\
$18015404-3722564$ & -0.08 & +0.08 & +0.04 & -0.03 & 0.02 & 0.13 \\
$18013974-3704068$ & -0.08 & +0.09 & +0.06 & -0.04 & 0.08 & 0.16 \\
$18020704-3708064$ & -0.05 & +0.11 & +0.06 & -0.04 & 0.05 & 0.15 \\
$18023393-3730273$ & $\ldots$ & $\ldots$ & $\ldots$ & $\ldots$ & $\ldots$ & $\ldots$ \\
$18001720-3708374$ & -0.10 & +0.07 & +0.06 & -0.03 & 0.05 & 0.15 \\
$18003663-3708547$ & -0.08 & +0.10 & +0.06 & -0.04 & 0.00 & 0.15 \\
$18002569-3729578$ & -0.06 & +0.13 & +0.06 & -0.04 & 0.05 & 0.17 \\
$17594881-3716203$ & -0.09 & +0.12 & +0.06 & -0.04 & 0.02 & 0.17 \\
$18011201-3740090$ & -0.06 & +0.13 & +0.06 & -0.06 & 0.05 & 0.17 \\
\hline & & & $(1, \mathrm{~b})=(-4,-9)$ & & & \\
\hline $18122552-3653049$ & +0.01 & +0.04 & +0.03 & -0.01 & 0.05 & 0.07 \\
$18120653-3654264$ & +0.00 & +0.03 & +0.03 & -0.01 & 0.05 & 0.07 \\
$18110179-3630394$ & +0.00 & +0.04 & +0.04 & -0.01 & 0.05 & 0.08
\end{tabular}


TABLE 5C-Continued

\begin{tabular}{ccccccc}
\hline \hline Star ID & $\Delta \mathrm{T}_{\text {eff }}$ & $\Delta \log (\mathrm{g})$ & $\Delta[\mathrm{M} / \mathrm{H}]$ & $\Delta \mathrm{V}_{\mathrm{t}}$ & $\sigma / \sqrt{ }(\mathrm{N})$ & $\Delta$ Total \\
2MASS & $+100 \mathrm{~K}$ & $+0.3 \mathrm{cgs}$ & $+0.3 \mathrm{dex}$ & $+0.3 \mathrm{~km} \mathrm{~s}$ & & \\
\hline $18124892-3626403$ & -0.01 & +0.04 & +0.03 & -0.01 & 0.05 & 0.07 \\
$18135164-3643463$ & -0.01 & +0.04 & +0.04 & -0.01 & 0.02 & 0.06 \\
$18124339-3635339$ & -0.01 & +0.05 & +0.04 & -0.02 & 0.05 & 0.08 \\
$18113921-3652069$ & -0.04 & +0.07 & +0.05 & -0.02 & 0.05 & 0.11 \\
$18111325-3646007$ & -0.01 & +0.05 & +0.05 & -0.02 & 0.15 & 0.17 \\
$18113833-3626322$ & -0.05 & +0.07 & +0.07 & -0.03 & 0.05 & 0.13 \\
$18111018-3639350$ & -0.05 & +0.07 & +0.06 & -0.02 & 0.05 & 0.12 \\
$18135243-3630481$ & -0.02 & +0.04 & +0.05 & -0.02 & 0.05 & 0.09 \\
$18121150-3627559$ & -0.04 & +0.06 & +0.06 & -0.02 & 0.05 & 0.11 \\
$18111963-3647245$ & -0.05 & +0.08 & +0.06 & -0.03 & 0.03 & 0.12 \\
$18140023-3635461$ & -0.06 & +0.09 & +0.07 & -0.05 & 0.02 & 0.14 \\
$18135881-3639345$ & -0.05 & +0.09 & +0.06 & -0.02 & 0.05 & 0.13 \\
$18121436-3654551$ & -0.07 & +0.10 & +0.07 & -0.05 & 0.05 & 0.16 \\
$18124228-3655079$ & -0.07 & +0.08 & +0.07 & -0.04 & 0.05 & 0.14 \\
$18122530-3637366$ & -0.06 & +0.11 & +0.06 & -0.04 & 0.13 & 0.19 \\
$18131370-3651584$ & -0.06 & +0.08 & +0.05 & -0.04 & 0.05 & 0.13 \\
$18134668-3623541$ & $\ldots$ & $\ldots$ & $\ldots$ & $\ldots$ & $\ldots$ & $\ldots$ \\
$18105081-3642137$ & -0.03 & +0.05 & +0.05 & -0.03 & 0.05 & 0.10 \\
$18124219-3631172$ & -0.07 & +0.09 & +0.06 & -0.04 & 0.00 & 0.13 \\
$18133688-3647054$ & $\ldots$ & $\ldots$ & $\ldots$ & $\ldots$ & $\ldots$ & $\ldots$ \\
$18123142-3639294$ & -0.08 & +0.11 & +0.04 & -0.03 & 0.05 & 0.15 \\
$18133038-3632229$ & -0.03 & +0.06 & +0.04 & -0.03 & 0.05 & 0.10 \\
$18133292-3624179$ & $\ldots$ & $\ldots$ & $\ldots$ & $\ldots$ & $\ldots$ & $\ldots$ \\
$18111681-3636318$ & -0.09 & +0.06 & +0.04 & -0.03 & 0.05 & 0.13
\end{tabular}


TABLE 5C-Continued

\begin{tabular}{ccccccc}
\hline \hline Star ID & $\Delta \mathrm{T}_{\text {eff }}$ & $\Delta \log (\mathrm{g})$ & $\Delta[\mathrm{M} / \mathrm{H}]$ & $\Delta \mathrm{V}_{\mathrm{t}}$ & $\sigma / \sqrt{ }(\mathrm{N})$ & $\Delta$ Total \\
2MASS & $+100 \mathrm{~K}$ & $+0.3 \mathrm{cgs}$ & $+0.3 \mathrm{dex}$ & $+0.3 \mathrm{~km} \mathrm{~s}$ & & \\
\hline $18105004-3640063$ & -0.04 & +0.05 & +0.05 & -0.03 & 0.05 & 0.10 \\
$18112334-3634194$ & -0.05 & +0.06 & +0.06 & -0.03 & 0.08 & 0.13 \\
$18131027-3632343$ & -0.07 & +0.08 & +0.05 & -0.04 & 0.04 & 0.13 \\
$18110293-3628041$ & -0.09 & +0.08 & +0.05 & -0.05 & 0.11 & 0.18 \\
$18132040-3623075$ & -0.07 & +0.05 & +0.06 & -0.03 & 0.07 & 0.13 \\
$18135588-3629188$ & -0.05 & +0.06 & +0.06 & -0.03 & 0.03 & 0.11 \\
$18105075-3635061$ & -0.09 & +0.10 & +0.06 & -0.04 & 0.11 & 0.19 \\
$18104188-3634541$ & -0.03 & +0.07 & +0.05 & -0.03 & 0.05 & 0.11 \\
$18132998-3628578$ & -0.09 & +0.10 & +0.06 & -0.03 & 0.00 & 0.15 \\
$18130544-3616563$ & -0.08 & +0.10 & +0.06 & -0.05 & 0.05 & 0.16 \\
$18115908-3654510$ & -0.08 & +0.12 & +0.07 & -0.05 & 0.05 & 0.18 \\
$18121077-3639094$ & -0.08 & +0.07 & +0.07 & -0.04 & 0.05 & 0.14 \\
$18120614-3629571$ & -0.06 & +0.05 & +0.07 & -0.04 & 0.05 & 0.12 \\
$18120213-3641599$ & -0.04 & +0.07 & +0.07 & -0.05 & 0.05 & 0.13 \\
$18123350-3654398$ & -0.10 & +0.10 & +0.08 & -0.06 & 0.09 & 0.20 \\
$18120973-3654184$ & -0.11 & +0.10 & +0.09 & -0.06 & 0.05 & 0.19 \\
$18105641-3632359$ & -0.08 & +0.04 & +0.08 & -0.05 & 0.05 & 0.14 \\
$18125106-3654091$ & -0.04 & +0.07 & +0.07 & -0.04 & 0.10 & 0.15 \\
$18111581-3648469$ & -0.10 & +0.10 & +0.09 & -0.04 & 0.11 & 0.20 \\
$18131387-3630130$ & -0.07 & +0.10 & +0.08 & -0.04 & 0.05 & 0.16 \\
$18123592-3634476$ & -0.09 & +0.11 & +0.09 & -0.03 & 0.05 & 0.18 \\
$18135736-3638266$ & -0.08 & +0.08 & +0.08 & -0.04 & 0.05 & 0.15 \\
$18133233-3626133$ & -0.09 & +0.11 & +0.09 & -0.03 & 0.07 & 0.18 \\
$18125003-3630269$ & -0.07 & +0.05 & +0.08 & -0.05 & 0.05 & 0.14
\end{tabular}


TABLE 5C-Continued

\begin{tabular}{ccccccc}
\hline \hline Star ID & $\Delta \mathrm{T}_{\text {eff }}$ & $\Delta \log (\mathrm{g})$ & $\Delta[\mathrm{M} / \mathrm{H}]$ & $\Delta \mathrm{V}_{\mathrm{t}}$ & $\sigma / \sqrt{ }(\mathrm{N})$ & $\Delta$ Total \\
2MASS & $+100 \mathrm{~K}$ & $+0.3 \mathrm{cgs}$ & $+0.3 \mathrm{dex}$ & $+0.3 \mathrm{~km} \mathrm{~s}$ & & \\
\hline $18130723-3618385$ & -0.05 & +0.06 & +0.07 & -0.05 & 0.11 & 0.16 \\
$18125800-3616427$ & -0.03 & +0.07 & +0.06 & -0.03 & 0.05 & 0.11 \\
$18111323-3637030$ & -0.05 & +0.07 & +0.06 & -0.04 & 0.15 & 0.19 \\
$18130427-3630012$ & -0.04 & +0.08 & +0.06 & -0.05 & 0.05 & 0.13 \\
$18124614-3617326$ & -0.09 & +0.08 & +0.07 & -0.05 & 0.02 & 0.15 \\
$18120399-3615387$ & -0.08 & +0.09 & +0.07 & -0.03 & 0.09 & 0.17 \\
$18123475-3624478$ & -0.06 & +0.08 & +0.07 & -0.04 & 0.05 & 0.14 \\
$18115661-3617533$ & -0.05 & +0.08 & +0.05 & -0.05 & 0.09 & 0.15 \\
$18113299-3623341$ & -0.09 & +0.07 & +0.06 & -0.05 & 0.03 & 0.14 \\
$18111553-3628156$ & -0.09 & +0.09 & +0.05 & -0.04 & 0.03 & 0.15 \\
$18125907-3644275$ & -0.04 & +0.08 & +0.05 & -0.04 & 0.05 & 0.12 \\
$18111726-3620302$ & $\ldots$ & $\ldots$ & $\ldots$ & $\ldots$ & $\ldots$ & $\ldots$ \\
$18111579-3625114$ & -0.10 & +0.11 & +0.03 & -0.04 & 0.00 & 0.16 \\
$18131305-3650449$ & -0.06 & +0.09 & +0.04 & -0.05 & 0.06 & 0.14 \\
$18123152-3636498$ & -0.11 & +0.09 & +0.03 & -0.05 & 0.08 & 0.17 \\
$18125914-3619208$ & -0.06 & +0.09 & +0.04 & -0.05 & 0.01 & 0.13 \\
$18115073-3650493$ & -0.08 & +0.07 & +0.03 & -0.05 & 0.04 & 0.13 \\
$18114648-3625169$ & -0.04 & +0.07 & +0.04 & -0.06 & 0.09 & 0.14 \\
$18113751-3629139$ & -0.11 & +0.09 & +0.02 & -0.05 & 0.02 & 0.15 \\
$18122243-3637539$ & -0.08 & +0.08 & +0.04 & -0.04 & 0.07 & 0.14 \\
$18133366-3644575$ & -0.04 & +0.08 & +0.05 & -0.05 & 0.03 & 0.12 \\
$18134702-3641477$ & -0.05 & +0.08 & +0.05 & -0.06 & 0.03 & 0.13 \\
$18125228-3618294$ & -0.05 & +0.09 & +0.05 & -0.05 & 0.05 & 0.13 \\
$18110942-3647198$ & -0.04 & +0.09 & +0.05 & -0.04 & 0.05 & 0.13
\end{tabular}


TABLE 5C-Continued

\begin{tabular}{ccccccc}
\hline \hline Star ID & $\Delta \mathrm{T}_{\text {eff }}$ & $\Delta \log (\mathrm{g})$ & $\Delta[\mathrm{M} / \mathrm{H}]$ & $\Delta \mathrm{V}_{\mathrm{t}}$ & $\sigma / \sqrt{ }(\mathrm{N})$ & $\Delta$ Total \\
2MASS & $+100 \mathrm{~K}$ & $+0.3 \mathrm{cgs}$ & $+0.3 \mathrm{dex}$ & $+0.3 \mathrm{~km} \mathrm{~s}$ & & \\
\hline $18104824-3638507$ & -0.10 & +0.10 & +0.03 & -0.04 & 0.05 & 0.16 \\
$18122301-3629462$ & -0.10 & +0.10 & +0.03 & -0.04 & 0.02 & 0.15 \\
$18133332-3645578$ & -0.11 & +0.10 & +0.03 & -0.05 & 0.13 & 0.21 \\
$18123434-3652241$ & -0.08 & +0.07 & +0.04 & -0.05 & 0.01 & 0.12 \\
$18112311-3625456$ & -0.06 & +0.10 & +0.05 & -0.04 & 0.05 & 0.14 \\
$18125463-3649277$ & -0.08 & +0.07 & +0.05 & -0.04 & 0.05 & 0.13 \\
$18123925-3620465$ & -0.10 & +0.08 & +0.04 & -0.04 & 0.02 & 0.14 \\
$18110629-3632146$ & -0.09 & +0.05 & +0.04 & -0.04 & 0.02 & 0.12 \\
$18133687-3639590$ & -0.05 & +0.10 & +0.05 & -0.05 & 0.04 & 0.14 \\
$18111505-3620576$ & -0.05 & +0.10 & +0.05 & -0.05 & 0.03 & 0.14 \\
$18120832-3628243$ & -0.09 & +0.06 & +0.05 & -0.06 & 0.00 & 0.13 \\
$18130815-3631460$ & -0.11 & +0.12 & +0.04 & -0.05 & 0.13 & 0.22 \\
$18120407-3636481$ & -0.09 & +0.08 & +0.04 & -0.04 & 0.02 & 0.13 \\
$18135786-3640300$ & -0.08 & +0.11 & +0.05 & -0.05 & 0.05 & 0.16 \\
$18131228-3619138$ & -0.08 & +0.08 & +0.06 & -0.05 & 0.12 & 0.18 \\
$18113372-3650200$ & $\ldots$ & $\ldots$ & $\ldots$ & $\ldots$ & $\ldots$ & $\ldots$ \\
$18115904-3650428$ & -0.10 & +0.07 & +0.07 & -0.05 & 0.06 & 0.16 \\
$18115448-3641161$ & -0.07 & +0.09 & +0.07 & -0.05 & 0.14 & 0.20 \\
$18123678-3649019$ & -0.05 & +0.09 & +0.08 & -0.04 & 0.10 & 0.17 \\
$18113965-3645408$ & -0.08 & +0.09 & +0.07 & -0.03 & 0.20 & 0.25 \\
$18135362-3636538$ & -0.06 & +0.12 & +0.07 & -0.04 & 0.17 & 0.23 \\
$18123168-3631555$ & -0.04 & +0.09 & +0.07 & -0.04 & 0.05 & 0.14 \\
$18115398-3623290$ & -0.09 & +0.07 & +0.07 & -0.03 & 0.05 & 0.15 \\
$18123393-3615508$ & -0.05 & +0.09 & +0.07 & -0.05 & 0.04 & 0.14
\end{tabular}


TABle 5C-Continued

\begin{tabular}{|c|c|c|c|c|c|c|}
\hline $\begin{array}{l}\text { Star ID } \\
\text { 2MASS }\end{array}$ & $\begin{array}{c}\Delta \mathrm{T}_{\mathrm{eff}} \\
+100 \mathrm{~K}\end{array}$ & $\begin{array}{l}\Delta \log (\mathrm{g}) \\
+0.3 \mathrm{cgs}\end{array}$ & $\begin{array}{l}\Delta[\mathrm{M} / \mathrm{H}] \\
+0.3 \mathrm{dex}\end{array}$ & $\begin{array}{c}\Delta \mathrm{V}_{\mathrm{t}} \\
+0.3 \mathrm{~km} \mathrm{~s}^{-1}\end{array}$ & $\sigma / \sqrt{ }(\mathrm{N})$ & $\Delta$ Total \\
\hline $18120689-3643231$ & -0.05 & +0.11 & +0.07 & -0.05 & 0.10 & 0.18 \\
\hline $18130482-3649019$ & -0.10 & +0.06 & +0.06 & -0.03 & 0.20 & 0.24 \\
\hline $18110352-3646275$ & -0.10 & +0.10 & +0.06 & -0.05 & 0.08 & 0.18 \\
\hline $18105013-3629557$ & -0.08 & +0.10 & +0.06 & -0.05 & 0.10 & 0.18 \\
\hline $18121897-3630243$ & -0.05 & +0.09 & +0.06 & -0.07 & 0.00 & 0.14 \\
\hline $18122029-3652333$ & -0.07 & +0.11 & +0.06 & -0.05 & 0.13 & 0.20 \\
\hline \multicolumn{7}{|c|}{$(\mathrm{l}, \mathrm{b})=(+8.5,+9)$} \\
\hline $17311256-1702018$ & -0.04 & +0.07 & +0.05 & -0.02 & 0.00 & 0.10 \\
\hline $17290874-1632431$ & -0.07 & +0.09 & +0.05 & -0.02 & 0.05 & 0.14 \\
\hline $17302885-1639588$ & -0.06 & +0.06 & +0.06 & -0.02 & 0.05 & 0.12 \\
\hline 17304789-1623192 & -0.05 & +0.05 & +0.07 & -0.02 & 0.02 & 0.10 \\
\hline $17321080-1641479$ & -0.08 & +0.09 & +0.08 & -0.02 & 0.05 & 0.15 \\
\hline $17303589-1638299$ & -0.07 & +0.06 & +0.08 & -0.03 & 0.05 & 0.14 \\
\hline $17290513-1630383$ & -0.09 & +0.08 & +0.08 & -0.03 & 0.01 & 0.15 \\
\hline $17314699-1654460$ & -0.06 & +0.08 & +0.07 & -0.02 & 0.01 & 0.12 \\
\hline $17305076-1615216$ & -0.08 & +0.07 & +0.05 & -0.04 & 0.05 & 0.13 \\
\hline $17323324-1627474$ & -0.11 & +0.11 & +0.03 & -0.04 & 0.10 & 0.19 \\
\hline $17312795-1631423$ & -0.07 & +0.10 & +0.05 & -0.03 & 0.03 & 0.14 \\
\hline $17290285-1638344$ & -0.07 & +0.12 & +0.05 & -0.03 & 0.02 & 0.15 \\
\hline $17303378-1703086$ & -0.10 & +0.11 & +0.05 & -0.04 & 0.02 & 0.16 \\
\hline $17304223-1609274$ & -0.10 & +0.09 & +0.07 & -0.04 & 0.05 & 0.16 \\
\hline 17304908-1633101 & -0.10 & +0.09 & +0.06 & -0.03 & 0.02 & 0.15 \\
\hline $17291459-1650085$ & -0.10 & +0.08 & +0.07 & -0.03 & 0.05 & 0.16 \\
\hline $17300674-1638541$ & -0.11 & +0.08 & +0.07 & -0.04 & 0.06 & 0.17 \\
\hline
\end{tabular}


TABLE 5C-Continued

\begin{tabular}{ccccccc}
\hline \hline Star ID & $\Delta \mathrm{T}_{\text {eff }}$ & $\Delta \log (\mathrm{g})$ & $\Delta[\mathrm{M} / \mathrm{H}]$ & $\Delta \mathrm{V}_{\mathrm{t}}$ & $\sigma / \sqrt{ }(\mathrm{N})$ & $\Delta$ Total \\
2MASS & $+100 \mathrm{~K}$ & $+0.3 \mathrm{cgs}$ & $+0.3 \mathrm{dex}$ & $+0.3 \mathrm{~km} \mathrm{~s}$ & & \\
\hline $17315010-1653413$ & -0.08 & +0.08 & +0.08 & -0.04 & 0.24 & 0.28 \\
$17295493-1613273$ & -0.11 & +0.06 & +0.08 & -0.06 & 0.05 & 0.17 \\
$17311523-1618542$ & -0.08 & +0.08 & +0.09 & -0.05 & 0.09 & 0.18 \\
$17314811-1621558$ & -0.09 & +0.11 & +0.08 & -0.04 & 0.09 & 0.19 \\
$17304861-1646459$ & -0.11 & +0.07 & +0.09 & -0.03 & 0.05 & 0.17 \\
$17323959-1625298$ & -0.09 & +0.10 & +0.09 & -0.05 & 0.03 & 0.17 \\
$17285711-1633566$ & -0.09 & +0.12 & +0.09 & -0.04 & 0.05 & 0.19 \\
$17314673-1641228$ & -0.10 & +0.07 & +0.08 & -0.03 & 0.00 & 0.15 \\
$17294476-1626118$ & -0.11 & +0.08 & +0.07 & -0.03 & 0.04 & 0.16 \\
$17320650-1643389$ & -0.11 & +0.07 & +0.04 & -0.03 & 0.02 & 0.14 \\
$17314427-1640265$ & -0.09 & +0.07 & +0.06 & -0.04 & 0.01 & 0.14 \\
$17300064-1701075$ & -0.11 & +0.07 & +0.04 & -0.03 & 0.05 & 0.15 \\
$17302935-1652277$ & -0.09 & +0.09 & +0.04 & -0.04 & 0.18 & 0.23 \\
$17320652-1619563$ & -0.11 & +0.14 & +0.01 & -0.05 & 0.05 & 0.19 \\
$17304464-1631471$ & -0.10 & +0.10 & +0.03 & -0.04 & 0.02 & 0.15 \\
$17321715-1632467$ & -0.10 & +0.11 & +0.02 & -0.05 & 0.13 & 0.20 \\
$17321969-1653156$ & -0.10 & +0.13 & +0.02 & -0.03 & 0.02 & 0.17 \\
$17315690-1630315$ & -0.11 & +0.12 & +0.02 & -0.05 & 0.05 & 0.18 \\
$17312870-1619572$ & -0.11 & +0.12 & +0.02 & -0.04 & 0.20 & 0.26 \\
$17294541-1649018$ & -0.11 & +0.12 & +0.02 & -0.03 & 0.13 & 0.21 \\
$17305067-1644006$ & -0.11 & +0.13 & +0.02 & -0.03 & 0.11 & 0.21 \\
$17312457-1652524$ & -0.10 & +0.08 & +0.04 & -0.05 & 0.04 & 0.15 \\
$17292760-1636235$ & -0.09 & +0.11 & +0.04 & -0.02 & 0.16 & 0.22 \\
$17295978-1633590$ & -0.11 & +0.15 & +0.02 & -0.04 & 0.08 & 0.21
\end{tabular}


TABLE 5C-Continued

\begin{tabular}{ccccccc}
\hline \hline Star ID & $\Delta \mathrm{T}_{\text {eff }}$ & $\Delta \log (\mathrm{g})$ & $\Delta[\mathrm{M} / \mathrm{H}]$ & $\Delta \mathrm{V}_{\mathrm{t}}$ & $\sigma / \sqrt{ }(\mathrm{N})$ & $\Delta$ Total \\
2MASS & $+100 \mathrm{~K}$ & $+0.3 \mathrm{cgs}$ & $+0.3 \mathrm{dex}$ & $+0.3 \mathrm{~km} \mathrm{~s}$ & & \\
\hline $17322323-1649335$ & -0.10 & +0.08 & +0.03 & -0.04 & 0.00 & 0.14 \\
$17321117-1654590$ & -0.10 & +0.08 & +0.03 & -0.04 & 0.04 & 0.14 \\
$17313606-1607539$ & -0.09 & +0.08 & +0.04 & -0.05 & 0.02 & 0.14 \\
$17295331-1653069$ & -0.10 & +0.08 & +0.04 & -0.04 & 0.05 & 0.15 \\
$17302818-1636032$ & -0.09 & +0.10 & +0.05 & -0.04 & 0.04 & 0.15 \\
$17293118-1638081$ & -0.09 & +0.06 & +0.05 & -0.04 & 0.28 & 0.31 \\
$17291994-1618302$ & -0.11 & +0.14 & +0.04 & -0.03 & 0.09 & 0.21 \\
$17320457-1646391$ & -0.10 & +0.10 & +0.05 & -0.04 & 0.06 & 0.17 \\
$17310747-1620049$ & -0.09 & +0.08 & +0.06 & -0.04 & 0.11 & 0.18 \\
$17323392-1636330$ & $\ldots$ & $\ldots$ & $\ldots$ & $\ldots$ & $\ldots$ & $\ldots$ \\
$17312572-1609092$ & -0.09 & +0.07 & +0.06 & -0.05 & 0.02 & 0.14 \\
$17300015-1609464$ & -0.09 & +0.08 & +0.07 & -0.04 & 0.05 & 0.15 \\
$17305894-1637056$ & -0.10 & +0.16 & +0.06 & -0.03 & 0.05 & 0.21 \\
$17291621-1647189$ & -0.10 & +0.16 & +0.06 & -0.04 & 0.05 & 0.21 \\
$17302194-1643531$ & -0.09 & +0.14 & +0.06 & -0.02 & 0.15 & 0.23 \\
$17311959-1643577$ & -0.09 & +0.09 & +0.06 & -0.03 & 0.00 & 0.14 \\
$17311229-1659047$ & -0.10 & +0.13 & +0.02 & -0.03 & 0.05 & 0.18 \\
$17313313-1659216$ & -0.09 & +0.10 & +0.04 & -0.04 & 0.12 & 0.19 \\
$17301749-1629529$ & -0.10 & +0.06 & +0.05 & -0.04 & 0.13 & 0.19 \\
$17294261-1622440$ & -0.10 & +0.09 & +0.04 & -0.03 & 0.05 & 0.15 \\
$17323625-1637551$ & -0.09 & +0.09 & +0.06 & -0.04 & 0.04 & 0.15 \\
$17314292-1620036$ & -0.10 & +0.11 & +0.06 & -0.03 & 0.01 & 0.16 \\
$17310955-1635152$ & -0.11 & +0.13 & +0.07 & -0.05 & 0.05 & 0.20 \\
$17304312-1630318$ & -0.09 & +0.08 & +0.06 & -0.04 & 0.05 & 0.15
\end{tabular}


TABle 5C-Continued

\begin{tabular}{ccccccc}
\hline \hline $\begin{array}{c}\text { Star ID } \\
\text { 2MASS }\end{array}$ & $\begin{array}{c}\Delta \mathrm{T}_{\text {eff }} \\
+100 \mathrm{~K}\end{array}$ & $\begin{array}{c}\Delta \log (\mathrm{g}) \\
+0.3 \mathrm{cgs}\end{array}$ & $\begin{array}{c}\Delta[\mathrm{M} / \mathrm{H}] \\
+0.3 \mathrm{dex}\end{array}$ & $\begin{array}{c}\Delta \mathrm{V}_{\mathrm{t}} \\
+0.3 \mathrm{~km} \mathrm{~s}{ }^{-1}\end{array}$ & $\sigma / \sqrt{ }(\mathrm{N})$ & $\Delta$ Total \\
\hline $17285469-1627232$ & -0.09 & +0.13 & +0.06 & -0.04 & 0.10 & 0.20 \\
$17310360-1700146$ & -0.10 & +0.07 & +0.06 & -0.05 & 0.05 & 0.15 \\
$17295898-1652101$ & -0.09 & +0.10 & +0.06 & -0.04 & 0.05 & 0.16 \\
\hline
\end{tabular}


TABLE 5D

Abundance UnCertainty: Calcium

\begin{tabular}{ccccccc}
\hline \hline Star ID & $\begin{array}{c}\Delta \mathrm{T}_{\text {eff }} \\
\text { 2MASS }\end{array}$ & $\begin{array}{c}\Delta \log (\mathrm{g}) \\
+100 \mathrm{~K}\end{array}$ & $\begin{array}{c}\Delta[\mathrm{M} / \mathrm{H}] \\
+0.3 \mathrm{cgs}\end{array}$ & $\begin{array}{c}\Delta \mathrm{V}_{\mathrm{t}} \\
+0.3 \mathrm{~km} \mathrm{~s}\end{array}$ & $\sigma / \sqrt{ }(\mathrm{N})$ & $\Delta$ Total \\
\hline \multicolumn{7}{c}{$(\mathrm{l}, \mathrm{b})=(-5.5,-7)$} \\
\hline $18011989-3723462$ & +0.09 & -0.02 & -0.03 & -0.07 & 0.06 & 0.13 \\
$18003452-3729217$ & +0.09 & -0.01 & -0.02 & -0.05 & 0.10 & 0.15 \\
$18011692-3722508$ & +0.11 & -0.01 & -0.02 & -0.09 & 0.06 & 0.16 \\
$18005584-3739103$ & $\ldots$ & $\ldots$ & $\ldots$ & $\ldots$ & $\ldots$ & $\ldots$ \\
$18015010-3735279$ & +0.10 & -0.01 & -0.02 & -0.11 & 0.04 & 0.16 \\
$18005916-3727585$ & +0.11 & -0.02 & -0.02 & -0.12 & 0.03 & 0.17 \\
$18005027-3707505$ & +0.09 & -0.01 & -0.02 & -0.07 & 0.03 & 0.12 \\
$18005234-3715478$ & +0.10 & -0.01 & -0.02 & -0.14 & 0.06 & 0.18 \\
$18020106-3737561$ & +0.11 & -0.01 & -0.02 & -0.14 & 0.05 & 0.19 \\
$17595541-3713447$ & $\ldots$ & $\ldots$ & $\ldots$ & $\ldots$ & $\ldots$ & $\ldots$ \\
$17595664-3725304$ & +0.09 & -0.02 & -0.02 & -0.12 & 0.00 & 0.15 \\
$18021345-3730371$ & +0.12 & -0.01 & -0.02 & -0.15 & 0.04 & 0.20 \\
$17594241-3718247$ & $\ldots$ & $\ldots$ & $\ldots$ & $\ldots$ & $\ldots$ & $\ldots$ \\
$18013342-3726339$ & +0.10 & -0.02 & -0.02 & -0.12 & 0.04 & 0.16 \\
$18003204-3726008$ & +0.11 & -0.02 & +0.00 & -0.17 & 0.07 & 0.22 \\
$18013580-3740055$ & +0.12 & -0.01 & -0.01 & -0.15 & 0.06 & 0.20 \\
$18013799-3725131$ & +0.12 & -0.02 & +0.00 & -0.16 & 0.04 & 0.20 \\
$18022149-3713494$ & +0.11 & -0.01 & -0.01 & -0.14 & 0.02 & 0.18 \\
$18011408-3711299$ & +0.09 & -0.01 & -0.02 & -0.13 & 0.07 & 0.17 \\
$18021275-3737543$ & +0.10 & -0.02 & -0.01 & -0.15 & 0.01 & 0.18 \\
$17595027-3727293$ & +0.11 & -0.02 & -0.01 & -0.15 & 0.06 & 0.20 \\
$18002115-3715519$ & +0.11 & -0.02 & +0.02 & -0.17 & 0.07 & 0.22 \\
$17595366-3728186$ & +0.09 & -0.01 & -0.02 & -0.14 & 0.02 & 0.17
\end{tabular}


TABLE 5D-Continued

\begin{tabular}{ccccccc}
\hline \hline Star ID & $\Delta \mathrm{T}_{\text {eff }}$ & $\Delta \log (\mathrm{g})$ & $\Delta[\mathrm{M} / \mathrm{H}]$ & $\Delta \mathrm{V}_{\mathrm{t}}$ & $\sigma / \sqrt{ }(\mathrm{N})$ & $\Delta$ Total \\
2MASS & $+100 \mathrm{~K}$ & $+0.3 \mathrm{cgs}$ & $+0.3 \mathrm{dex}$ & $+0.3 \mathrm{~km} \mathrm{~s}$ & & \\
\hline $18001354-3709122$ & +0.11 & -0.01 & +0.02 & -0.17 & 0.02 & 0.20 \\
$18013960-3723398$ & +0.10 & -0.02 & +0.02 & -0.17 & 0.03 & 0.20 \\
$18021862-3734204$ & +0.11 & -0.01 & +0.01 & -0.16 & 0.05 & 0.20 \\
$18000535-3735315$ & +0.11 & -0.01 & +0.01 & -0.17 & 0.01 & 0.20 \\
$18013848-3721442$ & +0.11 & -0.01 & +0.01 & -0.16 & 0.05 & 0.20 \\
$17594792-3721422$ & +0.11 & -0.03 & +0.00 & -0.16 & 0.02 & 0.20 \\
$18011941-3721250$ & +0.11 & +0.00 & +0.02 & -0.16 & 0.02 & 0.20 \\
$18004824-3718074$ & +0.10 & -0.02 & +0.03 & -0.18 & 0.03 & 0.21 \\
$18014343-3708232$ & +0.11 & -0.01 & +0.02 & -0.17 & 0.11 & 0.23 \\
$18005852-3711524$ & +0.09 & -0.03 & -0.01 & -0.16 & 0.08 & 0.20 \\
$18020816-3714015$ & +0.10 & -0.01 & -0.01 & -0.15 & 0.05 & 0.19 \\
$18010932-3702079$ & +0.10 & -0.01 & +0.03 & -0.17 & 0.03 & 0.20 \\
$18010065-3740456$ & +0.10 & -0.01 & +0.03 & -0.18 & 0.05 & 0.21 \\
$17595640-3710383$ & +0.11 & -0.03 & +0.00 & -0.17 & 0.00 & 0.20 \\
$18012462-3738382$ & +0.10 & -0.01 & -0.01 & -0.15 & 0.06 & 0.19 \\
$18002880-3710171$ & +0.09 & -0.01 & -0.02 & -0.15 & 0.05 & 0.18 \\
$18024484-3714159$ & +0.09 & -0.01 & -0.01 & -0.15 & 0.07 & 0.19 \\
$18013206-3708133$ & $\ldots$ & $\ldots$ & $\ldots$ & $\ldots$ & $\ldots$ & $\ldots$ \\
$18004074-3740058$ & +0.11 & -0.02 & -0.01 & -0.16 & 0.05 & 0.20 \\
$18000013-3715053$ & +0.11 & -0.02 & +0.00 & -0.16 & 0.08 & 0.21 \\
$18015431-3717070$ & +0.10 & -0.01 & -0.01 & -0.16 & 0.05 & 0.20 \\
$18021006-3738193$ & +0.08 & -0.02 & -0.02 & -0.16 & 0.05 & 0.19 \\
$17595442-3721118$ & +0.11 & -0.01 & +0.00 & -0.17 & 0.03 & 0.20 \\
$18021721-3736096$ & +0.09 & +0.00 & -0.02 & -0.14 & 0.09 & 0.19
\end{tabular}


TABLE 5D-Continued

\begin{tabular}{ccccccc}
\hline \hline Star ID & $\begin{array}{c}\Delta \mathrm{T}_{\text {eff }} \\
\text { 2MASS }\end{array}$ & $\begin{array}{c}\Delta \log (\mathrm{g}) \\
+0.3 \mathrm{cgs}\end{array}$ & $\begin{array}{c}\Delta[\mathrm{M} / \mathrm{H}] \\
+0.3 \mathrm{dex}\end{array}$ & $\begin{array}{c}\Delta \mathrm{V}_{\mathrm{t}} \\
+0.3 \mathrm{~km} \mathrm{~s}\end{array}$ & $\sigma / \sqrt{ }(\mathrm{N})$ & $\Delta$ Total \\
\hline $18005096-3733499$ & $\ldots$ & $\ldots$ & $\ldots$ & $\ldots$ & $\ldots$ & $\ldots$ \\
$18005923-3707111$ & +0.09 & -0.02 & +0.01 & -0.18 & 0.02 & 0.20 \\
$18011851-3706092$ & +0.10 & -0.02 & +0.01 & -0.18 & 0.05 & 0.21 \\
$18013395-3705551$ & +0.09 & -0.03 & +0.03 & -0.18 & 0.08 & 0.22 \\
$18022648-3728188$ & +0.09 & +0.00 & -0.02 & -0.15 & 0.04 & 0.18 \\
$18024919-3727234$ & +0.09 & +0.00 & -0.01 & -0.15 & 0.06 & 0.19 \\
$18023660-3716289$ & +0.09 & +0.00 & -0.01 & -0.14 & 0.08 & 0.18 \\
$17595509-3711099$ & +0.10 & -0.01 & +0.00 & -0.17 & 0.05 & 0.20 \\
$18005395-3731231$ & +0.08 & -0.01 & +0.02 & -0.18 & 0.09 & 0.22 \\
$18010322-3705447$ & +0.08 & +0.00 & -0.01 & -0.15 & 0.03 & 0.17 \\
$18014769-3739220$ & $\ldots$ & $\ldots$ & $\ldots$ & $\ldots$ & $\ldots$ & $\ldots$ \\
$17594807-3715283$ & +0.09 & -0.02 & +0.00 & -0.17 & 0.04 & 0.20 \\
$18013618-3706311$ & +0.10 & -0.02 & +0.02 & -0.18 & 0.03 & 0.21 \\
$18004314-3730156$ & +0.09 & -0.01 & +0.00 & -0.17 & 0.03 & 0.19 \\
$18014303-3740054$ & +0.10 & -0.02 & +0.02 & -0.18 & 0.06 & 0.22 \\
$18003751-3728200$ & +0.08 & -0.01 & +0.04 & -0.20 & 0.07 & 0.23 \\
$18011170-3727118$ & +0.09 & +0.00 & +0.00 & -0.12 & 0.02 & 0.15 \\
$18023846-3718345$ & +0.10 & -0.02 & +0.03 & -0.18 & 0.05 & 0.21 \\
$18011671-3722213$ & $\ldots$ & $\ldots$ & $\ldots$ & $\ldots$ & $\ldots$ & $\ldots$ \\
$18005985-3734507$ & $\ldots$ & $\ldots$ & $\ldots$ & $\ldots$ & $\ldots$ & $\ldots$ \\
$18000430-3711051$ & +0.10 & -0.02 & +0.04 & -0.19 & 0.01 & 0.22 \\
$18003552-3729476$ & +0.10 & -0.01 & +0.03 & -0.18 & 0.07 & 0.22 \\
$18001817-3732243$ & +0.09 & -0.02 & +0.02 & -0.19 & 0.09 & 0.23 \\
$18013039-3713086$ & +0.09 & -0.01 & +0.03 & -0.17 & 0.01 & 0.20
\end{tabular}


TABLE 5D-Continued

\begin{tabular}{ccccccc}
\hline \hline Star ID & $\begin{array}{c}\Delta \mathrm{T}_{\text {eff }} \\
\text { 2MASS }\end{array}$ & $\begin{array}{c}\Delta \log (\mathrm{g}) \\
+0.3 \mathrm{cgs}\end{array}$ & $\begin{array}{c}\Delta[\mathrm{M} / \mathrm{H}] \\
+0.3 \mathrm{dex}\end{array}$ & $\begin{array}{c}\Delta \mathrm{V}_{\mathrm{t}} \\
+0.3 \mathrm{~km} \mathrm{~s}\end{array}$ & $\sigma / \sqrt{ }(\mathrm{N})$ & $\Delta$ Total \\
\hline $18012564-3741052$ & +0.10 & -0.01 & +0.03 & -0.18 & 0.05 & 0.21 \\
$18025713-3718318$ & +0.08 & -0.01 & +0.04 & -0.20 & 0.09 & 0.24 \\
$18011599-3741385$ & +0.09 & -0.02 & +0.03 & -0.19 & 0.05 & 0.22 \\
$18012944-3719488$ & +0.09 & +0.00 & +0.00 & -0.15 & 0.06 & 0.18 \\
$18020361-3719086$ & +0.09 & +0.00 & +0.01 & -0.17 & 0.10 & 0.22 \\
$18024024-3728187$ & +0.08 & -0.01 & +0.04 & -0.18 & 0.10 & 0.22 \\
$18000412-3717266$ & +0.07 & -0.02 & +0.05 & -0.19 & 0.03 & 0.21 \\
$18011177-3703344$ & +0.09 & -0.02 & +0.05 & -0.19 & 0.09 & 0.23 \\
$18001410-3725181$ & +0.07 & -0.02 & +0.06 & -0.20 & 0.06 & 0.23 \\
$18020300-3719418$ & +0.07 & -0.03 & +0.05 & -0.20 & 0.05 & 0.23 \\
$18004683-3703252$ & +0.07 & -0.02 & +0.05 & -0.19 & 0.04 & 0.21 \\
$18015404-3722564$ & +0.10 & -0.02 & +0.03 & -0.19 & 0.03 & 0.22 \\
$18013974-3704068$ & +0.09 & -0.01 & +0.03 & -0.19 & 0.04 & 0.22 \\
$18020704-3708064$ & +0.09 & +0.00 & +0.00 & -0.19 & 0.00 & 0.21 \\
$18023393-3730273$ & $\ldots$ & $\ldots$ & $\ldots$ & $\ldots$ & $\ldots$ & $\ldots$ \\
$18001720-3708374$ & +0.06 & -0.02 & +0.06 & -0.20 & 0.04 & 0.22 \\
$18003663-3708547$ & +0.08 & -0.02 & +0.04 & -0.20 & 0.04 & 0.22 \\
$18002569-3729578$ & +0.09 & -0.01 & +0.02 & -0.20 & 0.09 & 0.24 \\
$17594881-3716203$ & +0.09 & -0.03 & +0.06 & -0.19 & 0.03 & 0.22 \\
$18011201-3740090$ & +0.09 & +0.00 & +0.02 & -0.19 & 0.05 & 0.22 \\
\hline & & & $(1, \mathrm{~b})=(-4,-9)$ & & & \\
\hline $18122552-3653049$ & +0.10 & -0.02 & -0.02 & -0.08 & 0.05 & 0.14 \\
$18120653-3654264$ & +0.08 & -0.02 & -0.02 & -0.08 & 0.04 & 0.12 \\
$18110179-3630394$ & +0.10 & -0.02 & -0.02 & -0.08 & 0.05 & 0.14
\end{tabular}


TABLE 5D-Continued

\begin{tabular}{ccccccc}
\hline \hline Star ID & $\Delta \mathrm{T}_{\text {eff }}$ & $\Delta \log (\mathrm{g})$ & $\Delta[\mathrm{M} / \mathrm{H}]$ & $\Delta \mathrm{V}_{\mathrm{t}}$ & $\sigma / \sqrt{ }(\mathrm{N})$ & $\Delta$ Total \\
2MASS & $+100 \mathrm{~K}$ & $+0.3 \mathrm{cgs}$ & $+0.3 \mathrm{dex}$ & $+0.3 \mathrm{~km} \mathrm{~s}$ & & \\
\hline $18124892-3626403$ & +0.08 & -0.02 & -0.02 & -0.08 & 0.03 & 0.12 \\
$18135164-3643463$ & +0.10 & -0.02 & -0.02 & -0.07 & 0.06 & 0.14 \\
$18124339-3635339$ & +0.11 & -0.02 & -0.02 & -0.10 & 0.11 & 0.19 \\
$18113921-3652069$ & +0.10 & -0.01 & -0.02 & -0.08 & 0.05 & 0.14 \\
$18111325-3646007$ & +0.11 & -0.02 & -0.02 & -0.12 & 0.00 & 0.17 \\
$18113833-3626322$ & +0.12 & -0.01 & -0.02 & -0.12 & 0.06 & 0.18 \\
$18111018-3639350$ & +0.11 & -0.02 & -0.02 & -0.12 & 0.06 & 0.18 \\
$18135243-3630481$ & +0.10 & -0.02 & -0.02 & -0.09 & 0.06 & 0.15 \\
$18121150-3627559$ & +0.10 & -0.01 & -0.02 & -0.10 & 0.04 & 0.15 \\
$18111963-3647245$ & +0.11 & -0.01 & -0.02 & -0.14 & 0.02 & 0.18 \\
$18140023-3635461$ & +0.10 & +0.00 & -0.02 & -0.11 & 0.05 & 0.16 \\
$18135881-3639345$ & +0.11 & -0.01 & -0.02 & -0.13 & 0.05 & 0.18 \\
$18121436-3654551$ & +0.12 & -0.01 & -0.02 & -0.14 & 0.04 & 0.19 \\
$18124228-3655079$ & +0.12 & -0.01 & -0.01 & -0.13 & 0.04 & 0.18 \\
$18122530-3637366$ & +0.12 & -0.01 & -0.02 & -0.14 & 0.00 & 0.19 \\
$18131370-3651584$ & +0.10 & -0.01 & -0.02 & -0.15 & 0.04 & 0.19 \\
$18134668-3623541$ & +0.11 & -0.01 & -0.02 & -0.12 & 0.03 & 0.17 \\
$18105081-3642137$ & +0.09 & -0.03 & -0.02 & -0.15 & 0.00 & 0.18 \\
$18124219-3631172$ & +0.11 & -0.01 & -0.02 & -0.14 & 0.03 & 0.18 \\
$18133688-3647054$ & $\ldots$ & $\ldots$ & $\ldots$ & $\ldots$ & $\ldots$ & $\ldots$ \\
$18123142-3639294$ & +0.12 & -0.01 & -0.01 & -0.16 & 0.01 & 0.20 \\
$18133038-3632229$ & $\ldots$ & $\ldots$ & $\ldots$ & $\ldots$ & & $\ldots$ \\
$18133292-3624179$ & $\ldots$ & $\ldots$ & $\ldots$ & $\ldots$ & & $\ldots$ \\
$18111681-3636318$ & +0.11 & -0.02 & -0.01 & -0.16 & 0.02 & 0.20
\end{tabular}


TABLE 5D-Continued

\begin{tabular}{ccccccc}
\hline \hline Star ID & $\begin{array}{c}\Delta \mathrm{T}_{\text {eff }} \\
\text { 2MASS }\end{array}$ & $\begin{array}{c}\Delta \log (\mathrm{g}) \\
+0.3 \mathrm{cgs}\end{array}$ & $\begin{array}{c}\Delta[\mathrm{M} / \mathrm{H}] \\
+0.3 \mathrm{dex}\end{array}$ & $\begin{array}{c}\Delta \mathrm{V}_{\mathrm{t}} \\
+0.3 \mathrm{~km} \mathrm{~s}\end{array}$ & $\sigma / \sqrt{ }(\mathrm{N})$ & $\Delta$ Total \\
\hline $18105004-3640063$ & $\ldots$ & $\ldots$ & $\ldots$ & $\ldots$ & & \\
$18112334-3634194$ & +0.10 & -0.03 & -0.02 & -0.15 & 0.05 & 0.19 \\
$18131027-3632343$ & +0.11 & -0.02 & -0.02 & -0.15 & 0.00 & 0.19 \\
$18110293-3628041$ & +0.12 & -0.01 & -0.01 & -0.15 & 0.06 & 0.20 \\
$18132040-3623075$ & +0.11 & -0.03 & -0.01 & -0.15 & 0.05 & 0.20 \\
$18135588-3629188$ & +0.10 & -0.01 & -0.02 & -0.12 & 0.06 & 0.17 \\
$18105075-3635061$ & +0.11 & -0.02 & +0.00 & -0.17 & 0.03 & 0.21 \\
$18104188-3634541$ & +0.09 & +0.00 & -0.02 & -0.11 & 0.06 & 0.16 \\
$18132998-3628578$ & +0.11 & -0.01 & +0.00 & -0.15 & 0.03 & 0.19 \\
$18130544-3616563$ & +0.12 & -0.02 & +0.00 & -0.16 & 0.03 & 0.20 \\
$18115908-3654510$ & +0.11 & -0.01 & +0.00 & -0.17 & 0.04 & 0.21 \\
$18121077-3639094$ & +0.11 & -0.02 & -0.01 & -0.14 & 0.03 & 0.18 \\
$18120614-3629571$ & +0.10 & -0.02 & -0.01 & -0.15 & 0.03 & 0.18 \\
$18120213-3641599$ & +0.09 & -0.01 & -0.02 & -0.14 & 0.01 & 0.17 \\
$18123350-3654398$ & +0.10 & -0.02 & +0.02 & -0.17 & 0.07 & 0.21 \\
$18120973-3654184$ & +0.10 & -0.01 & +0.03 & -0.16 & 0.04 & 0.20 \\
$18105641-3632359$ & +0.11 & -0.03 & +0.00 & -0.16 & 0.04 & 0.20 \\
$18125106-3654091$ & +0.09 & -0.01 & -0.01 & -0.15 & 0.05 & 0.18 \\
$18111581-3648469$ & +0.10 & -0.02 & +0.03 & -0.18 & 0.03 & 0.21 \\
$18131387-3630130$ & +0.12 & -0.01 & +0.01 & -0.16 & 0.01 & 0.20 \\
$18123592-3634476$ & +0.10 & -0.01 & +0.03 & -0.16 & 0.05 & 0.20 \\
$18135736-3638266$ & +0.11 & -0.03 & +0.02 & -0.17 & 0.01 & 0.21 \\
$18133233-3626133$ & +0.10 & -0.01 & +0.03 & -0.17 & 0.05 & 0.21 \\
$18125003-3630269$ & +0.10 & -0.02 & -0.01 & -0.14 & 0.05 & 0.18
\end{tabular}


TABLE 5D-Continued

\begin{tabular}{ccccccc}
\hline \hline Star ID & $\Delta \mathrm{T}_{\text {eff }}$ & $\Delta \log (\mathrm{g})$ & $\Delta[\mathrm{M} / \mathrm{H}]$ & $\Delta \mathrm{V}_{\mathrm{t}}$ & $\sigma / \sqrt{ }(\mathrm{N})$ & $\Delta$ Total \\
2MASS & $+100 \mathrm{~K}$ & $+0.3 \mathrm{cgs}$ & $+0.3 \mathrm{dex}$ & $+0.3 \mathrm{~km} \mathrm{~s}$ & & \\
\hline $18130723-3618385$ & +0.10 & -0.02 & -0.01 & -0.16 & 0.05 & 0.20 \\
$18125800-3616427$ & +0.09 & -0.02 & -0.01 & -0.15 & 0.06 & 0.19 \\
$18111323-3637030$ & +0.09 & -0.02 & -0.01 & -0.16 & 0.07 & 0.20 \\
$18130427-3630012$ & +0.08 & -0.01 & -0.02 & -0.14 & 0.09 & 0.19 \\
$18124614-3617326$ & +0.11 & -0.01 & +0.00 & -0.16 & 0.07 & 0.21 \\
$18120399-3615387$ & +0.11 & -0.02 & +0.02 & -0.17 & 0.08 & 0.22 \\
$18123475-3624478$ & +0.10 & -0.02 & -0.01 & -0.15 & 0.00 & 0.18 \\
$18115661-3617533$ & +0.09 & -0.02 & -0.02 & -0.16 & 0.05 & 0.19 \\
$18113299-3623341$ & +0.11 & -0.03 & +0.01 & -0.18 & 0.05 & 0.22 \\
$18111553-3628156$ & +0.11 & -0.02 & +0.02 & -0.17 & 0.02 & 0.21 \\
$18125907-3644275$ & +0.09 & +0.00 & -0.02 & -0.12 & 0.06 & 0.16 \\
$18111726-3620302$ & +0.11 & -0.01 & -0.01 & -0.15 & 0.02 & 0.19 \\
$18111579-3625114$ & +0.09 & -0.04 & +0.04 & -0.18 & 0.03 & 0.21 \\
$18131305-3650449$ & +0.09 & -0.02 & -0.02 & -0.16 & 0.01 & 0.19 \\
$18123152-3636498$ & +0.09 & -0.03 & +0.03 & -0.18 & 0.05 & 0.21 \\
$18125914-3619208$ & +0.09 & -0.01 & -0.02 & -0.17 & 0.02 & 0.19 \\
$18115073-3650493$ & +0.10 & -0.01 & -0.02 & -0.15 & 0.05 & 0.19 \\
$18114648-3625169$ & +0.08 & -0.01 & -0.02 & -0.15 & 0.06 & 0.18 \\
$18113751-3629139$ & +0.09 & -0.03 & +0.03 & -0.18 & 0.07 & 0.22 \\
$18122243-3637539$ & +0.10 & -0.02 & +0.00 & -0.17 & 0.06 & 0.21 \\
$18133366-3644575$ & $\ldots$ & $\ldots$ & $\ldots$ & $\ldots$ & & $\ldots$ \\
$18134702-3641477$ & +0.08 & -0.01 & -0.02 & -0.16 & 0.03 & 0.18 \\
$18125228-3618294$ & +0.09 & -0.01 & -0.02 & -0.17 & 0.07 & 0.21 \\
$18110942-3647198$ & +0.08 & +0.00 & -0.01 & -0.13 & 0.09 & 0.18
\end{tabular}


TABLE 5D-Continued

\begin{tabular}{ccccccc}
\hline \hline Star ID & $\Delta \mathrm{T}_{\text {eff }}$ & $\Delta \log (\mathrm{g})$ & $\Delta[\mathrm{M} / \mathrm{H}]$ & $\Delta \mathrm{V}_{\mathrm{t}}$ & $\sigma / \sqrt{ }(\mathrm{N})$ & $\Delta$ Total \\
2MASS & $+100 \mathrm{~K}$ & $+0.3 \mathrm{cgs}$ & $+0.3 \mathrm{dex}$ & $+0.3 \mathrm{~km} \mathrm{~s}$ & & \\
\hline $18104824-3638507$ & +0.09 & -0.02 & +0.03 & -0.19 & 0.05 & 0.22 \\
$18122301-3629462$ & +0.08 & -0.01 & +0.03 & -0.18 & 0.03 & 0.20 \\
$18133332-3645578$ & +0.08 & -0.02 & +0.03 & -0.18 & 0.02 & 0.20 \\
$18123434-3652241$ & +0.10 & -0.02 & +0.00 & -0.17 & 0.05 & 0.20 \\
$18112311-3625456$ & +0.09 & -0.01 & -0.01 & -0.16 & 0.03 & 0.19 \\
$18125463-3649277$ & +0.10 & -0.02 & +0.00 & -0.17 & 0.06 & 0.21 \\
$18123925-3620465$ & +0.09 & -0.03 & +0.02 & -0.18 & 0.08 & 0.22 \\
$18110629-3632146$ & +0.10 & -0.02 & +0.01 & -0.17 & 0.04 & 0.20 \\
$18133687-3639590$ & +0.09 & -0.01 & -0.01 & -0.17 & 0.07 & 0.21 \\
$18111505-3620576$ & +0.09 & -0.02 & +0.00 & -0.18 & 0.03 & 0.20 \\
$18120832-3628243$ & +0.10 & -0.03 & +0.02 & -0.18 & 0.01 & 0.21 \\
$18130815-3631460$ & +0.08 & -0.02 & +0.04 & -0.19 & 0.03 & 0.21 \\
$18120407-3636481$ & +0.09 & -0.02 & +0.03 & -0.19 & 0.04 & 0.22 \\
$18135786-3640300$ & +0.10 & -0.01 & +0.02 & -0.20 & 0.01 & 0.22 \\
$18131228-3619138$ & +0.10 & -0.02 & +0.01 & -0.18 & 0.07 & 0.22 \\
$18113372-3650200$ & +0.10 & -0.01 & +0.03 & -0.18 & 0.03 & 0.21 \\
$18115904-3650428$ & +0.08 & -0.02 & +0.04 & -0.19 & 0.01 & 0.21 \\
$18115448-3641161$ & +0.09 & -0.01 & +0.01 & -0.17 & 0.05 & 0.20 \\
$18123678-3649019$ & +0.10 & -0.02 & +0.02 & -0.18 & 0.03 & 0.21 \\
$18113965-3645408$ & +0.10 & -0.02 & +0.03 & -0.18 & 0.06 & 0.22 \\
$18135362-3636538$ & +0.10 & -0.02 & +0.02 & -0.18 & 0.02 & 0.21 \\
$18123168-3631555$ & +0.09 & -0.01 & +0.00 & -0.17 & 0.02 & 0.19 \\
$18115398-3623290$ & +0.07 & -0.02 & +0.06 & -0.19 & 0.03 & 0.21 \\
$18123393-3615508$ & +0.09 & -0.01 & +0.00 & -0.17 & 0.02 & 0.19
\end{tabular}


TABLE 5D-Continued

\begin{tabular}{ccccccc}
\hline \hline Star ID & $\begin{array}{c}\Delta \mathrm{T}_{\text {eff }} \\
\text { 2MASS }\end{array}$ & $\begin{array}{c}\Delta \log (\mathrm{g}) \\
+0.3 \mathrm{cgs}\end{array}$ & $\begin{array}{c}\Delta[\mathrm{M} / \mathrm{H}] \\
+0.3 \mathrm{dex}\end{array}$ & $\begin{array}{c}\Delta \mathrm{V}_{\mathrm{t}} \\
+0.3 \mathrm{~km} \mathrm{~s}\end{array}$ & $\sigma / \sqrt{ }(\mathrm{N})$ & $\Delta$ Total \\
\hline $18120689-3643231$ & +0.09 & +0.00 & +0.00 & -0.17 & 0.07 & 0.20 \\
$18130482-3649019$ & $\ldots$ & $\ldots$ & $\ldots$ & $\ldots$ & & $\ldots$ \\
$18110352-3646275$ & +0.07 & -0.01 & +0.04 & -0.20 & 0.08 & 0.23 \\
$18105013-3629557$ & +0.09 & -0.03 & +0.04 & -0.19 & 0.01 & 0.22 \\
$18121897-3630243$ & +0.09 & -0.02 & +0.01 & -0.19 & 0.05 & 0.22 \\
$18122029-3652333$ & +0.09 & -0.01 & +0.02 & -0.19 & 0.04 & 0.22 \\
\hline & & $(1, \mathrm{~b})=(+8.5,+9)$ & & & \\
\hline $17311256-1702018$ & +0.12 & -0.01 & -0.02 & -0.11 & 0.03 & 0.17 \\
$17290874-1632431$ & +0.12 & -0.01 & -0.02 & -0.11 & 0.01 & 0.16 \\
$17302885-1639588$ & +0.12 & -0.02 & -0.02 & -0.12 & 0.02 & 0.17 \\
$17304789-1623192$ & +0.12 & -0.02 & -0.02 & -0.13 & 0.05 & 0.19 \\
$17321080-1641479$ & +0.13 & -0.01 & +0.00 & -0.14 & 0.02 & 0.19 \\
$17303589-1638299$ & +0.12 & -0.02 & -0.01 & -0.15 & 0.05 & 0.20 \\
$17290513-1630383$ & +0.13 & -0.01 & +0.00 & -0.14 & 0.10 & 0.22 \\
$17314699-1654460$ & +0.12 & -0.01 & -0.01 & -0.14 & 0.00 & 0.18 \\
$17305076-1615216$ & +0.12 & -0.02 & -0.02 & -0.15 & 0.06 & 0.20 \\
$17323324-1627474$ & $\ldots$ & $\ldots$ & $\ldots$ & $\ldots$ & $\ldots$ & $\ldots$ \\
$17312795-1631423$ & +0.12 & +0.00 & -0.02 & -0.13 & 0.02 & 0.18 \\
$17290285-1638344$ & +0.12 & +0.00 & -0.02 & -0.13 & 0.04 & 0.18 \\
$17303378-1703086$ & +0.11 & -0.01 & +0.01 & -0.18 & 0.04 & 0.22 \\
$17304223-1609274$ & +0.11 & -0.01 & +0.01 & -0.16 & 0.01 & 0.19 \\
$17304908-1633101$ & +0.11 & -0.01 & +0.01 & -0.15 & 0.07 & 0.20 \\
$17291459-1650085$ & +0.11 & -0.01 & +0.02 & -0.15 & 0.03 & 0.19 \\
$17300674-1638541$ & +0.10 & -0.02 & +0.03 & -0.18 & 0.05 & 0.21
\end{tabular}


TABLE 5D-Continued

\begin{tabular}{ccccccc}
\hline \hline Star ID & $\Delta \mathrm{T}_{\text {eff }}$ & $\Delta \log (\mathrm{g})$ & $\Delta[\mathrm{M} / \mathrm{H}]$ & $\Delta \mathrm{V}_{\mathrm{t}}$ & $\sigma / \sqrt{ }(\mathrm{N})$ & $\Delta$ Total \\
2MASS & $+100 \mathrm{~K}$ & $+0.3 \mathrm{cgs}$ & $+0.3 \mathrm{dex}$ & $+0.3 \mathrm{~km} \mathrm{~s}$ & & \\
\hline $17315010-1653413$ & +0.12 & -0.02 & +0.01 & -0.16 & 0.08 & 0.22 \\
$17295493-1613273$ & +0.10 & -0.02 & +0.03 & -0.18 & 0.07 & 0.22 \\
$17311523-1618542$ & $\ldots$ & $\ldots$ & $\ldots$ & $\ldots$ & $\ldots$ & $\ldots$ \\
$17314811-1621558$ & +0.10 & +0.00 & +0.02 & -0.15 & 0.01 & 0.18 \\
$17304861-1646459$ & +0.10 & -0.02 & +0.04 & -0.18 & 0.10 & 0.23 \\
$17323959-1625298$ & +0.11 & -0.01 & +0.02 & -0.17 & 0.02 & 0.20 \\
$17285711-1633566$ & +0.10 & -0.01 & +0.03 & -0.17 & 0.02 & 0.20 \\
$17314673-1641228$ & +0.10 & -0.02 & +0.04 & -0.18 & 0.05 & 0.22 \\
$17294476-1626118$ & +0.10 & -0.01 & +0.04 & -0.17 & 0.05 & 0.21 \\
$17320650-1643389$ & +0.10 & -0.01 & +0.03 & -0.19 & 0.06 & 0.23 \\
$17314427-1640265$ & +0.11 & -0.02 & +0.01 & -0.16 & 0.04 & 0.20 \\
$17300064-1701075$ & +0.09 & -0.02 & +0.03 & -0.19 & 0.03 & 0.22 \\
$17302935-1652277$ & +0.10 & -0.01 & +0.00 & -0.15 & 0.00 & 0.18 \\
$17320652-1619563$ & +0.09 & -0.03 & +0.03 & -0.19 & 0.05 & 0.22 \\
$17304464-1631471$ & +0.09 & -0.01 & +0.02 & -0.17 & 0.00 & 0.19 \\
$17321715-1632467$ & +0.09 & +0.00 & +0.01 & -0.17 & 0.01 & 0.19 \\
$17321969-1653156$ & +0.09 & -0.01 & +0.03 & -0.19 & 0.02 & 0.21 \\
$17315690-1630315$ & +0.08 & -0.01 & +0.03 & -0.19 & 0.03 & 0.21 \\
$17312870-1619572$ & +0.09 & -0.01 & +0.04 & -0.19 & 0.03 & 0.22 \\
$17294541-1649018$ & +0.09 & -0.01 & +0.03 & -0.19 & 0.04 & 0.22 \\
$17305067-1644006$ & +0.09 & +0.00 & +0.03 & -0.18 & 0.11 & 0.23 \\
$17312457-1652524$ & +0.09 & -0.02 & +0.02 & -0.18 & 0.06 & 0.21 \\
$17292760-1636235$ & +0.09 & -0.01 & +0.04 & -0.18 & 0.05 & 0.21 \\
$17295978-1633590$ & +0.08 & +0.00 & +0.03 & -0.19 & 0.06 & 0.22
\end{tabular}


TABLE 5D-Continued

\begin{tabular}{ccccccc}
\hline \hline Star ID & $\Delta \mathrm{T}_{\text {eff }}$ & $\Delta \log (\mathrm{g})$ & $\Delta[\mathrm{M} / \mathrm{H}]$ & $\Delta \mathrm{V}_{\mathrm{t}}$ & $\sigma / \sqrt{ }(\mathrm{N})$ & $\Delta$ Total \\
2MASS & $+100 \mathrm{~K}$ & $+0.3 \mathrm{cgs}$ & $+0.3 \mathrm{dex}$ & $+0.3 \mathrm{~km} \mathrm{~s}$ & & \\
\hline $17322323-1649335$ & +0.09 & -0.01 & +0.02 & -0.17 & 0.01 & 0.19 \\
$17321117-1654590$ & +0.09 & -0.02 & +0.03 & -0.18 & 0.02 & 0.21 \\
$17313606-1607539$ & +0.10 & -0.01 & +0.01 & -0.17 & 0.04 & 0.20 \\
$17295331-1653069$ & +0.09 & -0.02 & +0.03 & -0.19 & 0.02 & 0.21 \\
$17302818-1636032$ & +0.08 & -0.02 & +0.03 & -0.19 & 0.05 & 0.22 \\
$17293118-1638081$ & +0.09 & -0.02 & +0.02 & -0.17 & 0.04 & 0.20 \\
$17291994-1618302$ & +0.08 & +0.02 & +0.04 & -0.17 & 0.09 & 0.21 \\
$17320457-1646391$ & +0.08 & -0.01 & +0.04 & -0.19 & 0.07 & 0.22 \\
$17310747-1620049$ & +0.09 & -0.02 & +0.04 & -0.19 & 0.02 & 0.22 \\
$17323392-1636330$ & +0.08 & -0.01 & +0.06 & -0.19 & 0.07 & 0.23 \\
$17312572-1609092$ & +0.10 & -0.02 & +0.03 & -0.19 & 0.03 & 0.22 \\
$17300015-1609464$ & +0.09 & -0.02 & +0.04 & -0.19 & 0.05 & 0.22 \\
$17305894-1637056$ & +0.07 & +0.00 & +0.06 & -0.20 & 0.05 & 0.23 \\
$17291621-1647189$ & +0.07 & +0.01 & +0.06 & -0.20 & 0.05 & 0.23 \\
$17302194-1643531$ & +0.07 & +0.00 & +0.06 & -0.20 & 0.07 & 0.23 \\
$17311959-1643577$ & +0.07 & -0.01 & +0.05 & -0.19 & 0.09 & 0.23 \\
$17311229-1659047$ & +0.07 & +0.00 & +0.05 & -0.20 & 0.08 & 0.23 \\
$17313313-1659216$ & +0.08 & -0.02 & +0.04 & -0.19 & 0.01 & 0.21 \\
$17301749-1629529$ & +0.06 & -0.02 & +0.04 & -0.20 & 0.07 & 0.22 \\
$17294261-1622440$ & +0.06 & -0.01 & +0.04 & -0.20 & 0.05 & 0.22 \\
$17323625-1637551$ & +0.08 & -0.02 & +0.04 & -0.20 & 0.07 & 0.23 \\
$17314292-1620036$ & +0.06 & +0.00 & +0.06 & -0.20 & 0.02 & 0.22 \\
$17310955-1635152$ & +0.06 & +0.00 & +0.06 & -0.21 & 0.08 & 0.24 \\
$17304312-1630318$ & +0.08 & -0.02 & +0.06 & -0.20 & 0.02 & 0.23
\end{tabular}


TABLE 5D-Continued

\begin{tabular}{ccccccc}
\hline \hline $\begin{array}{c}\text { Star ID } \\
\text { 2MASS }\end{array}$ & $\begin{array}{c}\Delta \mathrm{T}_{\text {eff }} \\
+100 \mathrm{~K}\end{array}$ & $\begin{array}{c}\Delta \log (\mathrm{g}) \\
+0.3 \mathrm{cgs}\end{array}$ & $\begin{array}{c}\Delta[\mathrm{M} / \mathrm{H}] \\
+0.3 \mathrm{dex}\end{array}$ & $\begin{array}{c}\Delta \mathrm{V}_{\mathrm{t}} \\
+0.3 \mathrm{~km} \mathrm{~s}^{-1}\end{array}$ & $\sigma / \sqrt{ }(\mathrm{N})$ & $\Delta$ Total \\
\hline $17285469-1627232$ & +0.09 & -0.02 & +0.05 & -0.19 & 0.05 & 0.22 \\
$17310360-1700146$ & +0.06 & -0.02 & +0.07 & -0.19 & 0.00 & 0.21 \\
$17295898-1652101$ & +0.07 & -0.02 & +0.06 & -0.20 & 0.05 & 0.23 \\
\hline
\end{tabular}


TABLE 6

$\alpha$-Element Abundance Summary

\begin{tabular}{ccccccc}
\hline \hline Field & $\begin{array}{c}{[\mathrm{O} / \mathrm{Fe}]} \\
\text { Median }\end{array}$ & $\sigma$ & $\begin{array}{c}{[\mathrm{Si} / \mathrm{Fe}]} \\
\text { Median }\end{array}$ & $\sigma$ & $\begin{array}{c}{[\mathrm{Ca} / \mathrm{Fe}]} \\
\text { Median }\end{array}$ & $\sigma$ \\
\hline \multicolumn{7}{c}{$[\mathrm{Fe} / \mathrm{H}]<-0.5$} \\
\hline$(-5.5,-7)$ & +0.61 & 0.14 & +0.31 & 0.07 & +0.30 & 0.11 \\
$(-4,-9)$ & +0.57 & 0.14 & +0.38 & 0.16 & +0.33 & 0.11 \\
$(+8.5,+9)$ & +0.59 & 0.11 & +0.38 & 0.09 & +0.27 & 0.10 \\
\hline \multicolumn{7}{c}{$[\mathrm{Fe} / \mathrm{H}]>-0.5$} \\
\hline$(-5.5,-7)$ & +0.14 & 0.18 & -0.03 & 0.13 & +0.09 & 0.12 \\
$(-4,-9)$ & +0.22 & 0.19 & +0.13 & 0.12 & +0.21 & 0.13 \\
$(+8.5,+9)$ & +0.27 & 0.22 & +0.02 & 0.11 & +0.05 & 0.14 \\
\hline
\end{tabular}

Note. - The $\sigma$ values listed in the table represent the scatter around a best fit line to either the $[\mathrm{Fe} / \mathrm{H}]<-0.5$ or $[\mathrm{Fe} / \mathrm{H}]>-0.5$ stars in each field. 